\title{
WAP SYSTEMS AND LABELED SUBSHIFTS
}

\author{
ETHAN AKIN AND ELI GLASNER
}

\section{Contents}

Introduction 2

1. WAP systems 6

1.1. Transitivity, Recurrence and Enveloping Semigroups 6

$\begin{array}{ll}\text { 1.2. The enveloping semigroup of WAP systems } & 12\end{array}$

1.3. The Birkhoff Center, CP and CT systems 15

1.4. Coalescence, LE, HAE and CT-WAP systems 21

1.5. Discrete suspensions and spin constructions 30

2. Labels and their dynamics 36

2.1. The space of labels 36

2.2. The action of $F I N(\mathbb{N})$ on $\mathcal{L} \mathcal{A B}$

3. Labeled subshifts 54

3.1. Integer expansions $\quad 54$

3.2. Labeled integers $\quad 58$

3.3. Subshifts 61

4. WAP labels and their subshifts $\quad 77$

4.1. Simple, semi-simple and finitary labels 77

4.2. WAP subshifts 84

4.3. Constructions and examples 87

5. Dynamical properties of $X(\mathcal{M}) \quad 97$

5.1. Translation finite subsets of $\mathbb{Z} \quad 97$

5.2. Non-null and non-tame labels 100

5.3. Gamow transformations 106

5.4. Ordinal constructions 108

6. Scrambled sets 116

$\begin{array}{lll}\text { Appendix A. Directed sets and nets } & 122\end{array}$

Appendix B. Ellis semigroups and Ellis actions 123

Appendix C. The Stone-Čech compactification 126

Date: August 18, 2016.

Key words and phrases. WAP, HAE, LE dynamical systems, space of labels, expanding functions, enveloping semigroup, adherence semigroup, subshifts, countable subshifts, symbolic dynamics, null, tame.

2010 Mathematical Subject Classification 37Bxx, 37B10, 54H20, 54H15. 
$\begin{array}{lr}\text { References } & 134\end{array}$

Index

\section{INTRODUCTION}

The main object of this work is to present a powerful method of construction of subshifts which we use chiefly to construct WAP systems with various properties. Among many other applications of these so called labeled subshifts, we obtain examples of null as well as non-null WAP subshifts, WAP subshifts of arbitrary countable (Birkhoff) height, and completely scrambled WAP systems of arbitrary countable height. We also construct LE but not HAE subshifts, and recurrent non-tame subshifts. Of course all of these notions, with some or all of which the reader may not be familiar, will be defined and illustrated in due course.

The notion of weakly almost periodic (WAP) functions on a locally compact abelian group $G$ was introduced by Eberlein [12], generalizing Bohr's notion of almost periodic (AP) functions. As the theory of AP functions was eventually reduced to the study of the largest topological group compactification of $G$, so the theory of WAP functions can be reduced to the study of the largest semitopological semigroup compactification of $G$. Following Eberlein's work there evolved a general theory of WAP functions on a general topological group $G$, or even more generally, on various type of semigroups. From the very beginning it was realized that a dual approach, via topological dynamics, is a very fruitful tool as well as an end in itself. Thus in the more recent literature on the subject one is usually concerned with WAP dynamical systems $(X, G)$. These are defined as continuous actions of the group $G$ on a compact Hausdorff space $X$ such that, for every $f \in C(X)$, the weak closure of the orbit $\{f \circ g: g \in G\}$ is weakly compact. The turning point toward this view point is the paper of Ellis and Nerurkar [14], which used the famous double limit criterion of Grothendieck to reformulate the definition of WAP dynamical systems as those $(X, G)$ whose enveloping semigroup $E(X, G)$ consists of continuous maps (and is thus a semi-topological semigroup).

In the last two decades the theory of WAP dynamical systems was put into the broader context of hereditarily almost equicontinuous (HAE) and tame dynamical systems. The starting point for this direction was the proof, in the work [5] of Akin Auslander and Berg, that WAP systems are HAE. For later development along these lines see e.g. [22].

Most of the extensive literature on the subject of WAP functions and WAP dynamical systems has a very abstract flavor. The research in these works is mostly concerned with related questions in harmonic analysis, Banach space theory, and the topology and the algebraic structure of the universal WAP 
semigroup compactification. Very few papers deal with presentations and constructions of concrete WAP dynamical systems. As a few exceptions let us point out the works of Katznelson-Weiss [32], Akin-Auslander-Berg [4], Downarowicz [11] and Glasner-Weiss [25, Example 1, page 349]. Even in these few works the attention is usually directed toward examples of recurrent WAP topologically transitive systems. These are the (usually metric) WAP dynamical systems which admit a recurrent transitive point.

A point $x$ in a metric dynamical system $(X, G)$ is a point of equicontinuity if for every $\epsilon>0$ there is a $\delta>0$ such that $d\left(g x, g x^{\prime}\right)<\epsilon$ for every $x^{\prime} \in B_{\delta}(x)$ and every $g \in G$. The system is called almost equicontinuous (AE) if it has a dense (necessarily $G_{\delta}$ ) subset of equicontinuity points. It is hereditarily almost equicontinuous (HAE) if every subsystem (i.e. non-empty closed invariant subset) is AE.

As our work deals almost exclusively with cascades (i.e. $\mathbb{Z}$-dynamical systems), in the sequel we will consider dynamical systems of the form $(X, T)$ where $T: X \rightarrow X$ is a homeomorphism. A large and important class of cascades is the class of symbolic systems or subshifts. We will deal only with subsystems of the Bernoulli dynamical system $\left(\{0,1\}^{\mathbb{Z}}, S\right)$, where $S$ is the shift transformation defined by

$$
(S x)_{n}=x_{n+1}\left(x \in\{0,1\}^{\mathbb{Z}}, n \in \mathbb{Z}\right) .
$$

We will call such dynamical systems subshifts. It was first observed in [22] that a subshift is HAE iff it is countable (see Proposition 1.23 below). In particular it follows that WAP subshifts are countable. Since a dynamical system which admits a recurrent non-periodic point is necessarily uncountable, it follows that in a WAP subshift the only recurrent points are the periodic points. These considerations immediately raise the question which countable subshifts are WAP, and how rich is this class? This question was the starting point of our investigation.

As we proceeded with our study of that problem we were able to construct several simple examples of both WAP and non WAP topologically transitive countable subshifts, but particular constructions of WAP subshifts turned out to be quite complicated. After many trials we finally discovered the beautiful world of labels. We begin with $F I N(\mathbb{N})$, the additive semigroup of nonnegative integer-valued functions with finite support defined on $\mathbb{N}$, the set of positive integers. A label is a subset $\mathcal{M}$ of $F I N(\mathbb{N})$ which is hereditary in the sense that $\mathbf{0} \leq \mathbf{m}_{1} \leq \mathbf{m}$ and $\mathbf{m} \in \mathcal{M}$ imply $\mathbf{m}_{1} \in \mathcal{M}$. The space $\mathcal{L} \mathcal{A B}$ of labels has a natural compact metric space structure.

For an odd positive integer $b=2 e+1$ every integer $t$ has a unique symmetric base $b$ expansion using the function $k=k_{b}$ defined by $k_{b}(i)=b^{i-1}$ for $i \in \mathbb{N}$. $t=\sum_{i=1}^{\infty} \epsilon_{i} k(i)$ with $\left|\epsilon_{i}\right| \leq e$ and with $\epsilon_{i}=0$ for all but finitely many indices $i$. We use $b \geq 5$ and extend $k$ to define $k: \mathbb{Z} \rightarrow \mathbb{Z}$ such that $k(-n)=-k(n)$ and 
$k(n+1)>3 \sum_{i=0}^{n} k(i)$ for $n \geq 0$. Together with $k$ we use an infinite partition $\left\{D_{\ell}: \ell \in \mathbb{N}\right\}$ of $\mathbb{N}$ by infinite subsets. The set $I P(k) \subset \mathbb{Z}^{1}$ consists of the sums of finite subsets of the image of $k$. That is, it is the set of $t$ such that $\epsilon_{i}= \pm 1$ whenever $\epsilon_{i} \neq 0$. Each $t \in I P(k)$ has a unique expansion $t=k\left(j_{1}\right)+\cdots+k\left(j_{r}\right)$ with $\left|j_{1}\right|>\cdots>\left|j_{r}\right|$. The length vector $\mathbf{r}(t) \in F I N(\mathbb{N})$ counts the number of occurrences of each member of the partition in the expansion. That is, $\mathbf{r}(t)_{\ell}=\#\left\{i: j_{i} \in D_{\ell}\right\}$. For a label $\mathcal{M}$ the set $A[\mathcal{M}]$ is the set of $t \in I P(k)$ such that $\mathbf{r}(t) \in \mathcal{M}$. For example, $\emptyset$ and $0=\{\mathbf{0}\}$ are labels with $A[\emptyset]=\emptyset$ and $A[0]=\{0\}$.

Once the base $b$ and the partition $\left\{D_{\ell}\right\}$ are fixed, there is a canonical injective, continuous map from the space of labels $\mathcal{L} \mathcal{A B}$ into $\{0,1\}^{\mathbb{Z}}, \mathcal{M} \mapsto x[\mathcal{M}]$ where $x[\mathcal{M}]$ is the characteristic function of the set $A[\mathcal{M}]$. Thus to a label $\mathcal{M}$ there is assigned a subshift $X(\mathcal{M})$, the orbit closure of $x[\mathcal{M}]$ under $S$.

We show in Theorem 3.7 that the set $I P(k)$ of all expanding times has upper Banach density zero. This, in turn, implies that for every label $\mathcal{M}$ the corresponding subshift $(X(\mathcal{M}), S)$ is uniquely ergodic with the point measure at $e=x[\emptyset]$ the unique invariant probability measure. It follows that each such system has zero topological entropy.

The space $\mathcal{L} \mathcal{A B}$ is naturally equipped with an action of the discrete semigroup $F I N(\mathbb{N})$,

$$
(\mathbf{r}, \mathcal{M}) \mapsto \mathcal{M}-\mathbf{r}=\{\mathbf{w} \in F I N(\mathbb{N}): \mathbf{w}+\mathbf{r} \in \mathcal{M}\}
$$

We denote the compact orbit closure of a label $\mathcal{M}$ under this action by $\Theta(\mathcal{M})$.

The key lemma which connects the two actions (the $F I N(\mathbb{N})$ action on labels and the shift $S$ on subshifts) is Lemma 3.20. Let $\left\{t^{i}\right\}$ be any sequence of in $I P(k)$ such that the sequence of smallest terms $\left\{\left|j_{r}\left(t^{i}\right)\right|\right\}$ tends to infinity and let $\left\{\mathbf{r}\left(t^{i}\right)\right\}$ be the corresponding sequence of length vectors. Then for any sequence of labels $\left\{\mathcal{N}^{i}\right\}$, the sequences $\left\{S^{t^{i}}\left(x\left[\mathcal{M}^{i}\right]\right)\right\}$ and $\left\{x\left[\mathcal{M}^{i}-\mathbf{r}\left(t^{i}\right)\right]\right\}$ are asymptotic in $\{0,1\}^{\mathbb{Z}}$.

We show that for a $F I N(\mathbb{N})$-recurrent label the corresponding $x[\mathcal{M}]$ is an $S$-recurrent point. At the other extreme we have the labels of finite type. For such a label $\mathcal{M}, e=x[\emptyset]$ is the only recurrent point in $X(\mathcal{M})$. These labels are particularly amenable to our analysis, which leads to a complete picture of the resulting subshift. In fact for a label $\mathcal{M}$ of finite type

$$
X(\mathcal{M})=\left\{S^{k} x[\mathcal{N}]: k \in \mathbb{Z}, \mathcal{N} \in \Theta(\mathcal{M})\right\}=\bigcup_{k \in \mathbb{Z}} S^{k} x[\Theta(\mathcal{M})]
$$

\footnotetext{
${ }^{1} I P(k)$ is an example of a symmetric IP set; for more information on IP sets and their connections to dynamical systems see e.g. [17], [15], [16], [27] and the recent paper [7].
} 
We show (see Corollary 3.35) that, given $\mathcal{M} \in \mathcal{L} \mathcal{A B}$, the maps $\Phi(\cdot)$, which for a closed $S$-invariant subset $Y$ of $X(\mathcal{M})$ is defined by

$$
Y \mapsto \Phi(Y)=\{\mathcal{N} \in \Theta(\mathcal{M}): x[\mathcal{N}] \in Y\}
$$

and $X(\cdot)$, which for a closed $F I N(\mathbb{N})$-invariant $\Psi \subset \Theta(\mathcal{M})$ is defined by

$$
\Psi \mapsto X(\Psi)=\text { the subshift generated by }\{x[\mathcal{N}]: \mathcal{N} \in \Psi\},
$$

have the following properties:

(1) The map $\Psi \mapsto X(\Psi)$ is one-to-one from the collection of closed $F I N(\mathbb{N})$ invariant subsets of $\Theta(\mathcal{M})$ into the collection of closed $S$-invariant subsets of $X(\mathcal{M})$.

(2) If $\mathcal{M}$ is of finite type then this map is surjective, i.e. every closed $S$-invariant subsets $Y$ of $X(\mathcal{M})$ is of the form $X(\Psi)$ for some closed $F I N(\mathbb{N})$ invariant $\Psi \subset \Theta(\mathcal{M})$, with

$$
\Phi(X(\Psi))=\Psi \text { and } Y=\Phi(X(\Psi)) .
$$

Thus, for a finite type label $\mathcal{M}$, the lattice of subsystems of the dynamical system $(\Theta(\mathcal{M}), F I N(\mathbb{N}))$ fully describes the lattice of subsystems of the dynamical system $(X(\mathcal{M}), S)$.

2 Two useful subcollections of the collection of finite type labels are the classes of the finitary labels and of the simple labels. For each label $\mathcal{M}$ in either one of these special classes, the corresponding subshift $X(\mathcal{M})$ is a countable WAP system whose enveloping semigroup structure is encoded in the structure of the label $\mathcal{M}$. This fact enables us to produce WAP subshifts with various dynamical properties by tinkering with their labels.

The recurrent labels are far less transparent and for these labels the image $x[\Theta(\mathcal{M})]$, which in this case is a Cantor set, forms only a meagre subset of the subshift $X(\mathcal{M})$. Nonetheless it seems that this image forms a kind of nucleus which encapsulates the dynamical properties of $X(\mathcal{M})$.

The table of contents will now give the reader a rough notion of the structure of our work. In the first section (section 1 ) we deal with abstract WAP systems and their enveloping semigroups and present simple examples of WAP and non-WAP systems. Among other considerations it is shown that topologically transitive WAP systems are coalescent and that a general WAP system is Ecoalescent. For an arbitrary separable metric system the hierarchies $z_{N W}$ and $z_{L I M}$ of non-wandering and $\alpha \cup \omega$ limiting procedures are studied. Both lead, by transfinite induction, to the Birkhoff center of the dynamical system. We call the ordinal at which the limiting $\alpha \cup \omega$ transfinite sequence stabilizes, the height of the system. In the final subsection we describe some general constructions like the discrete suspension, and the spin construction.

The space of labels is introduced and studied in section 2. The associated subshifts are introduced and studied in section 3. Section 4 deals with 
WAP labels and their corresponding subshifts. Finally, in sections 5 and 6 these tools are applied to obtain many interesting and subtle constructions of subshifts. Let us mention just a few. On the finite type side we obtain examples of null as well as non-null WAP subshifts, Example 5.23 (answering a question of Downarowicz); WAP subshifts of arbitrary (countable) height, Theorem 5.35; topologically transitive subshifts which are LE but not HAE, Example 3.39 and Remark 5.24 (these seem to be the first such examples); and completely scrambled WAP systems (although not subshifts) of arbitrary countable height, Example 6.13 (answering a question which is left open in Huang and Ye's work [31]). On the recurrent side we construct various examples of non-tame subshifts. Of course many questions are left open, especially when labels which are not of finite type are considered, and we present some of these throughout the work at the relevant places.

We thank Benjy Weiss for several helpful suggestions which greatly improved this work. We also thank the two anonymous referees for a careful reading of the manuscript and for their useful comments and corrections.

\section{WAP SYSTEMS}

\subsection{Transitivity, Recurrence and Enveloping Semigroups.}

The type of dynamical system of greatest interest for us is the cascade: a pair $(X, T)$ with $T$ is a homeomorphism on a nonempty compact Hausdorff space $X$, usually a metric space.

We follow some of the notation of [1] concerning relations on a space. In particular, we will use the the orbit relation

$$
O_{T}=\left\{\left(x, T^{n}(x)\right): x \in X, n \in \mathbb{Z}\right\}
$$

and the associated limit relation:

$$
R_{T}=\omega T \cup \alpha T \text {, }
$$

where

$$
\omega T=\left\{\left(x, x^{\prime}\right): x \in X, x^{\prime}=\lim _{i \rightarrow \infty} T^{n_{i}} x \text { with } n_{i} \nearrow \infty\right\}
$$

and

$$
\alpha T=\left\{\left(x, x^{\prime}\right): x \in X, x^{\prime}=\lim _{i \rightarrow \infty} T^{-n_{i}} x \text { with } n_{i} \nearrow \infty\right\} .
$$

$R_{T}$ is a pointwise closed relation (each $R_{T}(x)$ is closed) but not usually a closed relation (i.e. $R_{T}$ is usually not closed in $X \times X$ ).

We can regard the cascade $(X, T)$ as an action of the group $\mathbb{Z}$ on $X$ by $(t, x) \mapsto T^{t}(x)$. We will need certain results for more general actions. 
Let $\Gamma$ be a discrete, countable, commutative monoid ( = a semigroup with an identity element 1). Let $\Gamma^{\prime}=\Gamma \backslash\{1\}$.

A $\Gamma$-dynamical system is a pair $(X, \Gamma)$ where $X$ is a nonempty, compact Hausdorff space and $\Gamma$ acts on $X$ via a homomorphism of $\Gamma$ into the semigroup $C(X, X)$ of continuous maps from $X$ to itself, mapping 1 to the identity map, $i d_{X}$. We will write $\Gamma \cdot x=\{g x: g \in \Gamma\}$ for the $\Gamma$ orbit of a point $x \in X$.

We will call the action point transitive when it admits a transitive point, i.e. a point $x^{*}$ such that $\Gamma \cdot x^{*}$ is dense in $X .(X, \Gamma)$ is called minimal when every point of $X$ is a transitive point.

A subset $X_{0}$ is invariant if $x \in X_{0}$ implies $\Gamma \cdot x \subset X_{0}$. The closure of an invariant set is invariant since the action is continuous.

Given two sets $A, B \subset X$ we let $N(A, B)=\{g \in \Gamma: g A \cap B \neq \emptyset\}$. We call $x \in X$ a recurrent point if $x \in \overline{\Gamma^{\prime} \cdot x}$ or, equivalently, if $N(\{x\}, U) \cap \Gamma^{\prime} \neq \emptyset$ for every neighborhood $U$ of $x$. An open set $A$ (or more generally a set with nonempty interior) is called non-wandering if $N(A, A) \cap \Gamma^{\prime} \neq \emptyset$. If $\Gamma$ is a group then $A$ is called wandering if $\{g(A): g \in \Gamma\}$ is a pairwise disjoint collection indexed by $\Gamma$ and a set is either wandering or non-wandering. A point $x$ is non-wandering if every neighborhood $U$ of $x$ is non-wandering. It is easy to check that the set of non-wandering points is closed and contains the set of recurrent points. We will call the system central if every point is non-wandering or, equivalently, if $N(U, U) \cap \Gamma^{\prime} \neq \emptyset$ for for every nonempty open $U \subset X$.

We will call the system transitive if $N(U, V) \neq \emptyset$ for every pair of nonempty open $U, V \subset X$.

Proposition 1.1. Let $(X, \Gamma)$ be a $\Gamma$ dynamical system with $X$ metrizable.

(a) If the system is central then the set of recurrent points is a dense $G_{\delta}$ subset of $X$.

(b) If the system is transitive then the set of transitive points is a dense $G_{\delta}$ subset of $X$ and so the system is point transitive.

(c) If $\Gamma$ is a group and the system is point transitive, then it is transitive and the set of transitive points is invariant.

Proof: These are just easy versions of the results for cascades with $\Gamma=\mathbb{Z}$ and so we will just sketch the proofs. Let $\mathcal{B}$ be a countable base for $X$. For $A \subset X$ let $\left(\Gamma^{\prime}\right)^{-1}(A)=\bigcup_{g \in \Gamma^{\prime}}\left\{g^{-1}(A)\right\}$ with an analogous definition for $(\Gamma)^{-1}(A)=\left(\Gamma^{\prime}\right)^{-1}(A) \cup A$.

(a): Let $\mathcal{A}$ be a finite cover of $X$ by elements of $\mathcal{B}$.

$$
\text { Recur }=\bigcap_{\mathcal{A}} \bigcup_{U \in \mathcal{A}} U \cap\left(\Gamma^{\prime}\right)^{-1}(U)
$$

is the set of recurrent points and it is the countable intersection of dense open sets when $(X, \Gamma)$ is central. 
(b): The set of transitive points is $\bigcap_{U \in \mathcal{B}}(\Gamma)^{-1}(U)$ and this is the countable intersection of dense open sets when $(X, \Gamma)$ is transitive.

(c): If $x$ is a transitive point and $U, V \subset X$ are nonempty open sets then there exist $g_{1}, g_{2}$ such that $g_{1} x \in U, g_{2} x \in V$. So $g_{2} g_{1}^{-1} \in N(U, V)$ which is defined since $\Gamma$ is a group.

For any monoid action $y \in \Gamma \cdot x$ implies $\Gamma \cdot y \subset \Gamma \cdot x$ with equality when $\Gamma$ is a group. Hence, if $y$ is a transitive point then $x$ is and the converse is true when $\Gamma$ is a group.

If $X_{0} \subset X$ is nonempty, closed and invariant, then $\Gamma$ acts on $X_{0}$ by restriction, and we call $\left(X_{0}, \Gamma\right)$ a subsystem of $(X, \Gamma)$. In particular, the orbit closure $\overline{\Gamma \cdot x}$ is a closed, invariant set for any $x \in X$. By the compactness and the usual Zorn's Lemma argument, any nonempty, closed and invariant subset contains a nonempty, closed and invariant subset $M$ which is minimal with respect to inclusion. This is equivalent to the condition that the subsystem $(M, \Gamma)$ is minimal in the previously mentioned sense, i.e. every point $x \in M$ is a transitive point for $(M, \Gamma)$. Since the intersection of closed, invariant sets is closed and invariant, it follows that distinct minimal subsets are disjoint.

A not necessarily closed subset $X_{0}$ is orbit-closed if $x \in X_{0}$ implies $\overline{\Gamma \cdot x} \subset$ $X_{0}$. An orbit-closed set is invariant and a closed, invariant set is orbit-closed.

In particular, a cascade $(X, T)$ is transitive if for every two non-empty open sets $U, V$ in $X$ there is an $n \in \mathbb{Z}$ with $T^{-n} U \cap V \neq \emptyset$. By Proposition 1.1, if $X$ is metrizable, then transitivity is equivalent to point transitivity and implies that $X_{t r}$, the set of transitive points, is a dense $G_{\delta}$ subset of $X$.

A space is Polish if it is separable and admits a complete metric, e.g. a compact metric space. Since a Polish space is separable the set of isolated points is finite or countably infinite. A nonempty Polish space without isolated points is a union of Cantor subsets, see, e.g. [3, Proposition 2.3]. Since a $G_{\delta}$ subset of a Polish space is Polish, any nonempty $G_{\delta}$ subset $A$ of a Polish space either contains points isolated in $A$ or contains a Cantor Set. In particular, in a countable Polish space the isolated points are dense. Note that the set of rationals in $\mathbb{R}$ is not Polish.

The action of $\Gamma$ on $X$ is faithful if $g_{1} x=g_{2} x$ for all $x \in X$ implies $g_{1}=g_{2}$, i.e. the homomorphism from $\Gamma$ to $C(X, X)$ is injective. If $\breve{\Gamma}$ is the image of $\Gamma$ in $C(X, X)$ then $\breve{\Gamma}$ is a countable, abelian submonoid of $C(X, X)$ which acts faithfully on $X$. We call the action of $\Gamma$ on $X$ weakly faithful if $g x=x$ for all $x \in X$ implies $g=1$, i.e. the only element of $\Gamma$ which acts as the identity is 1 .

We let $\Gamma_{u}$ denote the group of units in $\Gamma$, i.e. the set of elements $g \in \Gamma$ such that there exists a -necessarily unique- inverse $\bar{g}$ such that $g \bar{g}=\bar{g} g=1$. 
Proposition 1.2. Let $(X, \Gamma)$ be a point transitive $\Gamma$ dynamical system. Assume that $X$ is not perfect, i.e. it contains isolated points.

(a) Assume that the action is weakly faithful. If some isolated point $x^{*}$ of $X$ is a transitive point, then the set of transitive points is $\Gamma_{u} x^{*}$ and these are all isolated points. None of the transitive points is recurrent.

(b) If $\Gamma$ is a group then the set of transitive points is the countable, dense open set of isolated points. Moreover, this set consists of a single $\Gamma$ orbit.

Proof: (a) If $x^{*}$ is an isolated transitive point and $\bar{x}$ is another transitive point then there exists $g^{*} \in \Gamma$ such that $g^{*} \bar{x}=x^{*}$ since the orbit of $\bar{x}$ meets the clopen set $\left\{x^{*}\right\} .\left(g^{*}\right)^{-1}\left(x^{*}\right)=\left\{y \in X: g^{*} y=x^{*}\right\}$ is a clopen set containing $\bar{x}$. Because $x^{*}$ is a transitive point, there exist $\bar{g} \in \Gamma$ such that $\bar{g} x^{*} \in\left(g^{*}\right)^{-1}\left(x^{*}\right)$ and so $g^{*} \bar{g}\left(x^{*}\right)=x^{*}$. Because $\Gamma$ is abelian, this implies that $g^{*} \bar{g}$ acts as the identity on $\Gamma x^{*}$ which is dense in $X$. Because the action is continuous and weakly faithful, it follows that $g^{*} \bar{g}=1$. Commutativity implies that $g^{*}$ and $\bar{g}$ are inverses in $\Gamma$ and so lie in $\Gamma_{u}$. Thus, $\bar{x} \in \Gamma_{u} x^{*}$.

On the other hand, if $g \in \Gamma_{u}$ and $x=g x^{*}$ then since $g$ is invertible, $x^{*} \in \Gamma x$ and so $\Gamma x^{*}$ is contained in - actually it equals $-\Gamma x$ and so the latter is dense. That is, $x \in X_{t r}$.

Since the elements of $\Gamma_{u}$ act as homeomorphisms and $x^{*}$ is isolated, it follows that every element of $\Gamma_{u} x^{*}$ is isolated.

Finally, if $g x^{*}=x^{*}$ then as above $g=1$. Hence, $\Gamma^{\prime} x^{*}$ is disjoint from the clopen set $\left\{x^{*}\right\}$. Hence, $x^{*}$ is not recurrent.

(b) By replacing $\Gamma$ by $\breve{\Gamma}$ we may assume that the action is faithful. Observe that the $\Gamma$ orbit is the same as the $\breve{\Gamma}$ orbit for every point of $X$. If $x^{*}$ is any isolated point and $\bar{x}$ is a transitive point then there exists $g^{*} \in \Gamma$ such that $g^{*} \bar{x}=x^{*}$. Because $\Gamma$ is a group, it acts via homeomorphisms and so $\bar{x}$ is an isolated point as well as a transitive point. Since $\Gamma$ is a group, $\Gamma_{u}=\Gamma$ and so applying (a) we obtain that the orbit $\Gamma \bar{x}$ is the set of transitive points all of which are isolated. There are countably many because $\Gamma$ is countable and the set is dense because $\bar{x}$ is a transitive point.

Corollary 1.3. If $(X, \Gamma)$ is a transitive $\Gamma$ dynamical system with $X$ countable and $\Gamma$ a group, then $X$ is metrizable and the set of transitive points is the dense open set of isolated points. Moreover, this set consists of a single $\Gamma$ orbit.

Proof: We first observe that a countable compact space has a countable base and so is metrizable. For each pair $(x, y)$ of distinct points of $X$, choose $U_{(x, y)}$ an open set containing $x$ and with $y \in X \backslash \overline{U_{(x, y)}}$. Since $X$ is countable $\left\{U_{(x, y)}\right\}$ is a countable collection of open sets. Using compactness it is easy to check that the finite intersections of such sets form a basis for $X$. Hence, $X$ 
is compact and metrizable. Because it is countable, no open subset contains a Cantor set and so the isolated points are dense. Furthermore, since the action is transitive, it is point transitive by Proposition 1.1 (b). The result then follows from Proposition 1.2 (b).

Example 1.4. Let $\Gamma$ be the one-point compactification of $\mathbb{N}$ with the multiplication $t \cdot s=\min (s, t)$. Thus, $\Gamma$ is a countable, compact, abelian topological monoid (i.e. the multiplication is jointly continuous). The point $\infty$ at infinity is the identity element and is the one non-isolated point. The action of $\Gamma$ on itself by multiplication is faithful and $\infty$ is the unique transitive point. Thus, the system is point transitive but not transitive. Contrast this with Proposition 1.1. The set $\{1\}$ is the unique minimal subset of $\Gamma$.

The system $(X, \Gamma)$ is called weakly mixing when the diagonal action of $\Gamma$ on $X \times X$ is transitive.

For a cascade $(X, T)$ we recall the definitions of $\epsilon$-chains and chain transitivity. Given $\epsilon>0$ an $\epsilon$-chain from $x$ to $y$ is a finite sequence $x=x_{0}, x_{1}, \ldots, x_{n}=$ $y$ such that $n>0$ and $d\left(T\left(x_{i}\right), x_{i+1}\right)<\epsilon$ for $i=0, \ldots, n-1$. The system $(X, T)$ is chain transitive if for any pair of points $x, y \in X$ and any $\epsilon>0$ there is an $\epsilon$-chain going from $x$ to $y$. An asymptotic chain is an infinite sequence $\left\{x_{i}: i \in \mathbb{Z}_{+}\right\}$or $\left\{x_{i}: i \in \mathbb{Z}\right\}$ such that $\lim _{|i| \rightarrow \infty} d\left(T\left(x_{i}\right), x_{i+1}\right)=0$. It is a dense asymptotic chain if for every $N \in \mathbb{N}\left\{x_{i}: i \geq N\right\}$ is dense in $X$ (and in the $\mathbb{Z}$ case $\left\{x_{i}: i \leq-N\right\}$ is dense in $X$ as well). If $(X, T)$ is chain transitive and $x \in X$ then there exists a dense asymptotic chain $\left\{x_{i}: i \in \mathbb{Z}\right\}$ with $x=x_{0}$.

The following construction is due to Takens (see, e. g. [1, Chapter 4, Exercise 29] ).

Example 1.5. If $(X, T)$ is a chain transitive metric system and $\left\{x^{i}: i \in \mathbb{Z}\right\}$ is a dense asymptotic chain then let

$$
z^{i}= \begin{cases}\left(x^{i},(2 i+1)^{-1}\right) & \text { for } i \geq 0, \\ \left(x^{i},(2|i|)^{-1}\right) & \text { for } i<0 .\end{cases}
$$

Let $X^{*}=X \times\{0\} \cup\left\{z^{i}: i \in \mathbb{Z}\right\}, x^{*}=z^{0}$. Extend $T=T \times i d_{0}$ on $X \times\{0\}$ identified with $X$, by $T\left(z^{i}\right)=z^{i+1}$ for $i \in \mathbb{Z}$. Then $\left(X^{*}, T\right)$ is a topologically transitive metrizable system with transitive point $x^{*}$ and $X^{*}=X \cup O_{T}\left(x^{*}\right)$ and $X=\omega T\left(x^{*}\right)$. 
Now we return to $\Gamma$ actions.

The enveloping semigroup $E=E(X, \Gamma)$ of the dynamical system $(X, \Gamma)$ is defined as the closure in $X^{X}$ (with its compact, usually non-metrizable, pointwise convergence topology) of the image of $\Gamma$ in $C(X, X)$ considered as a subset of $X^{X}$. Since $\Gamma$ is a monoid, $E(X, \Gamma)$ is a monoid with the identity $i d_{X}$.

It follows directly from the definitions that, under composition of maps, $E$ forms a compact semigroup in which the operations

$$
p \mapsto p q \quad \text { and } \quad p \mapsto g p
$$

for $p, q \in E, g \in \Gamma$, are continuous. Since we have assumed that $\Gamma$ is commutative, it follows from continuity of the $\Gamma$ action that each $g \in \Gamma$ commutes with every $p \in E(X, \Gamma)$.

Notice that $\Gamma$ acts on $E$ by multiplication, so that $(E, \Gamma)$ is a $\Gamma$-system (though usually non-metrizable).

The elements of $E$ may behave very badly as maps of $X$ into itself; usually they are not even Borel measurable. However our main interest in $E$ lies in its algebraic structure and its dynamical significance. A key lemma in the study of this algebraic structure is the following (see Lemma B.1 in Appendix B):

Lemma 1.6 (Ellis-Numakura). Let $L$ be a compact Hausdorff semigroup in which all maps $p \mapsto p q$ are continuous. Then $L$ contains an idempotent ; i.e., an element $v$ with $v^{2}=v$.

Given two $\Gamma$ dynamical systems, say $(X, \Gamma)$ and $(Y, \Gamma)$, a continuous map $\pi: X \rightarrow Y$ is a homomorphism or an action map if it intertwines the $\Gamma$ actions, i.e. $\gamma \pi(x)=\pi(\gamma x)$ for every $x \in X$ and $\gamma \in \Gamma$. If $\pi$ is injective it expresses $X$ as a subsystem of $Y$. If $\pi$ is surjective it expresses $Y$ as a factor of $X$.

If $X_{0} \subset X$ is closed and invariant, then the inclusion of the subsystem $\left(X_{0}, \Gamma\right)$ into $X$ is an injective action map. Furthermore, $X_{0}$ is invariant with respect to the $E(X, \Gamma)$ action.

An injective action map induces, by restriction, a surjective, continuous monoid homomorphism (and an action map) $\pi^{*}: E(Y, \Gamma) \rightarrow E(X, \Gamma)$. A surjective action map $\pi: X \rightarrow Y$ induces a surjective, continuous monoid homomorphism (and an action map) $\pi_{*}: E(X, \Gamma) \rightarrow E(Y, \Gamma)$. Observe that if $\pi\left(x_{1}\right)=\pi\left(x_{2}\right)$ then for any $p \in E(X, \Gamma), \pi\left(p x_{1}\right)=p \pi\left(x_{1}\right)=p \pi\left(x_{2}\right)=\pi\left(p x_{2}\right)$.

The map $E(X, \Gamma) \times X \rightarrow X$ given by $(p, x) \mapsto p x$ is an action although the map is usually not jointly continuous. However, if $x \in X$ then the evaluation map $e v_{x}: E(X, \Gamma) \rightarrow X$ given by $p \mapsto p x$ is continuous and is an action map. A point $x^{*} \in X$ is a transitive point iff $e v_{x^{*}}$ is surjective and so yields $X$ as a factor of $E$.

For more details see e.g. [18, Chapter 1, Section 4] and [6]. 
Lemma 1.7. If $p \in E(X, \Gamma)$ is continuous at $x \in X$, then for any $q \in E(X, \Gamma)$ and $y \in X$ if $q y=x$ then $p q y=q p y$.

Proof: If $\left\{g^{i}\right\}$ is a net in $\Gamma$ which converges to $q$ pointwise then $\left\{g^{i} y\right\} \rightarrow$ $q y=x$ and so by continuity of $p$ at $x, p g^{i} y \rightarrow p q y$. But $p g^{i} y=g^{i} p y \rightarrow q p y$ since the elements of $\Gamma$ commute with $p$.

Proposition 1.8. If $x^{*}$ is a transitive point for $(X, \Gamma)$ and $p \in E(X, \Gamma)$ then the following are equivalent.

(i) $p$ is continuous on $X$.

(ii) For all $q \in E(X, \Gamma) p q=q p$ on $X$.

(iii) For all $q \in E(X, \Gamma) p q x^{*}=q p x^{*}$.

Proof: (i) $\Rightarrow$ (ii): This follows from Lemma 1.7 .

(ii) $\Rightarrow$ (iii): Obvious.

(iii) $\Rightarrow$ (i): Suppose the net $\left\{x^{i}\right\}$ in $X$ converges to $x$. To show that then $p x_{i} \rightarrow p x$, it suffices to show that every convergent subnet has limit $p x$. So we can assume that $\lim p x_{i}$ exists. Since $x^{*}$ is a transitive point, $e v_{x^{*}}$ is surjective and there are $q^{i} \in E(X, T)$ with $x^{i}=q^{i} x^{*}$. Let $\left\{q^{i^{\prime}}\right\}$ be a convergent subnet in $E(X, \Gamma)$ with limit $q$. Then, by continuity of $e v_{x^{*}}, q x^{*}=x$. By assumption (iii) $\lim p x^{i}=\lim p x^{i^{\prime}}=\lim p q^{i^{\prime}} x^{*}=\lim q^{i^{\prime}} p x^{*}=q p x^{*}=p q x^{*}=p x$.

\subsection{The enveloping semigroup of WAP systems.}

A dynamical system $(X, \Gamma)$ is called WAP (weakly almost periodic) when the elements of $E(X, \Gamma)$ are all continuous functions on $X$. Clearly for a WAP system $(X, \Gamma)$ the multiplication on its enveloping semigroup is continuous in each variable separately; i.e. $E(X, \Gamma)$ is an abelian semi-topological semigroup. The converse however is not necessarily true; see Proposition 1.13.

N.B. It still need not be true that the action $E(X, \Gamma) \times X \rightarrow X$ is jointly continuous, even in the case of a WAP cascade.

Corollary 1.9. If $(X, \Gamma)$ is a point transitive system with a transitive point $x^{*}$ then the following are equivalent.

(i) $(X, T)$ is WAP

(ii) $E(X, T)$ is abelian.

(iii) For every $p, q \in E(X, T) p q x^{*}=q p x^{*}$.

When these conditions hold $e v_{x^{*}}:(E(X, \Gamma), \Gamma) \rightarrow(X, \Gamma)$ is an isomorphism and there is a unique minimal subset of $X$. 
Proof: The equivalence of (i), (ii) and (iii) follows from Proposition 1.8

If $(X, \Gamma)$ is WAP and $p x^{*}=q x^{*}$ then $p=q$ on $\Gamma \cdot x^{*}$ because the elements of $\Gamma$ commute with $p$ and $q$. Since $\Gamma \cdot x^{*}$ is dense, $p=q$ by continuity. That is, $e v_{x^{*}}$ is injective and so is an isomorphism.

If $x_{i} \in M_{i}$ for minimal sets $M_{1}, M_{2}$ there exist $p_{1}, p_{2} \in E(X, \Gamma)$ s.t. $p_{i}\left(x^{*}\right)=$ $x_{i}$ and so $p_{2}\left(x_{1}\right)=p_{2}\left(p_{1}\left(x^{*}\right)\right) \in M_{1}$ while $p_{1}\left(x_{2}\right)=p_{1}\left(p_{2}\left(x^{*}\right)\right) \in M_{2}$. Since $E(X, T)$ is commutative, $M_{1} \cap M_{2} \neq \emptyset$ and so $M_{1}=M_{2}$.

Proposition 1.10. If $e v_{x^{*}}: E(X, \Gamma) \rightarrow X$ is an homeomorphism (e.g. if $(X, \Gamma)$ is WAP with transitive point $\left.x^{*}\right)$ and $\left\{q^{i}\right\}$ is a net in $E(X, \Gamma)$ such that $\left\{q^{i} x^{*}\right\}$ converge to a point $x \in X$ then $\left\{q^{i}(z)\right\}$ converges in $X$ for every $z \in X$. In fact, $\left\{q^{i}\right\}$ converges pointwise to the unique $p \in E(X, \Gamma)$ such that $p\left(x^{*}\right)=x$. Thus, if $p \in E(X, T)$ and $\left\{q^{i}\left(x^{*}\right)\right\}$ converges to $p\left(x^{*}\right)$ in $X$ then $\left\{q_{i}\right\} \rightarrow p$ in $E(X, \Gamma)$.

Proof: Obvious by inverting the homeomorphism $e v_{x^{*}}$.

A dynamical system $(X, \Gamma)$ is called $A P$ (almost periodic) or equicontinuous if $\Gamma$ acts equicontinuously on $X$. Clearly an AP system is WAP and its enveloping semigroup is a (commutative) compact topological group. Note that there are (non-transitive) systems $(X, \Gamma)$ with $E(X, \Gamma)$ a (commutative) compact topological group which are not equicontinuous. This is the case e.g. in Example 1.12(a) below (see also [23, Example 6.5]). However, for a transitive system, $E(X, \Gamma)$ is a commutative compact group iff $(X, \Gamma)$ is equicontinuous.

Even in the cascade case, $e v_{x^{*}}$ a homeomorphism need not imply WAP.

Example 1.11. Let $X$ be a compact, connected metric space and $T=i d_{X}$, the identity map. So $E(X, T)=\left\{i d_{X}\right\}$. Let $\left\{x_{i}: i \in \mathbb{Z}\right\}$ be a sequence of distinct points in $X$ such that $\lim _{|i| \rightarrow \infty} d\left(x_{i}, x_{i+1}\right)=0$ and so that the positive and negative tails are dense in $X$. Thus, $\left\{x_{i}\right\}$ is a dense asymptotic chain for $(X, T)$. Following Example 1.5 we embed $(X, T)$ as a subsystem of $\left(X^{*}, T\right)$ with $X^{*}=X \cup O_{T}\left(x^{*}\right)$ and $\left\{T^{i} x^{*}\right\}$ asymptotic to $\left\{x_{i}\right\}$.

Now assume that $y \in X$ and $T^{n_{k}} x^{*}$ converges to $y$ ( and so with $\left|n_{k}\right| \rightarrow \infty$ ). Then $T^{n_{k}+N} x^{*}$ converges to $T^{N} y=y$. Furthermore, for any $z \in X_{0}, T^{n_{k}} z=z$ converges to $z$. Thus, $T^{n_{k}}$ converges pointwise to the function which is the identity on $X$ and which is constantly $y$ on the orbit of $x^{*}$. In particular, $e v_{x^{*}}$ : $\left(E\left(X^{*}, T\right), T\right) \rightarrow\left(X^{*}, T\right)$ is an isomorphism. On the other hand, $E\left(X^{*}, T\right)$ is not commutative and none of the elements of $E\left(X^{*}, T\right)$ are continuous except for the iterates $T^{n}$. 
Example 1.12. An abelian enveloping semigroup does not imply WAP in general.

(a) Let the circle be $\mathbb{R} / \mathbb{Z}$. Let $X=\mathbb{R} / \mathbb{Z} \times \mathbb{Z}^{*}$ where $\mathbb{Z}^{*}$ is the one-point compactification of $Z$. Define $T$ to be the identity on $\mathbb{R} / \mathbb{Z} \times\{\infty\}$ and by $(t, n) \mapsto\left(t+3^{-(|n|+1)}, n\right)$. On each circle the map is just a rotation, hence equicontinuous, and so is WAP. Therefore the enveloping semigroup is commutative. Consider the sequence $\left\{T^{\Sigma_{i=0}^{k} 3^{i}}\right\}$. On $\mathbb{R} / \mathbb{Z} \times\{n\}$ this is eventually constant at the rotation $t \mapsto t+\sum_{i=0}^{|n|} 3^{-(|n|-i+1)}$. As $|n| \rightarrow \infty$ this approaches the rotation $t \mapsto t+\frac{1}{2}$. But on the circle $\mathbb{R} / \mathbb{Z} \times\{\infty\}$ every element of the enveloping semigroup restricts to the identity.

(b) A countable example is the spin $(Z, T)$ of the identity on $\mathbb{Z}^{*}$. $Z$ is a subset of $\mathbb{Z}^{*} \times \mathbb{Z}^{*}$

$$
\begin{gathered}
Z=\left(\mathbb{Z}^{*} \times\{\infty\}\right) \cup \bigcup_{n \in \mathbb{Z}}\{[-|n|,+|n|] \times\{n\}\}, \\
T(x)= \begin{cases}(\infty, \infty) & \text { for } x=(\infty, \infty) \\
(t+1, \infty) & \text { for } x=(t, \infty) \\
(t+1, n) & \text { for } x=(t, n) \text { with }-n \leq t<n, \\
(-n, n) & \text { for } x=(n, n) .\end{cases}
\end{gathered}
$$

The following proposition describes the equivalences for what might be called local WAP.

Proposition 1.13. For a system $(X, \Gamma)$ the following are equivalent.

(i) Multiplication for the enveloping semigroup is continuous in each variable; i.e. $E(X, \Gamma)$ is a semi-topological semigroup.

(ii) Every element of the enveloping semigroup has a continuous restriction on the orbit closure of each element of $X$.

(iii) The enveloping semigroup is commutative.

(iv) Each orbit closure in $X$ is a WAP system.

Proof: Since each orbit closure is invariant for the enveloping semigroup and since the topology of the latter is pointwise convergence, each of these conditions holds for $(X, \Gamma)$ iff it holds for the restriction to each orbit closure. This restricts to the topologically transitive case for which (iii) implies (ii) by Proposition 1.8. Because of pointwise convergence, (ii) implies (i) is obvious. If $p, q$ are in the enveloping semigroup and $g^{j}$ is a net converging to $q$ then $p g^{j}=g^{j} p$ and the separate continuity of multiplication at $\mathrm{p}$ implies $p q=q p$. 
In the case of a cascade $(X, T)$, we write $(E(X, T), A(X, T))$ for the enveloping semigroup and the ideal which is the adherence semigroup (= the limit points of $\left\{T^{n}\right\}$ as $n \rightarrow \pm \infty$ ) (see Definition C.9 in Appendix C). Let $T_{*}$ on $E(X, T)$ be the homeomorphism given by $T_{*}(p)=T p=p T$. Thus, $y \in R_{T}(x)$ iff $y=p x$ for some $p \in A(X, T)$ and $A(X, T)=R_{T_{*}}\left(i d_{X}\right)$. Note that $\left(E(X, T), T_{*}\right)$ is a - usually non-metrizable - cascade.

\subsection{The Birkhoff Center, CP and CT systems.}

We now restrict our attention to cascades.

A point $x \in X$ was defined above to be recurrent when it is in the closure of $\left\{T^{n}(x): n \neq 0\right\}$. It follows that $x$ is recurrent iff $x \in R_{T}(x)$ and so iff there exists $p \in A(X, T)$ such that $p x=x$. That is, $I s o_{x}=\{p \in E(X, T): p x=x\}$ intersects $A(X, T)$ in a nonempty, closed subsemigroup. Hence, there exists an idempotent $u \in A(X, T)$ such that $u x=x$. On the other hand, $x$ is nonrecurrent iff the orbit $\mathcal{O}_{T}(x)$ is disjoint from the limit set $R_{T}(x)$. The closure of the set of recurrent points is the Birkhoff Center. We will denote by Cent $_{T}$ the Birkhoff center for $(X, T)$.

A open set $A \subset X$ was defined to be wandering if $\left\{T^{n}(A): n \in \mathbb{Z}\right\}$ is a bi-infinite sequence of pairwise disjoint sets. Otherwise, there exists $n>0$ such that $A \cap T^{n}(A) \neq \emptyset$ and $A$ is called non-wandering. A point $x$ is nonwandering if every open neighborhood of $x$ is non-wandering. A recurrent point is clearly non-wandering. Recall that $(X, T)$ is called central if every point is non-wandering. In that case, with $X$ metrizable, the set of recurrent points forms a dense $G_{\delta}$ subset, by Proposition 1.1. Even in the non-metrizable case, Ellis proved that the set of recurrent points is dense if $(X, T)$ is central, see, e.g. [2, Proposition 5.18].

Let $(X, T)$ be a cascade with $X$ metrizable and hence separable. Define $z_{C A N}(X)$ to be the complement of the set of isolated points in $X$. Let $z_{N W}(X)$ be the complement of the union of all wandering open sets, i.e. the set of nonwandering points. Note that if a point is isolated and non-periodic then it is wandering. Thus, if there are no isolated periodic points, then $z_{N W}(X) \subset$ $z_{C A N}(X)$. Since $T$ is a homeomorphism, the set of isolated points is invariant. Hence, both $z_{N W}(X)$ and $z_{C A N}(X)$ are closed invariant sets.

Let $z_{L I M}(X)=\overline{R_{T}(X)}$. If an open set $U$ meets $\overline{R_{T}(X)}$ then there exist $x, y \in X$ such that $y \in U \cap R_{T}(x)$ and so for infinitely many $n \in \mathbb{Z}, T^{n}(x) \in U$. In particular, $U$ is non-wandering. It follows that $z_{L I M}(X) \subset z_{N W}(X)$. 
For each of these operators $z$ we define the descending transfinite sequence of closed sets by

$$
z_{0}(X)=X, \quad z_{\alpha+1}(X)=z\left(z_{\alpha}(X)\right), \quad z_{\beta}(X)=\bigcap_{\alpha<\beta} z_{\alpha}(X),
$$

for $\beta$ a limit ordinal.

We say that the sequence stabilizes at $\beta$ when $z_{\beta}(X)=z_{\beta+1}(X)$ in which case it is constant from then on. The first $\beta$ at which stabilization occurs for the $C A N / N W / L I M$ sequence is called the $C A N / N W / L I M$ level. Since $X$ is a separable metric space, each level is a countable ordinal (because for any limit ordinal $\beta$ less than or equal to the level $\left\{X \backslash z_{\alpha}(X): \alpha<\beta\right\}$ is a strictly increasing open cover of the Lindelöf space $X \backslash z_{\beta}(X)$, and so there is a sequence of ordinals $\left\{\alpha_{n}<\beta\right\}$ with limit $\beta$. Hence, $\beta$ is smaller than the first uncountable ordinal and so the level is a countable ordinal).

We let $z_{\infty}(X)=z_{\beta}(X)$ when the sequence stabilizes at $\beta$. Clearly $z_{L I M, \alpha}(X)$ $\subset z_{N W, \alpha}(X)$ for all $\alpha$. Recall that when $(X, T)$ is non-wandering, i.e. $z_{N W}(X)$ $=X$, then the recurrent points are dense. Since all recurrent points are contained in $z_{L I M, \infty}(X)$ it follows that $z_{L I M, \infty}(X)=z_{N W, \infty}(X)$ is the closure of the set of recurrent points, i.e. the Birkhoff Center. While the two transfinite sequences are eventually equal, they can stabilize at different levels.

At each stage of the $z_{C A N}$ sequence, the set of isolated points is countable. Hence, $X \backslash z_{C A N, \infty}(X)$ is a countable union of countable sets and so is itself countable. On the other hand, $z_{C A N, \infty}(X)$ has no isolated points. So every non-empty subset which is open in $z_{C A N, \infty}$ contains a Cantor set. Thus, $X$ $\backslash z_{C A N, \infty}$ is the maximum countable open subset of $X$ and

$$
z_{C A N, \infty}=\{x \in X \text { : every neighborhood of } x \text { is uncountable }\} .
$$

In particular, if $X$ is compact and countable then $z_{C A N, \infty}=\emptyset$. Since the intersection of the decreasing family of nonempty closed sets has a nonempty intersection, $\beta_{C A N}(X)$ is not a limit ordinal if $X$ is compact and countable.

Definition 1.14. We will call the ordinal $\beta_{L I M}(X)$ at which the $z_{L I M}$ sequence stabilizes, the height of $(X, T)$.

Call $(X, T)$ semi-trivial (hereafter ST) if $R_{T}=X \times\{e\}$ for a point, a fixed point, $e \in X$. That is, for every $x \in X, R_{T}(x)=\{e\}$. Call $(X, T)$ center periodic (hereafter $\mathrm{CP}$ ) if the only recurrent points are periodic. Call $(X, T)$ center trivial (hereafter $\mathrm{CT}$ ) if there is a unique recurrent point $e$, necessarily a fixed point, and so the Birkhoff center is $\{e\}$. Call $(X, T)$ min center trivial (hereafter minCT) if there is a unique minimal point $e$, necessarily a fixed point.

Clearly, ST implies CT and CT implies CP and minCT. A nontrivial system is $\mathrm{ST}$ iff it is a CT system of height 1. For a minCT system we will denote by 
$u$ the retraction to the fixed point $e$. In general, if $p \in E(X, T)$ is minimal, then $p x$ is a minimal point of $X$ for all $x \in X$. So if $(X, T)$ is minCT, then $u$ is the only minimal element of $E(X, T)$. Thus, $\left(E(X, T), T_{*}\right)$ is minCT.

For any cascade $(X, T)$, if a point $x \in X$ is periodic, then its orbit closure is finite. If $x$ is non-recurrent then its orbit is disjoint from $R_{T}(x)$. In exactly these cases, $x$ is isolated in its orbit closure. In particular, an isolated point is recurrent iff it is periodic. It follows that a cascade is CP iff every point is isolated in its orbit closure.

If $(X, T)$ is central, then the recurrent points are dense and so every isolated point is recurrent and so is periodic.

If a metrizable $(X, T)$ is $\mathrm{CP}$ and central then the recurrent points - which are all periodic - form a dense $G_{\delta}$ and so by the Baire Category Theorem, $\left\{x: T^{n}(x)=x\right\}$ has nonempty interior for some positive $n$. In fact, the union of such interiors is dense in $X$. If there are only countably many periodic points then this open dense set is countable and Polish and so the isolated points are dense in $X$. Also, every isolated point is periodic because $X$ is central

The identity on any compact space defines a CP system and the finite product of CP's is CP (not the infinite product since the product of periodic orbits can contain an adding machine). Any subsystem or factor of a CP system is $\mathrm{CP}$ (since any recurrent point in the factor lifts to some recurrent point in the top). Inverse limit does not work. Again, an adding machine is the inverse limit of periodic orbits.

Remark 1.15. A nontrivial CP system $(X, T)$ can never be weak mixing, i.e. $(X \times X, T \times T)$ is never topologically transitive. If $x^{*}$ is a transitive point for a CP system $(X, T)$ then it is isolated in $X$. If $x^{*}$ is an isolated, transitive point for a nontrivial system $(X, T)$ then $T\left(x^{*}\right) \neq x^{*}$ and so $U=\left\{x^{*}\right\}$ and $V=\left\{T\left(x^{*}\right)\right\}$ are nonempty open subsets of $X$, but $N(U \times U, U \times V)=\emptyset$.

The CT condition is closed under arbitrary products and subsystems. In particular, the enveloping semigroup of a CT system is $\mathrm{CT}$. The retraction $u$ to the fixed point $e \in X$ is the unique fixed point in $E(X, T)$ (the system is minCT). Also, it is the unique idempotent in $A(X, T)$. If $\pi:(X, T) \rightarrow(Y, S)$ is an action map and $X$ is CT then $Y$ is. In general, $(X, T)$ is CT iff $(Y, S)$ is CT and $\pi^{-1}(e)$ is a CT subsystem of $X$.

Mapping $(X, T)$ to the factor system on $X /$ Cent $_{T}$, where Cent $_{T}$ is collapsed to a single point, defines a functor from compact systems to CT systems. An action map $X \rightarrow Y$ with $Y$ CT factors through the projection from $X$ to $X /$ Cent $_{T}$ and so the functor is adjoint to the inclusion functor. 
If $(X, T)$ is a countable $\mathrm{CT}$ system, then $e$ is not an isolated point in any invariant closed subset of $X$ except $\{e\}$ itself. Thus, if the Cantor sequence stabilizes at $\beta+1$ then $z_{C A N, \beta}=\{e\}$ and conversely. In that case, for any $\alpha \leq \beta z_{L I M, \alpha} \subset z_{N W, \alpha} \subset z_{C A N, \alpha}$. That is, up to $\alpha=\beta$ the isolated points are all wandering.

A CT system $(X, T)$ has height 0 iff $X=\{e\}$, i.e. the system is trivial, and has height 1 iff it is ST.

Proposition 1.16. (a) If $(X, T)$ is an ST system then it is WAP.

(b) If $(X, T)$ is a CT system with height at most 2 then $E(X, T)$ is commutative. If, in addition, $(X, T)$ is topologically transitive then it is WAP.

Proof: (a) If $(X, T)$ is ST, then $E(X, T)=\left\{T^{n}: n \in \mathbb{Z}\right\} \cup\{u\}$ where $u$ is the retraction onto $e$. So every element of $E(X, T)$ is continuous.

(b) If $p, q \in A(X, T)$ then $p q=u=q p$. Hence, the semigroup is abelian. So if $(X, T)$ is topologically transitive, then it is WAP by Proposition 1.8.

Let $S$ denote the shift homeomorphism on $\{0,1\}^{\mathbb{Z}}$, i.e. $S(x)_{i}=x_{i+1}$. We will be focusing much of our attention on nonempty subsystems of the full shift $\left(\{0,1\}^{\mathbb{Z}}, S\right)$. These are called subshifts.

Example 1.17. In his work [38] Shapovalov shows that within the class of countable subshifts one can find, for any countable ordinal $\alpha$, a subshift $X_{\alpha} \subset$ $\{0,1\}^{\mathbb{Z}}$ whose Birkhoff degree, i.e. its NW level, is $\alpha+1$. Now it is easy to verify that all of these subshifts $X_{\alpha}$ constructed by Shapovalov are in fact ST and therefore also WAP. One can make them topologically transitive by attaching a single orbit. Thus we conclude that the class of WAP, topologically transitive subshifts is rich enough to present every countable Birkhoff degree. Note however that being semi-trivial Shapovalov's original examples all are of height 2 and they become of height 3 when an orbit is attached to make them topologically transitive. As we will show later (Theorem 5.35) the class of WAP, topologically transitive subshifts is also rich enough to present every countable height.

Example 1.18. Various non-WAP examples.

Let $e=\overline{0}, x[0]=0^{\infty} i 0^{\infty}$, i.e. $x[0]_{0}=1$ and $x[0]_{i}=0$ otherwise. Here $\bar{\alpha}$ for a finite binary word $\alpha$ is the periodic orbit $\cdots \alpha \alpha \alpha \cdots \in\{0,1\}^{\mathbb{Z}}$. Thus $\overline{0}$ is the constant sequence $(\ldots, 0,0,0, \ldots)$. Let $X(0)$ be the ST subshift generated by $x[0]$. Thus, $(X(0), S)$ is isomorphic to translation on the one point compactification $\mathbb{Z}^{*}$ of $\mathbb{Z}$.

(a) For $k=1,2, \ldots$, let $b_{j}^{k}=1$ for $j=10^{n k}, n \in \mathbb{Z}$ and $=0$ otherwise. Let $(X, S)$ be the generated subshift. $R_{S}(X)=X(0)$ and so $(X, S)$ has height 2. We have $b^{k} \rightarrow x[0]$ as $k \rightarrow \infty$. The sequence $S^{10^{n !}} \rightarrow p$ in $A(X, T)$ with 
$p\left(b^{k}\right)=x[0]$ for all $k$ and $p(x[0])=e$. So $p$ is not continuous at $x[0]$, despite the fact that all of the points of $X \backslash X(0)$ are isolated. That is, the assumption of topological transitivity in Proposition 1.16 (b) is necessary.

(b) A topologically transitive system of height 1 with minimal set not a fixed point need not be WAP. Let $c$ be given by $c_{i}=1$ for $i=2 n,-1-2 n$ for $n \in \mathbb{N}$ and $=0$ otherwise, i.e. $c=(01)^{\infty}(10)^{\infty}$. The orbit closure of $c$ consists of $O_{S}(c)$ together with the periodic orbit $\{\overline{01}, \overline{10}\} . S^{-2 k}(c) \rightarrow \overline{01}, S^{2 k}(c) \rightarrow \overline{10}$. $S^{-2 k} \rightarrow p$ and $S^{2 k} \rightarrow q$ both $p, q$ are identity on the periodic orbit. Hence, $q=p q \neq q p=p$ on $c$.

(c) For $(Y, S)$ any compact metric system, let $(X, T)$ be the one point compactification of $(Y \times \mathbb{Z}, S \times t)$ with $t$ the translation on $\mathbb{Z}$. This is an ST system. If $S=i d_{Y}$ it is easy to build a countable sequence of periodic orbits with limit set $(X, T)$. The expanded system is CP with an uncountable center although there are only countably many periodic orbits.

(d) Call $x \in\{0,1\}^{\mathbb{Z}}$ selective if for any $n \geq 0$ the word $10^{n} 1$ occurs at most once in $x$. Let $X$ be the set of all selective $x$. Clearly, if $x$ is selective then $R_{S}(x) \subset X(0)$. Note that if $A \subset \mathbb{Z}$ is such that all the nonzero differences $a_{i}-a_{j}$ are distinct, then the characteristic function $\chi(A)$ of $A$ in $\{0,1,\}^{\mathbb{Z}}$, is a selective element. $(X, S)$ is an uncountable CT subshift with height 2 . Hence every orbit closure of a point in $X$ is WAP. It is not hard to see that $X$ itself is not WAP. Let $x \in X$ such that $x_{n_{k}}=1$ with $n_{k} \rightarrow \infty$. Let $z_{i}^{N}$ agree with $x_{i}$ for $|i| \leq N$ and equal 0 otherwise. Then $\left\{z^{N}\right\}$ is a sequence in $X$ which converges to $x$. If a subnet of $T^{n_{k}}$ converges to $p \in E(X, S)$ then $p(x)=x[0]$ but $p\left(z^{N}\right)=\overline{0}$ for all $N$. Hence, $p$ is not continuous.

(f) Let $(Y, S)$ be any CP subshift. Let $\left\{w_{i}\right\}$ count the finite words in $Y$. Then $Y \cup \bigcup\left\{\overline{w_{i}}\right\}$ is a $\mathrm{CP}$ subshift with dense periodic points.

If $(X, T)$ is a metrizable $\mathrm{CT}$ then it is chain transitive, because the fixed point $e$ lies in $\omega T(x) \cap \alpha T(x)$ for every $x \in X$. So if $x_{1}, x_{2} \in X$, there is an $\epsilon$ chain from $x_{1}$ to $e$ and from $e$ to $x_{2}$ for every $\epsilon>0$. Hence, we can attach a single orbit of isolated points and obtain a metrizable CT, $\left(X^{*}, T\right)$ which is topologically transitive so that of $X^{*}$ the disjoint union of $X$ and the dense orbit of isolated points $O_{T}\left(x^{*}\right)$. See Example 1.5.

Example 1.19. It may happen that we cannot choose the extension so that $\left(X^{*}, T\right)$ is WAP.

Let $(X, T)$ be a CT WAP with fixed point $e$ and which is not semi-trivial. That is, there exists $p$ in the $A(X, T)$ with $p \neq u$ and so $p(X) \backslash\{e\}$ is nonempty. Let $\bar{X}=X_{1} \vee X_{2}$, two copies of $X$ with the fixed points identified. For any 
map $g$ on $X$ which fixes $e$, let $\bar{g}$ on $\bar{X}$ be copies of $g$ on each term. The system $(\bar{X}, \bar{T})$ is clearly a CT WAP and $p \mapsto \bar{p}$ is an isomorphism from $E(X, T)$ onto $E(\bar{X}, \bar{T})$. Notice the $\bar{p}(\bar{X}) \backslash X_{i}$ is nonempty for $i=1,2$.

Now let $\left(X^{*}, T^{*}\right)$ contain $(\bar{X}, \bar{T})$ and with $X^{*} \backslash \bar{X}$ consisting of a single dense orbit $O_{T^{*}}\left(x^{*}\right)$.

Let $q$ be an element of the enveloping semigroup of $E\left(X^{*}, T^{*}\right)$ with $q\left(x^{*}\right) \in$ $X_{1}$. Then $q$ maps the whole orbit of $x^{*}$ into $X_{1}$ and if $q$ is continuous then $q\left(X^{*}\right) \subset X_{1}$. Thus, every continuous element of the enveloping semigroup $E\left(X^{*}, T^{*}\right)$ maps all of $X^{*}$ either into $X_{1}$ or into $X_{2}$. Every element of the enveloping semigroup of $(\bar{X}, \bar{T})$ extends to some element of the enveloping semigroup of $\left(X^{*}, T^{*}\right)$. Thus, if $p^{*}$ extends $\bar{p}$ it cannot be continuous and so $\left(X^{*}, T^{*}\right)$ is not WAP.

We will say that $A(X, T)$ distinguishes points when $p\left(x_{1}\right)=p\left(x_{2}\right)$ for all $p \in A(X, T)$ implies $x_{1}=x_{2}$. It suffices that some $p \in A(X, T)$ be injective. If $X$ has any non-trivial, but semi-trivial subspace then $A(X, T)$ does not distinguish points.

Let $T_{*}$ be composition with $T$ on $E(X, T)$. Clearly $i d_{X}$ is a transitive point for $T_{*}$. If $(X, T)$ is not weakly rigid, i.e. $i d_{X}$ is not a recurrent point for $T_{*}$, then $i d_{X}$ is an isolated transitive point for $T_{*}$ and $A(X, T)=R_{T_{*}}\left(i d_{X}\right)$ is a proper subset of $E(X, T)$.

Now we assume that $\left(X^{*}, T\right)$ is obtained from $(X, T)$ as above by attaching a single dense orbit of isolated points. Assume that $x^{*}$ is a transitive point for $\left(X^{*}, T\right)$. Then $e v_{x^{*}}:\left(E\left(X^{*}, T\right), T_{*}\right) \rightarrow\left(X^{*}, T\right)$ is a factor map sending $A\left(X^{*}, T\right)$ to $X=R_{T}\left(x^{*}\right)$. If $\left(X^{*}, T\right)$ is WAP then the map is an isomorphism by Proposition 1.8.

Now assume that the subspace $(X, T)$ is WAP. As is true for any subsystem the restriction map $\rho: A\left(X^{*}, T\right) \rightarrow A(X, T)$ is surjective.

Proposition 1.20. Assume that $\left(X^{*}, T\right)$ is topologically transitive with an isolated transitive point $x^{*}$ such that the subsystem $(X, T)$ with $X=R_{T}\left(x^{*}\right)$ is $W A P$. The map $\rho$ is injective, and so is an isomorphism, iff $\left(X^{*}, T\right)$ is WAP and, in addition, $A(X, T)$ distinguishes points of $X$.

Proof: Since $X$ is WAP, $A(X, T)$ is abelian. If $\rho$ is injective then $A\left(X^{*}, T\right)$ is abelian and so $\left(X^{*}, T\right)$ is WAP by Proposition 1.8 .

Now assume $\left(X^{*}, T\right)$ is WAP. We show that $\rho$ is injective iff $A(X, T)$ distinguishes the points of $X$.

Let $p_{1}, p_{2} \in A\left(X^{*}, T\right)$. Since $A\left(X^{*}, T\right)$ is abelian, $p_{1}\left(q\left(x^{*}\right)\right)=p_{2}\left(q\left(x^{*}\right)\right)$ for all $q \in A\left(X^{*}, T\right)$ iff $q\left(p_{1}\left(x^{*}\right)\right)=q\left(p_{2}\left(x^{*}\right)\right)$ for all $q \in A\left(X^{*}, T\right)$. The first says $\rho\left(p_{1}\right)=\rho\left(p_{2}\right)$ and the second says $p_{1}\left(x^{*}\right), p_{2}\left(x^{*}\right) \in X_{1}$ are not distinguished 
by $A(X, T) . \rho$ is injective says that the first implies $p_{1}=p_{2}$ while $A(X, T)$ distinguishes points says that the second implies $p_{1}\left(x^{*}\right)=p_{2}\left(x^{*}\right)$ and so by continuity $p_{1}=p_{2}$. This proves the equivalence.

Corollary 1.21. Assume $(X, T)$ is $W A P$, is not weakly rigid and is such that $A(X, T)$ distinguishes points. If there exists $\left(X^{*}, T\right)$ topologically transitive with an isolated transitive point $x^{*}$ such that $(X, T)$ is the subsystem with $X=R_{T}\left(x^{*}\right)$ then $\left(X^{*}, X, T\right)$ is isomorphic to $\left(E(X, T), A(X, T), T_{*}\right)$.

Proof: Since $X$ is not weakly rigid, $\rho$ is an isomorphism from $\left(E\left(X^{*}, T\right), A\left(X^{*}, T\right), T_{*}\right)$ onto $\left(E(X, T), A(X, T), T_{*}\right)$. On the other hand, $e v_{x^{*}}$ is an isomorphism from $\left(E\left(X^{*}, T\right), A\left(X^{*}, T\right), T_{*}\right)$ onto $\left(X^{*}, X, T\right)$.

\subsection{Coalescence, LE, HAE and CT-WAP systems.}

Given a metric dynamical system $(X, T)$, a point $x \in X$ is an equicontinuity point if for every $\epsilon>0$ there is $\delta>0$ such that $d\left(x^{\prime}, x\right)<\delta$ implies $d\left(T^{n} x^{\prime}, T^{n} x\right)<\epsilon$ for every $n \in \mathbb{Z}$. A system $(X, T)$ is called equicontinuous if it is a metric system and every point in $X$ is an equicontinuity point (and then it is already uniformly equicontinuous meaning that the $\delta$ in the above definition does not depend on $x$ ). It is called almost equicontinuous, hereafter AE, when it is a metric system and there is a dense set of points in $X$ at which $\left\{T^{n}: n \in \mathbb{Z}\right\}$ is equicontinuous. Following [21] we will call $(X, T)$ hereditarily almost equicontinuous, hereafter HAE, when every subsystem (i.e. closed invariant subset) is again an AE system. As was shown in [5] every metrizable WAP is HAE (see also [18, Chapter 1, Sections 8 and 9]).

An isolated point in a metric system is an equicontinuity point and so if the isolated points are dense then the system is AE. Hence, if $(X, T)$ is countable then every subsystem is AE i.e. $(X, T)$ is HAE.

A system $(X, T)$ is expansive if it is a metric system and there exists $\epsilon>0$ such that for every $x_{1} \neq x_{2}, d\left(T^{n}\left(x_{1}\right), T^{n}\left(x_{2}\right)\right)>\epsilon$ for some $n \in \mathbb{Z}$. Any subshift is expansive. The following is obvious.

Lemma 1.22. If $(X, T)$ is expansive then $x \in X$ is an equicontinuity point iff it is isolated.

From this follows the result from [22] that a subshift is HAE iff it is countable. 
Proposition 1.23. An expansive dynamical system $(X, T)$ is $H A E$ iff $X$ is countable. In particular, a subshift is HAE iff it is countable.

Proof: It was observed above that a countable system is HAE.

Now assume that $X$ is uncountable and so contains a Cantor set $C$. If $X_{1}$ is the closure of $\bigcup_{n}\left\{T^{n}(C)\right\}$, then the subsystem $\left(X_{1}, T\right)$ is expansive and contains no isolated points. So by Lemma 1.22 it has no equicontinuity points. Thus, $(X, T)$ is not HAE.

Thus:

Proposition 1.24. A WAP subshift is countable.

Following [25] we call $(X, T)$ locally equicontinuous (hereafter LE) if it is a metric system and each point $x$ is an equicontinuity point in its orbit closure or, equivalently, if each orbit closure is an almost equicontinuous subsystem. The equivalence follows from the Auslander-Yorke Dichotomy Theorem, [10], which says that in a topologically transitive system the set of equicontinuity points either coincides with the set of transitive points or else it is empty.

Remark 1.25. From the latter condition, it follows that an HAE system is LE. Any CP system is LE since each point is isolated in its orbit closure. From Proposition 1.23 it follows that any uncountable CP subshift is LE but not HAE.

A system $(X, T)$ is coalescent when any surjective action map $\pi$ on $(X, T)$ is an isomorphism.

Proposition 1.26. A topologically transitive metric system which is WAP is coalescent.

Proof: Let $x^{*}$ be a transitive point. There exists $p$ in the enveloping semigroup with $p\left(x^{*}\right)=\pi\left(x^{*}\right)$. Because $p$ is continuous it is an action map and so since $p$ and $\pi$ agree on the dense orbit of $x^{*}, p=\pi$. Since $p$ is surjective, $p\left(x^{*}\right)$ is a transitive point and so there exists $q$ such that $q p\left(x^{*}\right)=x^{*}$ and so $q p=i d$. Hence, $p$ is injective with inverse $q$.

Example 1.27. In general a WAP system need not be coalescent. 
If $(X, T)$ is WAP then the countable product $\left(X^{\mathbb{N}}, T^{\mathbb{N}}\right)$ is WAP and the shift map is a surjective action map which is not injective. If $X$ is CT with fixed point $e$ then the infinite wedge which is $\left\{x \in X^{\mathbb{N}}: x_{i} \neq e\right.$ for at most one $\left.i\right\}$ is a closed invariant set which is shift invariant as well. This is also WAP and not coalescent. In addition, it is countable if $X$ is.

Lemma 1.28. If a dynamical system $(X, T)$ contains an increasing net of topologically transitive subsystems $\left\{X^{i}\right\}$ with $\bigcup_{i}\left\{X^{i}\right\}$ dense in $X$, then $X$ is also topologically transitive.

Proof: Let $U, V \subset X$ be two nonempty open subsets. For some $i$

$$
U \cap X^{i} \neq \emptyset \text { and } V \cap X^{i} \neq \emptyset .
$$

As $X^{i}$ is topologically transitive, there exists $k \in \mathbb{Z}$ with $T^{k}\left(U \cap X^{i}\right) \cap(V \cap$ $\left.X^{i}\right) \neq \emptyset$ and, a fortiori, also $T^{k}(U) \cap V \neq \emptyset$.

Proposition 1.29. Every dynamical system is a union of maximal topologically transitive subsystems.

Proof: Let $(X, T)$ be a dynamical system and consider the family $\mathcal{T}$ of topologically transitive subsystems of $X$. Using Lemma 1.28 it is easy to check that this family is inductive. Hence, by Zorn's Lemma, every topologically transitive subsystem of $X$ is contained in a maximal element of $\mathcal{T}$. In particular, for $x \in X$, the orbit closure of $X$ is contained in a maximal element of $\mathcal{T}$.

We use this proposition to obtain the following results on $E$-Coalescence (i.e. the property that every continuous surjective element of $E(X, T)$ is injective).

Recall that a dynamical system $(X, T)$ is called (i) weakly rigid if there is a net $\left\{T^{n_{i}}\right\}$ with $\left|n_{i}\right| \rightarrow \infty$ and $\left\{T^{n_{i}}(x)\right\} \rightarrow x$ for every $x \in X$, or, equivalently, if $i d_{X} \in A(X, T)$. (ii) rigid if the net can be chosen to be a sequence, and (iii) uniformly rigid if the convergence can be taken to be uniform (see [20]). Recall that if $X$ is a topologically transitive AE system, and a fortiori a metrizable, topologically transitive WAP, then it is uniformly rigid (see [22], [24] and [4]). Theorem 1.30. Let $(X, T)$ be an AE system. Assume that $p \in A(X, T)$ is continuous and surjective.

(i) If $(X, T)$ is topologically transitive then $p$ is injective and so is an isomorphism. If $T^{n_{j}}$ is a net converging pointwise to $p$ then it converges uniformly to $p$ and $T^{-n_{j}}$ converges uniformly to $p^{-1}$. Thus, $p^{-1}, i d_{X} \in$ $E(X, T)=A(X, T)$. 
(ii) If $X_{1}$ is a maximal topologically transitive subset of $X$ then $p\left(X_{1}\right)=X_{1}$.

(iii) If $(X, T)$ is HAE then $p$ is an isomorphism and if $T^{n_{j}}$ is a net converging pointwise to $p$ then $T^{-n_{j}}$ converges pointwise to $p^{-1}$. Thus, $p^{-1}, i d_{X} \in E(X, T)=A(X, T)$ and the system is weakly rigid.

Proof: (i) Let $x^{*}$ be a transitive point for $X$. Since $p$ is surjective and continuous, $p x^{*}$ is a transitive point and so there exists a sequence $T^{n_{i}}$ with $T^{n_{i}} p x^{*}$ converging to $x^{*}$. Let $\epsilon>0$. By the Auslander-Yorke Dichotomy Theorem, [10] $x^{*}$ is an equicontinuity point and for sufficiently large $i, T^{n_{i}} p T^{k} x^{*}=T^{k} T^{n_{i}} p x^{*}$ is within $\epsilon$ of $T^{k} x^{*}$ for all $k$. Since the orbit of $x^{*}$ is dense it follows that $T^{n_{i}} p$ converges uniformly to $i d_{X}$. Hence, $p$ is injective and so is an isomorphism by compactness. If $q$ is any limit point of $T^{n_{i}}$ in $E(X, T)$ then continuity of $p$ implies that $p q$, which is the limit of $p T^{n_{i}}=T^{n_{i}} p$, is the identity, and so $q=p^{-1}$. Thus, $p^{-1} \in E(X, T)$. Hence, $i d_{X}=p^{-1} p \in A(X, T)$ and so $(X, T)$ is weakly rigid and $E(X, T)=A(X, T)$. Since $p T^{n_{i}}=T^{n_{i}} p$ converges uniformly to $p p^{-1}$, uniform continuity of $p^{-1}$ implies $T^{n_{i}}$ converges uniformly to $p^{-1}$. Hence, $\lim _{i, j \rightarrow \infty} T^{n_{i}-n_{j}}=i d_{X}$ and so the system is uniformly rigid. Finally, if a net $T^{m_{i}}$ converges to $p$ pointwise then $p^{-1} T^{m_{i}} x^{*}$ is eventually close to $x^{*}$ and so, as above, $p^{-1} T^{m_{i}}$ converges to $i d_{X}$ uniformly. It follows that $T^{m_{i}}$ converges to $p$ uniformly and $T^{-m_{i}}$ converges uniformly to $p^{-1}$.

(ii) Let $x^{*}$ be a transitive point for $X_{1}$. Since $p$ is surjective, there exists $x_{1} \in X$ such that $p x_{1}=x^{*}$. The orbit closure of $x_{1}$ is a topologically transitive subset of $X$ and it contains $p x_{1}=x^{*}$ and so contains $X_{1}$, which is the orbit closure of $x^{*}$. Hence, by maximality, $X_{1}=\overline{O_{T}\left(x_{1}\right)}$. Since $p$ is a continuous action map, $p\left(X_{1}\right)=p\left(\overline{O_{T}\left(x_{1}\right)}\right)=\overline{O_{T}\left(x^{*}\right)}=X_{1}$.

(iii) By 1.29 each point is contained in a maximal topologically transitive subset of $X$, which is necessarily closed.

Now if for some points $x_{1}, x_{2} \in X$ we have $z=p x_{1}=p x_{2}$, then let $X_{i}$ be a maximal topologically transitive subset of $X$ which contains $x_{i}$. Since each $X_{i}$ is closed and invariant, $z \in X_{1} \cap X_{2}$. By (ii) $p$ is surjective on each $X_{i}$ and so by (i) there exist $q_{i} \in A(X, T)$ such that on $X_{i} q_{i} p$ is the identity. In particular, $q_{i} z=x_{i}$. Hence, $q_{2} p x_{1}=x_{2}$ and so $x_{2}$ as well as $x_{1}$ is in $X_{1}$. Since $q_{1} p$ is the identity on $X_{1}$ it follows that $x_{1}=x_{2}$. Thus, $p$ is an isomorphism. Now let $T^{m_{i}}$ be a net converging to $p$ pointwise. By part (i) $T^{-m_{i}}$ converges to $p^{-1}$ uniformly on each orbit closure and so pointwise on $X$. Hence, $p^{-1}$ and $i d_{X}=p^{-1} p$ are in $A(X, T)$. Hence, $E(X, T)=A(X, T)$ and $(X, T)$ is weakly rigid.

Corollary 1.31. Every metrizable WAP dynamical system is E-coalescent. 
Proof: If $(X, T)$ is a metrizable WAP dynamical system then (i) it is HAE, and (ii) every $p \in E(X, T)$ is continuous. Now apply Theorem 1.30.

Corollary 1.32. Let $(X, T)$ be a WAP system and $p \in A(X, T)$.

The restriction of $p$ to the subsystem $Z=\bigcap_{n \in \mathbb{N}} p^{n} X$ is surjective on $Z$ and so is an automorphism of $Z$. In particular the system $(Z, T)$ is weakly rigid.

If, in addition, $(X, T)$ is $C P$, then every point of $Z$ is periodic. If, moreover, $(X, T)$ is topologically transitive then $Z$ consists of a single periodic orbit which is the unique minimal subset of $X$ and is independent of the choice of $p \in$ $A(X, T)$.

Proof: Assume first that $X$ is metrizable. Hence, $(X, T)$ is HAE because it is WAP.

Clearly $Z$ is a (nonempty) subsystem and $p Z=Z$. Thus, by Theorem 1.30 $p \mid Z$ is an automorphism of $Z$. Since $p \in A(X, T)$ it follows that $Z$ is weakly rigid and so every point of $Z$ is recurrent. So if the system is CP then every point of $Z$ is periodic.

If, in addition, $X$ is transitive then by Corollary $1.9 X$ contains a unique minimal subset and so $Z$ consists of a single periodic orbit which is that minimal subset.

Since the group $\mathbb{Z}$ is countable, $(X, T)$ is an inverse limit of a net $\left\{\left(X^{i}, T^{i}\right)\right\}$ of metrizable systems which are WAP since the latter property is preserved by factors. The set $Z$ projects onto the corresponding set $Z^{i}$. Thus, the restriction of $p$ to $Z$ is the inverse limit of isomorphisms and so is an isomorphism. The inverse limit of weakly rigid systems is weakly rigid. So in the CP case the points of $Z$ are periodic. Having a unique minimal subset is preserved by inverse limits and so if $X$ is transitive then $Z$ is a single periodic orbit as before.

\section{Questions 1.33.}

(1) Is there a metric WAP system which is central, but which is not rigid?

(2) If a homeomorphism for $X$ is in $A(X, T)$ then is its inverse also in $A(X, T)$ (and so it is weakly rigid)? For WAP or even for HAE the answer is yes, by Theorem 1.30 above.

Lemma 1.34. Assume $(X, T)$ is a CP system. If there is an infinite sequence $\left\{x_{i}: i \in \mathbb{N}\right\}$ in $X$ such that $x_{i} \in R_{T}\left(x_{i+1}\right)$ for all $i$, then $x_{1}$ is a periodic point and all $x_{i}$ 's are in the orbit of $x_{1}$. 
Proof:

First assume that $X$ is metrizable.

If $x_{i}$ is periodic then all $x_{j}$ 's with $j<i$ are in the orbit of $x_{i}$. Hence, if infinitely many of the $x_{i}$ 's are periodic then they all are and all lie in the same periodic orbit. We show that the alternative to infinitely many periodic points cannot happen.

If instead there are only finitely many periodic points in the sequence then by omitting finitely many initial terms and re-numbering we can assume that none are periodic. Let $A_{i}$ be the orbit closure of $x_{i}$.

If $x_{i+1} \in R_{T}\left(x_{i+1}\right)$ then it is positively or negatively recurrent. Since $X$ is $\mathrm{CP}$ the only recurrent points are periodic. Hence, if $x_{i+1}$ is not periodic then $x_{i+1}$ is not in the orbit closure of $x_{i}$ which we label $A_{i}$. Since $x_{i} \in A_{i+1}$ we have $A_{i} \subset A_{i+1}$. Since $x_{i+1} \in A_{i+1} \backslash A_{i}$, each inclusion is strict. Each $A_{i}$ is topologically transitive with transitive point $x_{i}$. Therefore the closure of the union $A=\overline{U A_{i}}$ is topologically transitive. Let $z$ be a transitive point for $A$. It is isolated in $A=\overline{\bigcup A_{i}}$ and so must lie in some $A_{j}$, but since the $\left\{A_{i}\right\}$ sequence is a strictly increasing sequence of closed invariant sets, it cannot be in any of them.

For the general case, we can assume that some $x_{i}$ is not periodic. There is a metrizable factor for which the image of $x_{i}$ is not periodic and this contradicts the metric result above.

Proposition 1.35. Assume that $(X, T)$ is a CP, WAP system. If $\left\{p_{i}: i \in \mathbb{N}\right\}$ is a sequence in $A(X, T)$ then $\bigcap_{n=1}^{\infty} p_{n} p_{n-1} \cdots p_{1} X$ is a closed subset consisting of periodic points.

Proof: For $i=1,2, \ldots$ and $n \geq i$ let $X_{i, n}=p_{i} p_{i+1} \cdots p_{n} X . X_{i}=\bigcap_{n=i}^{\infty} X_{i, n}$. Since each $p_{i}$ is continuous, each $X_{i, n}$ is a closed, invariant subspace and the sequence is decreasing in $n$ and $p_{i}\left(X_{i+1, n}\right)=X_{i, n}$ when $n \geq i+1$. Hence, continuity and compactness imply that $p_{i}\left(X_{i+1}\right)=X_{i}$. Since the semigroup is abelian, $X_{i, n}=p_{n} p_{n-1} \cdots p_{i} X$. Let $x_{1} \in X_{1}$. By induction we can build a sequence $x_{i} \in X_{i}$ such that $p_{i}\left(x_{i+1}\right)=x_{i}$ and so $x_{i} \in R_{T}\left(x_{i+1}\right)$. From Lemma 1.34 it follows that $x_{1}$ is periodic.

Corollary 1.36. Assume that $(X, T)$ is a CP, WAP system. If $\left\{x_{i}: i \in \mathbb{N}\right\}$ is a sequence in $X$ such that $x_{i+1} \in R_{T}\left(x_{i}\right)$ for all $i$, then $\bigcap_{i} \overline{O\left(x_{i}\right)}$ (the orbit closures of the $x_{i}$ 's) is a closed subset consisting of periodic points.

Proof: The sequence $\left\{\overline{O\left(x_{i}\right)}\right\}$ is decreasing and so we can restrict to the case $X=\overline{O\left(x_{1}\right)}$. This is a topologically transitive WAP system. By assumption, 
there exist $p_{i} \in A(X, T)$ such that $p_{i}\left(x_{i}\right)=x_{i+1}$. In the notation of the proof of Proposition 1.35, $x_{n+1} \in X_{1, n}$ for all $n \geq 1$. Hence, $\bigcap_{n} \overline{O\left(x_{n}\right)} \subset \bigcup_{n} X_{1, n}$ and the latter consists of periodic points by Proposition 1.35 .

Recall that for $(X, T)$ a cascade, we defined the relation $R_{T}$ on $X$ with $R_{T}(x)$ the set of limit points of the bi-infinite orbit sequence $\left\{T^{i}(x): i \in \mathbb{Z}\right\}$. For $A \subset X$ we let $R_{T}^{*}(A)=\left\{x: R_{T}(x) \subset A\right\}$. Let $R_{T}^{* n}(A)=R_{T}^{*}\left(R_{T}^{*(n-1)}(A)\right)=$ $\left\{x: R_{T}^{n}(x) \subset A\right\}$. Observe that $R_{T}^{*}(A)=\bigcap\left\{p^{-1}(A): p \in A(X, T)\right\}$. Hence, in the WAP case with all members of $A(X, T)$ continuous, $R_{T}^{*}(A)$ is closed when $A$ is.

A subset $A \subset X$ is called orbit-closed if $x \in A$ implies $\overline{O(x)} \subset A$.

An orbit-closed set is clearly invariant, but need not be closed. On the other hand, a closed, invariant set is orbit-closed. In general, a set $A$ is orbitclosed iff it is invariant and $x \in A$ implies $R_{T}(x) \subset A$. Thus, a subset $A$ is orbit-closed iff it is invariant and $A \subset R_{T}^{*}(A)$.

Lemma 1.37. Let $A \subset X$. The set $R_{T}^{*}(A)$ is orbit-closed, i.e.

$$
x \in R_{T}^{*}(A) \quad \Rightarrow \quad \overline{O(x)} \subset R_{T}^{*}(A)
$$

Proof: Since $R_{T}\left(T^{i}(x)\right)=R_{T}(x)$ for all $i \in \mathbb{Z}$ it follows that $R_{T}^{*}(A)$ is invariant. Since $R_{T} \circ R_{T} \subset R_{T}, y \in R_{T}(x)$ implies $R_{T}(y) \subset R_{T}(x)$. It follows that $x \in R_{T}^{*}(A)$ implies $y \in R_{T}^{*}(A)$.

We will say that $A$ is limit determined or L-determined if it is invariant and $x \in A$ iff $R_{T}(x) \subset A$. That is, $A$ is L-determined when it is orbit closed and $R_{T}(x) \subset A$ only when $x \in A$. Thus, a subset $A$ is L-determined iff it is invariant and $A=R_{T}^{*}(A)$. Clearly,

$$
B \subset A \text { and } A \text { L-determined } \quad \Longrightarrow \quad R_{T}^{*}(B) \subset A \text {. }
$$

The entire space $X$ is L-determined. For a collection $\left\{A_{i}\right\}$ of subsets of $X$, the intersection is $\bigcap_{i} A_{i}$ invariant, or orbit-closed or L-determined, if each $A_{i}$ satisfies the corresponding property. The union $\bigcup_{i} A_{i}$ is invariant or orbitclosed if each $A_{i}$ satisfies the corresponding property.

It follows that for any set $A$ there is a minimum L-determined set which contains it, i.e. the intersection of all L-determined sets containing it. For an orbit-closed set, we can obtain this set via the usual transfinite construction. 
Assume that $A \subset X$ is orbit-closed. Define

$$
\begin{aligned}
& z_{0}^{*}(A)=A, \\
& z_{\alpha+1}^{*}(A)=R_{T}^{*}\left(z_{\alpha}^{*}(A)\right), \\
& z_{\beta}^{*}(A)=\bigcup_{\alpha<\beta} z_{\alpha}^{*}(A),
\end{aligned}
$$

for $\beta$ a limit ordinal. Every $z_{\alpha}^{*}$ is orbit-closed by Lemma 1.37 and so the sequence is increasing until it stabilizes at the minimum L-determined set which contains $A$.

A closed invariant set $K \subset X$ is an isolated invariant set if there is an open set $U$ containing $K$ such that $K$ is the maximum invariant subset of $U$, i.e. $K=\bigcap_{i \in \mathbb{Z}} T^{i}(U)$. If $\left\{K_{n}\right\}$ is a decreasing sequence of closed invariant sets with intersection $K$ isolated, then $K_{n} \subset U$ implies $K_{n}=K$ and so eventually $K_{n}=K$. In an expansive system, like a subshift, any fixed point is an isolated invariant set.

Lemma 1.38. Let $(X, T)$ be a $C T$, WAP subshift with fixed point $\{e\}$. For any infinite sequence $p_{1}, p_{2}, \ldots$ of elements of $A(X, T)$ there there exists an $n$ such that $p_{1} p_{2} \cdots p_{n}(X)=\{e\}$.

Proof: Proposition 1.35 implies that $\{e\}$ is the intersection of the decreasing sequence $\left\{p_{1} p_{2} \cdots p_{n}(X)\right\}$. If $V$ is any neighborhood of $e$ then the invariant set $p_{1} p_{2} \cdots p_{n}(X)$ is contained in $V$ for sufficiently large $n$. Since $e$ is an isolated invariant set, we can choose $V$ so that $\{e\}$ is the only invariant subset of $V$.

Now we restrict to the case where $(X, T)$ is a metrizable CT, WAP with a single minimal set consisting of a fixed point $e$. We will call such a system $C T-W A P$.

Fix a closed neighborhood $V(e)$ of $e$. let $A(e)$ denote the maximal invariant subset of $X$ which is contained in $V(e)$, i.e.

$$
A(e)=\bigcap_{i \in \mathbb{Z}} T^{i}(V(e))
$$

If $V(e)$ is clopen, $A(e)$ is an isolated invariant set. If $\{e\}$ is an isolated invariant set, e.g. if $(X, T)$ is a subshift, then we choose $V(e)$ so that $A(e)=$ $\{e\}$.

Proposition 1.39. Assume $(X, T)$ is $C T$-WAP. Let $A$ be any invariant set which contains $A(e)$. If $x \notin A$ then there exists $q \in E(X, T)$ such that $q x \notin$ $A \cup V(e)$ but for all $p \in A(X, T), p q x \in A$, i.e. $R_{T}(q x)=q\left(R_{T}(x)\right) \subset A$. That is, $q x \in R_{T}^{*}(A) \backslash(A \cup V(e))$. 
Proof: Clearly,

$$
A \subset \bigcap_{\ell \in \mathbb{Z}} T^{\ell}(A \cup V(e)) \subset A \cup A(e)=A
$$

So for some $i \in \mathbb{Z}, T^{i}(x) \notin A \cup V(e)$. If $R_{T}\left(T^{i}(x)\right) \subset A \cup V(e)$ then let $q=T^{i}$. Otherwise, there exists $q_{1}$ such that $q_{1}\left(T^{i}(x)\right) \notin A \cup V(e)$. Continue inductively defining $q_{n}$ such that $q_{n} \cdots q_{1}\left(T^{i}(x)\right) \notin A \cup V(e)$ whenever $R_{T}\left(q_{n-1} \cdots q_{1}\left(T^{i}(x)\right)\right.$ is not contained in $A \cup V(e)$. This process must terminate.

Assume it did not. By Proposition $1.35\{e\}=\bigcap_{n}\left\{q_{n} \cdots q_{1}(X)\right\}$ and so eventually $q_{n} \cdots q_{1}(X)$ is a closed invariant set contained in $V(e)$. By invariance, $q_{n} \cdots q_{1}(X) \subset A(e)$, contradicting the definition of $q_{n}$.

If $q_{n} \cdots q_{1}\left(T^{i}(x)\right) \notin A \cup V(e)$ but $R_{T}\left(q_{n} \cdots q_{1}\left(T^{i}(x)\right) \subset A \cup V(e)\right.$, then let $q=q_{n} \cdots q_{1} T^{i}$. Since $R_{T}(q x) \subset A \cup V(e)$ and $R_{T}(q x)$ is an invariant set, equation 1.8 implies $R_{T}(q x) \subset A$.

Since the system is WAP, $q$ is an action map and so $q\left(R_{T}(x)\right)=R_{T}(q x)$.

Proposition 1.40. Assume $(X, T)$ is $C T$-WAP. Let $A$ be any orbit-closed set which contains $A(e)$ and let $\left\{z_{\alpha}^{*}(A)\right\}$ be the sequence defined by (1.6). For any ordinal $\alpha$, if $x \notin z_{\alpha}^{*}(A)$ then there exists $q \in E(X, T)$ such that

$$
\begin{array}{ll}
q x \notin z_{\alpha}^{*}(A) \cup V(e) \quad \text { and } \quad R_{T}(q x) \subset z_{\alpha}^{*}(A) \\
\text { i.e. } \quad q x \in z_{\alpha+1}^{*}(A) \backslash\left(z_{\alpha}^{*}(A) \cup V(e)\right) .
\end{array}
$$

For any such $q, R_{T}(q x)$ meets $z_{\beta+1}^{*}(A) \backslash\left(z_{\beta}^{*}(A) \cup V(e)\right)$ for every $\beta<\alpha$.

Proof: We repeatedly apply Proposition 1.39. First we obtain $q \in E(X, T)$ which satisfies (1.9).

For any $\beta<\alpha$ there exists $q_{1} \in E(X, T)$ such that $q_{1} q x \in z_{\beta+1}^{*}(A) \backslash\left(z_{\beta}^{*}(A) \cup\right.$ $V(e))$. Since $\beta+1 \leq \alpha, q x \notin z_{\beta+1}^{*}(A)$ and so $T^{i}(q x) \notin z_{\beta+1}^{*}(A)$ for any $i \in \mathbb{Z}$. Hence, $q_{1} \in A(X, T)$ and so $q_{1}(q x) \in R_{T}(q x)$.

Corollary 1.41. Assume $(X, T)$ is CT-WAP. Let $A$ be any orbit-closed set which contains $A(e)$ and let $\left\{z_{\alpha}^{*}(A)\right\}$ be the sequence defined by (1.6). If for any ordinal $\alpha, z_{\alpha}^{*}(A)$ is a proper subset of $X$ then $z_{\alpha+1}^{*}(A) \backslash z_{\alpha}^{*}(A)$ is nonempty. So the transfinite sequence $\left\{z_{\alpha}^{*}(A)\right\}$ is strictly increasing until the first ordinal $\alpha^{*}$ such that $z_{\alpha^{*}}^{*}(A)=X$. If $(X, T)$ is topologically transitive with transitive point $x^{*}$ then $\alpha^{*}$ is the first ordinal such that $x^{*} \in z_{\alpha^{*}}^{*}(A)$ and $\alpha^{*}$ is a non-limit ordinal.

Proof: That the sequence is strictly increasing until $z_{\alpha}^{*}(A)=X$ is clear from Proposition 1.40. It is also clear that if $x^{*}$ is a transitive point then 
$x^{*} \in z_{\alpha}^{*}(A)$ implies $X=\overline{O\left(x^{*}\right)} \subset a_{\alpha}$ by (1.4) and so $z_{\alpha}^{*}(A)=X$ iff $x^{*} \in z_{\alpha}^{*}(A)$. If $x^{*} \in \bigcup_{\beta<\alpha} z_{\beta}^{*}(A)$ then $x^{*} \in a_{\beta}(A)$ for some $\beta<\alpha$ and so $\alpha^{*}$ cannot then be a limit ordinal.

For the case when $(X, T)$ is a CT-WAP with the fixed point $e$ isolated (as a closed invariant subset), e.g. a CT-WAP subshift, we have chosen $V(e)$ so that $A(e)=\{e\}$ and we let $A=A(e)=z_{0}^{*}(e)=\{e\}$. We then call the ordinal at which the $z_{\alpha}^{*}(e)$ sequence stabilizes the height* of $(X, T)$. Because $X$ is then countable it follows that the ordinal $\alpha^{*}$ is countable.

Corollary 1.42. Assume $(X, T)$ is CT-WAP. If the fixed point e is an isolated invariant set then the entire space $X$ is the only L-determined subset of $X$.

Proof: The transfinite sequence $\left\{z_{\alpha}^{*}(e)\right\}$ stabilizes at $X$ by Corollary 1.41. If $A$ is any L-determined set then for any $x \in A, e \in R_{T}(x) \subset A$ since $A$ is orbit-closed. Hence, $z_{0}^{*}(e) \subset A$ and so inductively $z_{\alpha}^{*}(e) \subset A$ for all $\alpha$. Hence, $A=X$.

\subsection{Discrete suspensions and spin constructions.}

For any $(X, T)$ and positive integer $N$ we define on $X \times[0, N-1]$ the homeomorphism $\tilde{T}$ by

$$
\begin{aligned}
& \tilde{T}(x, i)= \begin{cases}(x, i+1) & \text { for } \quad i<N-1, \\
(T(x), 0) & \text { for } \quad i=N-1 .\end{cases} \\
& \text { so that } \tilde{T}^{N}=T \times i d_{[0, N-1]} \text {. }
\end{aligned}
$$

$(X \times[0, N-1], \tilde{T})$ is called the discrete $N$ step suspension. It is countable if $X$ is, it is $\mathrm{CP}$ if $(X, T)$ is $\mathrm{CT}$. It is WAP if $(X, T)$ is. Apply the following

Lemma 1.43. A point $x \in X$ is an equicontinuity point for $T$ on $X$ iff it is an equicontinuity point for $T^{N}$ on $X$. Hence, $(X, T)$ is $A E$ or $H A E$ iff $\left(X, T^{N}\right)$ is. In general, $(X, T)$ is WAP iff $\left(X, T^{N}\right)$ is.

Proof: Since $\left\{T^{N i}: i \in \mathbb{Z}\right\} \subset\left\{T^{i}: i \in \mathbb{Z}\right\}$, an equicontinuity point for $T$ is one for $T^{N}, E\left(X, T^{N}\right) \subset E(X, T)$ and so each of the conditions for $T$ implies 
the corresponding condition for $T^{N}$. In fact,

$$
\begin{aligned}
\left\{T^{i}: i \in \mathbb{Z}\right\} & =\left\{T^{N i} \circ T^{k}: i \in \mathbb{Z}, k=0, \ldots, N-1\right\} \\
& =\bigcup_{k=0}^{N-1} T^{k} \circ\left\{T^{N i}: i \in \mathbb{Z}\right\} .
\end{aligned}
$$

It follows that if $x$ is an equicontinuity point for $T^{N}$ then it is for $T$. Also, we obtain $E(X, T)=\bigcup_{k=0}^{N-1} T^{k} \circ E\left(X, T^{N}\right)$ and so the elements of $E(X, T)$ are continuous when those of $E\left(X, T^{N}\right)$ are.

Theorem 1.44. If $(X, T)$ is a CP WAP system with a unique minimal set, a periodic orbit of period $N$, then $(X, T)$ is isomorphic to the discrete $N$ step suspension of a CT WAP.

Proof: Let $\left\{x_{0}, \ldots, x_{N-1}\right\}$ be the periodic orbit in $X$. By Lemma 1.43 $\left(X, T^{N}\right)$ is a WAP with $N$ minimal fixed points $x_{0}, \ldots, x_{N-1}$. For any $x \in X$ the restriction of $T^{N}$ to the $T^{N}$ orbit closure of $x$ is a point transitive WAP and so with a unique minimal set, necessarily one of the $x_{i}$ 's. Let $X_{i}=\{x \in$ $\left.X: x_{i} \in \overline{\mathcal{O}_{T^{N}}(x)}\right\}$ Clearly, $T\left(X_{i}\right)=X_{i+1}$ (addition $\bmod N$ ) and the $X_{i}$ 's are pairwise disjoint. Each is $T^{N}$ invariant. Let $u$ be a minimal element of $E\left(X, T^{N}\right)$. If $x \in X_{i}$ then $u(x)$ is a minimal element of the $T^{N}$ orbit closure of $x$ and so $u(x)=x_{i}$. That is, $u$ retracts $X_{i}$ to $x_{i}$. Because $\left(X, T^{N}\right)$ is WAP, $u$ is continuous and so each $X_{i}=u^{-1}\left(x_{i}\right)$ is closed.

Define $H: X_{0} \times\{0, \ldots, N-1\} \rightarrow X$ by $H(x, i)=T^{i}(x)$. This is continuous and surjective with inverse, $x \mapsto\left(T^{-i} x, i\right)$ for $x \in X_{i}$ and so $H$ is bijective. Furthermore, $H(x, i+1)=T(H(x, i))$ for $i<N-1$ and $H\left(T^{N}(x), 0\right)=$ $T^{N}(x)=T\left(T^{N-1}(x)\right)=T(H(x, N-1))$. Thus, $H$ is an isomorphism from the discrete suspension of height $N$ of $\left(X_{0}, T^{N}\right)$ onto $(X, T)$.

Recall that $(X, T)$ is minCT when there is a fixed point which is the unique minimal subset, i.e. the mincenter is a single point.

Lemma 1.45. Let $(X, T)$ be a nontrivial, metric minCT system with fixed point $e$ and let $\epsilon>0$. There exists an $\epsilon$-dense sequence of distinct points $\left\{e=x_{0}, \ldots, x_{N-1}\right\}$ in $X$ such that with $x_{N}=e,\left\{x_{0}, \ldots, x_{N}\right\}$ is an $\epsilon$ chain for $(X, T)$, i.e. $d\left(T\left(x_{i}\right), x_{i+1}\right)<\epsilon$ for $i=0, \ldots, N-1$.

Proof: Since $X$ is separable we can choose a finite or infinite sequence $\left\{a_{1}, a_{2}, \ldots\right\}$ of points of $X \backslash\{e\}$ with pairwise distinct orbits and such that the union of the orbits is dense in $X \backslash\{e\}$. Since this set is nonempty the sequence contains at least one point. Since $e$ is the only minimal point, $e \in \alpha_{T}(x) \cap \omega_{T}(x)$ 
for every $x \in X$. Now truncate so that the union of the orbits of the finite sequence $\left\{a_{1}, \ldots, a_{K}\right\}$ is $\epsilon / 2$ dense in $X \backslash\{e\}$. For each $a_{i}$ we can choose a finite piece of the orbit $\left\{y_{0, i}, \ldots, y_{K_{i}+1, i}\right\}$ which begins and ends $\epsilon / 2$ close to $e$ and which is $\epsilon / 2$ dense in the orbit of $a_{i}$. We concatenate to obtain the sequence $\left\{x_{1}, \ldots, x_{N-1}\right\}$. Then let $x_{0}=e$.

On the one-point compactification $\mathbb{Z}^{*}$ with $e$ the point at infinity and $T(t)=$ $t+1$, define the ultra-metric $d$ by

$$
d(i, j)=\left\{\begin{array}{cl}
0 & \text { if } i=j \\
\max (1 /(|i|+1), 1 /(|j|+1)) & \text { if } i \neq j
\end{array}\right.
$$

where $1 /(|i|+1)=0$ if $i=e$. If $(X, T)=\left(\mathbb{Z}^{*}, T\right)$ then with $K>1 / \epsilon$ and $N=2 K+2$ we can use the sequence $\{e,-K,-K+1, \ldots, K\}$.

When $(X, T)$ is a nontrivial metric minCT system we define a preparation for $(X, T)$ to be a choice for each $i=0,1, \ldots$ of a sequence $\{e=$ $\left.x_{0}^{i}, \ldots, x_{N_{i}-1}^{i}\right\}$ which is an $2^{-i}$ dense sequence of distinct elements of $X$ so that $\left\{e=x_{0}^{i}, \ldots, x_{N_{i}-1}^{i}, e\right\}$ is a $2^{-i}$ chain. For $i=0$ we let $N_{0}=1$ so that with $i=0$ the sequence is $\{e\}$.

An ultrametric minCT system is a minCT system $(X, T)$ with $d \leq 1$ a compatible ultra-metric on $X$ (and so $X$ is zero-dimensional).

As we will dealing with different metrics at the same time we will use for $\epsilon>0$

$$
V_{d, \epsilon}=\{(a, b): d(a, b)<\epsilon\}
$$

so that $V_{d, \epsilon}(a)$ is the $\epsilon$ ball centered at $a$. For an ultrametric $V_{d, \epsilon}$ is a clopen equivalence relation and so each ball $V_{d, \epsilon}(a)$ is a clopen set.

Let $\left(X_{1}, T_{1}\right),\left(X_{2}, T_{2}\right)$ be nontrivial ultrametric minCT systems with fixed points $e_{1}, e_{2}$. Assume that $\left(X_{2}, T_{2}\right)$ is given a preparation. The ultrametrics on $X_{1}$ and $X_{2}$ are labeled $d_{1}$ and $d_{2}$, respectively. From the product $X_{1} \times X_{2}$ ) we have the projections $\pi_{1}$ and $\pi_{2}$ to $X_{1}$ and $X_{2}$, respectively.

Fix $1>\epsilon>0$. On $X_{1} \times X_{2}$ we will use the ultrametric $d=\max \left(\pi_{1}^{*} d_{1}, \epsilon \pi_{2}^{*} d_{2}\right)$, i.e. $d\left(\left(x_{1}, x_{2}\right),\left(y_{1}, y_{2}\right)\right)=\max \left(d_{1}\left(x_{1}, y_{1}\right), \epsilon d_{2}\left(x_{2}, y_{2}\right)\right.$. so that $\pi_{1}$ has Lipschitz constant 1 and for any $\delta>0$

$$
V_{d, \delta}\left(e_{1}, e_{2}\right) \quad \subset \quad \pi_{1}^{-1}\left(V_{d_{1}, \delta}\left(e_{1}\right)\right)
$$

with equality if $\delta \geq \epsilon$.

We will define the $\epsilon$ spin of $\left(X_{2}, T_{2}\right)$ into $\left(X_{1}, T_{1}\right)$ to be the ultrametric system $(X, T)$ where $X$ is the closed subset of $X_{1} \times X_{2}$ and the homeomorphism $T$ on $X$ are described below. 
In $X_{1}$ we define the sequence of pairwise disjoint clopen sets: $A_{0}=X_{1} \backslash$ $V_{d_{1}, \epsilon}\left(e_{1}\right)$ and for $i=1,2, \ldots, A_{i}=V_{d_{1}, \epsilon 2^{-i+1}}\left(e_{1}\right) \backslash V_{d_{1}, \epsilon 2^{-i}}\left(e_{1}\right)$. So $X_{1}=\left\{e_{1}\right\} \cup$ $\bigcup_{i=0}^{\infty} A_{i}$. Now let

$$
\begin{aligned}
X= & \left(\left\{e_{1}\right\} \times X_{2}\right) \cup\left(\bigcup_{i=0}^{\infty} A_{i} \times\left\{x_{0}^{i}, \ldots, x_{N_{i}-1}^{i}\right\}\right), \\
T(x, y)= & \left\{\begin{array}{lr}
\left(e_{1}, T_{2}(y)\right) & \text { when } x=e_{1}, \\
\left(x, x_{k+1}^{i}\right) & \text { when } x \in A_{i}, \quad y=x_{k}^{i} \\
\left(T_{1}(x), e_{2}\right) & \text { when } x \in A_{i}, \quad y=x_{N_{i}-1}^{i} .
\end{array} \text { with } k<N_{i}-1,\right.
\end{aligned}
$$

It is obvious that $X$ is a closed subset of $X_{1} \times X_{2}$ and easy to check that $T$ is invertible with $T^{-1}\left(x, e_{2}\right)=\left(T_{1}^{-1}(x), x_{N_{i}-1}^{i}\right)$ when $T_{1}^{-1}(x) \in A_{i}$. Continuity of $T$ is clear on each $A_{i} \times\left\{x_{0}^{i}, \ldots, x_{N_{i}-1}^{i}\right\}$, because each $A_{i} \times\left\{x_{j}^{i}\right\}$ is a clopen subset of $X$. Continuity at the points of $\left\{e_{1}\right\} \times X_{2}$ follows from the following estimate.

Lemma 1.46. (a) Let $\delta \geq \epsilon$. If $(x, y) \in X$ with $x \in V_{d_{1}, \delta}\left(e_{1}\right) \cap T_{1}^{-1}\left(V_{d_{1}, \delta}\left(e_{1}\right)\right)$ then $(x, y) \in V_{d, \delta}\left(e_{1}, e_{2}\right) \cap T^{-1}\left(V_{d, \delta}\left(e_{1}, e_{2}\right)\right)$.

(b) For $i \geq 1$, let $\delta$ with $2^{-i}>\delta>0$ be a $2^{-i}$ modulus of uniform continuity for $T_{2}$ on $X_{2}$. For $(x, y) \in X, \tilde{y} \in X_{2}$

$$
\begin{array}{r}
x \in V_{d_{1}, \epsilon 2^{-i+1}}\left(e_{1}\right) \cap T_{1}^{-1}\left(V_{d_{1}, 2^{-i+1}}\left(e_{1}\right)\right), y \in V_{d_{2}, \delta}(\tilde{y}) \\
\Longrightarrow T(x, y) \in V_{d, 2^{-i+1}}\left(e_{1}, T_{2}(\tilde{y})\right) .
\end{array}
$$

Proof: (a) follows from (1.14) applied to $x$ and to $T_{1}(x)$.

(b) If $x=e_{1}$ then $T(x, y)=\left(e_{1}, T_{2}(y)\right)$ and $d\left(T_{2}(y), T_{2}(\tilde{y})\right) \leq 2^{-i}$ if $y \in$ $V_{d_{2}, \delta}(\tilde{y})$.

If $x \in V_{d_{1}, \epsilon 2^{-i+1}}\left(e_{1}\right) \backslash\left\{e_{1}\right\}$ then $X_{2}$ coordinates of the fiber in $X$ over $x$ is a $2^{-i}$ chain for $T_{2}$ and so if $y \in V_{d_{2}, \delta}(\tilde{y})$ then the second coordinate of $T(x, y)$ is within $2^{-i+1}=2^{-i}+2^{-i}$ of $T_{2}(\tilde{y})$.

In particular, if $j \geq i$ and $(x, y)=\left(x, x_{N_{j}-1}^{j}\right)$ with $x \in A_{j}$ then $d_{2}(y, \tilde{y})=$ $d_{2}\left(x_{N_{j}-1}^{j}, \tilde{y}\right)<\delta$ and by definition of the chains in a preparation, $d_{2}\left(T_{2}\left(x_{N_{j}-1}^{j}\right), e_{2}\right)$ $<2^{-j}$. Since $T(x, y)=\left(T_{1}(x), e_{2}\right)$ we again have that the second coordinate of $T(x, y)$ is within $2^{-i+1}=2^{-i}+2^{-i}$ of $T_{2}(\tilde{y})$.

So we obtain the ultrametric system $(X, T)$. 
From (1.14) it follows that the restriction

$$
\begin{aligned}
\pi_{1}: X \backslash V_{d, \epsilon}\left(e_{1}, e_{2}\right) & =\left(\pi_{1}\right)^{-1}\left(X_{1} \backslash V_{d_{1}, \epsilon}\left(e_{1}\right)\right) \rightarrow X_{1} \backslash V_{d_{1}, \epsilon}\left(e_{1}\right) \text { is bijective, } \\
& \text { and on it } \pi_{1} \circ T=T_{1} \circ \pi_{1}, \\
x \in X_{1} \backslash V_{\epsilon}\left(e_{1}\right) & \Longrightarrow\left(\pi_{1}\right)^{-1}(x)=\left\{\left(x, e_{2}\right)\right\}, \quad T\left(x, e_{2}\right)=\left(T_{1}(x), e_{2}\right) .
\end{aligned}
$$

On the rest of the space the map $\pi_{1}: X \rightarrow X_{1}$ does not define an action map, but we obviously have for $x \in X_{1} \backslash\left\{e_{1}\right\}$ :

$$
\begin{aligned}
\pi_{1}^{-1}\left(\left\{T_{1}^{i}(x): i=0,1, \ldots\right\}\right) & =\left\{T^{i}\left(x, e_{2}\right): i=0,1, \ldots\right\}, \\
\pi_{1}^{-1}\left(\left\{T_{1}^{-i}(x): i=1,2, \ldots\right\}\right) & =\left\{T^{-i}\left(x, e_{2}\right): i=1,2, \ldots\right\} .
\end{aligned}
$$

Proposition 1.47. If $x \in X_{1} \backslash\left\{e_{1}\right\}$, then

$$
\pi_{1}^{-1}\left(\omega T_{1}(x)\right)=\omega T\left(x, e_{2}\right), \quad \pi_{1}^{-1}\left(\alpha T_{1}(x)\right)=\alpha T\left(x, e_{2}\right) .
$$

If $A$ is a $T_{1}$ invariant subset of $X_{1}$ then $\pi_{1}^{-1}(A)$ is a $T$ invariant subset of $X$.

If $B$ is a $T$ invariant subset of $X$ then $\pi_{1}(B)$ is a $T_{1}$ invariant subset of $X_{1}$.

Proof: The equations (1.18) clearly imply that $\pi_{1}$ maps the limit point set $\omega T\left(x, e_{2}\right)$ onto $\omega T_{1}(x)$. Then they imply that if $z \in X_{1} \backslash\left\{e_{1}\right\}$ then all the points of $\pi_{1}^{-1}(z)$ lie in the same orbit. Finally, for $z \in V_{d_{1}, 2^{-i}}\left(e_{1}\right) \backslash\left\{e_{1}\right\}$ the set $\pi_{2}\left(\pi_{1}^{-1}(z)\right)$ is $\epsilon 2^{-i}$ dense in $X_{2}$. This proves the result for $\omega T(x)$ and the result for $\alpha T(x)$ is similar.

The invariant set results are obvious from (1.18).

Corollary 1.48. $(X, T)$ is a minCT system.

If $\left(X_{1}, T_{1}\right)$ and $\left(X_{2}, T_{2}\right)$ are CT systems then $(X, T)$ is a CT system. Furthermore, if the Birkhoff center sequences for $\left(X_{1}, T_{1}\right)$ and $\left(X_{2}, T_{2}\right)$ stabilize at ordinals $\omega_{1}$ and $\omega_{2}$ respectively, then the Birkhoff center sequence for $(X, T)$ stabilizes at $\omega_{1}+\omega_{2}$.

Proof: If $M$ is a minimal subset of $X$ then by Proposition $1.47 \pi_{1}$ is a minimal subset of $X_{1}$ and so $M \subset\left\{e_{1}\right\} \times X_{2}$ where $T$ is isomorphic to $T_{2}$ and so the $M=\left\{\left(e_{1}, e_{2}\right)\right\}$.

Now assume $\left(X_{1}, T_{1}\right)$ and $\left(X_{2}, T_{2}\right)$ are CT systems. If $(x, y)$ is a recurrent point for $T$ then $x_{1}$ is a recurrent point for $X_{1}$ by (1.19). Hence, $x=e_{1}$ and $y$ is a recurrent point for $T_{2}$. So $y=e_{2}$. Hence, $(X, T)$ is CT. If $A$ is a closed $T_{1}$ invariant subset of $X_{1}$ then the limit point set $R_{T}\left(\pi_{1}^{-1}(A)\right)$ is the limit point set $\pi_{1}^{-1}\left(R_{T_{1}}(A)\right)$. So exactly at $\omega_{1}$ the Birkhoff center sequence for $X$ arrives at $\left\{e_{1}\right\} \times X_{2}$. It then stabilizes at $\left(e_{1}, e_{2}\right)$ after $\omega_{2}$ more steps. 
Notice that by replacing the metric $d$ on $X$ by the equivalent metric

$$
\min \left(1, \max _{n=0}^{\infty} 2^{-n}\left(T^{n}\right)^{*} d\right)
$$

we can assume that the metric is bounded by 1 and that $T$ has Lipschitz constant at most 2. The new metric is an ultrametric if $d$ was.

Let $\left\{\left(X_{n}, T_{n}\right): n=1,2, \ldots\right\}$ be a sequence of ultrametric minCT systems with ultrametric $d_{n} \leq 1$ on $X_{n}$ and with each $T_{n}$ having Lipschitz constant at most 2. Assume that for $n>1$ each $\left(X_{n}, T_{n}\right)$ is given a preparation. Let $\left(Z_{1}, U_{1}\right)=\left(X_{1}, T_{1}\right)$, let $\left(Z_{2}, U_{2}\right)$ be the $2^{-1}$ spin of $\left(X_{2}, T_{2}\right)$ into $\left(Z_{1}, U_{1}\right)$ with $\xi_{2}: Z_{2} \rightarrow Z_{1}$ be the first coordinate projection. Thus, $Z_{2} \subset X_{1} \times X_{2}$. Inductively, let $\left(Z_{n+1}, U_{n+1}\right)$ be the $2^{-n}$ spin of $\left(X_{n+1}, T_{n+1}\right)$ into $\left(Z_{n}, U_{n}\right)$ which we can regard as a subset of the product $\Pi_{n}=X_{1} \times \cdots \times X_{n} \times X_{n+1}$ equipped with the ultrametric $\max \left(\pi_{1}^{*} d_{1}, 2^{-1} \pi_{2}^{*} d_{2}, \ldots, 2^{-n-1} \pi_{n+1}^{*} d_{n+1}\right)$. Let $\xi_{n+1}: Z_{n+1} \rightarrow Z_{n}$ be the restriction of the coordinate projection from $\Pi_{n+1} \rightarrow$ $\Pi_{n}$ which has Lipschitz constant 1 . Note again that the $\xi_{n}$ 's are not action maps, but by (1.17) the restriction $\xi_{n}:\left(\xi_{n}\right)^{-1}\left(Z_{n} \backslash V_{2^{-n}}\left(e_{1}, \cdots, e_{n}\right)\right) \rightarrow Z_{n} \backslash$ $V_{2^{-n}}\left(e_{1}, \ldots, e_{n}\right)$ is injective and on it $\xi_{n} \circ U_{n+1}=U_{n} \circ \xi_{n}$.

Let $Z_{\infty}$ denote the inverse limit, regarded as a closed subset of $\Pi_{\infty}=\prod_{i=1}^{\infty} X_{i}$ equipped with the ultrametric $\max \left\{2^{-i+1} \pi_{i}^{*} d_{i}: i=1,2, \ldots\right\}$ which yields the product topology. The space $Z_{\infty}$ consists of the points $z$ such that $\xi_{n}(z)=$ $\left(z_{1}, \ldots, z_{n}\right) \in Z_{n}$ for $n=1,2, \ldots$ We let $e \in Z_{\infty}$ denote the point $\left(e_{1}, e_{2}, \ldots\right)$.

Assume that $z \in Z_{\infty}$ with $z \neq e$ and let $n$ be the smallest value such that $x=\xi_{n}(z) \neq \xi_{n}(e)=\left(e_{1}, \ldots, e_{n}\right)$. Let $\delta=\frac{1}{2} d\left(x,\left(e_{1}, \ldots, e_{n}\right)\right)$. Let $i>n$ be such that $2^{-i} \leq \delta$. Since the projections have Lipschitz constant 1 , is disjoint from $V_{\delta}\left(e_{1}, \ldots, e_{n+k}\right)$ for every positive integer $k$. Once $n+k \geq i$ it follows that $\left(\xi_{n+k-1} \circ \cdots \circ \xi_{n}\right)^{-1}\left(V_{\delta}(x)\right)$ is disjoint from $V_{2^{-n-k}}\left(e_{1}, \ldots, e_{n+k}\right)$. By (1.17) if $\tilde{x} \in Z_{n+k} \backslash V_{2^{-n-k}}\left(e_{1}, \ldots, e_{n+k}\right)$ and $\tilde{z}=\left(\tilde{x}, e_{n+k+1}, e_{n+k+2}, \ldots\right) \in Z_{\infty}$ then $\xi_{n+k}^{-1}(\tilde{x})=\{\tilde{z}\}$ and by (1.17) $U(\tilde{z})$ is unambiguously defined by $U(\tilde{z})=$ $\left(U_{n+k}(\tilde{x}), e_{n+k+1}, e_{n+k+2}, \ldots\right)$. Since each $U_{n+k}$ has Lipschitz constant at most 2 , it follows that on each $Z_{\infty} \backslash \xi_{n+k}^{-1}\left(V_{2^{-n-k}}\left(e_{1}, \ldots, e_{n+k}\right)\right) U$ has Lipschitz constant at most 2. Finally,

$$
d(U(\tilde{z}), e)=d\left(U_{n+k}(\tilde{x}),\left(e_{1}, \ldots, e_{n+k}\right)\right) \leq 2 d\left(\tilde{x},\left(e_{1}, \ldots, e_{n+k}\right)\right)=2 d(\tilde{z}, e)
$$

shows that $U$ has Lipschitz constant at most 2 on all of $Z_{\infty}$.

Finally, with essentially the same proof as that of Corollary 1.48 we have

Corollary 1.49. $\left(Z_{\infty}, U\right)$ is a minCT system.

If each $\left(X_{n}, T_{n}\right)$ is a CT system then $\left(Z_{\infty}, U\right)$ is a CT system. Furthermore, if the Birkhoff center sequences for $\left(X_{n}, T_{n}\right)$ stabilize at the ordinals $\omega_{n}$, then the Birkhoff center sequence for $\left(Z_{\infty}, U\right)$ stabilizes at $\operatorname{Lim}_{n \rightarrow \infty} \omega_{1}+\omega_{2}+\cdots+\omega_{n}$. 


\section{LABELS AND THEIR DYNAMICS}

\subsection{The space of labels.}

Let $\mathbb{Z}, \mathbb{Z}_{+}, \mathbb{N}$ denote the sets of integers, of non-negative integers and of positive integers, respectively. Let $\mathbb{Z}_{+\infty}=\mathbb{Z}_{+} \cup\{\infty\}=\mathbb{N} \cup\{0, \infty\}$. On the vector space $\mathbb{R}^{\mathbb{N}}$ we will use the lattice structure, with $x \geq y, x \leq y, x \vee$ $y, x \wedge y$, the pointwise relations and the pointwise operations of maximum and minimum for vectors $x, y \in \mathbb{R}^{\mathbb{N}}$. As usual $x>y$ means $x \geq y$ and $x \neq y$ so that the inequality is strict for at least one coordinate. The support of a vector $x \in \mathbb{R}^{\mathbb{N}}$, denoted supp $x$, is $\left\{\ell: x_{\ell} \neq 0\right\}$. We identify the number $n \in \mathbb{N}$ with the function in $\mathbb{Z}_{+}^{\mathbb{N}}$ which is constant with value $n$.

We will call $\mathbf{m} \in \mathbb{Z}^{\mathbb{N}}$ an $\mathbb{N}$-vector when it is non-negative and has finite support, that is, when $\mathbf{m} \geq 0$ and supp $\mathbf{m}=\left\{\ell: \mathbf{m}_{\ell}>0\right\}$ is finite. We call \#supp $\mathbf{m}$ the size of $\mathbf{m}$ and call $|\mathbf{m}|=\Sigma_{\ell} \mathbf{m}_{\ell}$ the norm of $\mathbf{m}$.

If $S \subset \mathbb{N}$ we let $\chi(S)$ be the characteristic function of $S$ with $\chi(\ell)=\chi(\{\ell\})$. Thus, $\chi(S)=\Sigma_{\ell \in S} \chi(\ell)$ is an $\mathbb{N}$-vector when $S$ is a finite set.

We denote by $F I N(\mathbb{N})$ the discrete abelian monoid of all $\mathbb{N}$-vectors with vector addition and identity $\mathbf{0}$. It is also a lattice via the pointwise order relations described above.

For an $\mathbb{N}$-vector $\mathbf{m}$ and $L$ a nonempty subset of $\mathbb{N}$ we define $\mathbf{m} \wedge L$ to be the $\mathbb{N}$-vector with

$$
(\mathbf{m} \wedge L)_{\ell}=\left\{\begin{aligned}
\mathbf{m}_{\ell} & \text { for } \quad \ell \in L, \\
0 & \text { for } \quad \ell \notin L .
\end{aligned}\right.
$$

In particular, for a positive integer $\ell^{*}, \mathbf{m} \wedge\left[1, \ell^{*}\right]$ is given by

$$
\left(\mathbf{m} \wedge\left[1, \ell^{*}\right]\right)_{\ell}=\left\{\begin{aligned}
\mathbf{m}_{\ell} & \text { for } \ell \leq \ell^{*} \\
0 & \text { for } \ell>\ell^{*}
\end{aligned}\right.
$$

Definition 2.1. A set $\mathcal{M}$ of $\mathbb{N}$-vectors is called a label when $\mathbf{0} \leq \mathbf{m}^{1} \leq \mathbf{m}$ and $\mathbf{m} \in \mathcal{M}$ imply $\mathbf{m}^{1} \in \mathcal{M}$. We call this the Heredity Condition.

We use the term "label" because we will be using them to label certain associated subshifts.

For example, $0=\{\mathbf{0}\}$ and $\emptyset$ are labels. 
Definition 2.2. (a) Let $\operatorname{Supp} \mathcal{M}=\{\operatorname{supp} \mathbf{m}: \mathbf{m} \in \mathcal{M}\}$, the set of supports of members of $\mathcal{M}$. Thus, $\operatorname{Supp} 0=\{\emptyset\}$ and $\operatorname{Supp} \emptyset=\emptyset$.

(b) For $S \subset \mathbb{Z}_{+\infty}^{\mathbb{N}}$, Let $\langle S\rangle=\{\mathbf{m} \in F I N(\mathbb{N}): \mathbf{m} \leq \nu$ for some $\rho \in S\}$. We call $\langle S\rangle$ the label generated by $S$. In particular, if $\rho: \mathbb{N} \rightarrow \mathbb{Z}_{+\infty}$ we will write $\langle\rho\rangle$ for $\langle S\rangle$ when $S=\{\rho\}$ so that $\langle\rho\rangle=\{\mathbf{m} \in F I N(\mathbb{N})$ : $\mathbf{m} \leq \rho\}$.

(c) We will say that Supp $\mathcal{M} f$-contains a set $L \subset \mathbb{N}$ when every finite subset of $L$ is a member of Supp $\mathcal{M}$. That is, $\mathcal{P}_{f} L \subset S u p p \mathcal{M}$ where $\mathcal{P}_{f} L$ is the set of finite subsets of $L$. Equivalently, $\mathcal{M} \supset\langle\chi(L)\rangle$.

(d) For $N \in \mathbb{Z}_{+}$, let $\mathcal{B}_{N}=\langle(N-1) \chi([1, N])\rangle$. That is, $\mathbf{m} \in \mathcal{B}_{N}$ iff $\mathbf{m}<N$, i.e. $\mathbf{m}_{\ell}<N$ for all $\ell \in \mathbb{N}$, and and supp $\mathbf{m} \subset[1, N]$.

In particular, $\mathcal{B}_{0}=\emptyset$ and $\mathcal{B}_{1}=0$. Thus, $\left\{\mathcal{B}_{N}\right\}$ is an increasing sequence of finite labels with union $F I N(\mathbb{N})$, the maximum label.

Definition 2.3. Let $\mathcal{M}$ be a label.

(a) $\mathcal{M}$ is bounded if there exists $\rho \in \mathbb{Z}_{+}^{\mathbb{N}}$ such that $0 \leq \mathbf{m} \leq \rho$ for all $\mathbf{m} \in \mathcal{M}$ i.e. $\mathcal{M} \subset\langle\rho\rangle$. Note that $\rho_{\ell}<\infty$ for all $\ell \in \mathbb{N}$. We call this the Bound Condition.

(b) $\mathcal{M}$ is of finite type if it there does not exist an infinite increasing sequence in $\mathcal{M}$, or equivalently, any infinite nondecreasing sequence in $\mathcal{M}$ is eventually constant. We call this the Finite Chain Condition.

(c) $\mathcal{M}$ is size bounded if there exists $n \in \mathbb{N}$ such that $\operatorname{size}(\mathbf{m}) \leq n$ for all $\mathbf{m} \in \mathcal{M}$. We call this the Size Bound Condition

Clearly, a finite label is of finite type.

For example, $\emptyset$ and $0=\{\mathbf{0}\}$ are finite labels. $\mathcal{M} \neq \emptyset$ iff $\mathbf{0} \in \mathcal{M}$. We call $\mathcal{M}$ a positive label when it is neither $\emptyset$ nor 0 .

For a label $\mathcal{M}$ we let $[[\mathcal{M}]]$ denote the set of labels which are contained in $\mathcal{M}$. Clearly, if $\mathcal{M}$ is bounded, of finite type, size bounded or finite then all of the labels in $[[\mathcal{M}]]$ satisfy the corresponding property.

Define the roof $\rho(\mathcal{M}): \mathbb{N} \rightarrow \mathbb{Z}_{+\infty}$ of a label $\mathcal{M}$ by

$$
\rho(\mathcal{M})_{\ell}=\sup _{\mathbf{m} \in \mathcal{M}}\left\{\mathbf{m}_{\ell}\right\}=\sup \left\{r \in \mathbb{Z}_{+}: r \chi(\ell) \in \mathcal{M}\right\} \leq \infty
$$

Thus, $\mathcal{M}$ is bounded iff $\rho(\mathcal{M})_{\ell}<\infty$ for all $\ell$ in which case the roof is the minimum of the functions $\rho \in \mathbb{Z}_{+}^{\mathbb{N}}$ which bound the elements of $\mathcal{M}$. Clearly, $\rho(0)=0$. We will use that convention that $\sup \emptyset=0$ so that $\rho(\emptyset)=0$ as well.

Lemma 2.4. If a label is of finite type then it is bounded. If a label is bounded and size bounded then it is of finite type.

Proof: If $\rho(\mathcal{M})_{\ell}=\infty$ then $\{i \chi(\ell): i \in \mathbb{N}\}$ is an infinite increasing sequence in $\mathcal{M}$. 
Now assume that $\mathcal{M}$ is bounded by $\rho \in \mathbb{Z}_{+}^{\mathbb{N}}$. If $\mathbf{m}^{1}<\mathbf{m}^{2}<\cdots$ is an infinite sequence of $\mathbb{N}$-vectors then at each step either some entry increases or the size increases. Since the entries in $\mathcal{M}$ are bounded by $\rho$ and the size is assumed bounded the sequence must eventually leave $\mathcal{M}$. Hence, the Finite Chain Condition holds.

For a label $\mathcal{M}$ and $\ell^{*} \in \mathbb{N}$ we define

$$
\mathcal{M} \wedge\left[1, \ell^{*}\right]=\left\{\begin{array}{c}
\emptyset \\
\left\{\mathbf{m} \wedge\left[1, \ell^{*}\right]: \mathbf{m} \in \mathcal{M}\right\} \quad \text { when } \ell^{*}=0,
\end{array}\right.
$$

Thus, for $\ell^{*} \in \mathbb{N}, \mathcal{M} \wedge\left[1, \ell^{*}\right]=\left\{\mathbf{m} \in \mathcal{M}: \operatorname{supp} \mathbf{m} \subset\left[1, \ell^{*}\right]\right\}$.

For a label $\mathcal{M}$ and an $\mathbb{N}$-vector $\mathbf{r}$ we define

$$
\mathcal{M}-\mathbf{r}=\{\mathbf{w} \in F I N(\mathbb{N}): \mathbf{w}+\mathbf{r} \in \mathcal{M}\} .
$$

Thus, $\mathcal{M}-\mathbf{r}$ is the set of all non-negative vectors of the form $\mathbf{m}-\mathbf{r}$ for $\mathbf{m} \in \mathcal{M}$. Clearly, $(\mathcal{M}-\mathbf{r})-\mathbf{s}=\mathcal{M}-(\mathbf{r}+\mathbf{s})=(\mathcal{M}-\mathbf{s})-\mathbf{r}$ (and so we can omit the parentheses) since each is the set of $\mathbb{N}$-vectors $\mathbf{w}$ such that $\mathbf{w}+\mathbf{r}+\mathbf{s} \in \mathcal{M}$.

Let $\max \mathcal{M}$ denote the set of $\mathbb{N}$-vectors which are maximal in $\mathcal{M}$. That is, $\mathbf{n} \in \max \mathcal{M}$ if

$$
\mathbf{m} \geq \mathbf{n} \text { and } \mathbf{m} \in \mathcal{M} \quad \Longleftrightarrow \quad \mathbf{m}=\mathbf{n} .
$$

Proposition 2.5. Let $\mathcal{M}$ be a label and let $\mathbf{r}>\mathbf{0}$ be an $\mathbb{N}$-vector.

(a) If $\mathcal{M}$ is of finite type then for every $\mathbf{m} \in \mathcal{M}$ there exists $\mathbf{n} \in \max \mathcal{M}$ such that $\mathbf{m} \leq \mathbf{n}$. Hence, $\mathcal{M}=\langle\max \mathcal{M}\rangle$. Thus, a label $\mathcal{M}$ of finite type is determined by $\max \mathcal{M}$. Also, every $A \in S u p p \mathcal{M}$ is contained in some $B \in$ Supp $\mathcal{M}$ which is maximal with respect to inclusion in Supp $\mathcal{M}$.

(b) If $\mathcal{M} f$-contains some infinite set $L \subset \mathbb{N}$ then $\mathcal{M}$ is not of finite type. Conversely, if $\mathcal{M}$ is bounded but not of finite type then it f-contains some infinite set L. Thus, a bounded label is of finite type iff it does not $f$-contain an infinite set.

(c) If $\mathcal{M}$ is bounded and $\ell^{*} \in \mathbb{N}$ then $\mathcal{M} \wedge\left[1, \ell^{*}\right]$ is a finite label.

(d) $\mathcal{M}-\mathbf{r}$ is a label contained in $\mathcal{M}$ with $\max \mathcal{M} \subset \mathcal{M} \backslash(\mathcal{M}-\mathbf{r})$. If $\mathcal{M}$ is nonempty and bounded then $\mathcal{M}-\mathbf{r}$ is a proper subset of $\mathcal{M}$.

(e) $\mathcal{M}-\mathbf{r} \neq \emptyset$ iff $\mathbf{r} \in \mathcal{M}$.

(f) If $\Phi$ is a set of labels then $\bigcup \Phi$ and $\bigcap \Phi$ are labels.

(g) If $\Phi$ is a finite set of labels then $\bigcup \Phi$ is of finite type iff all of the labels in $\Phi$ are.

Proof: (a): If $\mathbf{m}$ is not maximal then there exists $\mathbf{m}_{1} \in \mathcal{M}$ with $\mathbf{m}_{1}>\mathbf{m}$. Continue if $\mathbf{m}_{1}$ is not maximal. This sequence can continue only finitely many 
steps by the Finite Chain Condition. It terminates at a maximal vector $\mathbf{n}$. Similarly, if $A \in S u p p \mathcal{M}$ is not contained in a maximal element then there is an increasing sequence in $\left\{A_{0}, A_{1}, \ldots\right\}$ in Supp $\mathcal{M}$ with $A=A_{0}$. Then $\mathbf{m}^{k}=\chi\left(A_{k}\right)$ is a strictly increasing sequence in $\mathcal{M}$.

(b) If $\mathcal{M}$ f-contains $L=\left\{\ell_{1}, \ell_{2}, \ldots\right\}$ then $\mathbf{n}^{k}=\sum_{i=1}^{k} \chi\left(\ell_{i}\right)$ is a strictly increasing infinite sequence in $\mathcal{M}$ and so $\mathcal{M}$ is not of finite type. Conversely, that if $\mathbf{n}^{k} \in \mathcal{M}$ is an infinite increasing sequence in $\mathcal{M}$ then $\mathcal{M}$ f-contains the union $L$ of the increasing sequence $\left\{\operatorname{supp} \mathbf{n}^{k}\right\}$ of finite sets. If $\mathcal{M}$ is bounded then $L$ must be an infinite set.

(c): For any $\mu \in \mathbb{Z}_{+}^{\mathbb{N}}$ and $\ell^{*}>0$, if $N=\max _{\ell=1}^{\ell^{*}}\left\{1+\mu_{\ell}\right\}$, then $\mathcal{M} \wedge\left[1, \ell^{*}\right] \subset$ $\mathcal{B}_{N}$.

(d) $\mathcal{M}-\mathbf{r}$ satisfies the Heredity Condition and so is a label contained in $\mathcal{M}$. If $\mathbf{m} \in \mathcal{M}-\mathbf{r}$ then $\mathbf{m}+\mathbf{r}$ is an element of $\mathcal{M}$ with $\mathbf{m}+\mathbf{r}>\mathbf{m}$ since $\mathbf{r}>\mathbf{0}$. Hence, $\mathbf{m}$ is not a maximal element of $\mathcal{M}$.

Now assume $\mathcal{M}$ is nonempty and bounded. Then $\mathbf{0}=0 \mathbf{r} \in \mathcal{M}$. If $\mathbf{r}_{\ell}>0$ then for $n \in \mathbb{N}$ such that $n>\rho(\mathcal{M})_{\ell} / \mathbf{r}_{\ell}, n \mathbf{r} \notin \mathcal{M}$. So there is a maximum $N \geq 0$ such that $N \mathbf{r} \in \mathcal{M}$. It follows that $N \mathbf{r} \in \mathcal{M} \backslash(\mathcal{M}-\mathbf{r})$.

(e) If $\mathbf{r} \in \mathcal{M}$ then $0 \in \mathcal{M}-\mathbf{r}$. If $\mathbf{m} \in \mathcal{M}-\mathbf{r}$ then $\mathbf{m}+\mathbf{r} \in \mathcal{M}$ and so $\mathbf{m}+\mathbf{r} \geq \mathbf{r}$ implies $\mathbf{r} \in \mathcal{M}$.

(f) : Obvious.

(g) If $\Phi$ is finite collection of labels of finite type and $\left\{\mathbf{m}^{i}\right\}$ is a strictly increasing sequence of $\mathbb{N}$-vectors then it can remain in each member of $\Phi$ for only finitely many terms. As $\Phi$ is finite, the sequence must eventually leave $\bigcup \Phi$. Hence, the union satisfies the Finite Chain Condition.

If the union is of finite type then each member of $\Phi$ is of finite type by (b).

Example 2.6. A label $\mathcal{M}$ which is generated by max $\mathcal{M}$ need not be of finite type.

(a) If $\mathcal{M}=\left\langle\left\{2 \chi(k+1)+\sum_{\ell=1}^{k} \chi(\ell): k \in \mathbb{N}\right\}\right\rangle$ then $\mathcal{M}$ is not of finite type although every $\mathbf{m} \in \mathcal{M}$ is bounded by an element of $\max \mathcal{M}$.

(b) Let $\left.\mathcal{M}=\left\langle\left\{\chi(2 k+1)+\Sigma_{\ell=1}^{k} \chi(2 \ell): k \in \mathbb{N}\right\}\right\}\right\rangle$. Clearly $\mathcal{M}$ f-contains $L$ the infinite set of even numbers, but every $A \in S$ upp $\mathcal{M}$ is contained in some $B \in \operatorname{Supp} \mathcal{M}$ which is maximal with respect to inclusion in Supp $\mathcal{M}$.

We denote by $\mathcal{L} \mathcal{A B}$ the space of labels. On $\mathcal{L} \mathcal{A B}$ we define an ultrametric by

$$
d\left(\mathcal{M}_{1}, \mathcal{M}_{2}\right)=\inf \left\{2^{-N}: N \in \mathbb{Z}_{+} \text {and } \mathcal{M}_{1} \cap \mathcal{B}_{N}=\mathcal{M}_{2} \cap \mathcal{B}_{N}\right\} .
$$

Notice that since $\mathcal{B}_{0}=\emptyset, \mathcal{M}_{1} \cap \mathcal{B}_{0}=\mathcal{M}_{2} \cap \mathcal{B}_{0}$ is always true. 
Lemma 2.7. (a) $d\left(\mathcal{M}_{1}, \mathcal{M}_{2}\right)=0$ iff $\mathcal{M}_{1}=\mathcal{M}_{2}$.

(b) The label $\emptyset$ is an isolated point of $\mathcal{L} \mathcal{A B}$ with $d(\emptyset, \mathcal{M})=1$ for all $\mathcal{M} \neq \emptyset$.

(c) If $\mathcal{N}_{1} \subset \mathcal{N}$ are finite labels and $\mathbf{m} \in F I N(\mathbb{N})$ then each of the following is a clopen subset of $\mathcal{L} \mathcal{A B}$ :

$$
\begin{gathered}
\left\{\mathcal{M}: \mathcal{M} \cap \mathcal{N}=\mathcal{N}_{1}\right\}, \quad\{\mathcal{M}: \mathcal{M} \cap \mathcal{N}=0\}, \quad\{\mathcal{M}: \mathcal{N} \subset \mathcal{M}\}, \\
\{\mathcal{N}: \mathbf{m} \in \mathcal{M}\}, \quad\{\mathcal{M}: \mathbf{m} \notin \mathcal{M}\} .
\end{gathered}
$$

Furthermore, the set $\left\{\left(\mathcal{M}, \mathcal{M}_{1}\right): \mathcal{M} \cap \mathcal{N}=\mathcal{M}_{1} \cap \mathcal{N}\right\}$ is a clopen subset of $\mathcal{L} \mathcal{A B} \times \mathcal{L} \mathcal{A B}$.

(d) For any label $\mathcal{M}$ the set $[[\mathcal{M}]]$ of labels contained in $\mathcal{M}$ is a closed subset of $\mathcal{L} \mathcal{A B}$. The set

$$
I N C=\left\{\left(\mathcal{M}_{1}, \mathcal{M}_{2}\right): \mathcal{M}_{1} \subset \mathcal{M}_{2}\right\}
$$

is a closed subset of $\mathcal{L} \mathcal{A B} \times \mathcal{L} \mathcal{A B}$.

(e) The set of finite labels is a countable dense subset of $\mathcal{L} \mathcal{A B}$.

(f) The set of bounded labels is a dense $G_{\delta}$ subset of $\mathcal{L A B}$.

Proof: (a) Every $\mathbf{m} \in \mathcal{B}_{N}$ for some $N$.

(b) If $\mathcal{M} \neq \emptyset$ then $\mathbf{0} \in \mathcal{M} \cap \mathcal{B}_{1}$.

(c) If $\mathcal{N} \subset \mathcal{B}_{N}$ then the $2^{-N}$ ball around $\mathcal{M}$ is either contained in or disjoint from $\left\{\mathcal{M}: \mathcal{M} \cap \mathcal{N}=\mathcal{N}_{1}\right\}$. So $\left\{\mathcal{M}: \mathcal{M} \cap \mathcal{N}=\mathcal{N}_{1}\right\}$ is clopen. With $\mathcal{N}_{1}=0$ or $\mathcal{N}$ these become $\{\mathcal{M}: \mathcal{M} \cap \mathcal{N}=0\}$ and $\{\mathcal{M}: \mathcal{N} \subset \mathcal{M}\}$. Finally, let $\mathcal{N}=\langle\mathbf{m}\rangle$.

The set of pairs such that $\mathcal{M} \cap \mathcal{N}=\mathcal{M}_{1} \cap \mathcal{N}$ is the union of the set of pairs such that $\mathcal{M} \cap \mathcal{N}=\mathcal{N}_{1}=\mathcal{M}_{1} \cap \mathcal{N}$ taken of the finite set of labels $\mathcal{N}_{1} \subset \mathcal{N}$.

(d) The complement of $[[\mathcal{M}]]$ is the union of $\left\{\mathcal{M}_{1}: \mathbf{m} \in \mathcal{M}_{1}\right\}$ as $\mathbf{m}$ varies over $F I N(\mathbb{N}) \backslash \mathcal{M}$. The complement of $I N C$ is the union of $\left\{\mathcal{M}_{1}: \mathbf{m} \in \mathcal{M}_{1}\right\} \times\left\{\mathcal{M}_{2}:\right.$ $\left.\mathbf{m} \notin \mathcal{M}_{2}\right\}$ as $\mathbf{m}$ varies over $F I N(\mathbb{N})$.

(e) $\mathcal{M} \cap \mathcal{B}_{N}$ is a finite label in the $2^{-N}$ ball about $\mathcal{M}$. The set of finite labels is countable since $F I N(\mathbb{N})$ is countable.

(f) For each $\ell,\left\{\mathcal{M}: \rho(\mathcal{M})_{\ell}=\infty\right\}=\bigcap_{k}\{\mathcal{M}: k \chi(\ell) \in \mathcal{M}\}$ is a closed set. So the set of bounded labels is $G_{\delta}$. It is dense because it contains the set of finite labels.

Let $\mathcal{M}^{i}$ be a sequence of labels, or more generally a net of labels with $i$ in a directed set. Define the labels

$$
\begin{aligned}
& \operatorname{LIMSUP}\left\{\mathcal{M}^{i}\right\}=\bigcap_{i} \bigcup_{j \geq i}\left\{\mathcal{M}^{j}\right\}, \\
& \operatorname{LIMINF}\left\{\mathcal{M}^{i}\right\}=\bigcup_{i} \bigcap_{j \geq i}\left\{\mathcal{M}^{j}\right\} .
\end{aligned}
$$

Clearly, $\mathbf{m} \in L I M S U P$ iff frequently $\mathbf{m} \in \mathcal{M}^{i}$ and $\mathbf{m} \in L I M I N F$ iff eventually $\mathbf{m} \in \mathcal{M}^{i}$ and so $L I M I N F \subset L I M S U P$. 
As usual, if we go to a subsequence $\left\{\mathcal{M}^{i^{\prime}}\right\}$ with $L I M S U P^{\prime}$ and $L I M I N F^{\prime}$ then $L I M I N F \subset L I M I N F^{\prime} \subset L I M S U P^{\prime} \subset L I M S U P$.

Proposition 2.8. Let $\left\{\mathcal{M}^{i}\right\}$ be a sequence of labels.

(a) The following are equivalent.

(1) The sequence $\left\{\mathcal{M}^{i}\right\}$ is a convergent sequence.

(2) The sequence $\left\{\mathcal{N}^{i}\right\}$ is a Cauchy sequence.

(3) For every finite label $\mathcal{N}$, the sequence $\left\{\mathcal{M}^{i} \cap \mathcal{N}\right\}$ of finite labels is eventually constant.

(4) For every $\mathbf{m} \in F I N(\mathbb{N})$ either eventually $\mathbf{m} \in \mathcal{M}^{i}$ or eventually $\mathbf{m} \notin \mathcal{M}^{i}$.

(5) $L I M S U P=L I M I N F$.

The common value LIMSUP $=$ LIMINF is then the limit, and is then denoted LIM $\left\{\mathcal{M}^{i}\right\}$.

(b) If $\mathcal{M}^{i} \subset \mathcal{M}^{i+1}$ then $\operatorname{LIM}\left\{\mathcal{M}^{i}\right\}=\bigcup\left\{\mathcal{M}^{i}\right\}$. If $\mathcal{M}^{i} \supset \mathcal{M}^{i+1}$ then $\operatorname{LIM}\left\{\mathcal{M}^{i}\right\}$ $=\bigcap\left\{\mathcal{M}^{i}\right\}$.

Proof: (a) (1) $\Rightarrow(2)$ : Obvious.

$(2) \Rightarrow(3)$ : Since $\mathcal{N} \subset \mathcal{B}_{N}$ for some $N$ this is obvious from the definition of the ultrametric.

$(3) \Rightarrow(4)$ : If $\mathbf{m} \in \mathcal{B}_{N}$ then since $\mathcal{M}^{i} \cap \mathcal{B}_{N}$ is eventually constant, either eventually $\mathbf{m} \in \mathcal{M}^{i}$ or eventually $\mathbf{m} \notin \mathcal{N}^{i}$.

$(4) \Rightarrow(5)$ : (4) says that $\mathbf{m}$ frequently in $\mathcal{M}^{i}$ implies $\mathbf{m}$ is eventually in $\mathcal{M}^{i}$.

$(5) \Rightarrow(1)$ : Assume that $\mathcal{M}=L I M S U P=L I M I N F$. Let $\mathcal{N}_{1}=\mathcal{M} \cap \mathcal{N}$. If $\mathbf{m} \in \mathcal{N}_{1}$ then eventually $\mathbf{m} \in \mathcal{M}^{i}$ and if $\mathbf{m} \in \mathcal{N} \backslash \mathcal{N}_{1}$ then eventually $\mathbf{m} \notin \mathcal{M}^{i}$. Since $\mathcal{N}$ is a finite set it follows that eventually $\mathcal{M}^{i} \cap \mathcal{N}=\mathcal{N}_{1}$.

(b): For an increasing sequence the $L I M S U P=L I M I N F$ is the union and for a decreasing sequence $L I M S U P=L I M I N F$ is the intersection.

The results of part (a) apply more generally to nets. In the metric space $\mathcal{L} \mathcal{A B}$ we need only consider sequences, but later we will be considering pointwise convergence of maps in $\mathcal{L} \mathcal{A} \mathcal{B}^{\mathcal{L A B}}$ and there we will need nets.

Corollary 2.9. The maps defined from $\mathcal{L} \mathcal{A B} \times \mathcal{L} \mathcal{A B}$ to $\mathcal{L} \mathcal{A B}$ by $\left(\mathcal{M}_{1}, \mathcal{M}_{2}\right) \mapsto$ $\mathcal{M}_{1} \cap \mathcal{M}_{2}$ and $\left(\mathcal{M}_{1}, \mathcal{M}_{2}\right) \mapsto \mathcal{M}_{1} \cup \mathcal{M}_{2}$ are continuous.

Proof: Let $\left\{\left(\mathcal{M}_{1}^{i}, \mathcal{M}_{2}^{i}\right)\right\}$ be a sequence converging to $\left(\mathcal{M}_{1}, \mathcal{M}_{2}\right)$ and let $N \in$ $\mathbb{N}$. Eventually, $\mathcal{M}_{1}^{i} \cap \mathcal{B}_{N}=\mathcal{M}_{1} \cap \mathcal{B}_{N}$ and, eventually, $\mathcal{M}_{2}^{i} \cap \mathcal{B}_{N}=\mathcal{M}_{2} \cap \mathcal{B}_{N}$. So, eventually, $\left(\mathcal{M}_{1}^{i} \cup \mathcal{M}_{2}^{i}\right) \cap \mathcal{B}_{N}=\left(\mathcal{M}_{1}^{i} \cap \mathcal{B}_{N}\right) \cup\left(\mathcal{M}_{1}^{i} \cap \mathcal{B}_{N}\right)$ equals $\left(\mathcal{M}_{1} \cup \mathcal{M}_{2}\right) \cap \mathcal{B}_{N}$ and similarly for the intersection. 
Proposition 2.10. Let $\left\{\mathcal{M}^{i}\right\}$ be a sequence of labels.

$\mathbf{m} \in \operatorname{LIMSUP}\left\{\mathcal{M}^{i}\right\}$ iff there is a subsequence $\left\{\mathcal{M}^{i^{\prime}}\right\}$ which is convergent with $\mathbf{m} \in L I M\left\{\mathcal{M}^{i^{\prime}}\right\}$.

$\mathbf{m} \notin L I M I N F\left\{\mathcal{M}^{i}\right\}$ iff there is a subsequence $\left\{\mathcal{M}^{i^{\prime}}\right\}$ which is convergent with $\mathbf{m} \notin L I M\left\{\mathcal{M}^{i^{\prime}}\right\}$.

Proof: The LIMSUP of a subsequence is contained in the LIMSUP of the original sequence and the $L I M I N F$ of a subsequence contains the $L I M I N F$ of the original sequence. Thus, sufficiency is clear in each case.

Let $\left\{\mathbf{m}_{1}, \mathbf{m}_{2}, \ldots\right\}$ be a numbering of the countable set $F I N(\mathbb{N})$ with $\mathbf{m}_{1}=$ $\mathbf{m}$. Since $\mathbf{m} \in \mathcal{M}^{i}$ frequently, we can choose $S E Q_{1}$ an infinite subset of $\mathbb{N}$ so that $\mathbf{m}_{1} \in \mathcal{M}^{i}$ for all $i \in S E Q_{1}$. If eventually $\mathbf{m}_{2} \in \mathcal{M}^{i}$ for $i \in S E Q_{1}$ let these values of $i$ define $S E Q_{2} \subset S E Q_{1}$. Otherwise, let $S E Q_{2}$ be the $i \in S E Q_{1}$ such that $\mathbf{m}_{2} \notin \mathcal{M}^{i}$. Inductively we define a decreasing sequence $S E Q_{n}$ of infinite subsets of $\mathbb{N}$ such that $p \leq n$ implies either $\mathbf{m}_{p} \in \mathcal{M}^{i}$ for all $i \in S E Q_{n}$ or for no $i \in S E Q_{n}$. Diagonalizing, we obtain a convergent subsequence whose limit contains $\mathbf{m}$. That is, if $i_{n}$ be the $n^{\text {th }}$ element of $S E Q_{n}$, then $\left\{M^{i_{n}}\right\}$ is convergent and the limit contains $\mathbf{m}$.

Alternatively, if $\mathbf{m} \notin L I M I N F$ we begin by choosing $S E Q_{1}$ so that $\mathbf{m}_{1} \notin$ $\mathcal{M}^{i}$ for all $i \in S E Q_{1}$ and continue as before.

Corollary 2.11. $\mathcal{L} \mathcal{A B}$ is a compact, zero-dimensional metric space with $\emptyset$ the only isolated point.

Proof: By Propositions 2.10 every sequence in $\mathcal{L} \mathcal{A B}$ has a convergent subsequence, i.e. the metric space satisfies the Bolzano-Weierstrass property and so is compact. It is zero-dimensional because it has an ultrametric. If $\mathcal{N}$ is a finite, nonempty label then $\mathcal{N} \cup\{\chi(\ell)\}$ is a sequence of finite labels which are eventually distinct. The sequence converges to $\mathcal{N}$ as $\ell \rightarrow \infty$ and so $\mathcal{N}$ is not an isolated point. Since the set of finite labels is dense, it follows that no nonempty label is isolated.

Lemma 2.12. Let $\Phi$ be a compact subset of $\mathcal{L} \mathcal{A B}$. If $\left\{\mathbf{m}^{i}: i \in \mathbb{N}\right\}$ is a nondecreasing sequence in $\bigcup \Phi$ then there exists $\mathcal{M} \in \Phi$ such that $\mathbf{m}^{i} \in \mathcal{M}$ for all $i$.

Proof: Assume $\mathbf{m}^{i} \in \mathcal{M}^{i} \in \Phi$ for all $i$. By compactness some subsequence $\left\{\mathcal{M}^{i^{\prime}}\right\}$ converges to $\mathcal{M} \in \Phi$. For each $k, i^{\prime}>k$ implies $\mathbf{m}^{k} \leq \mathbf{m}^{i^{\prime}} \in \mathcal{M}^{i^{\prime}}$. That is, each $\mathbf{m}^{k}$ is eventually in $\mathcal{M}^{i^{\prime}}$ as $i^{\prime} \rightarrow \infty$. Hence, $\mathbf{m}^{k} \in \mathcal{M}$ for all $k$. 
Proposition 2.13. Let $\mathcal{L} \subset \mathcal{L} \mathcal{A B}$ be either the set of bounded labels or the set of labels of finite type. A subset $\Phi \subset \mathcal{L}$ is compact iff $\Phi$ is closed in the relative topology of $\mathcal{L}$ and $\bigcup \Phi \in \mathcal{L}$. In particular, the union of a compact set of bounded labels is a bounded label.

Proof: If $\bigcup \Phi \in \mathcal{L}$ then the compact set $[[\bigcup \Phi]]$ is contained in $\mathcal{L}$ and since $\Phi \subset[[\bigcup \Phi]]$ is closed relative to $\mathcal{L}$, it is closed relative to [[U $\Phi]]$ and so is itself compact.

Now assume that $\Phi$ is compact.

If $\bigcup \Phi$ is not bounded then for some $\ell \in \mathbb{N}$ the strictly increasing sequence $\left\{\mathbf{m}^{i}=i \chi(\ell)\right\}$ is in $\bigcup \Phi$ and so Lemma 2.12 implies that the sequence is contained in some $\mathcal{M} \in \Phi$ and so $\mathcal{M}$ is unbounded.

Similarly, if $\bigcup \Phi$ is not of finite type then there exists a strictly increasing sequence $\left\{\mathbf{m}^{i}\right\}$ in $\bigcup \Phi$ and so again Lemma 2.12 implies that the sequence is contained in some $\mathcal{M} \in \Phi$ and so $\mathcal{M}$ is not of finite type.

In each case the contrapositive says that $\Phi \subset \mathcal{L}$ implies $\bigcup \Phi \in \mathcal{L}$.

\subsection{The action of $F I N(\mathbb{N})$ on $\mathcal{L} \mathcal{A B}$.}

Given an $\mathbb{N}$-vector $\mathbf{r}$ we define the map $P_{\mathbf{r}}$ on $\mathcal{L} \mathcal{A B}$, by $P_{\mathbf{r}}(\mathcal{M})=\mathcal{M}-\mathbf{r}$.

Proposition 2.14. For each $\mathbf{r} \in F I N(\mathbb{N})$ the function $P_{\mathbf{r}}$ on $\mathcal{L} \mathcal{A B}$ is continuous. In particular, if $\left\{\mathcal{M}^{i}\right\}$ is a convergent sequence of labels then $\left\{\mathcal{M}^{i}-\mathbf{r}\right\}$ is convergent with $\operatorname{LIM}\left\{\mathcal{M}^{i}-\mathbf{r}\right\}=\operatorname{LIM}\left\{\mathcal{M}^{i}\right\}-\mathbf{r}$.

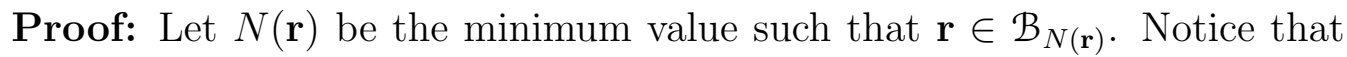
$N(\mathbf{m}+\mathbf{r}) \leq N(\mathbf{m})+N(\mathbf{r})$ because $\mathbf{m}+\mathbf{r}<N(\mathbf{m})+N(\mathbf{r})$ and supp $\mathbf{m}+\mathbf{r}=$ $($ supp $\mathbf{m}) \cup(\operatorname{supp} \mathbf{r}) \subset[1, \max (N(\mathbf{m}), N(\mathbf{r}))]$.

It follows that for labels $\mathcal{M}_{1}, \mathcal{M}_{2}$

$$
\begin{gathered}
\mathcal{M}_{1} \cap \mathcal{B}_{N+N(\mathbf{r})}=\mathcal{M}_{2} \cap \mathcal{B}_{N+N(\mathbf{r})} \\
\Longrightarrow \quad\left(\mathcal{M}_{1}-\mathbf{r}\right) \cap \mathcal{B}_{N}=\left(\mathcal{M}_{2}-\mathbf{r}\right) \cap \mathcal{B}_{N} .
\end{gathered}
$$

For if $\mathbf{m} \in\left(\mathcal{M}_{1}-\mathbf{r}\right) \cap \mathcal{B}_{N}$ then $\mathbf{m}+\mathbf{r} \in \mathcal{M}_{1} \cap \mathcal{B}_{N+N(\mathbf{r})}=\mathcal{M}_{2} \cap \mathcal{B}_{N+N(\mathbf{r})}$. Hence, $\mathbf{m} \in \mathcal{M}_{2}-\mathbf{r}$. Since $\mathbf{m} \in \mathcal{B}_{N}$, it follows that $\mathbf{m} \in\left(\mathcal{M}_{2}-\mathbf{r}\right) \cap \mathcal{B}_{N}$. Symmetrically for reverse inclusion.

From (2.8) it follows that $d\left(\mathcal{M}_{1}, \mathcal{M}_{2}\right)<2^{-N-N(\mathbf{r})}$ implies $d\left(P_{\mathbf{r}}\left(\mathcal{M}_{1}\right), P_{\mathbf{r}}\left(\mathcal{M}_{2}\right)\right)$ $<2^{-N}$. This shows that $P_{\mathbf{r}}$ is Lipschitz with Lipschitz constant at most $2^{N(\mathbf{r})}$. 
Corollary 2.15. The map $P: F I N(\mathbb{N}) \times \mathcal{L A} \mathcal{A B} \rightarrow \mathcal{L} \mathcal{A B}$ given by $(\mathbf{r}, \mathcal{M}) \mapsto$ $\mathcal{M}-\mathbf{r}=P_{\mathbf{r}}(\mathcal{M})$ is a continuous monoid action of $F I N(\mathbb{N})$ on $\mathcal{L} \mathcal{A B}$.

The action is faithful i.e. if $P_{\mathbf{r}}(\mathcal{M})=P_{\mathbf{s}}(\mathcal{M})$ for all $\mathcal{M}$ then $\mathbf{r}=\mathbf{s}$.

Proof: It is an action since $\left(\mathcal{M}-\mathbf{r}_{1}\right)-\mathbf{r}_{2}=\mathcal{M}-\left(\mathbf{r}_{1}+\mathbf{r}_{2}\right)$ for $\mathbb{N}$-vectors $\mathbf{r}_{1}, \mathbf{r}_{2}$ and $\mathcal{M}-\mathbf{0}=\mathcal{M}$. It is a continuous action by Proposition 2.14.

For a label $\mathbf{r}$ let $\mathcal{M}$ be the finite label $\langle\mathbf{r}\rangle$. Since $\{\mathbf{r}\}=\max \mathcal{M}, P_{\mathbf{r}}(\mathcal{M})=0$. If $P_{\mathbf{s}}(\mathcal{M})=0$ then $\mathbf{s} \in \mathcal{M}$ and so $\mathbf{s} \leq \mathbf{r}$. Clearly, $\mathbf{r}-\mathbf{s} \in P_{\mathbf{s}}(\mathcal{M})$ and so $\mathbf{r}-\mathbf{s}=\mathbf{0}$.

Notice that $F I N(\mathbb{N})$ is the free abelian monoid generated by $\{\chi(\ell): \ell \in \mathbb{N}\}$ and it is a submonoid of the free abelian group consisting of the members of $\mathbb{Z}^{\mathbb{N}}$ with finite support. Furthermore, $\mathbf{r}_{1}+\mathbf{s}=\mathbf{r}_{2}+\mathbf{s}$ implies $\mathbf{r}_{1}=\mathbf{r}_{2}$. In particular, $\mathbf{r}+\mathbf{r}=\mathbf{r}$ only when $\mathbf{r}=\mathbf{0}$. Also, $\mathbf{r}+\mathbf{s}=\mathbf{0}$ iff $\mathbf{r}=\mathbf{s}=\mathbf{0}$. Thus, $F I N(\mathbb{N})$ is a cancelation monoid without inverses and Lemmas C.7 and C.8 apply to $F I N(\mathbb{N})$.

Giving $F I N(\mathbb{N})$ the discrete topology, we obtain on the Stone-Čech compactification $\beta F I N(\mathbb{N})$ the structure of an Ellis semigroup with product which extends the addition on $F I N(\mathbb{N})$ and is such that $Q \mapsto Q R$ is continuous for any $R \in \beta F I N(\mathbb{N})$. Let $\beta^{\prime} F I N(\mathbb{N})=\beta F I N(\mathbb{N}) \backslash\{0\}$ and $\beta^{*} F I N(\mathbb{N})=$ $\beta F I N(\mathbb{N}) \backslash F I N(\mathbb{N})$. Notice that since $F I N(\mathbb{N})$ is discrete, it is the set of isolated points in $\beta F I N(\mathbb{N})$. The elements of $F I N(\mathbb{N})$ are continuous on $\beta F I N(\mathbb{N})$ and commute with all elements of $\beta F I N(\mathbb{N})$. Thus, the submonoid $F I N(\mathbb{N})$ acts continuously on $\beta F I N(\mathbb{N})$. The action of $F I N(\mathbb{N})$ extends to an Ellis action of $\beta F I N(\mathbb{N})$ on $\mathcal{L} \mathcal{A B}$. See Appendix $\mathrm{C}$

Proposition 2.16. ((a) If $\mathcal{N} \subset \mathcal{M}$ then $Q(\mathcal{N}) \subset Q(\mathcal{M}) \subset \mathcal{M}$ for all $Q \in$ $\beta F I N(\mathbb{N})$.

(b) The sets $\beta^{\prime} F I N(\mathbb{N})$ and $\beta^{*} F I N(\mathbb{N})$ are closed, $F I N(\mathbb{N})$ invariant subsets of $\beta F I N(\mathbb{N})$ and so are ideals in the Ellis semigroup.

(c) Every nonempty, closed sub-semigroup of $\beta F I N(\mathbb{N})$ contains an idempotent and all the idempotents of $\beta^{\prime} F I N(\mathbb{N})$ lie in $\beta^{*} F I N(\mathbb{N})$.

(d) For all $Q \in \beta F I N(\mathbb{N}), Q(\mathcal{M})=F I N(\mathbb{N})$ iff $\mathcal{M}=F I N(\mathbb{N})$.

Proof: (a) Clearly, $\mathcal{N} \subset \mathcal{M}$ implies $P_{r}(\mathcal{N}) \subset P_{r}(\mathcal{M}) \subset \mathcal{M}$ and the inclusions are preserved in the limit by Lemma 2.7.

(b) See Lemmas C.7 and C.8 .

(c) The existence of idempotents is the Ellis-Numakura Lemma, Lemma B.1. We saw above that $\mathbf{0}$ is the only idempotent in $F I N(\mathbb{N})$ and so there are no idempotents in $\beta^{\prime} F I N(\mathbb{N}) \backslash \beta^{*} F I N(\mathbb{N})$.

$(\mathrm{d})$ For all $\mathbf{r}, P_{\mathbf{r}}(F I N(\mathbb{N}))=F I N(\mathbb{N})$ and so $F I N(\mathbb{N})$ is fixed by all $Q \in$ $\beta F I N(\mathbb{N})$. If $\mathcal{M} \neq F I N(\mathbb{N})$ then $Q(\mathcal{M}) \subset \mathcal{M}$ and so does not equal $F I N(\mathbb{N})$. 
Recall that

$$
\begin{aligned}
& \mathbf{r} \in \mathcal{M} \quad \Longleftrightarrow \quad \mathbf{0} \in P_{\mathbf{r}}(\mathcal{M}) . \\
& \mathbf{r} \notin \mathcal{M} \quad \Longleftrightarrow \quad \emptyset=P_{\mathbf{r}}(\mathcal{M}) .
\end{aligned}
$$

For a discrete set $\Gamma$, a subset $A$ of $\Gamma$ and its complement have disjoint closures in $\beta \Gamma$ because the characteristic function of $A$ extends to a continuous function on $\beta \Gamma$. It follows that for any label $\mathcal{M}$, the closure in $\beta F I N(\mathcal{M})$ of $\mathcal{M} \subset$ $F I N(\mathcal{M})$ is the clopen set $\{Q \in \beta F I N(\mathbb{N}): 0 \in Q(\mathcal{M})\}$ with complement $\{Q \in \beta F I N(\mathbb{N}): \emptyset=Q(\mathcal{M})\}$. In particular, if $\mathcal{M}$ is a finite label then $Q(\mathcal{M})=\emptyset$ for all $Q \in \beta^{*} F I N(\mathbb{N})$.

Let $\Theta(\mathcal{M})$ be the closure in the space of labels of the set $\left\{P_{\mathbf{r}}(\mathcal{M})=\mathcal{M}-\mathbf{r}: \mathbf{r}\right.$ an $\mathbb{N}$-vector $\}$. That is, $\Theta(\mathcal{M})$ is the orbit closure of $\mathcal{M}$ with respect to the $F I N(\mathbb{N})$ action or, equivalently, $\Theta(\mathcal{M})=\beta F I N(\mathbb{N}) \cdot \mathcal{M}$. Since $[[\mathcal{M}]]$ is closed and invariant, $\Theta(\mathcal{M}) \subset[[\mathcal{M}]]$. Even in the finite case, it can happen that the inclusion is proper.

Example 2.17. Set $\mathcal{M}=\langle\chi(1)+\chi(2), 2 \chi(2)+\chi(3)\rangle$, and let $\mathcal{N}=\langle\chi(1)+$ $\chi(2), \chi(2)+\chi(3)\rangle$. It is easy to check that $\mathcal{N} \in[[\mathcal{M}]] \backslash \Theta(\mathcal{M})$.

For $F I N(\mathbb{N}), P_{\mathbf{r}}(F I N(\mathbb{N}))=F I N(\mathbb{N})$ for all $\mathbf{r} \in F I N(\mathbb{N})$ implies $\Theta(F I N(\mathbb{N}))$ $=\{F I N(\mathbb{N})\}$.

The action of $F I N(\mathbb{N})$ on $\mathcal{L} \mathcal{A B}$ restricts to an action on the closed invariant set $\Theta(\mathcal{M})$ for any label $\mathcal{M}$.

Lemma 2.18. (a) For any label $\mathcal{M} \neq F I N(\mathbb{N}), \emptyset \in \Theta(\mathcal{M})$.

(b)If $\mathcal{M}$ is nonempty and bounded then $0 \in \Theta(\mathcal{M})$.

(c) If $\mathcal{M}$ is nonempty and bounded then the action of $F I N(\mathbb{N})$ on $\Theta(\mathcal{M})$ is weakly faithful. In fact, $P_{\mathbf{r}}(\mathcal{M})=\mathcal{M}$ only for $\mathbf{r}=0$.

Proof: (a) : If $\mathbf{r} \notin \mathcal{M}$ then $\mathcal{M}-\mathbf{r}=\emptyset$ and so $\emptyset \in \Theta(\mathcal{M})$.

(b): If $\mathbf{r} \in \max \mathcal{M}$ then $\mathcal{M}-\mathbf{r}=0$.

Now assume that $\mathcal{M}$ is bounded so that each $\mathcal{M} \wedge[1, i]$ is a finite label. Let $\mathbf{r}^{i}$ be maximal element of $\mathcal{M} \wedge[1, i]$. Clearly $\mathbf{0} \in \operatorname{LIMINF}\left\{\mathcal{M}-\mathbf{r}^{i}\right\}$. On the other hand if $\mathbf{w} \in L I M S U P\left\{\mathcal{M}-\mathbf{r}^{i}\right\}$ then for some $j$ we have supp $\mathbf{w} \subset[1, j]$. Frequently $\mathbf{w}+\mathbf{r}^{i} \in \mathcal{M}$, and so there exists $i \geq j, \mathbf{w}+\mathbf{r}^{i} \in \mathcal{M} \wedge[1, i]$. Maximality implies $\mathbf{w}=\mathbf{0}$. That is, $0=\operatorname{LIM}\left\{\mathcal{M}-\mathbf{r}^{i}\right\}$.

(c): Proposition 2.5(d) implies that $P_{\mathbf{r}}(\mathcal{M})$ is a proper subset of $\mathcal{M}$ when $\mathcal{M}$ is bounded and non-empty and $\mathbf{r}>0$.

Remark: Notice that $P_{\mathbf{r}}(\mathcal{M})=0$ iff $\mathbf{r} \in \max \mathcal{M}$ and so if $\max \mathcal{M}=\emptyset$ then $\mathcal{M}-\mathbf{r} \neq 0$ for any $\mathbf{r} \in F I N(\mathbb{N})$. 
Let $\Theta^{\prime}(\mathcal{M})$ be the closure in the space of labels of the set $\{\mathcal{M}-\mathbf{r}: \mathbf{r} \in$ $F I N(\mathbb{N})$ with $\mathbf{r}>\mathbf{0}\}$. Thus, $\Theta(\mathcal{M})=\Theta^{\prime}(\mathcal{M}) \cup\{\mathcal{M}\}$ and $\Theta^{\prime}(\mathcal{M})=\beta^{\prime} F I N(\mathbb{N})$. $\mathcal{M}$.

Definition 2.19. Call $\mathcal{M}$ a recurrent label if $\mathcal{M} \in \Theta^{\prime}(\mathcal{M})$. Equivalently, $\mathcal{M}$ is recurrent if there exists a sequence $\left\{\mathbf{r}^{i}>\mathbf{0}\right\}$ such that $\mathcal{M}=\operatorname{LIM}\left\{\mathcal{M}-\mathbf{r}^{i}\right\}$ and so for all $\mathbf{m} \in \mathcal{M}$ eventually $\mathbf{m}+\mathbf{r}^{i} \in \mathcal{M}$.

This is the notion of recurrence for the system $(\mathcal{L A B}, F I N(\mathbb{N}))$ as defined in Section 1.

If $\mathbf{m} \in \max \mathcal{M}$ then $\{\mathcal{N}: \mathbf{m} \in \mathcal{N}\}$ is a clopen subset of $\mathcal{L} \mathcal{A B}$ which contains $\mathcal{M}$ and is disjoint from $\Theta^{\prime}(\mathcal{M})$. So if $\mathcal{M}$ is a recurrent label then $\max \mathcal{M}=\emptyset$. In particular, if $\mathcal{M}$ is of finite type and nonempty then $\mathcal{M} \notin \Theta^{\prime}(\mathcal{M})$ and so is not recurrent.

For example, $\mathcal{M}=F I N(\mathbb{N})$ is a recurrent label since then $\mathcal{M}=P_{\mathbf{r}}(\mathcal{M})$ for all $\mathbf{r} \in \mathcal{M}$. By Proposition 2.5 (d) $P_{\mathbf{r}}(\mathcal{M})$ is a proper subset of $\mathcal{M}$ when $\mathcal{M}$ is bounded and $\mathbf{r}>\mathbf{0}$ and so then cannot equal $\mathcal{M}$. Nonetheless, there are bounded recurrent labels.

For example, if $\mathcal{M}=\langle\chi(L)\rangle$ for some infinite $L \subset \mathbb{N}$ then

$$
\mathcal{M}=\operatorname{LIM}\left\{\mathcal{M}-\chi\left(\ell^{i}\right)\right\}
$$

when $\left\{\ell^{i}\right\}$ is a sequence of distinct elements of $L$.

Define the isotropy set of $\mathcal{M}$

$$
I S O(\mathcal{M})=\{Q \in \beta F I N(\mathbb{N}): Q(\mathcal{M})=\mathcal{M}\}
$$

Proposition 2.20. Let $\mathcal{M}$ be a label.

(a) $I S O(\mathcal{M})$ is a closed submonoid of $\beta F I N(\mathbb{N})$ and for $Q_{1}, Q_{2} \in \beta F I N(\mathbb{N})$ the product $Q_{1} Q_{2}$ is in $I S O(\mathcal{M})$ iff both $Q_{1}$ and $Q_{2}$ are in $I S O(\mathcal{M})$.

(b) The following are equivalent.

(i) $\mathcal{M}$ is recurrent.

(ii) There exists $Q \in \beta^{\prime} F I N(\mathbb{N})$ such that $Q(\mathcal{M})=\mathcal{M}$.

(iii) $I S O(\mathcal{M}) \cap \beta^{\prime} F I N(\mathbb{N})$ is a nonempty, closed subsemigroup of $\beta F I N(\mathbb{N})$.

(iv) There exists an idempotent $Q \in \beta^{*} F I N(\mathbb{N})$ such that $Q(\mathcal{M})=\mathcal{M}$.

(c) If $Q$ is an idempotent in $\beta^{*} F I N(\mathbb{N})$ then $Q(\mathcal{M})$ is a recurrent element of $\Theta^{\prime}(\mathcal{M})$. In particular, if $\mathcal{M}$ is of finite type then $Q(\mathcal{M})=\emptyset$.

(d) If $\mathcal{N}$ is a recurrent label with $\mathcal{N} \subset \mathcal{M}$, then there is a recurrent label $\mathcal{M}_{1} \in \Theta(\mathcal{M})$ such that $\mathcal{N} \subset \mathcal{M}_{1}$.

(e) If $\mathbf{r} \in F I N(\mathbb{N})$, then $I S O(\mathcal{M}) \subset I S O\left(P_{\mathbf{r}}(\mathcal{M})\right)$. So if $\mathcal{M}$ is a recurrent label then $P_{\mathbf{r}}(\mathcal{M})$ is a recurrent label.

Proof: (a) Since $Q \mapsto Q(\mathcal{M})$ is continuous, $I S O(\mathcal{M})$ is closed. It is clearly closed under composition and so is a semigroup. Since $P_{\mathbf{0}}=i d, I S O(\mathcal{M})$ contains $\mathbf{0}$ and so is a monoid. 
For $Q, P \in \beta F I N(\mathbb{N})$ if $Q(\mathcal{M}) \neq \mathcal{M}$ then $\mathcal{N}=Q(\mathcal{M})$ is a proper subset of $\mathcal{M}$. It follows that $P Q(\mathcal{N})=P(\mathcal{N}) \subset \mathcal{N}$ and so it,too, is a proper subset of $\mathcal{M}$ for any $P$. Also, $P(\mathcal{M}) \subset \mathcal{M}$ and so $Q P(\mathcal{M}) \subset Q(\mathcal{M})=\mathcal{N}$ is also a proper subset of $\mathcal{M}$. Thus, $\{Q \in \beta F I N(\mathbb{N}): Q(\mathcal{M}) \neq \mathcal{M}\}$ is a two-sided ideal (though it is not closed when $\mathcal{M}$ is recurrent). It follows that $Q_{1} Q_{2}(\mathcal{M})=\mathcal{M}$ implies $Q_{1}(\mathcal{M})=\mathcal{M}$ and $Q_{2}(\mathcal{M})=\mathcal{M}$.

(b) (i) $\Rightarrow$ (ii): If $\left\{\mathcal{M}-\mathbf{r}^{i}\right\} \rightarrow \mathcal{M}$ with all $\mathbf{r}^{i}>0$ then $Q(\mathcal{M})=\mathcal{M}$ for any limit point in $\beta F I N(\mathbb{N})$ of the sequence $\left\{P_{\mathbf{r}^{i}}\right\}$. Since, 0 is an isolated point of $\beta F I N(\mathbb{N})$, it follows that $Q \in \beta^{\prime} F I N(\mathbb{N})$.

(ii) $\Rightarrow$ (iii): As the intersection of two closed subsemigroups, $I S O(\mathcal{M}) \cap$ $\beta^{\prime} F I N(\mathbb{N})$ is a closed subsemigroup. It is nonempty by (ii).

(iii) $\Rightarrow$ (iv): The nonempty, closed subsemigroup $I S O(\mathcal{M}) \cap \beta^{\prime} F I N(\mathbb{N})$ contains an idempotent which cannot lie in $F I N(\mathbb{N}) \backslash\{\mathbf{0}\}$ and so is in $\beta^{*} F I N(\mathbb{N})$.

(iv) $\Rightarrow(\mathrm{i}): \Theta^{\prime}(\mathcal{M})=\beta^{\prime} F I N(\mathbb{N}) \cdot \mathcal{M}$ and so $\mathcal{M} \in \Theta^{\prime}(\mathcal{M})$ if a nonzero idempotent fixes $\mathcal{M}$.

(c): If $Q \in \beta^{\prime} F I N(\mathbb{N})$ then $Q(\mathcal{M}) \in \Theta^{\prime}(\mathcal{M})$. If $Q$ is an idempotent then $Q(Q(\mathcal{M}))=Q(\mathcal{M})$ implies that $Q(\mathcal{M})$ is recurrent. If $\mathcal{M}$ is of finite type, then every nonempty label in $[[\mathcal{M}]]$ is of finite type and so is not recurrent. It follows that when $\mathcal{M}$ is of finite type $Q(\mathcal{M})$ must be empty.

(d): If $\mathcal{N}$ is recurrent then there exists an idempotent $Q \in \beta^{*} F I N(\mathbb{N})$ such that $Q(\mathcal{N})=\mathcal{N}$. Since $\mathcal{N} \subset \mathcal{M}, \mathcal{N}=Q(\mathcal{N}) \subset Q(\mathcal{M}) \subset \mathcal{M}$. Let $\mathcal{M}_{1}=Q(\mathcal{M})$.

(e): If $Q(\mathcal{M})=\mathcal{M}$ then $Q\left(P_{\mathbf{r}}(\mathcal{M})\right)=P_{\mathbf{r}}(Q(\mathcal{M}))=P_{\mathbf{r}}(\mathcal{M})$ because $Q$ and $P_{\mathbf{r}}$ commute.

Definition 2.21. Call $\mathcal{M}$ a strongly recurrent label if $\mathcal{M}$ is bounded and infinite and for every $\mathbf{m} \in \mathcal{M}$, there is a finite set $F(\mathbf{m}) \subset \mathbb{N}$ such that $\mathcal{M}-\mathbf{m} \supset$ $\{\mathbf{w} \in \mathcal{M}:(\operatorname{supp} \mathbf{w}) \cap F(\mathbf{m})=\emptyset\}$.

Call a label $\mathcal{N}$ a strongly recurrent set for a bounded label $\mathcal{M}$ if $\mathcal{N}$ is infinite, $\mathcal{N} \subset \mathcal{M}$ and for every $\mathbf{m} \in \mathcal{M}$, there is a finite set $F(\mathbf{m}) \subset \mathbb{N}$ such that $\mathcal{M}-\mathbf{m} \supset\{\mathbf{w} \in \mathcal{N}:(\operatorname{supp} \mathbf{w}) \cap F(\mathbf{m})=\emptyset\}$. Thus, $\mathcal{M}$ is strongly recurrent iff $\mathcal{M}$ itself is a strongly recurrent set for $\mathcal{M}$.

Notice that $\mathcal{N} \subset \mathcal{M}$ implies that $\mathcal{N}$ is bounded and $\mathcal{M}$ is infinite.

Proposition 2.22. Let $\mathcal{M}$ be a nonempty label.

(a) If $\mathcal{M}=\operatorname{LIM}\left\{\mathcal{M}-\mathbf{r}^{i}\right\}$ and $\mathcal{M}$ is bounded, then LIMSUP supp $\left.\mathbf{r}^{i}\right\}=$ $\emptyset$. If, in addition, $\mathbf{r}^{i}>\mathbf{0}$ for all $i$ then $\bigcup_{i}$ supp $\mathbf{r}^{i}$ is infinite.

(b) Assume $\mathcal{N}$ is a strongly recurrent set for a bounded label $\mathcal{M}$. If $\left\{\mathbf{r}^{i}\right\}$ is a sequence of elements of $\mathcal{N}$ such that LIMSUP $\left\{\right.$ supp $\left.\mathbf{r}^{i}\right\}=\emptyset$, then 
$\mathcal{M}=L I M\left\{\mathcal{M}-\mathbf{r}^{i}\right\}$. In particular, $\mathcal{M}$ is recurrent if it has a strongly recurrent set, and every strongly recurrent label is recurrent.

(c) Assume $\mathcal{M}$ is bounded and infinite. $\mathcal{M}$ is strongly recurrent iff for every sequence $\left\{\mathbf{r}^{i}\right\}$ of elements of $\mathcal{M}, L I M S U P\left\{\right.$ supp $\left.\mathbf{r}^{i}\right\}=\emptyset$ implies $\mathcal{M}=\operatorname{LIM}\left\{\mathcal{M}-\mathbf{r}^{i}\right\}$.

(d) If $\mathcal{M}$ is bounded and recurrent then there exists an infinite set $L \subset \mathbb{N}$ such that $\langle\chi(L)\rangle$ is a strongly recurrent set for $\mathcal{M}$.

(e) The following conditions are equivalent.

(i) If $\mathbf{m}_{1}, \mathbf{m}_{2} \in \mathcal{M}$ with disjoint supports then $\mathbf{m}_{1}+\mathbf{m}_{2} \in \mathcal{M}$.

(ii) $\mathcal{M}$ is a sublattice of FIN $(\mathbb{N})$, i.e. if $\mathbf{m}_{1}, \mathbf{m}_{2} \in \mathcal{M}$ then $\mathbf{m}_{1} \vee \mathbf{m}_{2} \in$ $\mathcal{M}$.

(iii) $\mathcal{M}=\langle\rho\rangle$ for some $\rho \in \mathbb{Z}_{+\infty}^{\mathbb{N}}$.

(iv) $\mathcal{M}=\langle\rho(\mathcal{M})\rangle$.

If these conditions hold, and, in addition, $\mathcal{M}$ is bounded and infinite, then $\mathcal{M}$ is strongly recurrent.

Proof: (a) If $\ell \in \operatorname{supp} \mathbf{r}^{i}$, then $\rho(\mathcal{M})_{\ell} \cdot \chi(\ell) \in \mathcal{M} \backslash\left(\mathcal{M}-\mathbf{r}^{i}\right)$. So it cannot happen that $\ell \in \operatorname{supp} \mathbf{r}^{i}$ infinitely often. Thus, for any $\ell \in \mathbb{N}$, eventually $\ell \notin \operatorname{supp} \mathbf{r}^{i}$.

If $\mathbf{r}^{i}>\mathbf{0}$ then supp $\mathbf{r}^{i}$ is nonempty. If an infinite sequence of nonempty subsets of $\mathbb{N}$ has a finite union, then some $\ell \in \mathbb{N}$ must lie in infinitely many of them.

(b) For any $\mathbf{m} \in \mathcal{M}, \mathbf{m} \in \mathcal{M}-\mathbf{r}^{i}$ as soon as $F(\mathbf{m}) \cap$ supp $\mathbf{r}^{i}=\emptyset$ which happens eventually since $L I M S U P\left\{\operatorname{supp} \mathbf{r}^{i}\right\}=\emptyset$.

(c) If $\mathcal{M}$ is bounded and infinite but not strongly recurrent then there exists $\mathbf{m} \in \mathcal{M}$ and for every $F$ finite subset of $\mathbb{N}$ there exists $\mathbf{n} \in \mathcal{M}$ with supp $\mathbf{n} \cap$ $F=\emptyset$ but with $\mathbf{m}+\mathbf{n} \notin \mathcal{M}$. Note that this implies $\mathbf{n}>\mathbf{0}$.

Let $F_{1}=\operatorname{supp} \mathbf{m}$ and choose a positive $\mathbf{r}_{1} \in \mathcal{M}$ with support disjoint from $F_{1}$ and is such that $\mathbf{m}+\mathbf{r}^{1} \notin \mathcal{M}$. Let $F_{2}=F_{1} \cup \operatorname{supp} \mathbf{r}^{1}$. Inductively, we build an increasing sequence of finite sets $\left\{F^{i}\right\}$ and positive elements $\mathbf{r}^{i} \in \mathcal{M}$ such that supp $\mathbf{r}^{i} \subset F^{i+1} \backslash F^{i}$ and $\mathbf{m}+\mathbf{r}^{i} \notin \mathcal{M}$. Since the supports are disjoint, $L I M S U P\left\{\right.$ supp $\left.\mathbf{r}^{i}\right\}=\emptyset$. Because $\mathbf{m} \notin L I M\left\{\mathcal{M}-\mathbf{r}^{i}\right\}$, the limit is not $\mathcal{M}$.

The converse follows from (b).

(d) Since $\mathcal{M}$ is recurrent there exists a sequence $\left\{\mathbf{r}^{i}>\mathbf{0}\right\}$ be such that $\mathcal{M}=L I M\left\{\mathcal{M}-\mathbf{r}^{i}\right\}$. Since $\mathcal{M}$ is bounded, it follows from (a) that $\bigcup_{i} \operatorname{supp} \mathbf{r}^{i}$ is infinite.

Choose $\ell_{1} \in \operatorname{supp} \mathbf{r}^{1}$ and let $i_{1}=1$ and $\mathcal{N}_{1}=\left\{\chi\left(\ell_{1}\right)\right\} \cup \mathcal{M} \wedge[1,1]$.

There exists $\mathbf{r}^{i_{2}}$ with $\mathbf{r}_{\ell_{2}}^{i_{2}}>0$ and $\ell_{2}$ not in the support of a member of $\mathcal{N}_{1}$ and is such that $\mathbf{m}+\mathbf{r}^{i_{2}} \in \mathcal{M}$ for all $\mathbf{m} \in \mathcal{N}_{1}$. Let $\mathcal{N}_{2}=\left\{\mathbf{m}+\chi\left(\ell_{2}\right): \mathbf{m} \in\right.$ $\left.\mathcal{N}_{1}\right\} \cup \mathcal{M} \wedge[1,2]$. 
Inductively, we can choose $\ell_{k+1}$ such that $\mathbf{m}+\chi\left(\ell_{k+1}\right) \in \mathcal{M}$ for all $\mathbf{m} \in \mathcal{N}_{k}$ and with $\ell_{k+1}$ not in the support of any member of $\mathcal{N}_{k}$. Let $\mathcal{N}_{k+1}=\{\mathbf{m}+$ $\left.\chi\left(\ell_{k+1}\right): \mathbf{m} \in \mathcal{N}_{k}\right\} \cup \mathcal{M} \wedge[1, k+1]$.

By the inductive construction if $\mathbf{m} \in \mathcal{N}_{k-1}$ then $\mathbf{m}+\Sigma_{i=k}^{j} \chi\left(\ell_{i}\right) \in \mathcal{M}$ for all $j \geq k$. With $\mathbf{m}=0$ this says that $\Sigma_{i=1}^{j} \chi\left(\ell_{i}\right) \in \mathcal{M}$ for all $j$.

Let $L=\left\{\ell_{k}\right\}$ and $\mathcal{N}=\langle\chi(L)\rangle$. For any $\mathbf{m} \in \mathcal{M}$ there exists $k$ such that $\mathbf{m} \in \mathcal{N}_{k-1}$. Let $F(\mathbf{m})=\left[1, \ell_{k-1}\right]$. If $\mathbf{w} \in \mathcal{N}$ with support disjoint from $F(\mathbf{m})$ then $\mathbf{w} \leq \Sigma_{i=k}^{j} \chi\left(\ell_{i}\right)$ for some $j>k$. Hence, $\mathbf{w}+\mathbf{m} \in \mathcal{M}$ and so $\mathcal{N}$ is a strongly recurrent set for $\mathcal{M}$.

(e) (iv) $\Rightarrow$ (iii) $\Rightarrow$ (ii): Obvious.

(ii) $\Rightarrow$ (i): If $\mathbf{m}_{1}$ and $\mathbf{m}_{2}$ have disjoint supports then $\mathbf{m}_{1} \vee \mathbf{m}_{2}=\mathbf{m}_{1}+\mathbf{m}_{2}$.

(i) $\Rightarrow$ (iv): For any $\mathcal{M}$ and $n \in \mathbb{N}, n \leq \rho(\mathcal{M})_{\ell}$ implies $n \chi(\ell) \in \mathcal{M}$. So if $\mathbf{m} \leq$ $\rho(\mathcal{M})$ then (i) (and induction) implies that $\mathbf{m}=\Sigma\left\{\mathbf{m}_{\ell} \chi(\ell): \ell \in \operatorname{supp} \mathbf{m}\right\}$ is in $\mathcal{M}$.

Now assume that $\mathcal{M}$ is bounded and infinite so that $\bigcup$ Supp $\mathcal{M}$. For any $\mathbf{m} \in \mathcal{M}$, let $F(\mathbf{m})=\operatorname{supp} \mathbf{m}$. By (i), w $\in \mathcal{M}$ and supp $\mathbf{w} \cap F(\mathbf{m})=\emptyset$ imply $\mathbf{m}+\mathbf{w} \in \mathcal{M}$.

Remark 2.23. Let $F I N^{\ell}=\{\mathbf{m} \in F I N(\mathbb{N}): \operatorname{supp} \mathbf{m} \cap[1, \ell]=\emptyset\}$. It is not hard to show from the above Proposition 2.22 that, if $\mathcal{M}$ is a strongly recurrent label $\mathcal{M}$, then $\bigcap_{\ell} \overline{\mathcal{M} \cap F I N^{\ell}}=I S O(\mathcal{M})$, where the closure is taken in $\beta F I N(\mathbb{N})$.

The following directly illustrates the relationship between recurrence and the failure of the finite type condition.

Corollary 2.24. If $\left\{\mathbf{m}^{i}\right\}$ is a strictly increasing infinite sequence in a label $\mathcal{M}$ then $\rho \in \mathbb{Z}_{+\infty}^{\mathbb{N}}$ defined by $\rho_{\ell}=\sup _{i}\left\{\mathbf{m}_{\ell}^{i}\right\}$ satisfies $\rho \leq \rho(\mathcal{M})$ and $\mathcal{N}=$ $\langle\rho\rangle=\bigcup_{i}\left\{\left\langle\mathbf{m}^{i}\right\rangle\right\}$ is a recurrent label with $\mathcal{N} \subset \mathcal{M}$ and $\mathbf{m}^{i} \in \mathcal{N}$ for all $i$. If $\mathcal{M}$ is bounded then $\mathcal{N}$ is strongly recurrent.

Proof: Since the sequence is strictly increasing it is clear that

$$
\langle\rho\rangle=\bigcup_{i}\left\{\left\langle\mathbf{m}^{i}\right\rangle\right\} \subset \mathcal{M}
$$

If $\mathcal{M}$ is bounded, then $\langle\rho\rangle$ is bounded and infinite and so is strongly recurrent by Proposition 2.22 (e).

Corollary 2.25. (a) For a label $\mathcal{M}$ the following are equivalent: 
(i) $\mathcal{M}$ is of finite type.

(ii) The only recurrent label which is a subset of $\mathcal{M}$ is $\emptyset$.

(iii) The only recurrent label which is contained in $\Theta(\mathcal{M})$ is $\emptyset$.

(iv) $Q(\mathcal{M})=\emptyset$ for every idempotent $Q$ in $\beta^{*} F I N(\mathbb{N})$.

(b) For a label $\mathcal{M}, \quad \max \mathcal{M}=\emptyset$ iff $\mathcal{M}$ is the union of the recurrent labels contained in $\mathcal{M}$. Thus:

$$
\begin{gathered}
\{\mathcal{M}: \text { finite type }\} \subset\{\mathcal{M}: \max \mathcal{M} \neq \emptyset\}= \\
\{\mathcal{M}: \text { not a union of recurrent labels }\} \subset\{\mathcal{M}: \text { not recurrent }\} .
\end{gathered}
$$

Proof: (a): (i) $\Rightarrow$ (iv): Part (c) of Proposition 2.20.

(iv) $\Rightarrow$ (ii): If $Q(\mathcal{M})=\emptyset$ then $Q(\mathcal{N})=\emptyset$ for all $\mathcal{N} \subset \mathcal{M}$.

(ii) $\Leftrightarrow$ (iii): Proposition 2.20 (d).

(ii) $\Rightarrow$ (i): If $\mathcal{M}$ is not of finite type then it contains a strictly increasing infinite sequence and so by Corollary 2.24 it contains a recurrent label.

(b): If $\mathbf{m} \in \max \mathcal{M}$ and $\mathbf{m} \in \mathcal{N} \subset \mathcal{M}$ then $\mathbf{m} \in \max \mathcal{N}$ and so $\mathcal{N}$ is not recurrent. That is, $\max \mathcal{M}$ is disjoint from all nonempty recurrent labels contained in $\mathcal{M}$.

If $\max \mathcal{M}=\emptyset$ and $\mathbf{m} \in \mathcal{M}$ then inductively we can define a strictly increasing sequence $\left\{\mathbf{m}^{i}\right\}$ in $\mathcal{M}$ with $\mathbf{m}=\mathbf{m}^{1}$. By Corollary 2.24 there is a recurrent label $\mathcal{N} \subset \mathcal{M}$ with $\mathbf{m} \in \mathcal{N}$.

Corollary 2.26. Assume $\mathcal{M}$ is a nonempty, bounded label. If $\mathcal{M}$ is a recurrent label then $\Theta(\mathcal{M})$ is a Cantor set. If $\mathcal{M}$ is not of finite type, then $\Theta(\mathcal{M})$ is uncountable.

Proof: If $\mathcal{M}$ is a nonempty recurrent label then for some sequence $\left\{\mathbf{r}^{i}\right\}$ of positive elements of $\mathcal{M},\left\{\mathcal{M}-\mathbf{r}^{i}\right\}$ converges to $\mathcal{M}$. Since $\mathcal{M}$ is bounded, each of the $\mathcal{M}-\mathbf{r}^{i}$ is a proper subset of $\mathcal{M}$ and each lies in $\Theta(\mathcal{M})$. It follows that $\mathcal{M}$ is not an isolated point of $\Theta(\mathcal{M})$. For each $\mathbf{r} \in \mathcal{M}$, the label $\mathcal{M}-\mathbf{r}$ is nonempty and bounded and it is recurrent by Proposition 2.20(e). Hence, $\mathcal{M}-\mathbf{r}$ is not isolated in $\Theta(\mathcal{M}-\mathbf{r}) \subset \Theta(\mathcal{M})$. It follows that $\{\mathcal{M}-\mathbf{r}: \mathbf{r} \in \mathcal{M}\}$ is a dense subset of $\Theta(\mathcal{M})$ no point of which is isolated and so no point of $\Theta(\mathcal{M})$ is isolated. On the other hand, $\Theta(\mathcal{M})$ is a nonempty, compact, ultra-metric space. It follows that it is a Cantor set.

If $\mathcal{M}$ is not of finite type then there exists a nonempty recurrent $\mathcal{N} \in \Theta(\mathcal{M})$ by Corollary 2.25(a). Hence, $\Theta(\mathcal{M})$ contains the Cantor set $\Theta(\mathcal{N})$.

Corollary 2.27. For any label $\mathcal{M}, \mathcal{M}$ is the only $F I N(\mathbb{N})$-transitive point in $\Theta(\mathcal{M})$. 
Proof: Suppose $\mathcal{N} \in \Theta(\mathcal{M})$ is a transitive point. Then there are $P, Q \in$ $\beta F I N(\mathbb{N})$ with $P(\mathcal{M})=\mathcal{N}$ and $Q(\mathcal{N})=\mathcal{M}$. Thus $Q P \in I S O(\mathcal{M})$ and it follows, by Proposition 2.20(a), that $P \in I S O(\mathcal{M})$ and so $\mathcal{N}=\mathcal{M}$.

Remark 2.28. Note the sharp contrast with the case where the acting semigroup is a group. In fact, for a dynamical system $(X, \Gamma)$, where $X$ is a compact metric space and $\Gamma$ is a group, the existence of one dense orbit implies that the set $X_{t r}$ of transitive points forms a dense $G_{\delta}$ subset of $X$. See Proposition 1.1 .

Example 2.29. It can happen that $\Theta(\mathcal{M})$ is uncountable even with $\mathcal{M}$ of finite type.

Define a bijection $w \mapsto \ell_{w}$, from the set of finite words on the alphabet $\{0,1\}$ onto $\mathbb{N}$. For $x \in\{0,1\}^{\mathbb{N}}$ let $w_{k}(x)$ be the initial word of length $k$ in $x$, that is, $w_{k}(x)=x_{1} x_{2} \ldots x_{k}$, for $k \in \mathbb{N}$.

Let

$$
\begin{gathered}
M_{x}=\left\{\ell_{w_{k}(x)}: k \in \mathbb{N}\right\} \\
\mathcal{M}_{x}=\{\mathbf{0}\} \cup\left\{\chi(\ell): \ell \in M_{x}\right\}, \\
\mathcal{M}_{x}^{(2)}=\mathcal{M}_{x} \oplus \mathcal{M}_{x}=\left\langle\left\{\chi\left(\ell^{1}\right)+\chi\left(\ell^{2}\right): \ell^{1}, \ell^{2} \in M_{x}\right\}\right\rangle, \\
\mathcal{M}=\bigcup\left\{\mathcal{M}_{x}^{(2)}: x \in\{0,1\}^{\mathbb{N}}\right\} .
\end{gathered}
$$

Since $\rho(\mathcal{M}) \leq 2$ and the size of the elements of $\mathcal{M}$ are bounded by 2 , it follows from Lemma 2.4 that $\mathcal{M}$ is a label of finite type. Notice that if $x \neq y$ in $\{0,1\}^{\mathbb{N}}$ then $M_{x} \cap M_{y}$ is finite.

It is easy to see that for each $x \in\{0,1\}^{\mathbb{N}}, \quad \operatorname{LIM}\left\{\mathcal{M}-\chi\left(\ell_{w_{i}(x)}\right)\right\}=\mathcal{M}_{x}$ and it follows that $\Theta(\mathcal{M})$ is uncountable.

If $\Phi$ is a compact, invariant subset of $\mathcal{L} \mathcal{A B}$ then the action of $F I N(\mathbb{N})$ restricts to an action on $\Phi$ as does the Ellis action of $\beta F I N(\mathbb{N})$. The image of the continuous map $\beta F I N(\mathbb{N}) \rightarrow \Phi^{\Phi}$ which extends $\mathbf{r} \mapsto P_{\mathbf{r}}$ is the enveloping semigroup of $\Phi$, denoted $\mathcal{E}(\Phi)$. A map $Q$ on $\Phi$ is an element of $\mathcal{E}(\Phi)$ iff for every finite sequence $\left\{\mathcal{M}^{i}\right\}$ in $\Phi$ and any $N \in \mathbb{N}$ there exists $\mathbf{r} \in F I N(\mathbb{N})$ such that $P_{\mathbf{r}}\left(\mathcal{M}^{i}\right) \cap \mathcal{B}_{N}=Q\left(\mathcal{M}^{i}\right) \cap \mathcal{B}_{N}$ for all $i$.

The adherence semigroup $\mathcal{A}(\Phi)$ is the closure in $\Phi^{\Phi}$ of $\left\{P_{\mathbf{r}}: \mathbf{r}>\mathbf{0}\right\}$, i.e. the image of $\beta^{\prime} F I N(\mathbb{N})$ (see Definition C.9 in Appendix C). It follows that for $\mathcal{M} \in \Phi$

$$
\begin{gathered}
\mathcal{E}(\Phi)=\mathcal{A}(\Phi) \cup\left\{P_{\mathbf{0}}=i d_{\Phi}\right\} \\
\mathcal{E}(\Phi) \cdot \mathcal{M}=\beta F I N(\mathbb{N}) \cdot \mathcal{M}=\Theta(\mathcal{M}), \\
\mathcal{A}(\Phi) \cdot \mathcal{M}=\beta^{\prime} F I N(\mathbb{N}) \cdot \mathcal{M}=\Theta^{\prime}(\mathcal{M}) .
\end{gathered}
$$


If $\Phi_{1} \subset \Phi$ is also closed and invariant then the restriction map defines a continuous, surjective homomorphism from $\mathcal{E}(\Phi)$ to $\mathcal{E}\left(\Phi_{1}\right)$. In particular, every $\mathcal{E}(\Phi)$ is the image of $\mathcal{E}(\mathcal{L} \mathcal{A B})$ via the restriction map.

Proposition 2.30. The enveloping semigroup $\mathcal{E}(\mathcal{L} \mathcal{A B})$ contains a unique minimal idempotent $U$ defined by

$$
U(\mathcal{M})=\left\{\begin{array}{lr}
\emptyset & \text { if } \mathcal{M} \neq F I N(\mathbb{N}), \\
F I N(\mathbb{N}) & \text { if } \mathcal{M}=F I N(\mathbb{N}) .
\end{array}\right.
$$

Furthermore, $P U=U=U P$ for all $P \in \mathcal{E}(\mathcal{L} \mathcal{A B})$.

Proof: The set $I$ of labels not equal to $F I N(\mathbb{N})$ is directed by inclusion for if $\mathbf{r}_{1} \notin \mathcal{M}_{1}$ and $\mathbf{r}_{2} \notin \mathcal{M}_{2}$ then $\mathbf{r}_{1}+\mathbf{r}_{2} \notin \mathcal{M}_{1} \cup \mathcal{M}_{2}$. Choose for each $i \in I$ an element $\mathbf{r}^{i}$ not in the corresponding label. If $\mathcal{M} \neq F I N(\mathbb{N})$ then $\left\{\mathcal{M}-\mathbf{r}^{i}\right\}$ converges to $\emptyset$. Since $F I N(\mathbb{N})-\mathbf{r}=F I N(\mathbb{N})$ for all $\mathbf{r}$ it follows that $P(F I N(\mathbb{N}))=F I N(\mathbb{N})$ for all $P \in \mathcal{E}(\mathcal{L A B})$. Thus, $\left\{P_{\mathbf{r}^{i}}\right\}$ converges to $U$. Clearly, $P U=U=U P$ from which it follows that $\{U\}$ is the unique minimal subset of $\mathcal{E}(\mathcal{L} \mathcal{A B})$.

No point of $\beta^{*} F I N(\mathbb{N})$ is the limit of a sequence in $F I N(\mathbb{N})$ (see Corollary C.2). On the other hand, $\mathcal{E}(\mathcal{L} \mathcal{A B})$ has interesting points which are limits of sequences in $F I N(\mathbb{N})$.

Example 2.31. For any $\mathbf{r}>0$ the sequence $\left\{P_{n \mathbf{r}}: n \in \mathbb{N}\right\}$ converges in the enveloping semigroup $\mathcal{E}(\mathcal{L} \mathcal{A B})$ to an idempotent $Q_{\mathbf{r}}$ with $Q_{\mathbf{r}}(\mathcal{M})=\{\mathbf{m}$ : $\mathbf{m}+n \mathbf{r} \in \mathcal{M}$ for all $n \in \mathbb{N}\}$.

On the other hand, if $\left\{\mathbf{r}^{i}\right\}$ is a sequence of positive $\mathbb{N}$-vectors such that $L I M S U P\left\{\right.$ supp $\left.\mathbf{r}^{i}\right\}=\emptyset$ then the sequence $\left\{P_{\mathbf{r}^{i}}\right\}$ is not convergent in $\mathcal{E}(\mathcal{L} \mathcal{A B})$. By going to a subsequence we can assume that the supports are pairwise disjoint. Let $\mathcal{M}=\left\langle\left\{\mathbf{r}^{1}+\mathbf{r}^{i}: i \in 2 \mathbb{N}\right\}\right\rangle$. Then $\mathbf{r}^{1} \in P_{\mathbf{r}^{i}}(\mathcal{M})$ for $i$ even but $P_{\mathbf{r}^{i}}(\mathcal{M})=\emptyset$ for odd $i>1$.

We consider convergence of nets of the form $\mathcal{M}-\mathbf{r}^{i}=P_{\mathbf{r}^{i}}(\mathcal{M})$ for a net $\left\{\mathbf{r}^{i}\right\}$ of $\mathbb{N}$-vectors and a fixed label $\mathcal{M}$. Clearly, $\mathbf{m} \in L I M S U P$ iff frequently $\mathbf{m}+\mathbf{r}^{i} \in \mathcal{M}$ and $\mathbf{m} \in L I M I N F$ iff eventually $\mathbf{m}+\mathbf{r}^{i} \in \mathcal{M}$.

Lemma 2.32. Assume that the net $\left\{P_{\mathbf{r}^{i}}(\mathcal{M})=\mathcal{M}-\mathbf{r}^{i}\right\}$ is convergent.

(a) If $\max \mathcal{M} \neq \emptyset$, e.g. if $\mathcal{M}$ is of finite type, then either eventually $\mathbf{r}^{i}=\mathbf{0}$ and $L I M\left\{\mathcal{M}-\mathbf{r}^{i}\right\}=\mathcal{M}=\mathcal{M}-\mathbf{0}$ or eventually $\mathbf{r}^{i}>\mathbf{0}$ and $(\max \mathcal{M}) \cap$ $\operatorname{LIM}\left\{\mathcal{M}-\mathbf{r}^{i}\right\}=\emptyset$.

(b) Either eventually $\mathbf{r}^{i} \in \mathcal{M}$ and $\mathbf{0} \in \operatorname{LIM}\left\{\mathcal{M}-\mathbf{r}^{i}\right\}$ or eventually $\mathbf{r}^{i} \notin \mathcal{M}$ and $L I M\left\{\mathcal{M}-\mathbf{r}^{i}\right\}=\emptyset$.

(c) If $\mathcal{M}-\mathbf{m}_{1}=\mathcal{M}-\mathbf{m}_{2}$ then LIM $\left\{\mathcal{M}-\mathbf{r}^{i}\right\}-\mathbf{m}_{1}=L I M\left\{\mathcal{M}-\mathbf{r}^{i}\right\}-\mathbf{m}_{2}$. 
(d) If there exists $\mathbf{r}$ such that frequently, $\mathcal{M}-\mathbf{r}^{i}=\mathcal{M}-\mathbf{r}$ then the limit is $\mathcal{M}-\mathbf{r}$.

Proof: (a) Since $\mathbf{r}>\mathbf{0}$ implies $\max \mathcal{M} \cap(\mathcal{M}-\mathbf{r})=\emptyset$, if $\mathbf{r}^{i}=\mathbf{0}$ frequently then convergence implies that the limit is $\mathcal{M}$ and so any element of $\max \mathcal{M}$ is eventually in $\mathcal{M}-\mathbf{r}^{i}$ which can only happen when $\mathbf{r}^{i}$ is eventually $\mathbf{0}$.

(b) Since $\mathbf{0} \in \mathcal{M}-\mathbf{r}$ iff $\mathbf{r} \in \mathcal{M}$ we see that if $\mathbf{r}^{i} \in \mathcal{M}$ frequently then convergence implies that eventually $\mathbf{0} \in \mathcal{M}-\mathbf{r}^{i}$ and so eventually $\mathbf{r}^{i} \in \mathcal{M}$.

(c) Since $P_{\mathbf{m}_{1}}$ and $P_{\mathbf{m}_{2}}$ are continuous,

$$
\begin{aligned}
& L I M\left\{\mathcal{M}-\mathbf{r}^{i}\right\}-\mathbf{m}_{1}=\operatorname{LIM}\left\{\mathcal{M}-\mathbf{m}_{1}-\mathbf{r}^{i}\right\}= \\
& L I M\left\{\mathcal{M}-\mathbf{m}_{2}-\mathbf{r}^{i}\right\}=L I M\left\{\mathcal{M}-\mathbf{r}^{i}\right\}-\mathbf{m}_{2} .
\end{aligned}
$$

(d) By assumption there is a subnet $\mathbf{r}^{i^{\prime}}$ such that $\mathcal{M}-\mathbf{r}^{i^{\prime}}$ is constant at $\mathcal{M}-\mathbf{r}$ and so converges to $\mathcal{M}-\mathbf{r}$. By the assumption of convergence the limit of the original sequence is $\mathcal{M}-\mathbf{r}$.

For labels $\mathcal{M}_{1}$ and $\mathcal{M}_{2}$ define

$$
\mathcal{M}_{1} \oplus \mathcal{M}_{2}=\left\{\mathbf{m}_{1}+\mathbf{m}_{2}: \mathbf{m}_{1} \in \mathcal{M}_{1}, \mathbf{m}_{2} \in \mathcal{M}_{2}\right\} .
$$

This is empty if either $\mathcal{M}_{1}$ or $\mathcal{M}_{2}$ is empty. It follows that

$$
\rho\left(\mathcal{M}_{1} \oplus \mathcal{M}_{2}\right)=\rho\left(\mathcal{M}_{1}\right)+\rho\left(\mathcal{M}_{2}\right) .
$$

and so $\mathcal{M}_{1} \oplus \mathcal{M}_{2}$ is bounded iff both $\mathcal{M}_{1}$ and $\mathcal{M}_{2}$ are bounded.

Observe that for any $\ell, N \in \mathbb{N}$, we have

$$
\begin{gathered}
\left(\mathcal{M}_{1} \oplus \mathcal{M}_{2}\right) \wedge[1, \ell]=\left(\mathcal{M}_{1} \wedge[1, \ell]\right) \oplus\left(\mathcal{M}_{2} \wedge[1, \ell]\right), \\
\left(\mathcal{M}_{1} \oplus \mathcal{M}_{2}\right) \cap \mathcal{B}_{N}=\left[\left(\mathcal{M}_{1} \cap \mathcal{B}_{N}\right) \oplus\left(\mathcal{M}_{2} \cap \mathcal{B}_{N}\right)\right] \cap \mathcal{B}_{N}
\end{gathered}
$$

It follows that the map $\oplus: \mathcal{L} \mathcal{A B} \times \mathcal{L} \mathcal{A B} \rightarrow \mathcal{L} \mathcal{A B}$ is continuous.

Lemma 2.33. (a) If $\mathcal{M}$ is a recurrent label, then $\mathcal{M}_{1} \oplus \mathcal{M}$ is a recurrent label for any label $\mathcal{M}_{1}$. If $\mathcal{M}$ is a strongly recurrent label, then $\mathcal{M}_{1} \oplus \mathcal{M}$ is a strongly recurrent label for any finite label $\mathcal{M}_{1}$.

(b) If $\mathcal{M}$ is any label and $\ell \in \mathbb{N}$ then there exists a recurrent label $\mathcal{M}_{1}$ with $\mathcal{M} \subset \mathcal{M}_{1}$ and $\mathcal{M} \wedge[1, \ell]=\mathcal{M}_{1} \wedge[1, \ell]$. There exists a strongly recurrent label $\mathcal{M}_{2}$ with $\mathcal{M} \wedge[1, \ell]=\mathcal{M}_{2} \wedge[1, \ell]$. If $\mathcal{M}$ is bounded then $\mathcal{M}_{1}$ and $\mathcal{M}_{2}$ can be chosen bounded.

Proof: (a) Clearly $\mathcal{M}_{1} \oplus(\mathcal{M}-\mathbf{r}) \subset\left(\mathcal{M}_{1} \oplus \mathcal{M}\right)-\mathbf{r}$ for any $\mathbf{r} \in \mathcal{M}$. Hence, if $\left\{\mathcal{M}-\mathbf{r}^{i}\right\} \rightarrow \mathcal{M}$ then $\left\{\left(\mathcal{M}_{1} \oplus \mathcal{M}\right)-\mathbf{r}^{i}\right\} \rightarrow \mathcal{M}_{1} \oplus \mathcal{M}$ and so $\mathcal{M}_{1} \oplus \mathcal{M}$ is recurrent.

If $\mathbf{m}_{1} \in \mathcal{M}_{1}$ and $\mathbf{m} \in \mathcal{M}$ we let $F\left(\mathbf{m}_{1}+\mathbf{m}\right)=\left(\bigcup \operatorname{Supp} \mathcal{M}_{1}\right) \cup F(\mathbf{m})$ (see Definition 2.21). If $\mathbf{r}_{1}+\mathbf{r}$ has support disjoint from this set then $\mathbf{r}_{1}=0$ and $\mathbf{r}+\mathbf{m} \in \mathcal{M}$. Hence, $\mathbf{r}_{1}+\mathbf{r}+\mathbf{m} \in \mathcal{M}_{1} \oplus \mathcal{M}$. 
(b) Begin with a bounded, strongly recurrent label $\mathcal{N}$ with $\mathcal{N} \wedge[1, \ell]=0$. For example, $\mathcal{N}=\langle\chi([\ell+1, \infty))\rangle$ is strongly recurrent by Proposition 2.22. Then $\mathcal{M}_{1}=\mathcal{M} \oplus \mathcal{N}$ is a recurrent label and $\mathcal{M}_{2}=(\mathcal{M} \wedge[1, \ell]) \oplus \mathcal{N}$ is a strongly recurrent label with $\mathcal{M} \wedge[1, \ell]=\mathcal{M}_{1} \wedge[1, \ell]=\mathcal{M}_{2} \wedge[1, \ell]$.

Example 2.34. Let $L$ be an infinite set disjoint from $\bigcup$ Supp $\mathcal{M}$. Let $\mathcal{M}_{1}=$ $\{\emptyset\} \cup\{\chi(\ell): \ell \in L\}$. If $\mathcal{M}$ is strongly recurrent then $\mathcal{M}_{1} \oplus \mathcal{M}$ is recurrent by (a) but it is not strongly recurrent.

Proposition 2.35. The set $R E C U R$ of recurrent labels is a dense, $G_{\delta}$ subset of $\mathcal{L} \mathcal{A B}$.

Proof: For any $N \in \mathbb{N}$ and $\mathbf{r} \in F I N(\mathbb{N})$, the set $\left\{\mathcal{M}: \mathcal{M} \cap \mathcal{B}_{N}=\right.$ $\left.P_{\mathbf{r}}(\mathcal{M}) \cap \mathcal{B}_{N}\right\}$ is a clopen subset of $\mathcal{L} \mathcal{A B}$ by Lemma 2.7 (c) and continuity of the map $P_{\mathbf{r}}$.

Clearly,

$$
R E C U R=\bigcap_{N \in \mathbb{N}} \bigcup_{\mathbf{r} \in F I N}\left\{\mathcal{M}: \mathcal{M} \cap \mathcal{B}_{N}=P_{\mathbf{r}}(\mathcal{M}) \cap \mathcal{B}_{N}\right\}
$$

and so $R E C U R$ is a $G_{\delta}$ subset of $\mathcal{L} \mathcal{A B}$. It is dense by Lemma 2.33 (b).

Thus, the labels of finite type, upon which we focus most of our attention, comprise a subset of first category in $\mathcal{L} \mathcal{A B}$. Since the finite labels are dense, the labels of finite type are dense. Thus, the set of recurrent labels has empty interior. As it is the intersection of two dense $G_{\delta}$ sets, the set of bounded, recurrent labels is a dense $G_{\delta}$ set.

\section{LABELED SUBSHIFTS}

\subsection{Integer expansions.}

Let $e \in \mathbb{N}$ and $b=2 e+1$ so that $b$ is an odd number greater than 1 . Define $k: \mathbb{N} \rightarrow \mathbb{N}$ by $k(n)=b^{n-1}$. The symmetric b-expansion of an integer $t$ is the $\operatorname{sum} \Sigma_{n \in \mathbb{N}} \epsilon_{n}(t) k(n)=t$ such that:

- $\left|\epsilon_{n}(t)\right| \leq e$ for all $n \in \mathbb{N}$.

- $\epsilon_{n}(t)=0$ for all but finitely many $n$. 
Proposition 3.1. Every integer $t \in \mathbb{Z}$ has a unique symmetric b-expansion and $\epsilon_{n}(-t)=-\epsilon_{n}(t)$ for all $t \in \mathbb{Z}, n \in \mathbb{N}$.

Proof: By the Euclidean Algorithm every integer $t$ can be expressed uniquely as $\epsilon+b s$ with $|\epsilon| \leq e$. It follows by induction that every integer $t$ with $|t|<\frac{1}{2}\left(b^{k}-1\right)$ has an expansion with $\epsilon_{n}=0$ for $n \geq k$. There are $b^{k}$ such integers and the same number of expansions. So by the Pigeonhole Principle the expansions are unique.

Since $-t=\Sigma_{n \in \mathbb{N}}-\epsilon_{n}(t) k(n)$ and the expansions are unique, it follows that $-\epsilon_{n}(t)=\epsilon_{n}(-t)$.

Definition 3.2. For $t \in \mathbb{Z} \backslash\{0\}$ let

$$
\begin{aligned}
& n_{*}(t)=\min \left\{n \in \mathbb{N}: \epsilon_{n}(t) \neq 0\right\}, \\
& n^{*}(t)=\max \left\{n \in \mathbb{N}: \epsilon_{n}(t) \neq 0\right\} .
\end{aligned}
$$

Thus, $n_{*}(t)$ is the smallest non-zero place in the expansion of $t$, and $n^{*}(t)$ is the largest. From Proposition $3.1 n_{*}(-t)=n_{*}(t)$ and $n^{*}(-t)=n^{*}(t)$. By convention, we define $n_{*}(0)=\infty$ and $n^{*}(0)=0$. Observe that for $k \in \mathbb{N}$,

$$
n_{*}(t)>k \quad \Longleftrightarrow \quad t \equiv 0\left(\bmod b^{k}\right) \text {. }
$$

Define the function $s k: \mathbb{Z}_{+} \rightarrow \mathbb{Z}_{+}$by

$$
s k(n)=\sum_{i=1}^{n-1} k(i),
$$

with $s k(1)=s k(0)=0$, the empty sum.

We extend $k$ to an odd function on $\mathbb{Z}$ so that $k(-j)=-k(j)$ for all $j \in \mathbb{Z}$ and so, in particular, $k(0)=0$. Clearly, $|k(j)|=k(|j|)$ for all $j \in \mathbb{Z}$.

For integers $a, b$ we will denote by $[a \pm b]$ the interval $[a-|b|, a+|b|]$ in $\mathbb{Z}$. When $a=0$ we will write $[ \pm b]$ for the interval $[-|b|,+|b|]$.

Clearly, for $n \in \mathbb{N}$

$$
\begin{aligned}
& \quad k(n)=1+(b-1) s k(n), \\
& \text { and so } \quad s k(n)<\frac{1}{b-1} k(n) .
\end{aligned}
$$

Also, for $n \in \mathbb{N}$ :

$$
k(n+1)-k(n)=(b-1) k(n) .
$$

Lemma 3.3. (a) For $t \in \mathbb{Z} \backslash\{0\}, t>0$ iff $\epsilon_{n^{*}}(t)>0$ and

$$
\frac{b-1}{2} \operatorname{sk}\left(n^{*}(t)+1\right) \geq|t| \geq \frac{b-1}{2} \operatorname{sk}\left(n^{*}(t)\right) .
$$

(b) The sequence $\left\{\left[k(n) \pm \frac{b-1}{2} s k(|n|)\right]: n \in \mathbb{Z}\right\}$ is a pairwise disjoint bi-infinite sequence of intervals in $\mathbb{Z}$. 
(c) For $t, s \in \mathbb{Z}$, if $\left|\epsilon_{n}(t)+\epsilon_{n}(s)\right| \leq$ for all $n \in \mathbb{N}$, then $\epsilon_{n}(s+t)=$ $\epsilon_{n}(t)+\epsilon_{n}(s)$ for all $n \in \mathbb{N}$.

Proof: Recall that $\frac{b-1}{2}=e$, a positive integer.

(a) If $\epsilon_{n^{*}(t)}>0$ then $t \geq k\left(n^{*}(t)\right)-e s k\left(n^{*}(t)\right)$ which is positive by (3.4). $\epsilon_{n^{*}(t)}<0$ then $\epsilon\left(n^{*}(-t)\right)=-\epsilon\left(n^{*}(t)\right)>0$ and so $-t>0$.

In any case, for any non-zero $t$ we have $e\left(k\left(n^{*}\right)+s k\left(n^{*}\right)\right) \geq|t| \geq k\left(n^{*}\right)-$ $\operatorname{esk}\left(n^{*}\right)$ and the inequalities of (3.6) follow from the definition of $s k\left(n^{*}+1\right)$ and (3.4).

(b) If $|n|=0,1$ then $s k(|n|)=0$ and the intervals are the singletons $\{k(n)\}$. For $n>1,(3.4)$ and $e \geq 1$ imply that

$$
k(n)-\operatorname{esk}(n)>\operatorname{esk}(n) \geq k(n-1)+\operatorname{esk}(n-1) .
$$

(c) This is clear from the uniqueness of the symmetric expansion. The assumption implies that there are no "carries" in any place when $t$ and $s$ are added.

We now fix $e>1$ and so $b=2 e+1 \geq 5$. We will frequently use the observation:

$$
n \geq 2 \quad \Longrightarrow \quad k(n) \geq \frac{b}{2} n>2 n .
$$

Let $I P(k)$ denote the set of sums of finite subsets of the image set $k(\mathbb{Z}) \subset$ $\mathbb{Z}$ and let $I P_{+}(k)$ denote the set of sums of finite subsets of the image set $k\left(\mathbb{Z}_{+}\right) \subset \mathbb{Z}_{+}$. It is clear that $I P(k)$ consists of those integers $t=\Sigma_{n \in \mathbb{N}} \epsilon_{n} k(n)$ such that every nonzero $\epsilon_{n}= \pm 1$. That is, we exclude those integers $t$ such that some $\epsilon_{n}$ has absolute value equal to $2,3, \ldots e$. In $I P_{+}(k)$ every $\epsilon_{n}$ is zero or one. Notice that Lemma 3.3 (c) applies to any $t, s \in I P(k)$.

For $t \in I P(k)$ we have $t=\Sigma_{n \in \mathbb{N}} k\left(\epsilon_{n} n\right)$ and so for such $t$ we describe the expansion a slightly different way, listing only the nonzero terms and absorbing the sign into the index.

Definition 3.4. (a) For $t \in I P(k)$ we write

$$
t=k\left(j_{1}\right)+k\left(j_{2}\right)+\cdots+k\left(j_{r}\right) .
$$

with $j_{1}, \ldots, j_{r} \in \mathbb{Z}$ such that $\left|j_{i}\right|>\left|j_{i+1}\right|>0$ for $i=1, \ldots, r-1$. We will call $j_{1}, \ldots, j_{r}$ the expansion of $t \in I P(k)$, and we call $r=r(t) \geq 0$ the length of the expansion.

$0 \in I P(k)$ has the empty expansion with length 0 .

By convention, if $i>r(t)$ then we write $j_{i}(t)=0$.

(b) For $0 \leq \tilde{r} \leq r$ the $\tilde{r}$ truncation is the element $\tilde{t} \in I P(k)$ with expansion $j_{1}, \ldots, j_{\tilde{r}}$ so that

$$
\tilde{t}=k\left(j_{1}\right)+k\left(j_{2}\right)+\cdots+k\left(j_{\tilde{r}}\right)
$$


The sequence $j_{\tilde{r}+1}, \ldots, j_{r}$ is the expansion for the $\tilde{r}$ residual $t-\tilde{t}$ with length $r-\tilde{r}$. We call $\tilde{t}$ a truncation of $t$ and $t$ an extension of $\tilde{t}$.

When $\tilde{r}=0$ the truncation $\tilde{t}=0$ with the empty expansion. When $\tilde{r}=r$ the truncation $\tilde{t}=t$ with residual 0 .

Comparing the alternative notations we have for $t \in I P(k) \backslash\{0\}$

$$
\left|j_{1}(t)\right|=n^{*}(t), \quad\left|j_{r(t)}(t)\right|=n_{*}(t) .
$$

Lemma 3.5. (a) Let $t \in I P(k) \backslash\{0\}$. The integer $t$ has a unique expansion $j_{1}, \ldots, j_{r}$ with $r \geq 1$ and $t$ has the same sign as $k\left(j_{1}\right)$. Furthermore,

$$
\begin{array}{r}
\left|t-k\left(j_{1}(t)\right)\right| \leq \sum_{i=2}^{r}\left|k\left(j_{i}(t)\right)\right| \leq s k\left(\left|j_{1}(t)\right|\right), \\
\frac{b-2}{b-1}\left|k\left(j_{1}(t)\right)\right|<|t|<\frac{b}{b-1}\left|k\left(j_{1}(t)\right)\right|,
\end{array}
$$

(b) If $\tilde{t}$ is the $\tilde{r}$ truncation of then

$$
\frac{b-2}{b-1}\left|k\left(j_{\tilde{r}+1}(t)\right)\right| \leq|t-\tilde{t}| \leq \frac{b}{b-1}\left|k\left(j_{\tilde{r}+1}(t)\right)\right| .
$$

(c) If $t, s$ are distinct integers and $\hat{r}$ is the smallest positive integer $p$ such that $j_{p}(t) \neq j_{p}(s)$, then

$$
|t-s|>\frac{b-3}{b-1} \cdot \max \left(\left|k\left(j_{\hat{r}}(t)\right)\right|,\left|k\left(j_{\hat{r}}(s)\right)\right|\right) .
$$

Proof: (a): The uniqueness of the expansion is Proposition 3.1. That $t$ and $j_{1}(t)$ have the same sign follows Lemma 3.3.

Because the sequence $\left\{\left|j_{i}\right|: i=2, \ldots, r\right\}$ consists of distinct positive integers all less than $\left|j_{1}\right|$ the first line of (3.10) is clear. The second follows from (3.4).

(b): If $r=\tilde{r}$ then $t-\tilde{t}=0=j_{\tilde{r}+1}(t)$ and so (3.11) is clear.

If $r>\tilde{r}$ then $t-\tilde{t}$ is the residual whose expansion begins with $j_{\tilde{r}+1}(t)$. So (3.11) follows from (3.10).

(c): Applying Lemma 3.3 we see that $t-s$ has a symmetric $b$ expansion with $\left|\epsilon_{n}\right| \leq 2$ for all $n$ and with $n^{*}=n^{*}(t-s)=\max \left(\left|j_{\hat{r}}(t)\right|,\left|j_{\hat{r}}(s)\right|\right)$. It follows from (3.4) that $|t-s| \geq k\left(n^{*}\right)-2 s k\left(n^{*}\right) \geq\left(1-\frac{2}{b-1}\right) k\left(n^{*}\right)$.

Corollary 3.6. If $s, t \in I P(k)$ then $s$ is an extension of $t$ iff

$$
|t-s| \leq \frac{b-3}{b-1}\left|k\left(j_{r}(t)\right)\right|
$$


Proof: The result is clear if $t=s$ and so we can assume $s, t$ are distinct. If $s$ is an extension of $t$ then $t$ is the truncation of $s$ at $r$. By (3.11) $|t-s| \leq$ $\frac{b}{b-1} k\left(\left|j_{r+1}(s)\right|\right) \leq \frac{1}{b-1} k\left(\left|j_{r}(t)\right|\right)$ since $\left|j_{r+1}(s)\right|<\left|j_{r}(s)\right|$ and $j_{r}(s)=j_{r}(t)$. Then (3.13) follows because $b-3 \geq 1$.

On the other hand, if $|t-s| \leq \frac{b-3}{b-1}\left|k\left(j_{r}(t)\right)\right|$ then, in the notation of Lemma $3.5(\mathrm{c})$ it must be true that $\max \left(\left|j_{\hat{r}}(t)\right|,\left|j_{\hat{r}}(s)\right|\right)<\left|j_{r}(t)\right|$. That is, $\hat{r}>r$ and so equals $r+1$ with $j_{\hat{r}}(t)=0$. Thus, $j_{i}(t)=j_{i}(s)$ for $i=1, \ldots, r(t)$. That is, $t$ is a truncation of $s$.

For a subset $A$ of $\mathbb{Z}$ the upper Banach density of $A$ is

$$
\limsup _{\# I \rightarrow \infty} \frac{\#(I \cap A)}{\# I}
$$

as $I$ varies over finite intervals in $\mathbb{Z}$. When the limsup is zero, or, equivalently, when the limit exists and equals zero, we say that $A$ has Banach density zero.

Theorem 3.7. The subset IP $(k)$ of $\mathbb{Z}$ has Banach density zero.

Proof: Given an interval $I \subset \mathbb{Z}$ in with $N=\# I$, if $t, s$ are distinct points in $I$ then $0<|t-s|<N$. Now assume that $t, s \in I P(k)$ and apply Lemma 3.5. Let $\hat{r}$ be the smallest positive integer such that $j_{\hat{r}}(t) \neq j_{\hat{r}}(s)$. Since $b \geq 5$, $\frac{b-3}{b-1} \geq \frac{1}{2}$ and so Lemma 3.5(c) implies that $2 N>\max \left(k\left(\left|j_{\hat{r}}(t)\right|\right), k\left(\left|j_{\hat{r}}(s)\right|\right)\right)$. Thus, the terms in the expansions of $t$ and $s$ agree except for terms $k(j)$ with $|k(j)|<2 N$. Call these the variable $|j|$ 's.

Since $|k(j)|=b^{|j|-1}$ and $b \geq 5$, we have $5^{|j|-1}<2 N$. So the number of variable $|j|$ 's is bounded by $1+\log _{5}(2 N)=\log _{5}(10 N)$. For each such $j, k(|j|)$ can occur with coefficient $-1,0$ or +1 in the symmetric $b$ expansion of $t \in I$. Consequently, $\#(I \cap I P(k))$ is bounded by $3^{\log _{5}(10 N)}=(10 N)^{\log _{5}(3)}$. Since $0<\log _{5}(3)<1$, it follows that $\frac{(10 N)^{\log _{5}(3)}}{N} \rightarrow 0$ as $N \rightarrow \infty$.

Hence,

$$
\lim _{\# I \rightarrow \infty} \frac{\#(I \cap I P(k))}{\# I}=0 .
$$

\subsection{Labeled integers.}

We now fix a partition of $\mathbb{N}$ by an infinite sequence

$$
\mathcal{D}=\left\{D_{\ell}: \ell \in \mathbb{N}\right\}
$$


with $\# D_{\ell}=\infty$ for every $\ell \in \mathbb{N}$, numbered so that $\min D_{\ell}<\min D_{\ell+1}$. For example, if $p_{1}=2, p_{2}, \ldots$ numbers the primes in $\mathbb{N}$ is ascending order we can let $D_{1}=\{1\} \cup 2 \mathbb{N}$ and for $\ell>1$ let $D_{\ell}$ consist of those integers $n$ with $p_{\ell}$ the smallest prime which divides $n$.

In an case, $\min D_{1}=1$ and $\min D_{\ell} \geq \ell$. We then let $Q(\ell, i)$ be the $i^{\text {th }}$ smallest member of $D_{\ell}$. Thus, $Q: \mathbb{N} \times \mathbb{N} \rightarrow \mathbb{N}$ is a bijection such that $\ell \mapsto Q(\ell, 1)$ is increasing and for each $\ell \in \mathbb{N}, i \mapsto Q(\ell, i)$ is increasing with image $D_{\ell}$.

The support map $n \mapsto \ell(n)$ associates to each $n \in \mathbb{N}$ the member of $\mathcal{D}$ which contains it, so that $n \in D_{\ell(n)}$. The map is the composition of $Q^{-1}$ with the projection onto the first coordinate.

Definition 3.8. (a) If $j_{1}, \ldots, j_{r} \in \mathbb{Z}$ is an expansion for $t \in I P(k)$, so that

$$
t=k\left(j_{1}\right)+k\left(j_{2}\right)+\cdots+k\left(j_{r}\right),
$$

then the length vector for the expansion is the $\mathbb{N}$-vector

$$
\mathbf{r}=\mathbf{r}(t)=\chi\left(\ell\left(\left|j_{1}\right|\right)\right)+\chi\left(\ell\left(\left|j_{2}\right|\right)\right)+\cdots+\chi\left(\ell\left(\left|j_{r}\right|\right)\right)
$$

so that $|\mathbf{r}|$ is the length $r=r(t)$.

$0 \in I P_{+}(k) \subset I P(k)$ has the empty expansion with length 0 and length vector $\mathbf{0 .}$

Thus, $\mathbf{r}(t)_{\ell}=\#\left\{i:\left|j_{i}\right| \in D_{\ell}\right\}$ in the expansion $j_{1}, \ldots, j_{r}$ for $t$.

If $0 \leq \tilde{r} \leq r$, then the $\tilde{r}$ truncation, $\tilde{t}$, has length vector $\tilde{\mathbf{r}}=\mathbf{r}(\tilde{t})=$ $\chi\left(\ell\left(j_{1}\right)\right)+\chi\left(\ell\left(j_{2}\right)\right)+\cdots+\chi\left(\ell\left(j_{\tilde{r}}\right)\right)$, so that $|\tilde{\mathbf{r}}|=\tilde{r}$. The residual $t-\tilde{t}$ has length vector $\mathbf{r}-\tilde{\mathbf{r}} \geq \mathbf{0}$.

Definition 3.9. For a label $\mathcal{M}$ define $A[\mathcal{M}] \subset \mathbb{Z}$ to consist of those $t \in I P(k)$ which have length vector $\mathbf{r}(t) \in \mathcal{M}$. Define $A_{+}[\mathcal{M}]=A[\mathcal{M}] \cap I P_{+}(k)$.

If $\mathcal{M}=\emptyset$ then $A_{+}[\mathcal{M}]=A[\mathcal{M}]=\emptyset$. Otherwise $0 \in A_{+}[\mathcal{M}] \subset A[\mathcal{M}]$ since $0 \in \mathcal{M}$.

Proposition 3.10. Assume $t \in I P(k)$ with expansion length $r$. For any positive integer $N$,

$N<\left|j_{r}(t)\right| \Longrightarrow[t \pm N] \cap A[\mathcal{M}]=t+([ \pm N] \cap A[\mathcal{M}-\mathbf{r}(t)])$,

and the elements of $[t \pm N] \cap A[\mathcal{M}]$ are extensions of $t$ in $A[\mathcal{M}]$. In particular, this set is nonempty iff $t \in A[\mathcal{M}]$.

If, in addition, $t \in I P_{+}(k)$ then

$$
[t \pm N] \cap A_{+}[\mathcal{M}]=t+\left([ \pm N] \cap A_{+}[\mathcal{M}-\mathbf{r}(t)]\right)
$$


and the elements of $[t \pm N] \cap A_{+}[\mathcal{M}]$ are extensions of $t$ in $A_{+}[\mathcal{M}]$. In particular, this set is nonempty iff $t \in A_{+}[\mathcal{M}]$.

Proof: If $N<\left|j_{r}(t)\right|$ then $2 \leq\left|j_{r}(t)\right|$ and so (3.8) implies

$$
N<\left|j_{r}(t)\right| \leq \frac{2}{b} k\left(\left|j_{r}(t)\right|\right)<\frac{b-3}{b-1} k\left(\left|j_{r}(t)\right|\right) .
$$

So if $s \in[t \pm N] \cap A[\mathcal{M}]$, then by Corollary $3.6 s$ is an extension of $t$ and $\mathbf{r}(s) \in \mathcal{M}$. Because $t$ is a truncation of $s$ with residual $s-t$ we have $\mathbf{r}(s)=\mathbf{r}(t)+\mathbf{r}(s-t)$ so $s-t \in A[\mathcal{N}-\mathbf{r}(t)]$.

Furthermore, if $s \in[t \pm N] \cap A_{+}[\mathcal{M}]$, i.e. in addition, $s \in I P_{+}(k)$, then since $t$ is a truncation of $s$ with residual $s-t$ we have $t, s-t \in I P_{+}(k)$.

On the other hand, if $u=k\left(j_{1}(u)\right)+\cdots+k\left(j_{p}(u)\right) \in[ \pm N] \cap A[\mathcal{M}-\mathbf{r}(t)]$ with length $p$ then $\mathbf{r}(u)+\mathbf{r}(t) \in \mathcal{M}$.

Furthermore, by (3.10) applied to $u,|u| \leq N$ implies $\left|k\left(j_{1}(u)\right)\right|<2|u| \leq$ $2 N<2\left|j_{r}(t)\right|$.

If $\left|j_{1}(u)\right|=1$ then $\left|j_{1}(u)\right|=1<\left|j_{r}(t)\right|$. Otherwise, (3.8) implies $\left|j_{1}(u)\right| \leq$ $\frac{1}{2} k\left(\left|j_{1}(u)\right|\right)<\left|j_{r}(t)\right|$.

Either way, it follows that

$$
j_{1}(t), \ldots, j_{r}(t), j_{1}(u), \ldots, j_{p}(u)
$$

is the expansion for $t+u$ with length $r+p$ and length vector $\mathbf{r}(t)+\mathbf{r}(u)$. Hence, $t+u \in[t \pm N] \cap A[\mathcal{M}]$.

Furthermore if $u, t \in I P_{+}(k)$ then $t+u \in I P_{+}(k)$.

Lemma 3.11. If $t \in I P(k)$ with $|t|<\min D_{\ell}$, then $\mathbf{r}(t)_{\ell}=0$.

Proof: We may assume $t \neq 0$. So $1 \leq|t|<\min D_{\ell}$ and $\min D_{1}=1$ imply $1<\ell$.

If $\left|j_{1}(t)\right|=1$ then $\left|j_{1}(t)\right|<\ell \leq \min D_{\ell}$.

If $\left|j_{1}(t)\right| \geq 2$ then (3.8) and (3.10) imply

$$
\left|j_{1}(t)\right| \leq \frac{1}{2}\left|k\left(j_{1}(t)\right)\right| \leq \frac{b-1}{2(b-2)}|t|<\min D_{\ell},
$$

since $\frac{b-1}{2(b-2)}<1$.

In either case, $\left|j_{1}(t)\right|<\min D_{\ell}$ implies that $\left|j_{i}(t)\right| \notin D_{\ell}$ for $i=1, \ldots, r(t)$.

Proposition 3.12. For any label $\mathcal{M}$ and integer $N \geq 2$

$$
\begin{aligned}
{[ \pm N] \cap A[\mathcal{M}] } & =[ \pm N] \cap A\left[\mathcal{M} \cap \mathcal{B}_{N}\right] \\
{[ \pm N] \cap A_{+}[\mathcal{M}] } & =[ \pm N] \cap A_{+}\left[\mathcal{M} \cap \mathcal{B}_{N}\right]
\end{aligned}
$$


Proof: If $N<\ell$ then $N<\min D_{\ell}$. Hence, $|t| \leq N$ implies $\mathbf{r}(t)_{\ell}=0$ by Lemma 3.11. Thus, supp $\mathbf{r}(t) \subset[1, N]$.

If $r=|\mathbf{r}(t)| \geq N$. Then $\left|j_{1}\right|>\left|j_{2}\right|>\cdots>\left|j_{r}\right|>0$ implies $\left|j_{1}\right| \geq r \geq N$. In particular, $\left|j_{1}\right| \geq 2$. From (3.8) we obtain $|t| \geq \frac{b-2}{b-1} k\left(\left|j_{1}\right|\right)>\left|j_{1}\right| \geq N$ since $\frac{2(b-2)}{b-1}>1$. Contrapositively, $|t| \leq N$ implies $|\mathbf{r}(t)|<N$.

It follows that $|t| \leq N$ implies $\mathbf{r}(t) \in \mathcal{B}_{N}$.

If $\mathbf{m}=\mathbf{0}$ then $t=0$ is the unique time with length vector $\mathbf{m}$. For a nonzero $\mathbb{N}$-vector $\mathbf{m}$ there are infinitely many such times.

Lemma 3.13. (a) If $\mathbf{m}$ is a nonzero $\mathbb{N}$-vector so that $|\mathbf{m}|=r>0$ then for any positive integer $N$ there exist $t \in I P_{+}(k)$ such that $\mathbf{r}(t)=\mathbf{m}$. and $j_{r}(t)>N$.

(b) For labels $\mathcal{M}_{1}, \mathcal{M}_{2}$ if $\mathbf{m} \in \mathcal{M}_{1} \backslash \mathcal{M}_{2}$ and $t \in I P(k)$ with $\mathbf{r}(t)=\mathbf{m}$ then $t \in A\left[\mathcal{M}_{1}\right] \backslash A\left[\mathcal{M}_{2}\right]$. In particular, $\mathcal{M}_{1}=\mathcal{M}_{2}$ iff $A\left[\mathcal{M}_{1}\right]=A\left[\mathcal{M}_{2}\right]$ and iff $A_{+}\left[\mathcal{M}_{1}\right]=A_{+}\left[\mathcal{M}_{2}\right]$.

(c) For any $N \in \mathbb{N}$ there exists $M \in \mathbb{N}$ such that for all labels $\mathcal{M}_{1}, \mathcal{M}_{2}$

$$
\begin{gathered}
{[ \pm M] \cap A\left[\mathcal{M}_{1}\right]=[ \pm M] \cap A\left[\mathcal{M}_{2}\right] \quad \Longrightarrow} \\
{[ \pm M] \cap A_{+}\left[\mathcal{M}_{1}\right]=[ \pm M] \cap A_{+}\left[\mathcal{M}_{2}\right]} \\
\Longrightarrow \quad \mathcal{M}_{1} \cap \mathcal{B}_{N}=\mathcal{M}_{2} \cap \mathcal{B}_{N} .
\end{gathered}
$$

Proof: (a) We use the fact that each $D_{\ell}$ is an infinite subset of $\mathbb{N}$.

Write $\mathbf{m}=\chi\left(\ell_{1}\right)+\cdots+\chi\left(\ell_{r}\right)$ where $r=|\mathbf{m}|$. Choose $j_{r} \in D_{\ell_{r}}$ with $j_{r}>N$. For $i=1, \ldots, r-1$ inductively choose $j_{r-i} \in D_{\ell_{r-i}}$ with $j_{r-i}>j_{r-i+1}$. Then $j_{1}, \ldots, j_{r}$ is an expansion for $t \in I P_{+}(k)$ with $\mathbf{r}(t)=\mathbf{m}$.

(b) If $\mathbf{m} \in \mathcal{M}_{1} \backslash \mathcal{M}_{2}$, then by definition $t$ is in $A\left[\mathcal{M}_{1}\right] \backslash A\left[\mathcal{M}_{2}\right]$ when $\mathbf{r}(t)=\mathbf{m}$. If $t \in I P_{+}(k)$ then $t$ is in $A_{+}\left[\mathcal{M}_{1}\right] \backslash A\left[\mathcal{M}_{2}\right]$. Hence, if $\mathcal{M}_{1} \neq \mathcal{M}_{2}$, then $A\left[\mathcal{M}_{1}\right] \neq$ $A\left[\mathcal{M}_{2}\right]$ and $A_{+}\left[\mathcal{M}_{1}\right] \neq A_{+}\left[\mathcal{M}_{2}\right]$. The reverse implications are obvious.

(c) The first implication follows by intersecting with $I P_{+}(k)$.

For each $\mathbf{m} \in \mathcal{B}_{N}$ choose $t=t(\mathbf{m}) \in I P_{+}(k)$ with $\mathbf{r}(t)=\mathbf{m}$ and let $M=$ $\max \left\{t(\mathbf{m}): \mathbf{m} \in \mathcal{B}_{N}\right\}$. Now assume $[ \pm M] \cap A_{+}\left[\mathcal{M}_{1}\right]=[ \pm M] \cap A_{+}\left[\mathcal{M}_{2}\right]$.

If $\mathbf{m} \in \mathcal{M}_{1} \cap \mathcal{B}_{N}$ then $0 \leq t(\mathbf{m}) \leq M$. Hence $t(\mathbf{m}) \in[ \pm M] \cap A\left[\mathcal{M}_{1}\right]=$ $[ \pm M] \cap A\left[\mathcal{M}_{2}\right]$. Hence, $t(\mathbf{m}) \in A\left[\mathcal{M}_{2}\right]$ and so $\mathbf{m} \in \mathcal{M}_{2}$. That is, $\mathcal{M}_{1} \cap \mathcal{B}_{N} \subset$ $\mathcal{M}_{2} \cap \mathcal{B}_{N}$. Symmetrically for the reverse inclusion.

\subsection{Subshifts.}

On $\{0,1\}^{\mathbb{Z}}$ define the ultrametric $d$ by 


$$
d(x, y)=\inf \left\{2^{-N}: N \in \mathbb{Z}_{+} \text {and } x_{t}=y_{t} \text { for all }|t|<N\right\} .
$$

We denote by $S$ the shift homeomorphism on $\{0,1\}^{\mathbb{Z}}$. That is, $S(x)_{t}=x_{t+1}$. Hence, for any $k \in \mathbb{Z} S^{k}(x)_{t}=x_{t+k}$.

For $A \subset \mathbb{Z}$ we defined $\chi(A) \in\{0,1\}^{\mathbb{Z}}$ by $\chi(A)_{t}=1$ iff $t \in A$, so that $\chi(A)$ is the characteristic function of $A$. Thus, $\chi(\emptyset)=e$ with $e_{t}=0$ for all $t$. If $z \in\{0,1\}^{\mathbb{Z}}$ then $z=\chi(A)$ for $A=\left\{t \in \mathbb{Z}: z_{t}=1\right\}$.

Definition 3.14. For $A \subset \mathbb{Z}$, let $X(A)$ be the orbit closure of $\chi(A)$, i.e. the smallest closed invariant subspace of $\{0,1\}^{\mathbb{Z}}$ which contains $\chi(A)$. For $\mathcal{A}$ a nonempty collection of subsets of $\mathbb{Z}$, let $X(\mathcal{A})$ denote the smallest closed invariant subspace which contains $\chi(A)$ for all $A \in \mathcal{A}$. In particular, for $A \subset \mathbb{Z}$ with power set $\mathcal{P} A$, the set $X(\mathcal{P} A)$ is the smallest closed invariant subset of $\{0,1\}^{\mathbb{Z}}$ which contains $\chi\left(A_{1}\right)$ for every $A_{1} \subset A$.

We will say that $x=\chi(A)$ dominates $z=\chi\left(A_{1}\right)$ when $A \supset A_{1}$. Clearly, the set of pairs $\{(x, z)\}$ such that $x$ dominates $z$ is closed.

For $x=\chi(A)$ we will say that $x$ has positive or zero upper Banach density when the subset $A$ has positive or zero upper Banach density, as defined in (3.14) above. The point $x$ has positive upper Banach density iff there exists an invariant probability measure $\mu$ on its orbit closure such that the cylinder set $C_{0}=\left\{z \in\{0,1\}^{\mathbb{Z}}: z_{0}=1\right\}$ has positive $\mu$ measure, see [15, Lemma 3.17]. Observe that the union of the translates $S^{k}\left(C_{0}\right)$ is the complement of $\{e\}$. So for an invariant probability measure $\mu$ the set $C_{0}$ has positive measure iff $\mu$ is not the point measure $\delta_{e}$ where $e$ is the fixed point $\chi(\emptyset)$. Thus, for example, if $X(A)$ contains a minimal subset other than the fixed point $e$ then $A$ has positive upper Banach density.

Theorem 3.15. If $A$ has Banach density zero then every point $x \in X(\mathcal{P} A)$ has Banach density zero. The dynamical system $(X(\mathcal{P} A), S)$ is uniquely ergodic with $\delta_{e}$, the point mass at $e$, as the unique invariant probability measure and so $\{e\}$ is the unique minimal subset of $X(\mathcal{P} A)$. In particular, $e$ is the only periodic point in $X(\mathcal{P} A)$. Furthermore, $(X(\mathcal{P} A), S)$ has entropy zero.

Proof: First observe that any block of a point $x \in X(A)$ is also a block in $\chi(A)$. Thus every such $x$ has Banach density zero. Moreover, every point $y \in X(\mathcal{P} A)$ is dominated by a point $x \in X(A)$ and therefore has also Banach density zero. Since, by Birkhoff ergodic theorem, every ergodic measure admits a generic point we can apply Furstenberg characterization [15, Lemma 3.17 to deduce that the only ergodic measure on $X(\mathcal{P} A)$ is $\delta_{e}$. The ergodic decomposition theorem implies that indeed $\delta_{e}$ is the only invariant probability 
measure on $X(\mathcal{P} A)$. The topological entropy of $X(\mathcal{P} A)$ is zero by the Variational Principle since the entropy of the unique invariant measure is zero (see e.g.[18]). Finally, as any minimal subset carries an invariant probability measure and distinct minimal subsets are disjoint, it follows that $\{e\}$ is the only minimal subset of $X(\mathcal{P} A)$.

Definition 3.16. For a label $\mathcal{M}$ let $x[\mathcal{M}]=\chi(A[\mathcal{M}]), x_{+}[\mathcal{M}]=\chi\left(A_{+}[\mathcal{M}]\right)$. Let $X(\mathcal{M})$ and $X_{+}(\mathcal{M})$ denote the orbit closures $X(A[\mathcal{M}])$ and $X\left(A_{+}[\mathcal{M}]\right)$, respectively. For $\Phi \subset \mathcal{L} \mathcal{A B}$, let $X(\Phi)$ and $X_{+}(\Phi)$ denote $X(\{A[\mathcal{M}]: \mathcal{M} \in \Phi\})$ and $X\left(\left\{A_{+}[\mathcal{M}]: \mathcal{M} \in \Phi\right\}\right)$, respectively.

For example, $x_{+}[\emptyset]=x[\emptyset]=e$ and $x_{+}[0]_{t}=x[0]_{t}=1$ for $t=0$ and $=0$ otherwise. In general, the points $x[\mathcal{M}]$ are symmetric in that $x[\mathcal{M}]_{-t}=x[\mathcal{N}]_{t}$. On the other hand, $x_{+}[\mathcal{M}]_{t}=0$ if $t<0$. Hence, $x[\mathcal{M}] \neq x_{+}[\mathcal{M}]$ if $\mathcal{M}$ is a positive label.

Corollary 3.17. For the subshifts $(X(\mathcal{P} I P(k)), S),(X(\mathcal{L A B}), S)$ and $\left(X_{+}(\mathcal{L} \mathcal{A B}), S\right)$ the point measure $\delta_{e}$ is the unique invariant probability measure and so $\{e\}$ is the unique minimal subset. In particular, the systems are minCT and have entropy zero.

Proof: By Theorem 3.7 IP $(k)$ has Banach density zero. So by Theorem $3.15 \delta_{e}$ is the unique invariant probability measure for $(X(\mathcal{P} I P(k)), S)$ and so the same is true for the subsystems $(X(\mathcal{L A B}), S)$ and

$\left(X_{+}(\mathcal{L} \mathcal{A B}), S\right)$

Theorem 3.18. The maps $x[\cdot], x_{+}[\cdot]$ defined by $\mathcal{M} \mapsto x[\mathcal{M}]$ and $\mathcal{M} \mapsto x_{+}[\mathcal{M}]$ are homeomorphisms from $\mathcal{L} \mathcal{A B}$ onto their images in $\{0,1\}^{\mathbb{Z}}$.

Proof: Let $\mathcal{M}_{1}, \mathcal{M}_{2}$ be labels with $\mathcal{M}_{1} \cap \mathcal{B}_{N}=\mathcal{M}_{2} \cap \mathcal{B}_{N}$ for some $N>1$. By Lemma 3.13 (b) $\mathcal{M}_{1} \cap \mathcal{B}_{N}=\mathcal{M}_{2} \cap \mathcal{B}_{N}$ iff $A\left[\mathcal{M}_{1} \cap \mathcal{B}_{N}\right]=A\left[\mathcal{M}_{2} \cap \mathcal{B}_{N}\right]$. By Proposition 3.12 this implies $[ \pm N] \cap A\left[\mathcal{M}_{1}\right]=[ \pm N] \cap A\left[\mathcal{M}_{2}\right]$ and so, by intersecting with $I P_{+}(k)$, that $[ \pm N] \cap A_{+}\left[\mathcal{M}_{1}\right]=[ \pm N] \cap A_{+}\left[\mathcal{M}_{2}\right]$ these are equivalent to $x\left[\mathcal{M}_{1}\right]_{t}=x\left[\mathcal{M}_{2}\right]_{t}$ and $x_{+}\left[\mathcal{M}_{1}\right]_{t}=x_{+}\left[\mathcal{M}_{2}\right]_{t}$ for all $t \in$ $[ \pm N]$. Since $d(\emptyset, 0)=1=d(x[\emptyset], x[0])$, it follows that $x[\cdot]$ has Lipschitz constant 1.

The map $\mathcal{M} \mapsto A_{+}[\mathcal{M}]$ is injective by Lemma 3.13 (b). Clearly, $A \mapsto \chi(A)$ is injective. So compactness implies that $x[\cdot]$ and $x_{+}[\cdot]$ are homeomorphisms onto their respective images. 
Corollary 3.19. If $\left\{\mathcal{M}^{i}\right\}$ is a net of labels then $\left\{\mathcal{N}^{i}\right\}$ is convergent iff $\left\{x\left[\mathcal{M}^{i}\right]\right\}$ is convergent and iff $\left\{x_{+}\left[\mathcal{M}^{i}\right]\right\}$ is convergent.

The following is the crucial result which relates the $F I N(\mathbb{N})$ dynamics on $\mathcal{L} \mathcal{A B}$ with the shift dynamics on the $\{0,1\}^{\mathbb{Z}}$.

Lemma 3.20. (Coherence Lemma) Let $\left\{t^{i}\right\}$ be a net in $I P(k)$ with $r^{i}$ the length of $t^{i}$. If $\left\{\left|j_{r^{i}}\left(t^{i}\right)\right|\right\} \rightarrow \infty$ then

$$
\operatorname{Lim}_{i \rightarrow \infty} \sup _{\mathcal{M} \in \mathcal{L} \mathcal{A B B}} d\left(S^{t^{i}}(x[\mathcal{M}]), x\left[\mathcal{M}-\mathbf{r}\left(t^{i}\right)\right]\right)=0 .
$$

Furthermore, if $t^{i} \in I P_{+}(k)$ for $i \in I$ then

$$
\operatorname{Lim}_{i \rightarrow \infty} \sup _{\mathcal{M} \in \mathcal{L A B}} d\left(S^{t^{i}}\left(x_{+}[\mathcal{M}]\right), x_{+}\left[\mathcal{M}-\mathbf{r}\left(t^{i}\right)\right]\right)=0 .
$$

That is, the pair of nets $\left(\left\{S^{t^{i}}(x[\mathcal{M}])\right\},\left\{x\left[\mathcal{M}-\mathbf{r}\left(t^{i}\right)\right]\right\}\right)$ is uniformly asymptotic in $X(\mathcal{L A B})$ and if $t^{i} \in I P_{+}(k)$ for all $i \in I$ then the pair

$$
\left(\left\{S^{t^{i}}\left(x_{+}[\mathcal{N}]\right)\right\},\left\{x_{+}\left[\mathcal{M}-\mathbf{r}\left(t^{i}\right)\right]\right\}\right)
$$

is uniformly asymptotic in $X_{+}(\mathcal{L} \mathcal{A B})$.

Proof: For any positive integer $N$, there exists $i_{N}$ in the directed set $I$ which indexes the net, so that $i>i_{N}$ implies $\left|j_{r^{i}}\left(t^{i}\right)\right|>N$. Then Proposition 3.10 implies for any label $\mathcal{M}\left[t^{i} \pm N\right] \cap A[\mathcal{M}]=t^{i}+\left([ \pm N] \cap A\left[\mathcal{N}-\mathbf{r}\left(t^{i}\right)\right]\right)$.

This says that for all $t$ with $|t| \leq N, x\left[\mathcal{M}-\mathbf{r}\left(t^{i}\right)\right]_{t}=x[\mathcal{M}]_{t^{i}+t}$. Thus, if $i>i_{N}$, then $x\left[\mathcal{M}-\mathbf{r}\left(t^{i}\right)\right]_{t}=\left(S^{t^{i}}(x[\mathcal{M}])\right)_{t}$ for all $t$ with $|t| \leq N$. So (3.20) follows from the definition (3.19) of the metric on $\{0,1\}^{\mathbb{Z}}$.

The proof for $x_{+}$is the same.

Lemma 3.21. For a label $\mathcal{M}$ assume that $I$ is a directed set and $\left\{t^{i}: i \in I\right\}$ is a net in $A[\mathcal{M}] \backslash\{0\}$ with $r^{i}$ the length of $t^{i}$. Assume that $\left\{\left|j_{r^{i}}\left(t^{i}\right)\right|\right\}$ is bounded.

(a) There exists an integer $j^{*} \neq 0$ such that $\left\{i \in I: j_{r^{i}}\left(t^{i}\right)=j^{*}\right\}$ is a cofinal subset.

(b) If $\mathcal{M}$ is a label of finite type and $\left|t^{i}\right| \rightarrow \infty$, then there exists $\bar{t} \in A[\mathcal{M}]$ with length $\bar{r}>0$ (and so $\bar{t} \neq 0$ ) and a subnet $\left\{t^{i^{\prime}}\right\}$, defined by restricting to $i^{\prime} \in I^{\prime}$, a cofinal subset of $I$, such that $t^{i^{\prime}}-\tilde{t}^{i^{\prime}}=\bar{t}$ for all $i^{\prime}$, where $\tilde{t}^{i^{\prime}}$ is the $\tilde{r}^{i^{\prime}}=r^{i^{\prime}}-\bar{r}$ truncation of $t^{i^{\prime}}$ and, in addition, $\left|j_{\tilde{r}^{i^{\prime}}}\left(\tilde{t}^{i^{\prime}}\right)=j_{\tilde{r}^{i^{\prime}}}\left(t^{i^{\prime}}\right)\right| \rightarrow \infty$.

Proof: The bounded set $\left\{j_{r^{i}}\left(t^{i}\right)\right\}$ is finite. It follows that there exists $j^{-1} \in \mathbb{Z} \backslash\{0\}$ such that $j_{r^{i}}\left(t^{i}\right)=j^{-1}$ for $i$ in a cofinal subset $S E Q_{-1}$ of I. Let $\bar{t}_{-1}=k\left(j^{-1}\right)$ and let $\tilde{t}_{-1}^{i}$ be the $\tilde{r}_{-1}^{i}=r^{i}-1$ truncation of $t^{i}$ for all $i$ in $S E Q_{-1}$. 
Since $j^{-1} \neq 0, \bar{t}_{-1} \neq 0$. Furthermore, $\mathbf{r}\left(t^{i}\right)=\mathbf{r}\left(\tilde{t}_{-1}^{i}\right)+\chi\left(\ell\left(j^{-1}\right)\right) \in \mathcal{M}$ for all $i \in S E Q_{-1}$.

For (a) we let $j^{*}=j^{-1}$ and $I^{\prime}=S E Q_{-1}$.

Now we assume that $\mathcal{M}$ is of finite type and we continue in order to prove (b).

If $\left\{\left|j_{\tilde{r}_{-1}^{i}}\left(\tilde{t}_{-1}^{i}\right)\right|\right\} \rightarrow \infty$ as $i \rightarrow \infty$ in $S E Q_{-1}$ then let $I^{\prime}=S E Q_{-1}$ use this as the required subnet. Otherwise, it is bounded on some cofinal subset and we can choose $j^{-2} \in \mathbb{Z} \backslash\{0\}$ such that $j_{\tilde{r}_{-1}^{i}}\left(\tilde{t}_{-1}^{i}\right)=j^{-2}$, for $i$ in a cofinal subset $S E Q_{-2}$ of $S E Q_{-1}$. Let $\bar{t}_{-2}=k\left(j^{-2}\right)+k\left(j^{-1}\right)$ and let $\tilde{t}_{-2}^{i}$ be the $\tilde{r}_{-2}^{i}=r^{i}-2$ truncation of $t^{i}$ for all $i$ in $S E Q_{-2}$. The truncation is proper and $\bar{t}_{-2} \neq 0$. Furthermore, $\mathbf{r}\left(t^{i}\right)=\mathbf{r}\left(\tilde{t}_{-2}^{i}\right)+\chi\left(\ell\left(j^{-1}\right)\right)+\chi\left(\ell\left(j^{-2}\right)\right) \in \mathcal{M}$ for all $i \in S E Q_{-2}$.

Because $\mathcal{M}$ is of finite type, the Finite Chain Condition implies that this procedure must halt after finitely many steps. That is, for some $k \geq 1$, $\left\{\left|j_{\tilde{r}_{-k}^{i^{\prime}}}\left(\tilde{t}_{-k}^{i^{\prime}}\right)\right|\right\} \rightarrow \infty$ as $i^{\prime} \rightarrow \infty$ in $S E Q_{-k}$. The subnet is obtained by restricting to $I^{\prime}=S E Q_{-k}$ and $\bar{t}=\bar{t}_{-k}$ with length $\bar{r}=k$.

Since $\tilde{t}^{i^{\prime}}$ is the truncation of $t^{i^{\prime}}$ to $\tilde{r}^{i^{\prime}}$, we have $\left|j_{\tilde{r}^{\prime}}\left(\tilde{t}^{i^{\prime}}\right)=j_{\tilde{r}^{i^{\prime}}}\left(t^{i^{\prime}}\right)\right| \rightarrow \infty$.

Remark 3.22. In (b) if $t^{i} \in A_{+}[\mathcal{M}]$ for all $i$ then $\bar{t} \in A_{+}[\mathcal{M}]$ and the truncations are in $I P_{+}(k)$.

Definition 3.23. For a nonempty subset $A \subset \mathbb{Z}$ define the distance from $t$ to $A$ by $\xi_{A}: \mathbb{Z} \rightarrow \mathbb{Z}_{+}$by

$$
\xi_{A}(t)=\min \{n: t+n \in A \text { or } t-n \in A\} .
$$

Thus, $\xi_{A}(t)=0$ iff $t \in A$.

For $\mathcal{M}$ a nonempty label, we denote $\xi_{A[\mathcal{N}]}$ by $\xi_{\mathcal{M}}$ and $\xi_{A_{+}[\mathcal{M}]}$ by $\xi_{\mathcal{M}}^{+}$. From the symmetry of $A[\mathcal{M} \mathbb{M}]$ we see that $\xi_{\mathcal{M}}(-t)=\xi_{\mathcal{M}}(t)$ for all $t$. On the other hand, $\xi_{\mathcal{M}}^{+}(t)=|t|$ if $t \leq 0$.

Recall that $e \in\{0,1\}^{\mathbb{Z}}$ is the fixed point of $S$ with $e_{t}=0$ for all $t$.

Lemma 3.24. Let $I$ be a directed set, $\left\{t^{i}: i \in I\right\}$ be a net in $\mathbb{Z}$ and $\left\{A^{i}: i \in I\right\}$ be a net of nonempty subsets of $\mathbb{Z}$. The net $\left\{S^{t^{i}}\left(x\left[A^{i}\right]\right): i \in I\right\}$ in $\{0,1\}^{\mathbb{Z}}$ converges to e iff $\xi_{A^{i}}\left(t_{i}\right) \rightarrow \infty$.

Proof: For any net $\left\{z^{i}\right\} \in\{0,1\}^{\mathbb{Z}}, S^{t^{i}}\left(z^{i}\right) \rightarrow e$ iff for all $n \in \mathbb{N}$ eventually $z_{t^{i}+k}^{i}=0$ for all $k$ with $|k|<n$.

For $t \in \mathbb{Z} \backslash\{0\}$, recall that $n_{*}(t)$ is the place of the smallest nonzero entry in the $b$ expansion of $t$ so that when $t \in I P(k), n_{*}(t)=\left|j_{r}(t)\right|$ with $r=r(t)$. 
Theorem 3.25. Let $I$ be a directed set, $\left\{t^{i}: i \in I\right\}$ be a net of nonzero integers and $\left\{\mathcal{M}^{i}: i \in I\right\}$ be a net of labels in $\mathcal{L} \mathcal{A B}$.

If the net $\left\{S^{t^{i}}\left(x\left[\mathcal{M}^{i}\right]\right): i \in I\right\}$ converges to $z \in\{0,1\}^{\mathbb{Z}}$ with $z \neq e$, then the following are equivalent.

(i) $z_{0}=1$ and $z_{t}=z_{-t}$ for all $t \in \mathbb{Z}$.

(ii) $z=x[\mathcal{N}]$ for some label $\mathcal{N}$.

(iii) $n_{*}\left(t^{i}\right) \rightarrow \infty$.

(iv) There exists $i_{0} \in I$ so that on the subnet obtained by restricting to $i>i_{0}, t^{i} \in A\left[\mathcal{M}^{i}\right]$ and $\left|j_{r_{i}}\left(t^{i}\right)\right| \rightarrow \infty$ with $r_{i}$ the length of $t^{i}$.

If the net $\left\{S^{t^{i}}\left(x_{+}\left[\mathcal{M}^{i}\right]\right): i \in I\right\}$ converges to $w \in\{0,1\}^{\mathbb{Z}}$ with $w \neq e$, then the following are equivalent.

(i') $w_{0}=1$ and $w_{t}=0$ for all $t<0 \in \mathbb{Z}$.

(ii') $w=x_{+}[\mathcal{N}]$ for some label $\mathcal{N}$.

(iii') $n_{*}\left(t^{i}\right) \rightarrow \infty$.

(iv') There exists $i_{0} \in I$ so that on the subnet obtained by restricting to $i>i_{0}, t^{i} \in A_{+}\left[\mathcal{M}^{i}\right]$ and $\left|j_{r_{i}}\left(t^{i}\right)\right| \rightarrow \infty$ with $r_{i}$ the length of $t^{i}$.

When either of these sets of conditions hold the label $\mathcal{N}$ of (ii) or (ii') is $L I M_{i>i_{0}}\left\{\mathcal{M}^{i}-\mathbf{r}\left(t^{i}\right)\right\}$. In particular, if $\mathcal{M}=\mathcal{M}^{i}$ for all $i$ then $\mathcal{N} \in \Theta^{\prime}(\mathcal{M})$.

Proof: Since $z$ (or $w$ ) is not $e$, the labels $\mathcal{M}^{i}$ are eventually nonempty.

(iii) $\Rightarrow$ (iv): If for some subnet $\left\{t^{i^{\prime}}\right\}$, we have $\xi_{\mathcal{M}^{i}}\left(t^{i^{\prime}}\right) \rightarrow \infty$ then by Lemma 3.24 the subnet $\left\{S^{t^{i^{\prime}}}\left(x\left[\mathcal{M}^{i}\right]\right)\right\}$ converges to $e$. Since $z \neq e$ this does not happen. By the Smith Lemma A.1 it follows that for some $N \in \mathbb{N}$ the set $\{i \in I$ : $\left.\xi_{\mathcal{M}^{i}}\left(t^{i}\right)>N\right\}$ is not cofinal and so for some $i_{0} \in I, \xi_{\mathcal{M}^{i}}\left(t^{i}\right) \leq N$ for all $i \geq i_{0}$. It follows that there is a nonempty subset $K$ of the finite interval $[-N, N]$ so that for each $k \in K$,

$$
I_{k}=\left\{i \in I: t^{i}+k \in A\left[\mathcal{M}^{i}\right] \text { and } t^{i}+j \notin A\left[\mathcal{M}^{i}\right] \text { when }|j|<|k|\right\}
$$

is cofinal. Thus, $I_{k} \cup I_{-k}=\left\{i: \xi_{\mathcal{M}^{i}}\left(t^{i}\right)=|k|\right\}$. We will show that $K=\{0\}$.

Let $k \in K$. By assumption (iii) eventually $n_{*}\left(t^{i}\right)>n^{*}(k)$. This implies that the places where $t^{i}$ has a nonzero entry in its $b$ expansion are beyond the places where $k$ has a nonzero entry. On the cofinal set with $i \in I_{k}$ and $n_{*}\left(t^{i}\right)>n^{*}(k)$, we have that $t^{i}+k \in I P(k)$ and that $t^{i}$ is a truncation of $t^{i}+k$. Hence $\mathbf{r}\left(t^{i}\right) \leq \mathbf{r}\left(t^{i}+k\right) \in \mathcal{M}^{i}$ and so $t^{i} \in A\left[\mathcal{M}^{i}\right]$. Thus, $|k|=\xi_{\mathcal{M}}\left(t^{i}\right)=0$. It thus follows that $\left\{i: \xi_{\mathcal{M}^{i}}\left(t^{i}\right) \neq 0\right\}$ is not cofinal.

That is, eventually $t^{i} \in A\left[\mathcal{M}^{i}\right] \subset I P(k)$. For $t^{i} \in I P(k), n_{*}\left(t^{i}\right)=\left|j_{r^{i}}\left(t^{i}\right)\right|$ and so (iv) follows.

(iii') $\Rightarrow$ (iv'): Use the same proof as above replacing $\xi_{\mathcal{M}^{i}}$ by $\xi_{\mathcal{M}^{i}}^{+}$.

(iv) $\Rightarrow$ (iii), (iv') $\Rightarrow$ (iii'): Obvious.

(iv) $\Rightarrow\left(\right.$ ii), (iv') $\Rightarrow\left(\right.$ ii' $\left.^{\prime}\right)$ with $\mathcal{N}=\operatorname{Lim}\left\{\mathcal{M}^{i}-\mathbf{r}\left(t^{i}\right)\right\}$ : Since $\left\{S^{t^{i}}\left(x_{+}\left[\mathcal{M}^{i}\right]\right)\right\}$ is convergent the Coherence Lemma 3.20 implies that $\left\{x\left[\mathcal{N}^{i}-\mathbf{r}\left(t^{i}\right)\right]\right\}$ is 
convergent. By Corollary 3.19 it follows that $\left\{\mathcal{M}^{i}-\mathbf{r}\left(t^{i}\right)\right\}$ is convergent in $\mathcal{L A B}$. If $\mathcal{N}$ is its limit then Theorem 3.18 implies that $\left\{x\left[\mathcal{M}^{i}-\mathbf{r}\left(t^{i}\right)\right]\right\}$ and so $\left\{S^{t^{i}}\left(x_{+}\left[\mathcal{M}^{i}\right]\right)\right\}$ both converge to $x[\mathcal{N}]$.

(ii) $\Rightarrow$ (i), (ii') $\Rightarrow$ (i'): Obvious.

(i) $\Rightarrow$ (iv): Since $z_{0}=1$ it follows that eventually $S^{t^{i}}\left(x\left[\mathcal{N}^{i}\right]\right)_{0}=x\left[\mathcal{M}^{i}\right]_{t^{i}}=1$ and so eventually $t^{i} \in A\left[\mathcal{N}^{i}\right]$. Assume this is true for all $i \geq i_{0}$.

We have to show that $\left|j_{r^{i}}\left(t^{i}\right)\right| \rightarrow \infty$. If this is not true then on some cofinal set $\left|j_{r^{i}}\left(t^{i}\right)\right|$ remains bounded. From Lemma 3.20 there is a nonzero $j^{*}$ and a cofinal set $I^{\prime}$ such that $j_{r^{i}}\left(t^{i}\right)=j^{*}$ for all $i \in I^{\prime}$. Let $\tilde{t}^{i}=t^{i}-k\left(j^{*}\right)$ so that $\tilde{t}^{i}$ is a truncation of $t^{i}$ and so $\tilde{t}^{i} \in A\left[\mathcal{M}^{i}\right] \subset I P(k)$. On the other hand $t^{i}+k\left(j^{*}\right) \notin I P(k)$ because it has a 2 or a -2 at the $\left|j^{*}\right|$ place in its expansion. Thus, for $i \in I^{\prime}$ we have $S^{t^{i}}\left(x\left[\mathcal{M}^{i}\right]\right)_{-k\left(j^{*}\right)}=1$, but $S^{t^{i}}\left(x\left[\mathcal{M}^{i}\right]\right)_{k\left(j^{*}\right)}=0$. Since $S^{t^{i}}\left(x\left[\mathcal{M}^{i}\right]\right) \rightarrow z$ we have $z_{-k\left(j^{*}\right)}=1$ and $z_{k\left(j^{*}\right)}=0$ which contradicts the symmetry assumption on $z$.

(i') $\Rightarrow$ (iv'): Begin as above. Now $t^{i} \in A_{+}\left[\mathcal{M}^{i}\right]$ and so $j^{*}>0$. As before we have $S^{t^{i}}\left(x\left[\mathcal{M}^{i}\right]\right)_{-k\left(j^{*}\right)}=1$ and so in the limit $w_{-k\left(j^{*}\right)}=1$. This contradicts the assumption that $w_{t}=0$ for all $t<0$.

Corollary 3.26. For a label $\mathcal{M}$ the following are equivalent.

(i) $\mathcal{M}$ is recurrent for the $F I N(\mathbb{N})$ action on $\mathcal{L} \mathcal{A B}$.

(ii) $x[\mathcal{M}]$ is a recurrent point for the shift homeomorphism on $\{0,1\}^{\mathbb{Z}}$.

(iii) $x_{+}[\mathcal{M}]$ is a recurrent point for the shift homeomorphism on $\{0,1\}^{\mathbb{Z}}$.

Proof: We may assume $\mathcal{M} \neq \emptyset$ since all three properties hold when $\mathcal{M}=\emptyset$.

(i) $\Rightarrow$ (ii), (iii): Recall that $\mathcal{M}$ is recurrent when $\mathcal{M}=\operatorname{LIM}\left\{\mathcal{M}-\mathbf{r}^{i}\right\}$ for some sequence $\mathbf{r}^{i}>0$ in $F I N(\mathbb{N})$. By Lemma 3.13 (a) and induction, we can choose a sequence $\left\{t^{i} \in I P_{+}(k)\right\}$ with $\mathbf{r}\left(t^{i}\right)=\mathbf{r}^{i}$ and with $j_{r}\left(t^{i}\right) \rightarrow \infty$. By the Coherence Lemma $3.20\left\{S^{t^{i}}(x[\mathcal{M}])\right\}$ is asymptotic to $\left\{x\left[\mathcal{M}-\mathbf{r}^{i}\right]\right\}$ which converges to $x[\mathcal{M}]$ by Theorem 3.18. Similarly, $\left\{S^{t^{i}}\left(x_{+}[\mathcal{M}]\right)\right\}$ is asymptotic to $\left\{x_{+}\left[\mathcal{M}-\mathbf{r}^{i}\right]\right\}$ which converges to $x_{+}[\mathcal{N}]$.

(ii) $\Rightarrow(\mathrm{i}): x[\mathcal{M}]$ is recurrent when there is a sequence $\left\{t^{i} \in \mathbb{Z} \backslash\{0\}\right\}$ such that $\left\{S^{t^{i}}(x[\mathcal{M}])\right\}$ converges to $x[\mathcal{M}]$. By Theorem 3.25 (ii) $\Rightarrow$ (iv), we have $t^{i} \in$ $A[\mathcal{M}]$ with $j_{r}\left(t^{i}\right) \rightarrow \infty$. By the Coherence Lemma 3.20 again, $\left\{S^{t^{i}}(x[\mathcal{M}])\right\}$ is asymptotic to $\left\{x\left[\mathcal{M}-\mathbf{r}\left(t^{i}\right)\right]\right\}$ and so the latter converges to $x[\mathcal{M}]$. By Theorem 3.18, $\left\{\mathcal{M}-\mathbf{r}\left(t^{i}\right)\right\}$ converges to $\mathcal{M}$ and so $\mathcal{M}$ is recurrent.

(iii) $\Rightarrow(\mathrm{i})$ : Since $x_{+}[\mathcal{M}] \neq e, x_{+}[\mathcal{M}]$ is recurrent when there is a sequence $\left\{t^{i} \in \mathbb{N}\right\}$ such that $\left\{S^{t^{i}}\left(x_{+}[\mathcal{M}]\right)\right\}$ converges to $x_{+}[\mathcal{M}]$. Apply Theorem 3.25 (ii') $\Rightarrow$ (iv') and the Coherence Lemma as before to show that $\mathcal{M}$ is recurrent. 
Now we interpret these results in terms of the Ellis action of $\beta \mathbb{Z}$ on $\{0,1\}^{\mathbb{Z}}$, see Appendix C.

Since $n_{*}(0)=\infty, n_{*}$ is a function from $\mathbb{Z}$ to the compact space $\mathbb{Z}_{+\infty}$. For $A$ a nonempty subset $\mathbb{Z}$ we can similarly regard $\xi_{A}$ as a function from $\mathbb{Z}$ to $\mathbb{Z}_{+\infty}$. So we obtain the continuous extensions

$$
\beta n_{*}: \beta \mathbb{Z} \rightarrow \mathbb{Z}_{+\infty}, \quad \beta \xi_{A}: \beta \mathbb{Z} \rightarrow \mathbb{Z}_{+\infty}
$$

The function $m_{b}: \mathbb{Z} \rightarrow \mathbb{Z}$ defined by $t \mapsto b t$ is an injective group homomorphism and so extends to

$$
\beta m_{b}: \beta \mathbb{Z} \rightarrow \beta \mathbb{Z}
$$

a continuous, injective monoid homomorphism which is injective by Lemma C.1. Hence, its image is a closed submonoid. Define

$$
\beta_{b} \mathbb{Z}=\bigcap_{k=1}^{\infty}\left(\beta m_{b}\right)^{k}(\beta \mathbb{Z}) .
$$

Proposition 3.27. The subset $\beta_{b} \mathbb{Z}$ is an uncountable, closed submonoid of $\beta \mathbb{Z}$ on which $\beta m_{b}$ restricts to an isomorphism. Furthermore,

$$
\beta_{b} \mathbb{Z}=\left(\beta n_{*}\right)^{-1}(\infty) .
$$

Proof: As above $\beta b_{m}$ is injective.

If $p \in \beta_{b} \mathbb{Z} \subset \beta m_{b}(\beta \mathbb{Z})$ there is a unique $q \in \beta \mathbb{Z}$ such that $p=\beta m_{b}(q)$. For $k \geq 2, p \in\left(\beta m_{b}\right)^{k}(\beta \mathbb{Z})$ implies $q \in\left(\beta m_{b}\right)^{k-1}(\beta \mathbb{Z})$ since $\beta m_{b}$ is injective. Hence, $q \in \bigcap_{k=1}^{\infty}\left(\beta m_{b}\right)^{k}(\beta \mathbb{Z})=\beta_{b} \mathbb{Z}$. That is, $\beta m_{b}$ is bijective on $\beta_{b} \mathbb{Z}$.

As it is the intersection of closed submonoids, $\beta_{b} \mathbb{Z}$ is a closed submonoid. Define the injection $j: \mathbb{N} \rightarrow \mathbb{Z}$ by $j(n)=b^{n}$. By Lemma C.1 the restriction of $\beta j$ to $\beta^{*} \mathbb{N}$ is an injection of an uncountable set into $\beta_{b} \mathbb{Z}$. Hence, $\beta_{b} \mathbb{Z}$ is uncountable.

From (3.2) it follows that $m_{b}^{k}(\mathbb{Z})=\left\{n_{*}>k\right\}$. Taking the closure in $\beta \mathbb{Z}$ we obtain (3.26).

Proposition 3.28. Let $\mathcal{M}$ be a nonempty label and $p \in \beta \mathbb{Z}$.

(a) The subsets $\left(\beta \xi_{\mathcal{M}}\right)^{-1}(\infty)$ and $\left(\beta \xi_{\mathcal{M}}^{+}\right)^{-1}(\infty)$ are closed, uncountable ideals of $\beta^{*} \mathbb{Z}$. Furthermore,

$$
\begin{aligned}
\left(\beta \xi_{\mathcal{M}}\right)^{-1}(\infty) & =\{p \in \beta \mathbb{Z}: p x[\mathcal{M}]=e\} \\
\left(\beta \xi_{\mathcal{M}}^{+}\right)^{-1}(\infty) & =\left\{p \in \beta \mathbb{Z}: p x_{+}[\mathcal{M}]=e\right\}
\end{aligned}
$$

(b) If $p \in \beta_{b} \mathbb{Z}$ then $p x[\mathcal{M}]=x[\mathcal{N}]$ for some label $\mathcal{N}$. Conversely, if $p x[\mathcal{M}]=$ $x[\mathcal{N}]$ for some nonempty label $\mathcal{N}$ then $p \in \beta_{b} \mathbb{Z}$. 
Proof: Let $\left\{t^{i}\right\}$ be a net in $\mathbb{Z}$ which converges to $p$. Then $\left\{S^{t^{i}}(x[\mathcal{M}])\right\}$ is a net in $\{0,1\}^{\mathbb{Z}}$ which converges to $p x[\mathcal{M}]$ and $\left\{S^{t^{i}}\left(x_{+}[\mathcal{M}]\right)\right\}$ converges to $p x_{+}[\mathcal{M}]$. Furthermore, $\left\{\xi_{\mathcal{M}}\left(t^{i}\right)\right\} \rightarrow \beta \xi_{\mathcal{M}}(p),\left\{\xi_{\mathcal{M}}^{+}\left(t^{i}\right)\right\} \rightarrow \beta \xi_{\mathcal{M}}^{+}(p)$ and $\left\{n_{*}\left(t^{i}\right)\right\} \rightarrow \beta n_{*}(p)$.

(a): From Lemma 3.24 it follows that $p x[\mathcal{M}]=e$ iff $\beta n_{*}(p)=\infty$ and similarly for $x^{+}[\mathcal{M}]$. If $q \in \beta \mathbb{Z}$ and $p x[\mathcal{M}]=e$ then $q p x[\mathcal{M}]=e$. Hence, $\{p \in \beta \mathbb{Z}: p x[\mathcal{M}]=e\}$ is an ideal. Since $A[\mathcal{M}]$ has Banach density zero, we can choose $j: \mathbb{N} \rightarrow \mathbb{N}$ increasing and so that $\xi_{\mathcal{M}}(j(n)+i)=0$ for all $i$ with $|i| \leq n$. It is clear that $\beta j\left(\beta^{*} \mathbb{N}\right)$ is an uncountable subset of $\{p \in \beta \mathbb{Z}: p x[\mathcal{M}]=e\}$. Finally, since $\mathcal{M}$ is nonempty, $\xi_{\mathcal{M}}(t)$ is finite for all $t \in \mathbb{Z}$ and so $\{p \in \beta \mathbb{Z}$ : $p x[\mathcal{M}]=e\} \subset \beta^{*} \mathbb{Z}$. Use similar arguments for $x^{+}[\mathcal{M}]$.

(b): If $\mathcal{N}$ is nonempty, it follows from Theorem 3.25 that if $p x[\mathcal{N}]=x[\mathcal{N}]$ then $\beta n_{*}(p)=\infty$ and, conversely, if $\beta n_{*}(p)=\infty$ and $p x[\mathcal{M}] \neq e$ then $p x[\mathcal{M}]=$ $x[\mathcal{N}]$ for some nonempty label $\mathcal{N}$. Finally, if $p x[\mathcal{M}]=e$ then $p x[\mathcal{M}]=x[\mathcal{N}]$ with $\mathcal{N}=\emptyset$. Again, use similar arguments for $x^{+}[\mathcal{M}]$.

From the $\beta F I N(\mathbb{N})$ Ellis action on $\mathcal{L} \mathcal{A B}$ we defined the enveloping semigroup $\mathcal{E}(\mathcal{L} \mathcal{A B}) \subset \mathcal{L} \mathcal{A} \mathcal{B}^{\mathcal{L A B}}$ with the unique minimal idempotent $U$ given by $U(\mathcal{M})=\emptyset$ for $\mathcal{M} \neq F I N(\mathbb{N})$, see Proposition 2.30. On the other hand, $P(F I N(\mathbb{N}))=F I N(\mathbb{N})$ for all $P \in \mathcal{E}(\mathcal{L A B})$, including $U$. We define $\hat{U} \in$ $\mathcal{L} \mathcal{A B} \mathcal{B}^{\mathcal{L} \mathcal{B}}$ by $\hat{U}(\mathcal{M})=\emptyset$ for all $\mathcal{M}$ including $F I N(\mathbb{N})$ and let $\hat{\mathcal{E}}(\mathcal{L} \mathcal{A B})=$ $\mathcal{E}(\mathcal{L} \mathcal{A B}) \cup\{\hat{U}\}$. This is an Ellis semigroup with minimal idempotent $\hat{U}$.

Define:

$$
\begin{aligned}
D, D_{+}: \beta_{b} \mathbb{Z} \rightarrow \hat{\mathcal{E}}(\mathcal{L} \mathcal{A B}) \quad \text { by } \\
D(p)(\mathcal{M})=\mathcal{N} \quad \Longleftrightarrow p x[\mathcal{M}]=x[\mathcal{N}] \\
D_{+}(p)(\mathcal{M})=\mathcal{N} \quad \Longleftrightarrow p x_{+}[\mathcal{M}]=x_{+}[\mathcal{N}]
\end{aligned}
$$

That is,

$$
p x[\mathcal{M}]=x[D(p)(\mathcal{M})] \text { and } p x_{+}[\mathcal{M}]=x_{+}\left[D_{+}(p)(\mathcal{M})\right] .
$$

Theorem 3.29. The maps $D, D_{+}: \beta_{b} \mathbb{Z} \rightarrow \hat{\mathcal{E}}(\mathcal{L} \mathcal{A B})$ are continuous, surjective monoid homomorphisms.

Let $\left\{t^{i}: i \in I\right\}$ be a net in $\mathbb{Z}$ converging to $p$ in $\beta_{b} \mathbb{Z}$.

(a) If $t^{i} \in I P(k)$ for all $i$ then $D(p)=\operatorname{LIM}\left\{P_{\mathbf{r}\left(t^{i}\right)}\right\}$ in $\mathcal{E}(\mathcal{L} \mathcal{A B})$.

(b) If $t^{i} \in I P_{+}(k)$ for all $i$ then $D_{+}(p)=L I M\left\{P_{\mathbf{r}\left(t^{i}\right)}\right\}$ in $\mathcal{E}(\mathcal{L} \mathcal{A B})$.

(c) If $\left\{i: t^{i} \notin I P(k)\right\}$ is cofinal, then $D(p)=\hat{U}$.

(d) If $\left\{i: t^{i} \notin I P_{+}(k)\right\}$ is cofinal, then $D_{+}(p)=\hat{U}$.

Proof: Since the map $\mathcal{M} \mapsto x[\mathcal{M}]$ is injective, Proposition 3.28 the maps $D$ and $D_{+}$are well-defined by (3.29). For $q, p \in \beta_{b} \mathbb{Z}$ and an arbitrary label $\mathcal{M}$

$$
x[D(q p)(\mathcal{M})]=q p x[\mathcal{M}])=q x[D(p)(\mathcal{M})]=x[D(q)(D(p)(\mathcal{M}))] .
$$


Hence, $D$ is a homomorphism.

If $\left\{p^{i}\right\}$ is a net in $\beta_{b} \mathbb{Z}$ converging to $p$, then

$$
x[D(p)(\mathcal{M})]=p x[\mathcal{M}]=L I M p^{i} x[\mathcal{M}]=L I M x\left[D\left(p^{i}\right)(\mathcal{M})\right] .
$$

So by Theorem $3.18 D(p)(\mathcal{M})=L I M D\left(p^{i}\right)(\mathcal{M})$. Since $\mathcal{M}$ is arbitrary, $D$ is continuous.

The limit results of (a) follow from Theorem 3.25 since $n_{*}\left(t^{i}\right) \rightarrow \infty$. Furthermore, if the limit $p x[\mathcal{M}]$ is not $e$ for some $\mathcal{M}$ then by Theorem 3.25 eventually $t^{i}$ is in $A[\mathcal{M}] \subset I P(k)$. So (c) follows as well. In particular, choosing a sequence $t^{n} \in \mathbb{N} \backslash I P_{+}(k)$ with $n_{*}\left(t^{n}\right) \rightarrow \infty$ we see that the for any $p$ in the set of limit points of the sequence, $p \in \beta_{b} \mathbb{Z}$ and $D(p)=\hat{U}$.

Note that $0 \in \beta_{b} \mathbb{Z}$ and $D(0)=i d_{\mathcal{L A B}}=P_{\mathbf{0}}$.

If $P \neq i d_{\mathcal{L A B}} \in \mathcal{E}(\mathcal{L} \mathcal{A B})$ then we can choose a net $\left\{\mathbf{r}^{i}>\mathbf{0}: i \in I\right\}$ such that $\left\{P_{\mathbf{r}^{i}}\right\} \rightarrow P$. On the product directed set $I \times \mathbb{N}$ apply Lemma 3.13 to choose $t^{(i, n)}$ so that $\mathbf{r}\left(t^{(i, n)}\right)=\mathbf{r}^{i}$ and $j_{r}\left(t^{(i, n)}\right)>n$. Since $\left\{j_{r}\left(t^{(i, n)}\right)\right\} \rightarrow \infty$, $S^{t^{(i, n)}}(x[\mathcal{M}])$ is asymptotic to $\left\{x\left[\mathcal{M}-\mathbf{r}\left(t^{(i, n)}\right)\right]=x\left[\mathcal{M}-\mathbf{r}^{i}\right]\right\}$ which has limit $x[P(\mathcal{M})]$. Also $\left\{n_{*}\left(t^{(i, n)}\right)=j_{r}\left(t^{(i, n)}\right)\right\} \rightarrow \infty$ implies that any limit point $p$ of the net $\left\{t^{(i, n)}\right\}$ in $\beta \mathbb{Z}$ is in fact in $\beta_{b} \mathbb{Z}$, and $D(p)=P$.

Thus, we see that $D$ is surjective.

Use similar arguments for $D_{+}$.

For any label $\mathcal{M} \neq F I N(\mathbb{N}), \Theta(\mathcal{M})$ is a proper, closed $F I N(\mathbb{N})$ invariant subspace of $\mathcal{L} \mathcal{A B}$ and so the restriction map is a continuous, surjective monoid homomorphism from $\mathcal{E}(\mathcal{L} \mathcal{A B}) \rightarrow \mathcal{E}(\Theta(\mathcal{M}))$. We can regard it as a map from $\hat{\mathcal{E}}(\mathcal{L} \mathcal{A B})$ with $\hat{U}$ and $U$ both mapping to $U$ in $\mathcal{E}(\Theta(\mathcal{M}))$. Composing with this restriction we obtain continuous, surjective monoid homomorphisms

$$
\begin{gathered}
D, D_{+}: \beta_{b} \mathbb{Z} \rightarrow \mathcal{E}(\Theta(\mathcal{N})) \\
p x[\mathcal{N}]=x[D(p)(\mathcal{N})] \text { and } p x_{+}[\mathcal{N}]=x_{+}\left[D_{+}(p)(\mathcal{N})\right]
\end{gathered}
$$

for $\mathcal{N} \in \Theta(\mathcal{M})$.

On the other hand, the $\mathbb{Z}$ action on $X(\mathcal{M})$ extends to an Ellis action of $\phi: \beta \mathbb{Z} \times X(\mathcal{M}) \rightarrow X(\mathcal{M})$ with $\phi^{\#}: \beta Z \rightarrow X(\mathcal{M})^{X(\mathcal{M})}$ a continuous monoid homomorphism with image the enveloping semigroup $E(X(\mathcal{M}), S)$. Similarly, the action $\mathbb{Z}$ on $X_{+}(\mathcal{M})$ extends to $\phi_{+}: \beta \mathbb{Z} \times X_{+}(\mathcal{M}) \rightarrow X_{+}(\mathcal{M})$ with $\phi_{+}^{\#}(\beta \mathbb{Z})=$ $E\left(X_{+}(\mathcal{M}), S\right)$. We let

$$
E_{b}(X(\mathcal{M}), S)=\phi^{\#}\left(\beta_{b} \mathbb{Z}\right) \quad \text { and } \quad E_{b}\left(X_{+}(\mathcal{M}), S\right)=\phi_{+}^{\#}\left(\beta_{b} \mathbb{Z}\right)
$$


The maps $D$ and $D_{+}$clearly factor through $\phi^{\#}$ and $\phi_{+}^{\#}$ to define continuous, surjective homomorphisms

$$
\begin{aligned}
& D: E_{b}(X(\mathcal{M}), S) \rightarrow \mathcal{E}(\Theta(\mathcal{M})), \quad \text { and } \quad D_{+}: E_{b}\left(X_{+}(\mathcal{M}), S\right) \rightarrow \mathcal{E}(\Theta(\mathcal{M})) \\
& p x[\mathcal{N}]=x[D(p)(\mathcal{N})] \text { and } p x_{+}[\mathcal{N}]=x_{+}\left[D_{+}(p)(\mathcal{N})\right] \text {, }
\end{aligned}
$$

for $\mathcal{N} \in \Theta(\mathcal{M})$.

Definition 3.30. For $\mathcal{M} \in \mathcal{L} \mathcal{A B}$ let $\operatorname{Nuc}(X(\mathcal{M}))=x[\Theta(\mathcal{M})]$. We have

$$
\operatorname{Nuc}(X(\mathcal{M}))=E_{b}(X(\mathcal{M}), S) x[\mathcal{M}]=\{x[\mathcal{N}]: \mathcal{N} \in \Theta(\mathcal{M})\} \text {. }
$$

We call this closed, $E_{b}(X(\mathcal{M}), S)$ invariant subset, the nucleus of the labeled subshift $(X(\mathcal{M}), S)$. $\operatorname{Nuc}\left(X_{+}(\mathcal{M})\right)$ is defined analogously.

Definition 3.31. (a) If $Y$ is a subset of $\{0,1\}^{\mathbb{Z}}$ we let

$$
\Phi(Y)=\{\mathcal{N}: x[\mathcal{N}] \in Y\} \quad \text { and } \quad \Phi_{+}(Y)=\left\{\mathcal{N}: x_{+}[\mathcal{N}] \in Y\right\} .
$$

(b) If $\Psi$ is a nonempty subset of $\mathcal{L} \mathcal{A B}$ we let $\Theta(\Psi)$ denote the smallest, closed $F I N(\mathbb{N})$ invariant subset of $\mathcal{L} \mathcal{A B}$ which contains $\Psi$.

That is, $\Phi(Y)$ and $\Phi_{+}(Y)$ are the preimages of $Y$ by the maps $x[\cdot]$ and $x_{+}[\cdot]$ respectively. By Theorem $3.18 \mathcal{N}$ is uniquely determined by $x[\mathcal{N}]$ and by $x_{+}[\mathcal{N}]$.

Recall that for $\Psi$ a nonempty subset of $\mathcal{L} \mathcal{A B}, X(\Psi)$ and $X_{+}(\Psi)$ are the closed, shift invariant subsets of $\{0,1\}^{\mathbb{Z}}$ generated by the $x[\mathcal{N}]$ 's and $x_{+}[\mathcal{N}]$ 's respectively with $\mathcal{N}$ varying over $\Psi$.

Theorem 3.32. (a) If $Y$ is a nonempty, closed, shift invariant subset of $X(\mathcal{P} I P(k))$ then $\Phi(Y), \Phi_{+}(Y)$ are closed, FIN $(\mathbb{N})$ invariant subsets of $\mathcal{L} \mathcal{A B}$ with $\emptyset \in \Phi(Y) \cap \Phi_{+}(Y)$. Furthermore,

$$
X(\Phi(Y)) \cup X_{+}\left(\Phi_{+}(Y)\right) \subset Y .
$$

(b) If $\Psi$ is a nonempty subset of $\mathcal{L} \mathcal{A B}$, then

$$
X(\Psi)=X(\Theta(\Psi)) \text { and } X_{+}(\Psi)=X_{+}(\Theta(\Psi)) .
$$

(c) If $\Psi$ is a closed, $F I N(\mathbb{N})$ invariant subset of $\mathcal{L} \mathcal{A B}$ with $\emptyset \in \Psi$ then

$$
\Psi=\Phi(X(\Psi))=\Phi_{+}\left(X_{+}(\Psi)\right) .
$$

Proof: (a): Because the maps $x[\cdot], x_{+}[\cdot]$ are continuous, $\Phi(Y)$ and $\Phi_{+}(Y)$ are closed when $Y$ is. Since $I P(k)$ has Banach density zero, the fixed point $e$ is contained in $Y$ and so $x[\emptyset]=x_{+}[\emptyset]=e$ implies that $\emptyset$ is contained in $\Phi(Y)$ and $\Phi_{+}(Y)$. 
The final inclusion in (a) is obvious.

If $x[\mathcal{N}] \in Y$ (or $x_{+}[\mathcal{N}] \in Y$ ) and $\mathbf{r}$ is a nonzero $\mathbb{N}$-vector then by Lemma 3.13 we can choose a sequence $\left\{t^{i}: i \in \mathbb{N}\right\}$ in $I P_{+}(k)$ with length vector $\mathbf{r}$ and with $\left|j_{r}\left(t^{i}\right)\right| \rightarrow \infty$. By Theorem $3.25 S^{t_{i}} x[\mathcal{N}] \rightarrow x[\mathcal{N}-\mathbf{r}]\left(\right.$ or $\left.S^{t_{i}} x_{+}[\mathcal{N}] \rightarrow x_{+}[\mathcal{N}-\mathbf{r}]\right)$. Since $Y$ is closed, $\mathcal{N}-\mathbf{r} \in \Phi$ (resp. $\left.\mathcal{N}-\mathbf{r} \in \Phi_{+}\right)$. Thus, $\Phi(Y)$ and $\Phi_{+}(Y)$ are $F I N(\mathbb{N})$ invariant.

(b): With $\mathcal{N} \in \Psi$ apply the above argument to $x[\mathcal{N}] \in X(\Psi)$ we see that $x[\mathcal{N}-\mathbf{r}] \in X(\Psi)$ for all $\mathbf{r} \in F I N(\mathbb{N})$. Since $x[\cdot]$ is continuous and $X(\Psi)$ we see that $x[\mathcal{M}] \in X(\Psi)$ for all $\mathcal{M} \in \Theta(\Psi)$. Hence, $X(\Theta(\Psi)) \subset X(\Psi)$ and the reverse inclusion is obvious. The argument for $X_{+}$is similar.

(c): Clearly, $\Psi \subset \Phi(X(\Psi))$. If $\mathcal{N} \in \Phi(X(\Psi))$ with $\mathcal{N}$ nonempty, then there is a sequence $\left\{S^{t_{i}}\left(x\left[\mathcal{M}^{i}\right]\right)\right\}$ converging to $x[\mathcal{N}]$ with $\mathcal{M}^{i} \in \Psi$.

If $t^{i}=0$ infinitely often then $x[\mathcal{N}]$ is the limit of a subsequence $\left\{x\left[\mathcal{N} i^{i^{\prime}}\right]\right\}$. Then $\mathcal{N}$ is the limit of $\left\{\mathcal{N} i^{i^{\prime}}\right\}$ because $x[\cdot]$ is a homeomorphism. Since $\Psi$ is closed, $\mathcal{N} \in \Psi$.

Alternatively, we may assume that the $t^{i}$ 's are nonzero. Theorem $3.25 \mathrm{im}-$ plies that eventually $t^{i} \in A\left[\mathcal{N}^{i}\right]$ and $\mathcal{N}=\operatorname{LIM}\left\{\mathcal{M}-\mathbf{r}\left(t^{i}\right)\right\}$. Since $\Psi$ is closed and $F I N(\mathbb{N})$ invariant it follows that $\mathcal{N} \in \Psi$.

Thus, $\Phi(X(\Psi))$ is contained in and so equals $\Psi$.

Again,the argument for $X_{+}$is similar.

Corollary 3.33. (a) If a label $\mathcal{M}$ is not the maximum label $F I N(\mathbb{N})$, then

$$
\Phi(X(\mathcal{M}))=\Phi_{+}\left(X_{+}(\mathcal{M})\right)=\Theta(\mathcal{M}) .
$$

(b) For $F I N(\mathbb{N})$, the maximum label, $\Theta(F I N(\mathbb{N}))=\{F I N(\mathbb{N})\}$ and

$$
\begin{gathered}
\Phi(X(F I N(\mathbb{N})))=\Phi_{+}\left(X_{+}(F I N(\mathbb{N}))\right)= \\
\Theta(F I N(\mathbb{N})) \cup\{\emptyset\}=\{F I N(\mathbb{N}), \emptyset\} .
\end{gathered}
$$

Proof: (a): From Theorem 3.32(b) $X(\mathcal{M})=X(\Theta(\mathcal{M}))$. Since $\mathcal{M} \neq$ $F I N(\mathbb{N})$, there exists $\mathbf{r} \in F I N(\mathbb{N}) \backslash \mathcal{M}$ and so $\mathcal{M}-\mathbf{r}=\emptyset$. Hence, $\emptyset \in \Theta(\mathcal{M})$. Thus, Theorem 3.32(c) implies that $\Phi(X(\Theta(\mathcal{M})))=\Theta(\mathcal{M})$.

(b): For $F I N(\mathbb{N})$, the maximum label, $F I N(\mathbb{N})-\mathbf{r}=F I N(\mathbb{N})$ for all $\mathbf{r} \in$ $F I N(\mathbb{N})$ and so $\Theta(F I N(\mathbb{N}))=\{F I N(\mathbb{N})\}$. In particular, $\emptyset \notin \Theta(F I N(\mathbb{N}))$.

Hence, $\Psi=\{F I N(\mathbb{N}), \emptyset\}$ is closed, $F I N(\mathbb{N})$ invariant and contains $\emptyset$.

Clearly, $\Phi(X(F I N(\mathbb{N})))=\Phi(X(\Psi))$ which equals $\Psi$ by Theorem 3.32(c) again.

The similar arguments for $\Phi_{+}$are left to the reader. 
Theorem 3.34. Let $\mathcal{M}$ be a label of finite type with $(X(\mathcal{M}), S)$ and $\left(X_{+}(\mathcal{M}), S\right)$ the associated subshifts.

(a)

$$
X(\mathcal{M})=\left\{S^{k}(x[\mathcal{N}]): k \in \mathbb{Z}, \mathcal{N} \in \Theta(\mathcal{M})\right\}=\bigcup_{k \in \mathbb{Z}} S^{k} \operatorname{Nuc}(X(\mathcal{M}))
$$

and

$$
X_{+}(\mathcal{M})=\left\{S^{k}\left(x_{+}[\mathcal{N}]\right): k \in \mathbb{Z}, \mathcal{N} \in \Theta(\mathcal{M})\right\}=\bigcup_{k \in \mathbb{Z}} S^{k} \operatorname{Nuc}\left(X_{+}(\mathcal{M})\right)
$$

(b) If $Y$ is a nonempty, closed, shift invariant subset of $X(\mathcal{M})$ then

$$
Y=X(\Phi(Y))=\left\{S^{k}(x[\mathcal{N}]): k \in \mathbb{Z}, \mathcal{N} \in \Phi(Y)\right\}
$$

(c) If $Y$ is a nonempty, closed, shift invariant subset of $X_{+}(\mathcal{M})$ then

$$
Y=X_{+}\left(\Phi_{+}(Y)\right)=\left\{S^{k}\left(x_{+}[\mathcal{N}]\right): k \in \mathbb{Z}, \mathcal{N} \in \Phi_{+}(Y)\right\}
$$

Proof: (a): Let $z \in X(\mathcal{M})$.

If $z=e$ then $z=x[\emptyset]$ and $\emptyset \in \Theta(\mathcal{M})$ since $\mathcal{M} \neq \operatorname{FIN}(\mathbb{N})$.

Assume that $z \neq e$ and so $z=S^{k}(w)$ with $w_{0}=1$. Since $w \in X(\mathcal{M})$ it is the limit of $\left\{S^{t^{i}}(x[\mathcal{M}])\right\}$ for some sequence $\left\{t^{i}\right\}$ in $\mathbb{Z}$. Eventually $t^{i} \in A[\mathcal{M}]$ and so we may assume that $t^{i} \in A[\mathcal{M}]$ for all $i$.

If some subsequence of $\left\{t^{i}\right\}$ is bounded then there exists $k_{1} \in \mathbb{Z}$ such that $t^{i}=k_{1}$ infinitely often and so $w=S^{k_{1}}(x[\mathcal{M}])$.

Now assume that $\left|t^{i}\right| \rightarrow \infty$ and $t^{i} \neq 0$ for all $i$.

If $n_{*}\left(t^{i}\right)=\left|j_{r\left(t^{i}\right)}\left(t^{i}\right)\right| \rightarrow \infty$ then by Theorem $3.25 w=x[\mathcal{N}]$ with $\mathcal{N} \in \Theta^{\prime}(\mathcal{M})$.

Notice that these results only require $\mathcal{M} \neq F I N(\mathbb{N})$.

Now assume that $\left\{\left|j_{r\left(t^{i}\right)}\left(t^{i}\right)\right|\right\}$ has a bounded subsequence. By Lemma 3.21 we may go to a subsequence $\left\{t^{i^{\prime}}\right\}$ so that $t^{i^{\prime}}=\tilde{t}^{i^{\prime}}+\bar{t}$ with $\tilde{t}^{i^{\prime}} \in A[\mathcal{M}]$ and $n_{*}\left(\tilde{t}^{i^{\prime}}\right) \rightarrow \infty$. By Theorem 3.25 again $\left\{S^{\tilde{t}^{i^{\prime}}}(x[\mathcal{M}])\right\}$ has limit $x[\mathcal{N}]$ for some $\mathcal{N} \in \Theta(\mathcal{M})$. Since $\bar{t}$ is constant, $w=\operatorname{Lim}\left\{S^{t^{i^{\prime}}}(x[\mathcal{M}])\right\}=S^{\bar{t}}(x[\mathcal{N}])$.

Thus, in any of these cases $w=S^{k_{2}}(x[\mathcal{N}])$ for some $k_{2} \in \mathbb{Z}$ and $\mathcal{N} \in \Theta(\mathcal{M})$. Finally $z=S^{k+k_{2}}(x[\mathcal{N}])$ as required.

The argument for $z \in X_{+}(\mathcal{M})$ is similar. If $w \in X_{+}(\mathcal{M})$ with $w_{0}=1$ then we can assume that $t^{i} \in A_{+}[\mathcal{M}]$ for all $i$ and proceed as above.

(b), (c): If $Y$ is an invariant subset of $X(\mathcal{M})$ then $Y$ consists of the orbits of some of these $x[\mathcal{N}]$. That is, $\Phi(Y) \subset \Theta(\mathcal{M})$ and $Y$ consists of the orbits of the points $x[\mathcal{N}]$ for $\mathcal{N} \in \Phi(Y)$. Thus, $Y=X(\Phi(Y))$. The $X_{+}$case in (c) is similar.

Corollary 3.35. Given $\mathcal{M} \in \mathcal{L} \mathcal{A B}$ : 
(1) The map $\Psi \mapsto X(\Psi)$ is one-to-one from the collection of closed $F I N(\mathbb{N})$ invariant subsets of $\Theta(\mathcal{M})$ to collection of closed $S$-invariant subsets of $X(\mathcal{M})$.

(2) If $\mathcal{M}$ is of finite type then this map is surjective, i.e. every closed $S$-invariant subsets $Y$ of $X(\mathcal{M})$ is of the form $X(\Psi)$ for some closed $F I N(\mathbb{N})$ invariant $\Psi \subset \Theta(\mathcal{M})$, with

$$
\Phi(X(\Psi))=\Psi \quad \text { and } Y=X(\Phi(Y)) .
$$

Similar statements hold for $X_{+}(\mathcal{M})$.

Proof: Combine Theorem 3.32 (c) and Theorem 3.34 (b).

Corollary 3.36. If a label $\mathcal{M}$ is not of finite type then $X(\mathcal{M})$ and $X_{+}(\mathcal{M})$ each contain non-periodic recurrent points.

If a label $\mathcal{M}$ is of finite type then $e$ is the only recurrent point of $X(\mathcal{M})$ or $X_{+}(\mathcal{M})$ and so $(X(\mathcal{M}), S)$ and $\left(X_{+}(\mathcal{M}), S\right)$ are $C T$ systems. In that case, $(X(\mathcal{M}), S)$ and $\left(X_{+}(\mathcal{M}), S\right)$ are LE and topologically transitive but not weak mixing.

Proof: If $\mathcal{M}$ is not of finite type then Proposition 2.25 implies that there is a positive recurrent label $\mathcal{N}$ with $\mathcal{N} \in \Theta(\mathcal{M})$. Hence, $x[\mathcal{N}] \neq e$ is a recurrent point of $X(\mathcal{M})$ by Corollary 3.26.

If $\mathcal{M}$ is of finite type then by Corollary 3.34 every point of $X(\mathcal{M})$ is on the orbit of some $x\left[\mathcal{M}_{1}\right]$ with $\mathcal{M}_{1} \subset \mathcal{M}$. These are all labels of finite type and so none is recurrent except for $\mathcal{M}_{1}=\emptyset$.

Since $x[\mathcal{M}]$ is always a transitive point for $X(\mathcal{M}),(X(\mathcal{M}), S)$ is always topologically transitive. In the finite type case, it is CT and so is LE and not weak mixing (see Remarks 1.15 and 1.25).

Corollary 3.37. Assume that $\mathcal{M}_{1}$ and $\mathcal{M}_{2}$ are labels with $\mathcal{M}_{1}$ of finite type. The subshifts $\left(X\left(\mathcal{M}_{1}\right), S\right)$ and $\left(X\left(\mathcal{M}_{1}\right), S\right)$ are isomorphic only if $\mathcal{M}_{1}=\mathcal{M}_{2}$.

Proof: The result is clearly true if each of $\mathcal{M}_{1}$ and $\mathcal{M}_{2}$ is either 0 or $\emptyset$. If $\mathcal{M}_{2}$ is not of finite type then by Corollary $3.36 X\left(\mathcal{M}_{2}\right)$ and $X_{+}\left(\mathcal{M}_{2}\right)$ contain non-trivial recurrent points while $X\left(\mathcal{M}_{1}\right)$ and $X_{+}\left(\mathcal{M}_{1}\right)$ do not.

We may restrict to the case where both $\mathcal{M}_{1}$ and $\mathcal{M}_{2}$ are of finite type with $\mathbf{r}>\mathbf{0}$ in $\mathcal{M}_{1} \backslash \mathcal{M}_{2}$. By Lemma 3.13 we can choose a sequence $\left\{t^{n} \in I P_{+}(k)\right\}$ so that $\mathbf{r}\left(t^{n}\right)=\mathbf{r}$ and $j_{r}\left(t^{n}\right)>n$. Go to a subnet $\left\{t^{n_{i}}\right\}$ converging to $p \in \beta \mathbb{Z}$. By the Coherence Lemma 3.20 for any label $\mathcal{M}, p(x[\mathcal{N}])=x[\mathcal{M}-\mathbf{r}]$ and $p\left(x_{+}[\mathcal{M}]\right)=x_{+}[\mathcal{M}-\mathbf{r}]$. In particular, since $\mathcal{M}_{2}-\mathbf{r}=\emptyset, p(x[\mathcal{N}])=p\left(x_{+}[\mathcal{N}]\right)=e$ for every label $\mathcal{N} \subset \mathcal{M}_{2}$. Because $\mathcal{M}_{2}$ is of finite type, it follows from Theorem 
3.34(a) that $p(x)=e$ for all $x \in X\left(\mathcal{M}_{2}\right)$ and all $x \in X_{+}\left(\mathcal{M}_{2}\right)$. On the other hand, $\mathcal{M}_{1}-\mathbf{r}$ is nonempty and so $p(x[\mathcal{N}])$ and $p\left(x\left[\mathcal{M}_{1}\right]\right)$ and $p\left(x_{+}\left[\mathcal{M}_{1}\right]\right)$ are not equal to $e$. Since $e$ is the unique minimal point in any $X(\mathcal{M})$ it follows that $\{p: p(x)=e\}$ for all $x \in X(\mathcal{M})\}$ is an isomorphism invariant for the cascade $(X(\mathcal{M}), S)$. It follows that $\left(X\left(\mathcal{M}_{1}\right), S\right)$ is not isomorphic to $\left(X\left(\mathcal{M}_{2}\right), S\right)$.

Question 3.38. For distinct recurrent labels $\mathcal{M}_{1}$ and $\mathcal{M}_{2}$ can it happen that the subshifts $\left(X\left(\mathcal{M}_{1}\right), S\right)$ and $\left(X\left(\mathcal{M}_{2}\right), S\right)$ are isomorphic?

Example 3.39. For the finite type label $\mathcal{M}$ in Example $2.29 \Theta(\mathcal{M})$ is uncountable and so the subshift $X=X(\mathcal{M})$ is uncountable, but nonetheless CT and LE. In fact, each point of $X$ is an isolated point in its orbit closure, and $e$ is the unique recurrent point. Thus $\mathcal{M}$ is a finite type label with uncountable $X(\mathcal{M})$.

Define

$$
\begin{array}{r}
S Y M=\left\{x \in\{0,1\}^{\mathbb{Z}}: x_{0}=1 \text { and } x_{-t}=x_{t} \text { for all } t \in \mathbb{Z}\right\}, \\
Z E R=\left\{x \in\{0,1\}^{\mathbb{Z}}: x_{0}=1 \text { and } x_{t}=0 \text { for all } t<0\right\} .
\end{array}
$$

Lemma 3.40. SYM and $Z E R$ are closed subsets of $\{0,1\}^{\mathbb{Z}}$.

$$
\begin{array}{r}
S Y M \cap X(\mathcal{L A B})=x[\mathcal{L A B}] \backslash\left\{e=x_{+}[\emptyset]\right\} ; \\
Z E R \cap X_{+}(\mathcal{L} \mathcal{A B})=x_{+}[\mathcal{L} \mathcal{A B}] \backslash\left\{e=x_{+}[\emptyset]\right\} .
\end{array}
$$

Each non-periodic $S$ orbit in $\{0,1\}^{\mathbb{Z}}$ meets $S Y M$ at most once. Each $S$ orbit in $\{0,1\}^{\mathbb{Z}}$ meets $Z E R$ at most once

Proof: $S Y M$ and ZER are obviously closed. Equation 3.38 follows from Theorem 3.25.

If $S^{k_{1}} x, S^{k_{2}} x \in S Y M$ with $k_{2} \neq k_{1}$ then for all $t \in \mathbb{Z}$,

$$
x_{t+k_{1}}=x_{-t+k_{1}}=x_{\left(-t+k_{1}-k_{2}\right)+k_{2}}=x_{\left(t-k_{1}+k_{2}\right)+k_{2}}
$$

Letting $s=t+k_{1}$ we have that $x_{s}=x_{s+2\left(k_{2}-k_{1}\right)}$ for all $s \in \mathbb{Z}$. Since $k_{2} \neq k_{1}$ it follows that $x$ is periodic. By Corollary $3.17 e=x[\emptyset]$ is the only periodic point in $X(\mathcal{P} I P(k))$.

The result for $Z E R$ is obvious. 
Remark 3.41. It follows that if $\mu$ is any non-atomic, shift-invariant probability measure on $\{0,1\}^{\mathbb{Z}}$ then $\mu(Z E R)=\mu(S Y M)=0$. Observe first that the countable set $P E R$ of periodic points in $\{0,1\}^{\mathbb{Z}}$ has measure zero because $\mu$ is non-atomic. Since $\left\{S^{k}(S Y M \backslash P E R): k \in \mathbb{Z}\right\}$ and $\left\{S^{k}(Z E R): k \in \mathbb{Z}\right\}$ are pairwise disjoint sequences of sets with identical measure the common value must be zero.

Proposition 3.42. Let $x^{*}$ be a non-periodic recurrent point of $\{0,1\}^{\mathbb{Z}}$ and let $X$ be its orbit closure, so that $(X, S)$ is the closed subshift generated by $x^{*}$. If $K$ is a closed subset of $X$ such that every non-periodic orbit in $X$ meets $K$ at most once, then $K$ is nowhere dense. The set $X \backslash \bigcup_{k \in \mathbb{Z}}\left\{S^{-k}(K)\right\}$ is a dense $G_{\delta}$ subset of $X$.

In particular, SYM $\cap X$ is nowhere dense in $X$ and the set of points of $X$ whose orbit does not meet $S Y M$ is a dense $G_{\delta}$ subset of $X$. Similarly, $Z E R \cap X$ is nowhere dense in $X$ and the set of points of $X$ whose orbit does not meet $Z E R$ is a dense $G_{\delta}$ subset of $X$.

Proof: Since the orbit of $x^{*}$ is dense in $X$, it meets any nonempty open subset of $X$. If the interior of $K$ contained more than one point then there would be two disjoint open sets $U_{1}, U_{2}$ contained in $K$ and so there would exist $k_{1}, k_{2} \in \mathbb{Z}$ such that $S^{k_{a}} x^{*} \in U_{a} \subset K$ for $a=1,2$. Since $U_{1}$ and $U_{2}$ are disjoint $k_{2} \neq k_{1}$. This contradicts the assumption on $K$. Hence, if $K$ has nonempty interior then the interior consists of a single point which is on the orbit of $x^{*}$. This would imply that $x^{*}$ is an isolated point and so cannot be recurrent unless it is periodic. Hence, the interior of $K$ is empty.

Since $K$ is nowhere dense, the $G_{\delta}$ set $X \backslash \bigcup_{k \in \mathbb{Z}}\left\{S^{-k}(K)\right\}$ is dense by the Baire Category Theorem. A point lies in this set exactly when its orbit does not meet $K$.

The result applies to $K=S Y M \cap X$ by Lemma 3.40 .

In the case of $Z E R$ the result is clear because the $S^{-1}$ transitive points in the orbit closure of $x^{*}$ form a dense $G_{\delta}$ set disjoint from $Z E R$.

Corollary 3.43. For any label $\mathcal{M}$, the set $\{e\} \cup X(\mathcal{M}) \cap S Y M=x[\Theta(\mathcal{M})]=$ $\operatorname{Nuc}(X(\mathcal{M}))$, the nucleus of $X(\mathcal{M})$, is a compact subset of $X(\mathcal{M})$ which meets each orbit in at most one point. The set $\{e\} \cup X_{+}(\mathcal{M}) \cap Z E R=x_{+}[\Theta(\mathcal{M})]$ is a compact subset of $X_{+}(\mathcal{M})$ which meets each orbit in at most one point.

If $\mathcal{M}$ is of finite type then $x[\Theta(\mathcal{M})]$ meets each orbit in $X(\mathcal{M})$ and $x_{+}[\Theta(\mathcal{M})]$ meets each orbit in $X_{+}(\mathcal{M})$.

If $\mathcal{M}$ is not of finite type then $X(\mathcal{M}) \backslash \bigcup S^{i}(S Y M)$ is non-empty and so $\left\{S^{k} x[\mathcal{N}]: k \in \mathbb{Z}, \mathcal{N} \in \Phi(X(\mathcal{M}))\right\}$ is a proper subset of $X(\mathcal{M})$. Furthermore, 
$X_{+}(\mathcal{M}) \backslash\left[\{e\} \cup \cup S^{i}(Z E R)\right]$ is non-empty and so $\left\{S^{k}\left(x_{+}[\mathcal{N}]\right): k \in \mathbb{Z}, \mathcal{N} \in\right.$ $\left.\Phi_{+}\left(X_{+}(\mathcal{M})\right)\right\}$ is a proper subset of $X_{+}(\mathcal{M})$.

Proof: By Lemma 3.40 a non-periodic orbit meets $S Y M$ in at most one point. $X(\mathcal{M})$ is compact and $S Y M$ is closed and so the intersection is compact. $x[\Theta(\mathcal{M})] \subset X(\mathcal{M})$ and $x[\mathcal{L} \mathcal{A B}] \subset S Y M$. On the other hand, by Theorem 3.25 if $z \in X(\mathcal{M}) \cap S Y M$ then $z=x[\mathcal{N}]$ for some $\mathcal{N} \in \Theta(\mathcal{M})$.

If $\mathcal{M}$ is of finite type then each orbit of $X(\mathcal{M})$ meets $x[\Theta(\mathcal{M})]$.

If $\mathcal{M}$ is not of finite type then by Corollary 3.36there exists a non-periodic recurrent point $x^{*} \in X(\mathcal{M})$. If $X^{*}$ is the orbit closure of $x^{*}$ then $X^{*} \subset X(\mathcal{M})$ and by Proposition $3.42 X^{*} \backslash \bigcup S^{i}(S Y M)$ is nonempty.

Remark 3.44. Corollary 3.43 shows that for any label $\mathcal{M}$ of finite type the dynamical system $(X(\mathcal{M}), S)$ admits $\operatorname{Nuc}(X(\mathcal{M}))=x[\Theta(\mathcal{M})]$ as a closed crosssection; i.e. the orbit of any point $x \in X(\mathcal{M})$ meets $x[\Theta(\mathcal{M})]$ exactly at one point. This is in accordance with the following general theorem (see [27, Section 1.2]).

Theorem 3.45. For a system $(X, T)$, with $X$ a completely metrizable separable space, there exists a Borel cross-section if and only if the only recurrent points are the periodic ones.

Notice, too, that if $\mu$ is an invariant probability measure on $(X, T)$ such that the measure of the set of periodic points is zero, then any cross-section is a non-measurable set. The special case of translation by rationals on $\mathbb{R} / \mathbb{Z}$ is used in the usual proof of the existence of a subset of $\mathbb{R}$ which is not Lebesgue measurable.

On the other hand, when $\mathcal{M}$ is recurrent, $\operatorname{Nuc}(X(\mathcal{M}))=x[\Theta(\mathcal{M})]$ is a Cantor subset of $X(\mathcal{M})$ which meets each orbit at most once. Thus, $S^{i}(x[\Theta(\mathcal{M})]) \cap$ $S^{j}(x[\Theta(\mathcal{M})])=\emptyset$ whenever $i \neq j$ in $\mathbb{Z}$. While this explicit construction may be of interest, in fact any system $(X, T)$ admits such wandering Cantor sets when $X$ is perfect and the set of periodic points has empty interior, see [3, Theorem 1.4] .

4. WAP LABELS AND THEIR SUBSHIFTS

\subsection{Simple, semi-simple and finitary labels.}


Since for any label $\mathcal{M}, \Theta(\mathcal{M})$ is a closed $F I N(\mathbb{N})$ invariant set, the enveloping semigroup $\mathcal{E}(\Theta(\mathcal{M}))$ was defined above as the pointwise closure of the set of maps $P_{\mathbf{r}}$ on $\Theta(\mathcal{M})$ and the adherence semigroup $\mathcal{A}(\Theta(\mathcal{M}))$ as the closure of the subset of maps $P_{\mathbf{r}}$ with $\mathbf{r}>0$. Of course, $P_{\mathbf{0}}$ is the identity on $\Theta(\mathcal{M})$. We have

$$
\mathcal{E}(\Theta(\mathcal{M})) \cdot \mathcal{M}=\Theta(\mathcal{M}), \quad \text { and } \quad \mathcal{A}(\Theta(\mathcal{M})) \cdot \mathcal{M}=\Theta^{\prime}(\mathcal{M})
$$

Recall that $\mathcal{N} \in \Theta^{\prime}(\mathcal{M})$ implies $\max \mathcal{M} \cap \mathcal{N}=\emptyset$, so if $\mathbf{r} \in \max \mathcal{M}$ then for $\mathcal{N} \in \Theta(\mathcal{M}):$

$$
P_{\mathbf{r}}(\mathcal{N})= \begin{cases}0 & \text { if } \mathcal{N}=\mathcal{M}, \\ \emptyset & \text { if } \mathcal{N} \in \Theta^{\prime}(\mathcal{M}) .\end{cases}
$$

Lemma 4.1. If $\mathcal{M}$ is a bounded, nonempty label then $\mathcal{A}(\Theta(\mathcal{M}))$ is a closed subsemigroup which equals $\mathcal{E}(\Theta(\mathcal{M})) \backslash\left\{P_{\mathbf{0}}\right\}$. If $Q \in \mathcal{A}(\Theta(\mathcal{M})$ then $Q(0)=\emptyset$.

Proof: Since $\mathcal{M}$ is nonempty and bounded, it follows from Lemma 2.18 that $0 \in \Theta(\mathcal{M})$. The set of elements $Q \in \mathcal{E}(\Theta(\mathcal{M}))$ such that $Q(0)=\emptyset$ is a closed subsemigroup which contains all $P_{\mathbf{r}}$ for $\mathbf{r}>0$. So it contains $\mathcal{A}(\Theta(\mathcal{M})$. The sole remaining point $P_{\mathbf{0}}$ has $P_{\mathbf{0}}(0)=0$ and so is not in $\mathcal{A}(\Theta(\mathcal{M}))$.

In this section we will examine conditions on a label $\mathcal{M}$ which ensure that the action of $F I N(\mathbb{N})$ on $\Theta(\mathcal{M})$ is WAP, that is, every element of the enveloping semigroup $\mathcal{E}(\Theta(\mathcal{M}))$ is continuous on $\Theta(\mathcal{M})$. By Corollary 1.9 this is equivalent to commutativity of the monoid $\mathcal{E}(\Theta(\mathcal{M}))$. In that case, we will say that the label $\mathcal{M}$ is WAP.

Definition 4.2. A label $\mathcal{M}$ is WAP if every element of the enveloping semigroup $\mathcal{E}(\Theta(\mathcal{M}))$ is continuous on $\Theta(\mathcal{M})$

We will denote by $\mathcal{E}_{0}(\Theta(\mathcal{M}))$ the elements which come from $F I N(\mathbb{N})$. That is,

$$
\varepsilon_{0}(\Theta(\mathcal{M}))=\left\{P_{\mathbf{r}} \mid \Theta(\mathcal{M}): \mathbf{r} \in F I N(\mathbb{N})\right\}
$$

We will write $P_{\mathbf{r}}$ in $\mathcal{E}(\Theta(\mathcal{M}))$ for the restriction $P_{\mathbf{r}} \mid \Theta(\mathcal{M})$.

We call the members of the set

$$
\varepsilon_{*}(\Theta(\mathcal{M}))=\mathcal{E}(\Theta(\mathcal{M})) \backslash \mathcal{E}_{0}(\Theta(\mathcal{M}))
$$

the external elements of $\mathcal{E}(\Theta(\mathcal{M}))$.

Lemma 4.3. Let $\mathcal{M}$ be a label.

(a) In $\mathcal{E}_{0}(\Theta(\mathcal{M}))$ the map $P_{\mathbf{0}}$ is the identity map on $\Theta(\mathcal{M})$ and so is the identity element of the monoid $\mathcal{E}(\Theta(\mathcal{M}))$. If $\mathcal{M}=F I N(\mathbb{N})$ then $\Theta(\mathcal{M})=\{\mathcal{M}\}$ and $\mathcal{E}(\Theta(\mathcal{M}))$ is the trivial monoid $\left\{P_{\mathbf{0}}\right\}$. If $\mathbf{r} \notin \mathcal{M}$ then $P_{\mathbf{r}}=U$ the map taking all of $\Theta(\mathcal{M})$ to $\emptyset \in \Theta(\mathcal{M})$. 
(b) If $\mathcal{M}-\mathbf{r}_{1}=\mathcal{M}-\mathbf{r}_{2}$ for some $\mathbf{r}_{1}, \mathbf{r}_{2} \in F I N(\mathbb{N})$ then $P_{\mathbf{r}_{1}} \mid \Theta(\mathcal{M})=$ $P_{\mathbf{r}_{2}} \mid \Theta(\mathcal{M})$, i.e. as elements of $\mathcal{E}_{0}(\Theta(\mathcal{M})), P_{\mathbf{r}_{1}}=P_{\mathbf{r}_{2}}$.

(c) If $Q_{1} \in \mathcal{E}\left(\Theta(\mathcal{M})\right.$ ) satisfies $Q_{1}(\mathcal{M})=\mathcal{M}-\mathbf{r}_{1}$ for some $\mathbf{r}_{1} \in F \operatorname{IN}(\mathbb{N})$ then $Q_{1}(\mathcal{M}-\mathbf{r})=\mathcal{M}-\mathbf{r}_{1}-\mathbf{r}$ for all $\mathbf{r} \in F I N(\mathcal{M})$. If, in addition, $Q_{2} \in \mathcal{E}(\Theta(\mathcal{M}))$ satisfies $Q_{2}(\mathcal{M})=\mathcal{M}-\mathbf{r}_{2}$ for some $\mathbf{r}_{2} \in F I N(\mathbb{N})$ then $Q_{1} Q_{2}(\mathcal{M})=Q_{2} Q_{1}(\mathcal{M})=\mathcal{M}-\mathbf{r}_{1}-\mathbf{r}_{2}$.

Proof: (a): Obvious.

(b): If $P_{\mathbf{r}_{1}}(\mathcal{M})=P_{\mathbf{r}_{2}}(\mathcal{M})$ then because $F I N(\mathbb{N})$ is commutative, it follows that $P_{\mathbf{r}_{1}}=P_{\mathbf{r}_{2}}$ on $F I N(\mathbb{N}) \cdot \mathcal{M}=\{\mathcal{M}-\mathbf{r}: \mathbf{r} \in F I N(\mathbb{N})\}$. Because $P_{\mathbf{r}_{1}}$ and $P_{\mathbf{r}_{2}}$ are continuous and $F I N(\mathbb{N}) \cdot \mathcal{M}$ is dense in $\Theta(\mathcal{M})$ it follows that they agree on $\Theta(\mathcal{M})$.

(c): $Q_{1} P_{\mathbf{r}}(\mathcal{M})=P_{\mathbf{r}} Q_{1}(\mathcal{M})$ implies the first result. The second is then obvious.

Let $\mathcal{L} \mathcal{A} \mathcal{B}_{f}$ denote the set of finite labels, which comprise a countable dense subset of $\mathcal{L} \mathcal{A B}$.

Definition 4.4. Let $\mathcal{M}$ be a label.

(a) The label $\mathcal{M}$ is of strong finite type if $\mathcal{M}$ is bounded and $\Theta(\mathcal{M}) \subset$ $\mathcal{L} \mathcal{A B} \mathcal{B}_{f} \cup F I N(\mathbb{N}) \cdot \mathcal{M}$. That is, if $\mathcal{N} \in \Theta(\mathcal{M})$ then either $\mathcal{N}=\mathcal{M}-\mathbf{r}$ for some $\mathbf{r} \in F I N(\mathbb{N})$ or $\mathcal{N}$ is a finite label.

(b) The label $\mathcal{M}$ is semi-simple if $Q \in \mathcal{E}_{*}(\Theta(\mathcal{M}))$ implies $Q(\mathcal{M})$ is finite.

(c) The label $\mathcal{M}$ is simple if $\Theta(\mathcal{M})=F I N(\mathbb{N}) \cdot \mathcal{M}$.

Since $\mathcal{L} \mathcal{A} \mathcal{B}_{f}$ and $F I N(\mathbb{N})$ are countable, $\Theta(\mathcal{M})$ is countable when $\mathcal{M}$ is strong finite type. It follows from Corollary 2.26 that if $\mathcal{M}$ is strong finite type then, as expected, it is of finite type. Since every element of $\Theta(\mathcal{M})$ can be obtained as $Q(\mathcal{M})$ for some $Q \in \mathcal{E}(\Theta(\mathcal{M}))$ it is clear that a bounded, semisimple label is of strong finite type. As we will later see, there exist strong finite type labels which do not satisfy monoid condition of semi-simplicity. However, in the simple case the a priori stronger monoid condition is implied.

Lemma 4.5. If $\mathcal{M}$ is a simple label then $\mathcal{E}(\Theta(\mathcal{M}))=\mathcal{E}_{0}(\Theta(\mathcal{M}))$ and so $\varepsilon_{*}(\Theta(\mathcal{M}))=\emptyset$.

Proof: Let $Q \in \mathcal{E}(\Theta(\mathcal{M}))$. By simplicity, there exists $\mathbf{r}_{1} \in F I N(\mathbb{N})$ such that $Q(\mathcal{M})=P_{\mathbf{r}}(\mathcal{M})$. By Lemma $4.3(\mathrm{c}), Q=P_{\mathbf{r}}$ on $F I N(\mathbb{N}) \cdot \mathcal{M}$. By simplicity this is all of $\Theta(\mathcal{M})$. That is, $Q=P_{\mathbf{r}}$ as elements of $\mathcal{E}(\Theta(\mathcal{M}))$.

In the strong finite type case, it may happen that $Q(\mathcal{M})=P_{\mathbf{r}}$ on $\mathcal{M}$ and so on $F I N(\mathbb{N}) \cdot \mathcal{M}$ but not on all of $\Theta(\mathcal{M})$. 
We now show that a semi-simple label is WAP.

We say that a net in $F I N(\mathbb{N}),\left\{\mathbf{r}^{i}: i \in I\right\}$, is cofinal constant if there exists $\mathbf{r} \in F I N(\mathbb{N})$ such that $\left\{i \in I: \mathbf{r}^{i}=\mathbf{r}\right\}$ is a cofinal subset of the directed set $I$.

Lemma 4.6. Assume that $\left\{\mathbf{r}^{i}: i \in I\right\}$ is a net of elements of $F I N(\mathbb{N})$.

(a) If $\left\{\mathbf{r}^{i}: i \in I\right\}$ is not cofinal constant and $\mathcal{N}$ is a finite label then eventually $\mathcal{N}-\mathbf{r}^{i}=\emptyset$.

(b) Assume $\mathcal{M}$ is a bounded label and $\mathbf{r}^{i} \in \mathcal{M}$ for all $i$. If there exists a cofinal $I^{\prime} \subset I$ such that $\bigcup\left\{\right.$ supp $\left.\mathbf{r}^{i}: i \in I^{\prime}\right\}$ is finite then the net $\left\{\mathbf{r}^{i}: i \in I\right\}$ is cofinal constant.

Proof: (a): Either $\left\{i: \mathbf{r}^{i} \in \mathcal{N}\right\}$ is cofinal or else eventually $\mathbf{r}^{i} \notin \mathcal{N}$. Let $\mathcal{N}=\left\{\mathbf{s}_{1}, \ldots, \mathbf{s}_{k}\right\}$. Let $I_{j}=\left\{i: \mathbf{r}^{i}=\mathbf{s}^{j}\right\}$. If $\left\{i: \mathbf{r}^{i} \in \mathcal{N}\right\}=\bigcup_{j} I_{j}$ is cofinal then some $I_{j}$ is cofinal and so the net is cofinal constant.

(b): Since $\mathcal{M}$ is bounded, the set $A=\left\{\mathbf{r} \in \mathcal{M}:\right.$ supp $\mathbf{r} \subset \bigcup\left\{\right.$ supp $\left.\mathbf{r}^{i}: i \in I^{\prime}\right\}$ is finite. Apply (a) to $\mathcal{N}$, the label generated by $A$. If the net were not cofinal constant then eventually $\mathbf{r}^{i} \notin \mathcal{N}$ contradicting cofinality of $I^{\prime}$.

Theorem 4.7. If $\mathcal{M}$ is a semi-simple label then $\mathcal{M}$ is WAP. If $Q_{1}, Q_{2} \in$ $\mathcal{E}_{*}(\Theta(\mathcal{M}))$ then $Q_{1} Q_{2}=Q_{2} Q_{1}=U$.

Proof: Let $\left\{\mathbf{r}^{i}: i \in I\right\}$ be a net in $F I N(\mathbb{N})$ such that $\left\{P_{\mathbf{r}^{i}}: i \in I\right\}$ converges pointwise to $Q$.

Case 1: $I^{\prime}=\left\{i: \mathbf{r}^{i} \notin \mathcal{M}\right\}$ is cofinal. For any $\mathcal{N} \in \Theta(\mathcal{N}), \mathcal{N} \subset \mathcal{M}$ and so $\mathcal{N}-\mathbf{r}^{i}=\emptyset$ for all $i \in I^{\prime}$. Hence, the subnet $\left\{P_{\mathbf{r}^{i}}: i \in I^{\prime}\right\}$ converges $U$ on $\Theta(\mathcal{M})$. Thus, $Q=U$ and so $Q P=U$ for all $P \in \mathcal{E}(\Theta(\mathcal{M}))$.

Case 2: $\left\{\mathbf{r}^{i}: i \in I\right\}$ is cofinal constant. So there exists $\mathbf{r}$ such that $\mathbf{r}^{i}=\mathbf{r}$ on a cofinal subset. Hence, $Q=P_{\mathbf{r}} \in \mathcal{E}_{0}(\Theta(\mathcal{M}))$.

Case 3: $\left\{\mathbf{r}^{i}: i \in I\right\}$ is a net in $\mathcal{M}$ which is not cofinal constant. If $\mathcal{N}$ is in the image of an external element of $\mathcal{E}(\Theta(\mathcal{M}))$ then $\mathcal{N}$ is finite since $\mathcal{M}$ is semi-simple, and so $\mathcal{N}-\mathbf{r}^{i}$ is eventually empty. So $Q(\mathcal{N})=\emptyset$.

So if $Q_{1}, Q_{2} \in \mathcal{E}_{*}(\Theta(\mathcal{M}))$ then Case 3 applies with $Q=Q_{1}$ and so $Q_{1} Q_{2}=U$.

It follows that all of the elements of $\mathcal{E}_{*}(\Theta(\mathcal{M}))$ commute with one another. Since the elements of $\mathcal{E}_{0}(\Theta(\mathcal{M}))$ commute with every element of $\mathcal{E}(\Theta(\mathcal{M}))$, it follows that $\mathcal{E}(\Theta(\mathcal{M}))$ is commutative and so $\mathcal{M}$ is WAP.

Corollary 4.8. A bounded label $\mathcal{M}$ is semi-simple iff it is strong finite type and WAP.

Proof: Assume $\mathcal{M}$ is strong finite type and WAP. If $Q(\mathcal{M})=\mathcal{M}-\mathbf{r}$ then by Lemma 4.3 (c), $Q=P_{\mathbf{r}}$ on $F I N(\mathbb{N}) \cdot \mathcal{M}$ which is dense in $\Theta(\mathcal{M})$. Since $\mathcal{M}$ 
is WAP $Q$, as well as $P_{\mathbf{r}}$, is continuous. Hence, $Q=P_{\mathbf{r}}$. Since $\mathcal{M}$ is strong finite type, the remaining case is $Q(\mathcal{M})$ finite. Hence, $\mathcal{M}$ is semi-simple. The converse follows from Theorem 4.7.

Proposition 4.9. If $\mathcal{M}$ is a WAP, semi-simple or simple label, then every $\mathcal{N} \in \Theta(\mathcal{M})$ satisfies the corresponding property.

Proof: For $\mathcal{N} \in \Theta(\mathcal{M}), \mathcal{E}(\Theta(\mathcal{M}))$ maps onto $\mathcal{E}(\Theta(\mathcal{N}))$ by restriction. If $\mathcal{E}(\Theta(\mathcal{N}))$ is commutative then $\mathcal{E}(\Theta(\mathcal{N}))$ is and so $\mathcal{N}$ is WAP. The restriction maps $\mathcal{E}_{0}(\Theta(\mathcal{M}))$ onto $\mathcal{E}_{0}(\Theta(\mathcal{N}))$ and so the image of $\mathcal{E}_{*}(\Theta(\mathcal{M}))$ contains $\mathcal{E}_{*}(\Theta(\mathcal{N}))$. Thus, if every $Q \in \mathcal{E}_{*}(\Theta(\mathcal{M}))$ has range in $\mathcal{L} \mathcal{A} \mathcal{B}_{f}$ then the same is true for every element $\mathcal{E}_{*}(\Theta(\mathcal{N}))$. If $\mathcal{E}_{*}(\Theta(\mathcal{M}))$ is empty then the same is true for $\mathcal{E}_{*}(\Theta(\mathcal{N}))$.

We now describe a condition which is easy to check and which implies semisimplicity.

Definition 4.10. A label $\mathcal{M}$ is finitary if it is bounded and whenever $\left\{\mathbf{r}^{i}\right\}$ is a sequence of $\mathbb{N}$-vectors with $\bigcup_{i}$ supp $\mathbf{r}^{i}$ infinite the LIMINF $\left\{\mathcal{M}-\mathbf{r}^{i}\right\}$ is finite. We call this the Finitary Condition.

Clearly, if $\mathcal{M}_{1} \subset \mathcal{M}$ is a label then $\mathcal{M}_{1}$ is finitary if $\mathcal{M}$ is.

For a label $\mathcal{M}$ and $\mathcal{N}$ a nonempty set of $\mathbb{N}$-vectors with $\mathcal{N}$, we define

$$
\mathcal{M}-\mathcal{N}=\{\mathbf{m}: \mathbf{m}+\mathbf{r} \in \mathcal{M} \quad \text { for all } \mathbf{r} \in \mathcal{N}\}=\bigcap_{\mathbf{r} \in \mathcal{N}} \mathcal{M}-\mathbf{r}
$$

The set $\mathcal{M}-\mathcal{N}$ is a label for any nonempty set $\mathcal{N} \subset F I N(\mathbb{N})$, since $\mathcal{M}$ is a label.

Proposition 4.11. Let $\mathcal{M}$ be a bounded label.

(a) The following conditions are equivalent.

(i) If $\left\{S_{i}\right\}$ is a sequence of finite subsets of $\mathbb{N}$ with $\bigcup_{i} S_{i}$ infinite, there are only finitely many subsets $S$ of $\mathbb{N}$ such that eventually $S \cup S_{i} \in S u p p \mathcal{M}$.

(ii) If $\left\{\ell^{i}\right\}$ is a sequence of distinct positive integers then, there are only finitely many $\ell \in \mathbb{N}$ such that eventually $\left\{\ell, \ell^{i}\right\} \in S u p p \mathcal{M}$.

(iii) The label is finitary, i.e. if $\left\{\mathbf{r}^{i}\right\}$ is a sequence of $\mathbb{N}$-vectors with $\bigcup_{i}$ supp $\mathbf{r}^{i}$ infinite then LIMINF $\left\{\mathcal{M}-\mathbf{r}^{i}\right\}$ is finite.

(iv) If $\mathbf{r}^{i}$ is a sequence of $\mathbb{N}$-vectors with $\bigcup_{i}$ supp $\mathbf{r}^{i}$ infinite and $\left\{\mathcal{M}-\mathbf{r}^{i}\right\}$ convergent then then LIM $\left\{\mathcal{M}-\mathbf{r}^{i}\right\}$ is finite.

(v) If $\mathcal{N}$ is infinite then $\mathcal{M}-\mathcal{N}$ is finite, and there is no strictly increasing sequence in the collection $\{\mathcal{M}-\mathcal{N}: \mathcal{N}$ infinite $\}$ of finite labels. 
(vi) If $\left\{\mathbf{r}^{i}: i \in I\right\}$ is a net of $\mathbb{N}$-vectors which is not cofinal constant, and $\left\{\mathcal{M}-\mathbf{r}^{i}: i \in I\right\}$ is convergent then then LIM $\left\{\mathcal{M}-\mathbf{r}^{i}\right\}$ is finite.

(b) If $\mathcal{M}$ is finitary then it is semi-simple.

(c) If $\mathcal{M}$ is finitary, then the following conditions on a finite subset $\mathcal{F}$ of $\mathcal{M}$ are equivalent.

(i) There exists $\mathbf{r}^{i}$ a sequence of $\mathbb{N}$ vectors with $\bigcup_{i}$ supp $\mathbf{r}^{i}$ infinite such that $\operatorname{LIM}\left\{\mathcal{M}-\mathbf{r}^{i}\right\}=\mathcal{F}$.

(ii) There exists $\mathbf{r}^{i}$ a sequence of distinct $\mathbb{N}$-vectors such that LIM $\{\mathcal{M}-$ $\left.\mathbf{r}^{i}\right\}=\mathcal{F}$.

(iii) There is an infinite set $\mathcal{N}$ such that $\mathcal{F}=\mathcal{M}-\mathcal{N}_{1}$ for every infinite subset $\mathcal{N}_{1}$ of $\mathcal{N}$.

Proof: If $\mathcal{N} \subset \mathcal{M}$ then, since $\mathcal{M}$ is bounded, $\mathcal{N}$ is infinite iff $\bigcup \operatorname{Supp} \mathcal{N}$ is infinite. Also, if $\left\{\mathbf{r}^{i}\right\}$ is a sequence of $\mathbb{N}$ vectors with $\bigcup_{i}\left\{\right.$ supp $\left.\mathbf{r}^{i}\right\}$ infinite then we can choose a subsequence of distinct vectors.

(a) (i) $\Rightarrow$ (ii): Let $S_{i}=\left\{\ell^{i}\right\}$.

(ii) $\Rightarrow$ (i): By going to a subsequence we can assume that we can choose $\ell^{i} \in S_{i}$ so that $\left\{\ell^{i}\right\}$ is an infinite sequence of distinct integers. Let $K=\{\ell \in \mathbb{N}$ : eventually $\left.\left\{\ell, \ell^{i}\right\} \in S u p p \mathcal{M}\right\}$. This is a finite set by (ii). If $S \cup S_{i} \in S u p p \mathcal{M}$ then $S \cup\left\{\ell^{i}\right\} \in S u p p \mathcal{M}$ and if $\ell \in S$ then $\left\{\ell, \ell^{i}\right\} \in S u p p \mathcal{M}$. Hence, if eventually $S \cup S_{i} \in S$ upp $\mathcal{M}$ then $S \subset K$. Since the power set of $K$ is finite (i) holds.

(i) $\Rightarrow$ (iii): If $\mathbf{m} \in L I M I N F$ then eventually supp $\mathbf{m} \cup \operatorname{supp} \mathbf{r}^{i} \in S u p p M$. By (i) $\mathcal{M}$ there are only finitely many such sets supp $\mathbf{m}$ and so there are only finitely many $\mathbf{m} \in \mathcal{M}$ with such supports, because $\mathcal{M}$ is bounded.

(iii) $\Leftrightarrow$ (iv): If $\left\{\mathbf{r}^{i}\right\}$ is a sequence with $\bigcup_{i}\left\{\operatorname{supp} \mathbf{r}^{i}\right\}$ infinite then we can choose a subsequence of distinct vectors and then go to a further subsequence $\left\{\mathbf{r}^{i^{\prime}}\right\}$ which is convergent. Assuming (iv) $\operatorname{LIM}\left\{\mathcal{M}-\mathbf{r}^{i^{\prime}}\right\}=\operatorname{LIMINF}\{\mathcal{M}-$ $\left.\mathbf{r}^{i^{\prime}}\right\} \supset \operatorname{LIMINF}\left\{\mathcal{M}-\mathbf{r}^{i}\right\}$ is finite. This shows that (iv) implies (iii). The converse is obvious.

(iii) $\Rightarrow(\mathrm{v})$ : Suppose that $\left\{\mathcal{F}^{i}\right\}$ is a nondecreasing sequence of subsets of $\mathcal{M}$ with each $\mathcal{M}-\mathcal{F}^{i}=\mathcal{N}_{i}$ infinite. So $\mathcal{F}^{i} \subset \mathcal{M}-\mathcal{N}_{i}$. Inductively, choose $\mathbf{r}^{i} \in \mathcal{M}-\mathcal{F}^{i}$ distinct from the $\mathbf{r}^{j}$ 's with $j<i$. Since the sequence $\left\{\mathcal{F}^{i}\right\}$ is a nondecreasing, $\mathcal{F}^{j} \subset \mathcal{M}-\mathbf{r}^{i}$ for $j \leq i$. Hence, $\bigcup_{i} \mathcal{F}^{i} \subset \operatorname{LIMINF}\left\{\mathcal{M}-\mathbf{r}^{i}\right\}$ and so it is finite by (iii). Thus, the sequence $\left\{\mathcal{F}^{i}\right\}$ is eventually constant.

In particular, if $\mathcal{N}$ is infinite and $\mathcal{F}=\mathcal{M}-\mathcal{N}$ then $\mathcal{M}-\mathcal{F} \supset \mathcal{N}$ is infinite and applying the above argument to the sequence which is constantly $\{\mathcal{F}\}$ we see that $\mathcal{F}=\mathcal{M}-\mathcal{N}$ is finite.

$(\mathrm{vi}) \Rightarrow(\mathrm{v})$ : By going to a subsequence we can assume that $\left\{\mathbf{r}^{i}\right\}$ is a sequence of distinct elements. Then $\left\{\bigcap_{j \geq i}\left\{\mathcal{M}-\mathbf{r}^{j}\right\}\right\}$ is a nondecreasing sequence in $\{\mathcal{M}-\mathcal{N}: \mathcal{N}$ infinite $\}$. Hence, its union, which is the limit, is finite by (vi). 
$(\mathrm{vi}) \Rightarrow(\mathrm{iv})$ : Again we can assume that the sequence $\left\{\mathbf{r}^{i}\right\}$ consists of distinct elements. It is then a convergent net which is not cofinal constant. Hence the limit is finite by (vi).

(iii) $\Rightarrow$ (vi): Assume that $\mathcal{N}=\operatorname{LIM}\left\{\mathcal{M}-\mathbf{r}^{i}: i \in I\right\}$ is infinite. Write $\mathcal{N}$ as the union of a strictly increasing sequence of nonempty finite labels $\left\{\mathcal{N}^{k}: k \in \mathbb{N}\right\}$. Since each $\mathcal{N}^{k}$ is finite, eventually $\mathcal{N}^{k} \subset \mathcal{M}-\mathbf{r}^{i}$. Inductively, we can choose $i_{k} \in I$ so that $i_{k} \geq i_{k-1}$ in $I$ and $\mathcal{N}^{k} \subset \mathcal{M}-\mathbf{r}^{i}$ for all $i \geq i_{k}$ in $I$. Furthermore, such the sequence is not cofinal constant, we may choose $i_{k}$ so that $\mathbf{r}^{i_{k}}$ is distinct from $\mathbf{r}^{i_{\ell}}$ for all $\ell<k$ in $\mathbb{N}$. Since, the $\mathcal{N}^{k}$ 's are nonempty, $\left\{\mathbf{r}^{i_{k}}: k \in \mathbb{N}\right\}$ is a sequence of distinct elements of $\mathcal{M}$. Furthermore, $L I M I N F_{k}\left\{\mathcal{M}-\mathbf{r}^{i_{k}}\right\} \supset \bigcup_{k} \mathcal{N}^{k}=\mathcal{N}$. This contradicts (iii). Contrapositively, (iii) implies (vi).

(b) : Let $Q \in \mathcal{E}_{*}(\Theta(\mathcal{M}))$ be the pointwise limit of the net $\left\{P_{\mathbf{r}^{i}}: i \in I\right\}$. Since $Q$ is an external element, the net $\left\{\mathbf{r}^{i}\right\}$ is not cofinal constant. It follows from (v) above that $Q(\mathcal{M})=L I M\left\{\mathcal{M}-\mathbf{r}^{i}\right\}$ is finite.

(c) (i) $\Leftrightarrow$ (ii): This is obvious from our initial remarks.

(ii) $\Rightarrow$ (iii): Assume $\mathbf{r}^{i}$ a sequence of $\mathbb{N}$-vectors with $L I M\left\{\mathcal{M}-\mathbf{r}^{i}\right\}=\mathcal{F}$. By discarding finitely many terms $\mathbf{r}^{i}$ we can assume that the finite set $\mathcal{F}$ equals $\mathcal{M}-\mathcal{N}$ with $\mathcal{N}=\left\{\mathbf{r}^{i}\right\}$. If $\mathcal{N}_{1}$ is any infinite subset of $\mathcal{N}$ then $\mathcal{F}=\mathcal{M}-\mathcal{N} \subset$ $\mathcal{M}-\mathcal{N}_{1}$. On the other hand, if $\mathbf{m} \in \mathcal{M}-\mathcal{N}_{1}$ then $\mathbf{m} \in \mathcal{M}-\mathbf{r}^{i}$ for infinitely many $i$ and so by convergence $\mathbf{m} \in L I M=\mathcal{F}$.

(iii) $\Rightarrow$ (i): If $\mathcal{N}=\left\{\mathbf{r}^{1}, \mathbf{r}^{2}, \ldots\right\}$ is the infinite set given by (iii) then $\left\{\mathbf{r}^{i}\right\}$ is a sequence of distinct elements with $\mathcal{F}=\operatorname{LIM}\left\{\mathcal{M}-\mathbf{r}^{i}\right\}$.

Proposition 4.12. If $\mathcal{M}$ is a label such that $\mathcal{M}-\mathbf{r}$ is a finite label for all $\mathbf{r}>0$, then $\mathcal{M}$ is bounded, simple and finitary.

Proof: If a label $\mathcal{N}$ is unbounded then for some $\ell \in \mathbb{N}, \mathcal{N}$ contains $\mathcal{N}_{1}=$ $\left\{n \chi(\ell): n \in \mathbb{Z}_{+}\right\}$and so $\mathcal{N}-\chi(\ell)$ contains $\mathcal{N}_{1}-\chi(\ell)=\mathcal{N}_{1}$, which is infinite. Hence, $\mathcal{M}$ is bounded..

If $\left\{\mathbf{r}^{i}\right\}$ is a sequence in $\mathcal{M}$ with $\bigcup_{i} \operatorname{supp}\left(\mathbf{r}^{i}\right)$ infinite then for any $\mathbf{r}>0 \mathcal{M}-\mathbf{r}$ is finite and so $\bigcup_{i}\left\{\operatorname{supp}\left(\mathbf{r}^{i}\right): \mathbf{r}^{i} \in \mathcal{M}-\mathbf{r}\right\}$ is finite. So it cannot be true that eventually $\mathbf{r} \in \mathcal{M}-\mathbf{r}^{i}$. Hence, $\operatorname{LIMINF}\left\{\mathcal{M}-\mathbf{r}^{i}\right\}=0$. It follows that $\mathcal{M}$ is finitary. So $\left\{\mathcal{M}-\mathbf{r}^{i}\right\}$ is convergent with limit 0 . Hence, $\mathcal{M}$ is bounded and semi-simple and so is of finite type.

If $\bigcup_{i} \operatorname{supp}\left(\mathbf{r}^{i}\right)$ is finite then the sequence is cofinal constant by Lemma 4.6 (b). So if the sequence $\left\{\mathcal{M}-\mathbf{r}^{i}\right\}$ is convergent then its limit is $\mathcal{M}-\mathbf{r}$ for some r.

Finally, if $\left\{\mathcal{M}-\mathbf{r}^{i}\right\}$ is convergent and infinitely often $\mathbf{r}^{i} \notin \mathcal{M}$ then the limit is $\emptyset$. 
Since $\mathcal{M}$ is bounded, $\emptyset=\mathcal{M}-\mathbf{r}$ for and if $\mathcal{M}$ is nonempty then since $\mathcal{M}$ is of finite type, $\max \mathcal{M}$ is nonempty. If $\mathbf{r} \in \max \mathcal{M}$ then $0=\mathcal{M}-\mathbf{r}$. It follows $\Theta(\mathcal{M})=\{\mathcal{M}-\mathbf{r}: \mathbf{r} \in F I N(\mathbb{N})\}$ and so $\mathcal{M}$ is simple.

\subsection{WAP subshifts.}

We now apply the above results to the associated subshifts. We will repeatedly use the following fundamental fact concerning the subshift $(X(\mathcal{M}), S)$ associated with a label $\mathcal{M}$ of finite type.

Theorem 4.13. Let $\mathcal{M}$ be a label of finite type.

(a) If $z \in X(\mathcal{M})$ with $z \neq e=x[\emptyset]$, then there exists a unique pair $(t, \mathcal{N}) \in$ $\mathbb{Z} \times \Theta(\mathcal{M})$ such that $z=S^{t}(x[\mathcal{N}])$ and for every $(t, \mathcal{N}) \in \mathbb{Z} \times \Theta(\mathcal{M})$, $S^{t}(x[\mathcal{N}]) \in X(\mathcal{M})$.

(b) If $w \in X_{+}(\mathcal{M})$ with $z \neq e=x[\emptyset]$, then there exists a unique pair $(t, \mathcal{N}) \in \mathbb{Z} \times \Theta(\mathcal{M})$ such that $z=S^{t}\left(x_{+}[\mathcal{N}]\right)$ and for every $(t, \mathcal{N}) \in$ $\mathbb{Z} \times \Theta(\mathcal{M}), S^{t}\left(x_{+}[\mathcal{N}]\right) \in X_{+}(\mathcal{M})$.

Proof: By Theorem $3.34 X(\mathcal{M})=\left\{S^{t}(x[\mathcal{N}]):(t, \mathcal{N}) \in \mathbb{Z} \times \Theta(\mathcal{M})\right\}$ and similarly for $X_{+}(\mathcal{M})$. If $S^{t_{1}}\left(x\left[\mathcal{N}_{1}\right]\right)=S^{t_{2}}\left(x\left[\mathcal{N}_{2}\right]\right)$ with $\mathcal{N}_{2} \neq \emptyset$ then $S^{t_{1}-t_{2}}\left(x\left[\mathcal{N}_{1}\right]\right)=$ $x\left[\mathcal{N}_{2}\right]$, which is symmetric about 0 and has a 1 at position 0 . Hence $\mathcal{N}_{1} \neq \emptyset$ and $t_{1}-t_{2}=0$. Finally, $\mathcal{N}_{1}=\mathcal{N}_{2}$ by Theorem 3.18. Similarly, if $S^{t_{1}-t_{2}}\left(x_{+}\left[\mathcal{N}_{1}\right]\right)=$ $x_{+}\left[\mathcal{N}_{2}\right]$ then since the latter has a 1 at position 0 and 0 's at all negative positions it follows that $t_{1}-t_{2}=0$ and again $\mathcal{N}_{1}=\mathcal{N}_{2}$ by Theorem 3.18.

We now describe the enveloping semigroup version of this result. Recall that for $\mathcal{M} \neq F I N(\mathbb{N})$, and $\mathbf{r} \notin \mathcal{M}, P_{\mathbf{r}}=U \in \mathcal{E}(\Theta(\mathcal{M}))$ is the idempotent with $U(\mathcal{N})=\emptyset$ for all $\mathcal{N} \in \Theta(\mathcal{M})$

We use the continuous, surjective homomorphisms $D: E_{b}(X(\mathcal{M}), S) \rightarrow$ $\mathcal{E}(\Theta(\mathcal{M})), D_{+}: E_{b}\left(X_{+}(\mathcal{M}), S\right) \rightarrow \mathcal{E}(\Theta(\mathcal{M}))$ from $(3.34)$.

Lemma 4.14. Assume $\mathcal{M}$ is a label of finite type.

(a) If $q_{1}, q_{2} \in E(X(\mathcal{M}), S)$ with $q_{1}(x[\mathcal{N}])=q_{2}(x[\mathcal{N}])$ for every $\mathcal{N} \in \Theta(\mathcal{M})$ then $q_{1}=q_{2}$. If $q_{1}, q_{2} \in E\left(X_{+}(\mathcal{M}), S\right)$ with $q_{1}\left(x_{+}[\mathcal{N}]\right)=q_{2}\left(x_{+}[\mathcal{N}]\right)$ for every $\mathcal{N} \in \Theta(\mathcal{M})$ then $q_{1}=q_{2}$.

(b) The maps $D$ and $D_{+}$are injective and so are homeomorphisms and monoid isomorphisms. 
Proof: (a) If $q_{1}, q_{2} \in E(X(\mathcal{M}), S)$ with $q_{1}=q_{2}$ on $\{x[\mathcal{N}]: \mathcal{N} \in \Theta(\mathcal{M})\}$ then since $q_{1}$ and $q_{2}$ commute with all of the $S^{t}$ s, it follows from Theorem 4.13 that $q_{1}=q_{2}$ on all of $X(\mathcal{M})$.

(b) If $Q=D\left(q_{1}\right)=D\left(q_{2}\right)$ then $q_{1}(x[\mathcal{N}])=x[Q(\mathcal{N})]=q_{2}(x[\mathcal{N}])$ for all $\mathcal{N} \in$ $\Theta(\mathcal{M})$. By $\left(\right.$ a) $q_{1}=q_{2}$. Thus, $D$ is bijective and so is a monoid isomorphism and is a homeomorphism by compactness.

The proofs for $X_{+}(\mathcal{M})$ are the same.

We will denote the inverse isomorphisms as

$$
\bar{D}: \mathcal{E}(\Theta(\mathcal{M})) \rightarrow E_{b}(X(\mathcal{M}), S) \quad \text { and } \quad \bar{D}_{+}: \mathcal{E}(\Theta(\mathcal{M})) \rightarrow E_{b}\left(X_{+}(\mathcal{M}), S\right)
$$

Theorem 4.15. Let $\mathcal{M}$ be a label of finite type.

(a) For every $Q \in \mathcal{E}(\Theta(\mathcal{M})), \bar{D}(Q) \in E(X(\mathcal{M}), S)$ and $\bar{D}_{+}(Q) \in E\left(X_{+}(\mathcal{M}), S\right)$ are the unique elements such that

$$
\bar{D}(Q)(x[\mathcal{N}])=x[Q(\mathcal{N})], \quad \bar{D}_{+}(Q)\left(x_{+}[\mathcal{N}]\right)=x_{+}[Q(\mathcal{N})] \quad \text { for all } \quad \mathcal{N} \in \Theta(\mathcal{M})
$$

with $\bar{D}(U)$, (and $\left.\bar{D}_{+}(U)\right)$ the idempotent u mapping $X(\mathcal{M})\left(\right.$ resp. $X_{+}(\mathcal{M})$ ) to the fixed point $e=x[\emptyset]=x_{+}[\emptyset]$.

(b) For every $q \in E(X(\mathcal{M}), S)$ with $q \neq u$ there exists a unique pair $(t, Q) \in$ $\mathbb{Z} \times \mathcal{E}\left(\Theta(\mathcal{M})\right.$ ) such that $q=S^{t} \bar{D}(Q)$. For every $q \in E\left(X_{+}(\mathcal{M}), S\right)$ with $q \neq u$ there exists a unique pair $(t, Q) \in \mathbb{Z} \times \mathcal{E}(\Theta(\mathcal{M}))$ such that $q=S^{t} \bar{D}_{+}(Q)$.

(c) For every $\mathbf{r} \in F I N(\mathbb{N}), p_{\mathbf{r}}=\bar{D}\left(P_{\mathbf{r}}\right) \in E(X(\mathcal{M}), S)$ and $p_{\mathbf{r}}^{+}=\bar{D}_{+}\left(P_{\mathbf{r}}\right) \in$ $E\left(X_{+}(\mathcal{M}), S\right)$ are continuous so that $(\mathbf{r}, z) \mapsto p_{\mathbf{r}}(z)$ and $(\mathbf{r}, z) \mapsto p_{\mathbf{r}}^{+}(z)$ define continuous semigroup actions $F I N(\mathbb{N}) \times X(\mathcal{M}) \rightarrow X(\mathcal{M})$ and $F I N(\mathbb{N}) \times X_{+}(\mathcal{M}) \rightarrow X_{+}(\mathcal{M})$ respectively

Proof: We will give the proofs just for the $(X(\mathcal{M}), S)$ case as the $\left(X_{+}(\mathcal{M}), S\right)$ arguments are completely analogous.

(a): This is a restatement of Lemma 4.14.

(b): For $q \in E(X(\mathcal{M}), S)$ assume that $\left\{t^{i}\right\}$ is a net in $\mathbb{Z}$ so that $\left\{S^{t^{i}}\right\}$ converges pointwise to $q$.

If $q(x[\mathcal{M}])=e$ then by Lemma $3.24 \xi_{\mathcal{M}}\left(t^{i}\right) \rightarrow \infty$ and so $\xi_{\mathcal{N}}\left(t^{i}\right) \rightarrow \infty$ for any label $\mathcal{N}$ contained in $\mathcal{M}$. Hence, $q(x[\mathcal{N}])=e$ for all $\mathcal{N} \in \Theta(\mathcal{M})$ and so from Theorem 4.13 it follows that $q(z)=e$ for all $z \in X(\mathcal{M})$, i.e. $q=u=\bar{D}(U)$.

If $q(x[\mathcal{M}])_{a}=1$ for some $a \in Z$ we may replace $q$ by $S^{-a} q$ and so assume that $q(x[\mathcal{M}])_{0}=1$. This implies that eventually $t^{i} \in A[\mathcal{M}]$. Since $\mathcal{M}$ is of finite type, Lemma 3.21(b) implies that there exists $b \in \mathbb{Z}$ so that $t^{i}-b$ is eventually in $A[\mathcal{N}]$ with $j_{r}\left(t^{i}-b\right) \rightarrow \infty$. Thus, we may assume that $t^{i}=k+s^{i}$ with $s^{i} \in A[\mathcal{N}]$ and $j_{r}\left(s^{i}\right) \rightarrow \infty$. Let $\mathbf{r}^{i}=\mathbf{r}\left(s^{i}\right)$. By going to a subnet we 
may assume that $\left\{P_{\mathbf{r}^{i}}\right\}$ converges to some $Q \in \mathcal{E}(\Theta(\mathcal{M}))$. From the Coherence Lemma again, it follows that $S^{-k} q=\operatorname{Lim}\left\{S^{s^{i}}\right\}=\bar{D}(Q)$.

Finally, if $S^{t_{1}} \bar{D}\left(Q_{1}\right)=q=S^{t_{2}} \bar{D}\left(Q_{2}\right)$, then

$$
S^{t_{1}-t_{2}}\left(x\left[Q_{1}(\mathcal{M})\right]\right)=S^{t_{1}-t_{2}} \bar{D}\left(Q_{1}\right)(x[\mathcal{M}])=\bar{D}\left(Q_{2}\right)(x[\mathcal{M}])=x\left[Q_{2}(\mathcal{M})\right] .
$$

Since $Q_{2}(\mathcal{M}) \neq \emptyset$ it follows from the uniqueness in Theorem 4.13 that $t_{1}-t_{2}=$ 0 . Hence, $\bar{D}\left(Q_{1}\right)=\bar{D}\left(Q_{2}\right)$ and so $Q_{1}=Q_{2}$ because $\bar{D}$ is an isomorphism. It follows that the representation is unique.

(c): Since $P_{\mathbf{r}}$ commutes with every $Q \in \mathcal{E}(\Theta(\mathcal{M}))$ it follows from (b) that $p_{\mathbf{r}}$ commutes with every $q \in E(X(\mathcal{M}), S)$ and so $p_{\mathbf{r}}$ is continuous by Proposition 1.8 .

Since $\bar{D}$ is a semigroup isomorphism and $P_{\mathbf{r}_{1}} P_{\mathbf{r}_{2}}=P_{\mathbf{r}_{1}+\mathbf{r}_{2}}$, it follows that $p_{\mathbf{r}_{1}} p_{\mathbf{r}_{2}}=p_{\mathbf{r}_{1}+\mathbf{r}_{2}}$ and so $(\mathbf{r}, z) \mapsto p_{\mathbf{r}}(z)$ defines a continuous semigroup action.

Remark 4.16. From the representation in (b) it is clear that $\bar{D}$ maps $\mathcal{A}(\Theta(\mathcal{M}))$ into $A(X(\mathcal{M}), S)$.

Let $(\mathbb{Z} \times \mathcal{E}(\Theta(\mathcal{M})) /(\mathbb{Z} \times\{U\})$ denote the quotient of the product semigroup obtained by identifying all of the points of $\mathbb{Z} \times\{U\}$ with the single point $U$. Define

$$
\begin{aligned}
\hat{D}:\left(\mathbb{Z} \times \mathcal{E}(\Theta(\mathcal{M})) /(\mathbb{Z} \times\{U\}) \rightarrow E(X(\mathcal{M}), S) \quad \text { by } \quad(t, Q) \mapsto S^{t} \bar{D}(Q),\right. \\
\hat{D}_{+}:\left(\mathbb{Z} \times \mathcal{E}(\Theta(\mathcal{M})) /(\mathbb{Z} \times\{U\}) \rightarrow E\left(X_{+}(\mathcal{M}), S\right) \quad \text { by } \quad(t, Q) \mapsto S^{t} \bar{D}_{+}(Q) .\right.
\end{aligned}
$$

From Theorem 4.15 we immediately obtain:

Corollary 4.17. The mappings $\hat{D}$ and $\hat{D}^{+}$are semigroup isomorphisms.

N.B. This is an algebraic result with no topological implications.

The maximum label $F I N(\mathbb{N})$ is simple since $\Theta(F I N(\mathbb{N}))=\{F I N(\mathbb{N})\}$. Hence, it is an unbounded WAP label. Since it is recurrent, $X(F I N(\mathbb{N}))$ is uncountable and so $(X(F I N(\mathbb{N})), S)$ is not WAP. In fact, it is even not tame; see Proposition 5.8 below.

Lemma 4.18. If a bounded label is WAP then it is of finite type.

Proof: If $\mathcal{M}$ is not of finite type then $\Theta(\mathcal{M})$ contains a recurrent label by Corollary 2.25. Since a subsystem of a WAP system is WAP, it suffices to show that a non-trivial bounded, recurrent label $\mathcal{M}$ is not WAP. 
Since $\mathcal{M}$ is recurrent, there exists a sequence $\left\{\mathbf{r}^{i}>0\right\}$ such that $P_{\mathbf{r}^{i}}(\mathcal{M}) \rightarrow$ $\mathcal{M}$. Choose a subnet $\left\{\mathbf{r}^{i^{\prime}}\right\}$ so that $P_{\mathbf{r}^{i^{\prime}}} \rightarrow Q$ in $\mathcal{E}(\Theta(\mathcal{M}))$. Clearly, $Q(\mathcal{M})=$ $\mathcal{M}=P_{\mathbf{0}}(\mathcal{M})$. If $Q$ were continuous then since $\mathcal{M}$ is a transitive point for $\Theta(\mathcal{M})$ we would have $Q=P_{\mathbf{0}}=i d$ on $\Theta(\mathcal{M})$. Because $\mathcal{M}$ is bounded, $0 \in \Theta(\mathcal{M})$ by Lemma 2.18. But $0-\mathbf{r}^{i}=\emptyset$ for all $i$. Hence, $Q(0)=\emptyset \neq 0=P_{\mathbf{0}}(0)$.

So we obtain

Theorem 4.19. For a label $\mathcal{M}$ the following are equivalent.

(a) The label $\mathcal{M}$ is bounded and WAP.

(b) The cascade $(X(\mathcal{M}), S)$ is WAP.

(c) The cascade $\left(X_{+}(\mathcal{M}), S\right)$ is WAP.

In that case, $\Theta(\mathcal{M})$ and $X(\mathcal{M})$ are countable and $\mathcal{M}$ is of finite type.

Proof: If $\mathcal{M}$ is bounded and WAP then by Lemma 4.18 it is of finite type and $\mathcal{E}(\Theta(\mathcal{M}))$ is commutative. Hence, $(\mathbb{Z} \times \mathcal{E}(\Theta(\mathcal{M})) /(\mathbb{Z} \times\{U\})$ is commutative and so from Corollary 4.17 it follows that $E(X(\mathcal{M}), S)$ and $E\left(X_{+}(\mathcal{M}), S\right)$ are commutative. As these are topologically transitive cascades, they are WAP.

If either $(X(\mathcal{M}), S)$ and $\left(X_{+}(\mathcal{M}), S\right)$ is WAP then as WAP subshifts the spaces are countable. Since $\Theta(\mathcal{M})$ injects into $X(\mathcal{M})$ and $X_{+}(\mathcal{M})$, it is is countable and so $\mathcal{M}$ is of finite type. From Corollary 4.17 again it follows that $\mathcal{E}(\Theta(\mathcal{M}))$ is commutative and so $\mathcal{M}$ is a WAP label.

In summary, we have the implications:

$$
\begin{aligned}
& \text { simple } \Longrightarrow \text { semi-simple } \Longrightarrow \text { WAP, } \\
& \text { finitary } \Longrightarrow \text { bounded and semi-simple } \Longrightarrow \\
& \text { strong finite type } \Longrightarrow \text { finite type, } \\
& \text { bounded and WAP } \Longrightarrow \text { finite type. }
\end{aligned}
$$

\subsection{Constructions and examples.}

We turn now to the tools we will use to construct many examples of WAP labels.

Recall that for labels $\mathcal{M}_{1}$ and $\mathcal{M}_{2}$ we defined

$$
\mathcal{M}_{1} \oplus \mathcal{M}_{2}=\left\{\mathbf{m}_{1}+\mathbf{m}_{2}: \mathbf{m}_{1} \in \mathcal{M}_{1}, \mathbf{m}_{2} \in \mathcal{M}_{2}\right\} .
$$

Recall that $\left(\mathcal{M}_{1}, \mathcal{M}_{2}\right) \mapsto \mathcal{M}_{1} \oplus \mathcal{M}_{2}$ is a continuous map from $\mathcal{L} \mathcal{A B} \times \mathcal{L} \mathcal{A B} \rightarrow$ $\mathcal{L} \mathcal{A B}$. So if $\Phi_{1}, \Phi_{2} \subset \mathcal{L} \mathcal{A B}$ are compact then $\Phi_{1} \oplus \Phi_{2}$ is compact and is 
therefore closed. If either $\mathcal{M}_{1}$ or $\mathcal{M}_{2}$ is empty then $\mathcal{M}_{1} \oplus \mathcal{M}_{2}=\emptyset$. Otherwise, $\mathcal{M}_{1} \oplus \mathcal{M}_{2} \neq \emptyset$.

Definition 4.20. Let $M$ be a collection of finite subsets of $\mathbb{N}$. Call it hereditary if $A \subset B$ and $B \in M$ implies $A \in M$. We say that $M$ f-contains $L \subset \mathbb{N}$ if every finite subset of $L$ is in $M$, i.e. $\mathcal{P}_{f} L \subset M$.

We will need a combinatorial lemma.

For two hereditary collections $M_{1}, M_{2}$ define $M_{1} \oplus M_{2}=\left\{A_{1} \cup A_{2}: A_{1} \in\right.$ $\left.M_{1}, A_{2} \in M_{2}\right\}$.

Lemma 4.21. Let $M_{1}$ and $M_{2}$ be hereditary collections of finite subsets of $\mathbb{N}$. If $M_{1} \oplus M_{2} f$-contains an infinite set then either $M_{1}$ or $M_{2} f$-contains an infinite set.

Proof: Let $L=\left\{\ell_{1}, \ell_{2}, \ldots\right\}$ be a counting for an infinite set f-contained in $M_{1} \oplus M_{2}$. Define the directed binary tree with vertices at level $n=0,1, \ldots$ consisting of the $2^{n}$ ordered partitions $(A, B)$ of $\left\{\ell_{1}, \ldots, \ell_{n}\right\}$. Connect $(A, B)$ to the $n+1$ level vertices $\left(A \cup\left\{\ell_{n+1}\right\}, B\right)$ and $\left(A, B \cup\left\{\ell_{n+1}\right\}\right)$. The set of paths to infinity forms a Cantor set. Call a path good at level $n$ if for the partition $\left(A_{n}, B_{n}\right)$ at level $n, A_{n} \in M_{1}$ and $B_{n} \in M_{2}$. Since $M_{1} \oplus M_{2}$ fcontains $L$, the set $G_{n}$ of paths good at level $n$ is nonempty. Each $G_{n}$ is closed and $G_{n+1} \subset G_{n}$. So the intersection contains a path $\left\{\left(A^{i}, B^{i}\right): i=0,1, \ldots\right\}$. Let $A^{\infty}=\bigcup A^{i}, B^{\infty}=\bigcup B^{i}$. Clearly, $\left\{A^{i}\right\}$ is a nondecreasing sequence of finite sets in $M_{1}$ with union $A^{\infty}$ and so $A^{\infty}$ is f-contained in $M_{1}$. Similarly, $B^{\infty}$ is f-contained in $M_{2}$. Since $A^{\infty} \cup B^{\infty}=L$, at least one of them is infinite.

For a label $\mathcal{M}$, if $\mathbf{m} \in \mathcal{M}$ then $\mathbf{0} \leq \chi(\operatorname{supp} \mathbf{m}) \leq \mathbf{m}$ and so $\chi(\operatorname{supp} \mathbf{m}) \in \mathcal{M}$. Hence, Supp $\mathcal{M}=\{A \subset \mathbb{N}: \chi(A) \in \mathcal{M}\}$. Thus, Supp $\mathcal{M}$ is a hereditary collection of finite subsets of $\mathbb{N}$ with $\operatorname{Supp} \mathcal{M}=\emptyset$ iff $\mathcal{M}=\emptyset$. Thus, Proposition 2.5(b) says that a bounded label $\mathcal{M}$ is not of finite type iff $S$ upp $\mathcal{M}$ f-contains some infinite subset.

Proposition 4.22. Let $\mathcal{M}_{1}, \mathcal{M}_{2}$ be bounded, nonempty labels.

The label $\mathcal{M}_{1} \oplus \mathcal{M}_{2}$ is nonempty and bounded. It is of finite type iff $\mathcal{M}_{1}$ and $\mathcal{M}_{2}$ are labels of finite type.

If $\mathcal{M}_{1}$ is finite and $\mathcal{M}_{2}$ is finitary, then $\mathcal{M}_{1} \oplus \mathcal{M}_{2}$ is finitary.

Proof: Clearly, $\rho\left(\mathcal{M}_{1} \oplus \mathcal{M}_{2}\right)=\rho\left(\mathcal{M}_{1}\right)+\rho\left(\mathcal{M}_{2}\right)$. So $\mathcal{M}_{1} \oplus \mathcal{M}_{2}$ is bounded and nonempty if $\mathcal{M}_{1}$ and $\mathcal{M}_{2}$ are.

Now assume that $\mathcal{M}_{1}$ and $\mathcal{M}_{2}$ are nonempty labels of finite type. If $\mathcal{M}_{1} \oplus \mathcal{M}_{2}$ is not of finite type then $\operatorname{Supp}(\mathcal{N} \oplus \mathcal{M})$ f-contains an infinite set by Proposition $2.5(\mathrm{c})$. 
Since

$$
\operatorname{Supp}\left(\mathcal{M}_{1} \oplus \mathcal{M}_{2}\right)=\left(\operatorname{Supp} \mathcal{M}_{1}\right) \oplus\left(\operatorname{Supp} \mathcal{M}_{2}\right)
$$

it follows from Lemma 4.21 that either Supp $\mathcal{M}_{1}$ or Supp $\mathcal{M}_{2}$ f-contains an infinite set. Thus, $\mathcal{M}_{1}$ and $\mathcal{M}_{2}$ of finite type implies $\mathcal{M}_{1} \oplus \mathcal{M}_{2}$ is of finite type. The converse is obvious since $\mathcal{M}_{1} \cup \mathcal{M}_{2} \subset \mathcal{M}_{1} \oplus \mathcal{M}_{2}$.

Now assume that $\mathcal{M}_{1}$ is finite and $\mathcal{M}_{2}$ is finitary. To show that $\mathcal{M}_{1} \oplus \mathcal{M}_{2}$ is finitary, we apply condition (ii) of Proposition 4.11 (a). Let $\left\{\ell^{i}\right\}$ be a sequence of distinct positive integers. Since $\mathcal{M}_{1}$ is finite, $K=\bigcup S u p p \mathcal{M}_{1}$ is finite and we may assume, by discarding finitely many elements that $\ell^{i} \notin K$ for all $i$. Since $K$ is finite, it suffices to show that $\{\ell \in \mathbb{N} \backslash K$ : such that eventually $\left.\left\{\ell, \ell^{i}\right\} \in \operatorname{Supp} \mathcal{M}_{1} \oplus \mathcal{M}_{2}\right\}$ is finite. Since $\ell, \ell^{i} \notin K$,

$$
\begin{aligned}
\left\{\ell, \ell^{i}\right\} \in \operatorname{Supp} \mathcal{M}_{1} \oplus \mathcal{M}_{2} & \left.\Longleftrightarrow \chi(\ell)+\chi\left(\ell^{i}\right) \in \mathcal{M}_{1} \oplus \mathcal{M}_{2}\right\} \\
\Longleftrightarrow \chi(\ell)+\chi\left(\ell^{i}\right) \in \mathcal{M}_{2} & \Longleftrightarrow\left\{\ell, \ell^{i}\right\} \in \operatorname{Supp} \mathcal{M}_{2} .
\end{aligned}
$$

Since $\mathcal{M}_{2}$ is finitary, the set of $\ell$ such that eventually $\left\{\ell, \ell^{i}\right\} \in \operatorname{Supp} \mathcal{M}_{2}$ is finite.

Remark 4.23. If $\mathcal{M}_{1} \oplus \mathcal{M}_{2}$ is finitary and $\mathcal{M}_{2}$ is infinite then $\mathcal{M}_{1}$ must be finite since if $\left\{\mathbf{r}^{i}\right\}$ is an infinite sequence of distinct positive vectors in $\mathcal{M}_{2}$ then $\mathcal{M}_{1} \subset \operatorname{LIMINF}\left\{\left(\mathcal{M}_{1} \oplus \mathcal{M}_{2}\right)-\mathbf{r}^{i}\right\}$.

A pointed space $(X, *)$ is a compact space with a chosen base point. If $\left(X_{1}, *_{1}\right)$ and $\left(X_{2}, *_{2}\right)$ are pointed spaces then the wedge is $X_{1} \vee X_{2}=\left(X_{1} \times\right.$ $\left.\left\{*_{2}\right\} \cup\left\{*_{1}\right\} \times X_{2}\right)$ with base point $\left(*_{1}, *_{2}\right)$ and the smash product is the quotient space $X_{1} \wedge X_{2}=\left(X_{1} \times X_{2}\right) /\left(X_{1} \vee X_{2}\right)$, i.e. with the wedge identified to a point, the base point of the smash. If $A_{1} \subset X_{1}, A_{2} \subset X_{2}$, we let $A_{1} \wedge A_{2}$ denote the image of $A_{1} \times A_{2}$ in the smash product.

If $\mathcal{M}$ is a label other than $F I N(\mathbb{N})$ we use $\emptyset$ as the base point for $[[\mathcal{M}]]$, the closed set of labels contained in $\mathcal{M}$, and for $\Theta(\mathcal{M})$. We use the retraction $U$ to $\emptyset$ as the base point for the enveloping semigroup $\mathcal{E}(\Theta(\mathcal{M}))$. Since $\{U\}$ is an ideal in $\mathcal{E}(\Theta(\mathcal{M}))$, it follows that for $\mathcal{M}_{1}, \mathcal{M}_{2} \neq F I N(\mathbb{N}), \mathcal{E}\left(\Theta\left(\mathcal{M}_{1}\right)\right) \vee \mathcal{E}\left(\Theta\left(\mathcal{M}_{2}\right)\right)$ is an ideal in the product monoid $\mathcal{E}\left(\Theta\left(\mathcal{M}_{1}\right)\right) \times \mathcal{E}\left(\Theta\left(\mathcal{M}_{2}\right)\right)$ and the quotient map $\mathcal{E}\left(\Theta\left(\mathcal{M}_{1}\right)\right) \times \mathcal{E}\left(\Theta\left(\mathcal{M}_{2}\right)\right) \rightarrow \mathcal{E}\left(\Theta\left(\mathcal{M}_{1}\right)\right) \wedge \mathcal{E}\left(\Theta\left(\mathcal{M}_{2}\right)\right)$ induces a monoid structure on the smash so that the quotient map is a monoid homomorphism.

Definition 4.24. For nonempty labels $\mathcal{M}_{1}, \mathcal{M}_{2}$ we have $\left(\bigcup \operatorname{Supp} \mathcal{M}_{1}\right) \cap$ $\left(\bigcup\right.$ Supp $\left.\mathcal{M}_{2}\right)=\emptyset$ iff $\mathcal{M}_{1} \cap \mathcal{M}_{2}=0=\{\mathbf{0}\}$. In that case we will say that $\mathcal{M}_{1}$ and $\mathcal{M}_{2}$ are disjoint labels. 
Assume $\mathcal{M}_{1}$ and $\mathcal{M}_{2}$ are disjoint, positive labels, i.e. neither is empty or 0 . If $\mathbf{r} \in \mathcal{M}_{1} \oplus \mathcal{M}_{2}$ then $\mathbf{r}=\mathbf{r}_{1}+\mathbf{r}_{2}$ with $\mathbf{r}_{1} \in \mathcal{M}_{1}$ and $\mathbf{r}_{2} \in \mathcal{M}_{2}$ uniquely determined by $\mathbf{r}$. For example, $\mathbf{r} \in \mathcal{M}_{1}$ iff $\mathbf{r}_{2}=0$. If $\mathbf{r} \in \mathcal{M}_{1} \oplus \mathcal{M}_{2}$, then $\left(\mathcal{M}_{1} \oplus \mathcal{M}_{2}\right)-\mathbf{r}=\left(\mathcal{M}_{1}-\mathbf{r}_{1}\right) \oplus\left(\mathcal{M}_{2}-\mathbf{r}_{2}\right)$. Assume $\mathcal{N}_{1} \in\left[\left[\mathcal{M}_{1}\right]\right]$ and $\mathcal{N}_{2} \in\left[\left[\mathcal{M}_{2}\right]\right]$ with neither $\mathcal{N}_{1}$ nor $\mathcal{N}_{2}$ empty then $\mathbf{r} \in \mathcal{N}_{1} \oplus \mathcal{N}_{2}$ iff $\mathbf{r}_{1} \in \mathcal{N}_{1}$ and $\mathbf{r}_{2} \in \mathcal{N}_{2}$. It follows that the restriction

$$
\oplus:\left(\left[\left[\mathcal{M}_{1}\right]\right] \backslash\{\emptyset\}\right) \times\left(\left[\left[\mathcal{M}_{2}\right]\right] \backslash\{\emptyset\}\right) \rightarrow\left[\left[\mathcal{M}_{1} \oplus \mathcal{M}_{2}\right]\right] \backslash\{\emptyset\}
$$

is injective. Since $\mathcal{N}_{1} \oplus \mathcal{N}_{2}=\emptyset$ iff $\mathcal{N}_{1}$ or $\mathcal{N}_{2}$ is empty, we see that there is an induced injection between compact spaces:

$$
\widehat{\oplus}:\left[\left[\mathcal{M}_{1}\right]\right] \wedge\left[\left[\mathcal{M}_{2}\right]\right] \rightarrow\left[\left[\mathcal{M}_{1} \oplus \mathcal{M}_{2}\right]\right] .
$$

Theorem 4.25. Assume $\mathcal{M}_{1}$ and $\mathcal{M}_{2}$ are positive disjoint labels with $\mathcal{M}_{1} \oplus$ $\mathcal{M}_{2} \neq F I N(\mathbb{N})$. The map $\widehat{\oplus}$ restricts to a homeomorphism

$$
\widehat{\oplus}: \Theta\left(\mathcal{M}_{1}\right) \wedge \Theta\left(\mathcal{M}_{2}\right) \rightarrow \Theta\left(\mathcal{M}_{1} \oplus \mathcal{M}_{2}\right) .
$$

For $Q_{1} \in \mathcal{E}\left(\Theta\left(\mathcal{M}_{1}\right)\right), Q_{2} \in \mathcal{E}\left(\Theta\left(\mathcal{M}_{2}\right)\right), Q_{1} \widehat{\oplus} Q_{2}$ is well-defined by $\left(Q_{1} \widehat{\oplus} Q_{2}\right)\left(\mathcal{N}_{1} \oplus\right.$ $\left.\mathcal{N}_{2}\right)=\left(Q_{1}\left(\mathcal{N}_{1}\right)\right) \oplus\left(Q_{2}\left(\mathcal{N}_{2}\right)\right)$. This defines a monoid isomorphism

$$
\widehat{\oplus}: \mathcal{E}\left(\Theta\left(\mathcal{M}_{1}\right)\right) \wedge \mathcal{E}\left(\Theta\left(\mathcal{M}_{2}\right)\right) \rightarrow \mathcal{E}\left(\Theta\left(\mathcal{M}_{1} \oplus \mathcal{M}_{2}\right)\right) \text {. }
$$

Proof: Since the labels are positive and disjoint, neither equals $F I N(\mathbb{N})$.

As described above, $\mathbf{r}=\mathbf{r}_{1}+\mathbf{r}_{2}$ with $\mathbf{r}_{1} \in \mathcal{M}_{1}, \mathbf{r}_{2} \in \mathcal{M}_{2}$ implies $P_{\mathbf{r}}(\mathcal{M})=$ $P_{\mathbf{r}_{1}}\left(\mathcal{M}_{1}\right) \oplus P_{\mathbf{r}_{2}}\left(\mathcal{M}_{2}\right)$. Since $\Theta\left(\mathcal{M}_{1} \oplus \mathcal{M}_{2}\right)$ is closed it follows that $\widehat{\oplus}$ maps $\Theta\left(\mathcal{M}_{1}\right) \wedge$ $\Theta\left(\mathcal{M}_{2}\right)$ into $\Theta\left(\mathcal{M}_{1} \oplus \mathcal{M}_{2}\right)$.

Now let $\left\{Q_{1}^{i}: i \in I\right\}$ and $\left\{Q_{2}^{j}: j \in J\right\}$ be nets in $\mathcal{E}\left(\Theta\left(\mathcal{M}_{1}\right)\right)$ and $\mathcal{E}\left(\Theta\left(\mathcal{M}_{2}\right)\right)$, respectively, converging to $Q_{1}$ and $Q_{2}$. By continuity of $\oplus$, for any $\mathcal{N}_{1} \in$ $\Theta\left(\mathcal{M}_{1}\right), \mathcal{N}_{2} \in \Theta\left(\mathcal{M}_{2}\right)$ the net $\left\{Q_{1}^{i}\left(\mathcal{N}_{1}\right) \oplus Q_{2}^{j}\left(\mathcal{N}_{j}\right)(i, j) \in I \times J\right\}$ converges to $Q_{1}\left(\mathcal{N}_{1}\right) \oplus Q_{2}\left(\mathcal{N}_{2}\right)$, which is empty iff either $Q_{1}\left(\mathcal{N}_{1}\right)$ or $Q_{2}\left(\mathcal{N}_{2}\right)$ is empty.

If $\mathcal{N} \in \Theta\left(\mathcal{M}_{1} \oplus \mathcal{M}_{2}\right)$ with $\mathcal{N} \neq \emptyset$ then there exists a net $\left\{\mathbf{r}^{i} \in \mathcal{M}_{1} \oplus \mathcal{M}_{2}\right\}$ such that $\left\{P_{\mathbf{r}^{i}}(\mathcal{M})\right\}$ converges to $\mathcal{N}$. By going to a subnet, we may assume that $\left\{P_{\mathbf{r}_{1}^{i}}\right\}$ and $\left\{P_{\mathbf{r}_{2}^{i}}\right\}$ converge to $Q_{1} \in \mathcal{E}\left(\Theta\left(\mathcal{M}_{1}\right)\right)$ and $Q_{2} \in \mathcal{E}\left(\Theta\left(\mathcal{M}_{1}\right)\right)$ respectively. Then $\mathcal{N}=Q_{1}\left(\mathcal{M}_{1}\right) \oplus Q_{2}\left(\mathcal{M}_{2}\right)$. It follows that $\widehat{\oplus}$ maps $\Theta\left(\mathcal{M}_{1}\right) \wedge \Theta\left(\mathcal{M}_{2}\right)$ onto $\Theta\left(\mathcal{M}_{1} \oplus \mathcal{M}_{2}\right)$. Since it is injective, it is a homeomorphism.

Similarly, if $Q \in \mathcal{E}\left(\Theta\left(\mathcal{M}_{1} \oplus \mathcal{M}_{2}\right)\right)$ with $Q \neq U$ and so $Q(\mathcal{M}) \neq \emptyset$, we obtain $Q_{1} \in \mathcal{E}\left(\Theta\left(\mathcal{M}_{1}\right)\right)$ and $Q_{2} \in \mathcal{E}\left(\Theta\left(\mathcal{M}_{1}\right)\right)$ so that $Q=Q_{1} \oplus Q_{2}$. It follows that $\widehat{\oplus}$ is surjective between the enveloping semigroups. Furthermore, $Q_{1} \oplus Q_{2}=U$ iff either $Q_{1}=U$ or $Q_{2}=U$, because $Q_{1}\left(\mathcal{M}_{1}\right) \oplus Q_{2}\left(\mathcal{M}_{2}\right)=\emptyset$ iff $Q_{1}\left(\mathcal{M}_{1}\right)$ or $Q\left(\mathcal{M}_{2}\right)$ is empty in which case $\left(\mathcal{Q}_{1} \oplus \mathcal{Q}_{2}\right)\left(\mathcal{M}_{1} \oplus \mathcal{M}_{2}\right)=\emptyset$.

Finally, if $\left(Q_{1}, Q_{2}\right) \neq\left(Q_{1}^{\prime}, Q_{2}^{\prime}\right) \in \mathcal{E}\left(\Theta\left(\mathcal{M}_{1}\right)\right) \times \mathcal{E}\left(\Theta\left(\mathcal{M}_{2}\right)\right)$ with none of the four elements equal to $U$. We may assume that $\mathcal{N}_{1} \in \Theta\left(\mathcal{M}_{1}\right)$ with $Q_{1}\left(\mathcal{N}_{1}\right) \neq Q_{1}^{\prime}\left(\mathcal{N}_{1}\right)$ and $Q_{1}\left(\mathcal{N}_{1}\right) \neq \emptyset$. Since $\widehat{\oplus}$ is injective on $\Theta\left(\mathcal{M}_{1}\right) \wedge \Theta\left(\mathcal{M}_{2}\right)$ it follows that $Q_{1} \oplus Q_{2}\left(\mathcal{N}_{1} \oplus \mathcal{M}_{2}\right) \neq Q_{1}^{\prime} \oplus Q_{2}^{\prime}\left(\mathcal{N}_{1} \oplus \mathcal{M}_{2}\right)$. Hence, $\widehat{\oplus}\left(Q_{1}, Q_{2}\right) \neq \widehat{\oplus}\left(Q_{1}^{\prime}, Q_{2}^{\prime}\right)$ and 
so $\widehat{\oplus}$ is bijective on the enveloping semigroups. We showed above that it is continuous and so is a homeomorphism. It clearly preserves composition and so is a monoid isomorphism.

Corollary 4.26. Assume $\mathcal{M}_{1}$ and $\mathcal{M}_{2}$ are positive disjoint labels. If both $\mathcal{M}_{1}$ and $\mathcal{M}_{2}$ are simple or WAP then $\mathcal{M}_{1} \oplus \mathcal{M}_{2}$ satisfies the corresponding property. If $\mathcal{M}_{1}$ is finite and $\mathcal{M}_{2}$ is semi-simple then $\mathcal{M}_{1} \oplus \mathcal{M}_{2}$ is semi-simple.

Proof: $\mathcal{M}_{1} \oplus \mathcal{M}_{2}=F I N(\mathbb{N})$ then it is simple and WAP. So we may assume $\mathcal{M}_{1} \oplus \mathcal{M}_{2} \neq F I N(\mathbb{N})$.

If $\mathcal{E}\left(\Theta\left(\mathcal{M}_{1}\right)\right)$ and $\mathcal{E}\left(\Theta\left(\mathcal{M}_{2}\right)\right)$ are abelian then $\mathcal{E}\left(\Theta\left(\mathcal{M}_{1}\right)\right) \wedge \mathcal{E}\left(\Theta\left(\mathcal{M}_{2}\right)\right)$ is abelian. So $\mathcal{M}_{1} \oplus \mathcal{M}_{2}$ is WAP when $\mathcal{M}_{1}$ and $\mathcal{M}_{2}$ are by Theorem 4.25.

If every element of $\mathcal{E}\left(\Theta\left(\mathcal{M}_{1}\right)\right)$ other than $U$ is of the form $P_{\mathbf{r}_{1}}$ for some $\mathbf{r}_{1} \in \mathcal{M}_{1}$ and the analogous condition holds for $\mathcal{M}_{2}$ then every element of $\mathcal{E}\left(\Theta\left(\mathcal{M}_{1} \oplus \mathcal{M}_{2}\right)\right)$ is of the form $P_{\mathbf{r}}$ for some $\mathbf{r} \in \mathcal{M}_{1} \oplus \mathcal{M}_{2}$. On the other hand, $U=P_{\mathbf{r}}$ for any $\mathbf{r} \notin \mathcal{M}_{1} \oplus \mathcal{M}_{2}$. Thus, if $\mathcal{M}_{1}$ and $\mathcal{M}_{2}$ are simple then $\mathcal{M}_{1} \oplus \mathcal{M}_{2}$ is.

Assume $\mathcal{M}_{1}$ is finite, and so is simple, and assume $\mathcal{M}_{2}$ is semi-simple. If $Q_{1} \oplus Q_{2} \neq P_{\mathbf{r}}$ then $Q_{2}$ is an external element of $\mathcal{E}\left(\Theta\left(\mathcal{M}_{2}\right)\right)$. Then $Q_{2}\left(\mathcal{M}_{2}\right)$ is finite and so $\left(Q_{1} \oplus Q_{2}\right)\left(\mathcal{M}_{1} \oplus \mathcal{M}_{2}\right) \subset \mathcal{M}_{1} \oplus Q_{2}\left(\mathcal{M}_{2}\right)$ which is finite. Thus, $\mathcal{M}_{1} \oplus \mathcal{M}_{2}$ is semi-simple.

Remark 4.27. If $\mathcal{M}_{2}$ is not simple and $\mathcal{M}_{1}$ is infinite then $\mathcal{M}_{1} \oplus \mathcal{M}_{2}$ is not semi-simple. If $Q \in \mathcal{E}\left(\Theta\left(\mathcal{M}_{2}\right)\right.$ is not equal to some $P_{\mathbf{r}_{2}}$ then $P_{0} \oplus Q$ is not of the form $P_{\mathbf{r}}$ but $\left(P_{0} \oplus Q\right)\left(\mathcal{M}_{1} \oplus \mathcal{M}_{2}\right)=\mathcal{M}_{1} \oplus Q\left(\mathcal{M}_{2}\right)$ which contains $\mathcal{M}_{1}$ and so is infinite.

Example 4.28. Assume $\mathcal{M}_{1}$ and $\mathcal{M}_{2}$ are positive disjoint labels with $\mathcal{M}_{1} \oplus$ $\mathcal{M}_{2} \neq F I N(\mathbb{N})$. If $\mathcal{M}_{1}$ is an infinite simple label and $\mathcal{M}_{2}$ is an infinite finitary label which is not simple, then $\mathcal{M}_{1} \oplus \mathcal{M}_{2}$ is WAP but not semi-simple.

Proposition 4.29. Let $\mathcal{N}$ be a nonempty label and $\left\{\mathcal{M}_{a}\right\}$ be a finite or infinite sequence of positive labels such that $\mathcal{M}_{a} \cap \mathcal{M}_{b} \subset \mathcal{N}$ when $a \neq b$. The label $\mathcal{M}=\bigcup_{a} \mathcal{M}_{a}$ is bounded if all the $\mathcal{M}_{a}$ 's and $\mathcal{N}$ are bounded, and is of finite type if all the $\mathcal{M}_{a}$ 's and $\mathcal{N}$ are of finite type.

If $\mathcal{N}$ is finite and the $\mathcal{M}_{a}$ 's are all finitary then $\mathcal{M}$ is finitary. For example, if all the $\mathcal{M}_{a}$ 's and $\mathcal{N}$ are finite then $\mathcal{M}$ is finitary.

Proof: Let $a \neq b$. If $\rho\left(\mathcal{M}_{a}\right)_{\ell}>\rho\left(\mathcal{M}_{b}\right)_{\ell}$ then $\rho\left(\mathcal{M}_{b}\right)_{\ell} \chi(\ell) \in \mathcal{M}_{a} \cap \mathcal{M}_{b} \subset \mathcal{N}$. Thus, for each $\ell$ there is at most one index $a$ with $\rho\left(\mathcal{M}_{a}\right)_{\ell}>\rho(\mathcal{N})_{\ell}$. Hence, 
$\rho(\mathcal{N})_{\ell}=\max _{a} \rho\left(\mathcal{M}_{a}\right)_{\ell}<\infty$ for all $\ell$ if all the $\mathcal{M}_{a}$ 's and $\mathcal{N}$ are bounded. Hence, in that case $\mathcal{M}=\bigcup_{a} \mathcal{M}_{a}$ is bounded.

If $\mathbf{m}_{a} \in \mathcal{M}_{a}, \mathbf{m}_{b} \in \mathcal{M}_{b}$ with $\mathbf{m}_{a} \geq \mathbf{m}_{b}$ then $\mathbf{m}_{b} \in \mathcal{M}_{a} \cap \mathcal{M}_{b} \subset \mathcal{N}$. Thus, an increasing sequence in $\mathcal{M}$ which is not contained in $\mathcal{N}$ is contained in some $\mathcal{M}_{a}$. Thus, the Finite Chain Condition for $\mathcal{M}$ follows from the condition for $\mathcal{N}$ and each $\mathcal{M}_{a}$.

Now assume that $\mathcal{N}$ is finite and that the $\mathcal{M}_{a}$ 's are finitary. To show that $\mathcal{M}$ is finitary we again apply condition (ii) of Proposition 4.11 (a). Let $\left\{\ell^{i}\right\}$ be a sequence of distinct positive integers. Since $\mathcal{N}$ is finite, $K=\bigcup \operatorname{Supp} \mathcal{N}$ is finite and we may assume, by discarding finitely many elements that $\ell^{i} \notin K$ for all $i$. Since $K$ is finite, it suffices to show that $\{\ell \in \mathbb{N} \backslash K$ : such that eventually $\left.\left\{\ell, \ell^{i}\right\} \in \operatorname{Supp} \mathcal{M}\right\}$ is finite.

If $\ell^{i} \notin \bigcup \operatorname{Supp} \mathcal{M}_{a}$ for any $a$ we let $a_{i}=*$. Otherwise, $\ell^{i} \in \bigcup \operatorname{Supp} \mathcal{M}_{a_{i}}$ for a unique $a_{i}$ since $\ell^{i} \notin K$. If $a_{i}=*$ then $\left\{\ell, \ell^{i}\right\} \notin S u p p \mathcal{M}$ for any $\ell \in \mathbb{N}$. Otherwise,

$$
\begin{aligned}
& \left\{\ell, \ell^{i}\right\} \in \operatorname{Supp} \mathcal{M} \quad \Longleftrightarrow \quad\left\{\ell, \ell^{i}\right\} \in \operatorname{Supp} \mathcal{M}_{a_{i}}, \\
& \left\{\ell, \ell^{i}\right\} \in \operatorname{Supp} \mathcal{M}_{a_{i}} \quad \Longrightarrow \quad\left\{\ell, \ell^{j}\right\} \notin \operatorname{Supp} \mathcal{M} \text { if } a_{j} \neq a_{i} \text {, }
\end{aligned}
$$

because if $a_{j} \neq a_{i}$ then $\ell \notin \bigcup \operatorname{Supp} \mathcal{M}_{a_{j}}$.

If there exists $a$ such that eventually $a_{i}=a$, then eventually $\left\{\ell, \ell^{i}\right\} \in$ Supp $\mathcal{M}$ iff eventually $\left\{\ell, \ell^{i}\right\} \in S u p p \mathcal{M}_{a}$ and the set of such $\ell$ is finite because $\mathcal{M}_{a}$ is finitary.

Otherwise, for no $\ell \in \mathbb{N}$ is it true that eventually $\left\{\ell, \ell^{i}\right\} \in S u p p \mathcal{M}$.

Remark 4.30. Since any label $\mathcal{M}$ is the union of the finite labels $\mathcal{M} \cap \mathcal{B}_{N}$ it follows that some condition is needed to get the finite type or finitary conditions for a union.

From the definition (2.6) of the metric on $\mathcal{L} \mathcal{A B}$ we see that if $\mathcal{M}$ is a nonempty label then

$$
[0, N] \cap \bigcup \operatorname{Supp} \mathcal{M}=\emptyset \quad \Longrightarrow \quad d(0, \mathcal{M}) \leq 2^{-N}
$$

Theorem 4.31. Let $\left\{\mathcal{M}_{a}\right\}$ be a finite or infinite pairwise disjoint collection of positive labels and let $\mathcal{M}=\bigcup\left\{\mathcal{M}_{a}\right\}$. Assume $\mathcal{M} \neq F I N(\mathbb{N})$.

$$
\Theta^{\prime}(\mathcal{M})=\bigcup_{a}\left\{\Theta^{\prime}\left(\mathcal{M}_{a}\right)\right\}
$$

The map sending $P_{\mathbf{r}}$ on $\Theta\left(\mathcal{M}_{a}\right)$ to $P_{\mathbf{r}}$ on $\Theta(\mathcal{M})$ for $\mathbf{0}<\mathbf{r} \in \mathcal{M}_{a}$, extends to a continuous injective homomorphism $j_{a}: \mathcal{A}\left(\Theta\left(\mathcal{M}_{a}\right)\right) \rightarrow \mathcal{A}(\Theta(\mathcal{M}))$. If 
$Q \in \mathcal{A}\left(\Theta\left(\mathcal{M}_{a}\right)\right)$ then

$$
j_{a}(Q)(\mathcal{N})=\left\{\begin{array}{l}
Q\left(\mathcal{M}_{a}\right) \quad \text { if } \mathcal{N}=\mathcal{M}, \\
Q(\mathcal{N}) \quad \text { if } \mathcal{N} \in \Theta^{\prime}\left(\mathcal{M}_{a}\right), \\
\emptyset \quad \text { if } \quad \mathcal{N} \in \Theta^{\prime}\left(\mathcal{M}_{b}\right) \text { with } b \neq a
\end{array}\right.
$$

If $Q_{1} \in \mathcal{A}\left(\Theta\left(\mathcal{M}_{a}\right)\right)$ and $Q_{2} \in \mathcal{A}\left(\Theta\left(\mathcal{M}_{b}\right)\right)$ with $a \neq b$ then $j_{a}\left(Q_{1}\right) j_{b}\left(Q_{2}\right)=$ $j_{b}\left(Q_{2}\right) j_{a}\left(Q_{1}\right)=U$. Furthermore, $\mathcal{A}(\Theta(\mathcal{M})) \backslash \bigcup\left\{j_{a}\left(\mathcal{A}\left(\Theta\left(\mathcal{M}_{a}\right)\right)\right)\right\}$ contains at most one point $Q^{*}$ in which case $Q^{*}(\mathcal{M})=0$ and $Q^{*}=\emptyset$ on $\Theta^{\prime}(\mathcal{M})$ and $Q^{*} Q=Q Q^{*}=U$ for all $Q \in \mathcal{A}(\Theta(\mathcal{M}))$. If some $\mathcal{M}_{a}$ is of finite type then $\mathcal{A}(\Theta(\mathcal{M}))=\bigcup\left\{j_{a}\left(\mathcal{A}\left(\Theta\left(\mathcal{M}_{a}\right)\right)\right)\right\}$.

Proof: If $\left\{\mathbf{r}^{i}>\mathbf{0}\right\}$ is a sequence in $\mathcal{M}$ and $\mathbf{m}>\mathbf{0}$ is an $\mathbb{N}$-vector in $\mathcal{M}$ then $\mathbf{m} \in \mathcal{M}_{a}$ for some $a$. If $\left\{\mathcal{M}-\mathbf{r}^{i}\right\}$ converges with $\mathbf{m}$ in the limit then eventually $\mathbf{r}^{i} \in \mathcal{M}_{a}$ in which case $\mathcal{M}-\mathbf{r}^{i}=\mathcal{M}_{a}-\mathbf{r}^{i}$ and $\operatorname{LIM}\left\{\mathcal{M}-\mathbf{r}^{i}\right\}=\operatorname{LIM}\left\{\mathcal{M}_{a}-\mathbf{r}^{i}\right\} \in$ $\Theta^{\prime}\left(\mathcal{M}_{a}\right)$. Hence, $\Theta^{\prime}(\mathcal{M}) \subset \bigcup\left\{\Theta^{\prime}\left(\mathcal{M}_{a}\right)\right\}$. The reverse inclusion is obvious.

Now suppose that $\left\{P_{\mathbf{r}^{i}}\right\}$ is a net with $\mathbf{r}^{i}>\mathbf{0}$ in $\mathcal{M}$ converging to $Q \in$ $\mathcal{A}(\Theta(\mathcal{M}))$.

Assume first that for some $\mathcal{N} \in \mathcal{A}(\Theta(\mathcal{M}))$ that $\mathbf{0}<\mathbf{m} \in Q(\mathcal{N})$. Let $M_{b}$ be the unique member to the sequence which contains $\mathbf{m}$. Eventually $\mathbf{r}^{i} \in \mathcal{M}_{b}$ so that eventually $\mathcal{M}_{1}-\mathbf{r}^{i}=\left(\mathcal{M}_{1} \cap \mathcal{M}_{b}\right)-\mathbf{r}^{i}$ for all $\mathcal{M}_{1} \in[[M]]$. In particular, $Q(\mathcal{M})=\operatorname{LIM}\left\{\mathcal{M}-\mathbf{r}^{i}\right\}=L I M\left\{\mathcal{M}_{b}-\mathbf{r}^{i}\right\}$.

Notice that $\mathcal{M}_{2}=\bigcup\left\{\mathcal{M}_{2} \cap \mathcal{M}_{a}\right\}$ and by (4.12) $\mathcal{M}_{2} \in \Theta^{\prime}(\mathcal{M})$ iff $\mathcal{M}_{2}=$ $\mathcal{M}_{2} \cap \mathcal{M}_{a} \in \Theta^{\prime}\left(M_{a}\right)$ for some $a$. Clearly $Q\left(\mathcal{M}_{2}\right)=\emptyset$ if $a \neq b$ and $Q\left(\mathcal{M}_{2}\right)=$ $\operatorname{LIM}\left\{\mathcal{M}_{1}-\mathbf{r}_{b}\right\}$ for $\mathcal{M}_{2} \in \Theta\left(M_{a}\right)$. Hence, $Q=j_{b}(\tilde{Q})$ where $\tilde{Q}$ is the pointwise limit of $\left\{P_{\mathbf{r}^{i}}\right\}$ in $\mathcal{A}\left(\Theta\left(\mathcal{M}_{b}\right)\right.$.

On the other hand if $\left\{P_{\mathbf{r}^{i}}\right\}$ in $\mathcal{A}\left(\Theta\left(\mathcal{M}_{b}\right)\right)$ converges to $\tilde{Q}$ with $\mathbf{r}^{i}>\mathbf{0}$ in $\mathcal{M}_{b}$ then $P_{\mathbf{r}^{i}}$ in $\mathcal{A}(\Theta(\mathcal{M}))$ converges pointwise to $Q(\mathcal{N})=L I M\left\{\mathcal{N} \cap \mathcal{M}_{b}-\mathbf{r}^{i}\right\}$ for all $\mathcal{N} \in \Theta(\mathcal{M})$. This defines the injection $j_{b}: \mathcal{A}\left(\Theta\left(\mathcal{M}_{b}\right)\right) \rightarrow \mathcal{A}(\Theta(\mathcal{M}))$ defined by (4.13. It is clear that $j_{b}$ is continuous and is a homomorphism and is obviously injective. Notice that if $\mathbf{r}>0 \in \mathcal{M}_{b}$ then $j_{b}\left(P_{\mathbf{r}}\right)$, the extension of $P_{\mathbf{r}}$ in $\mathcal{A}\left(\Theta\left(\mathcal{M}_{b}\right)\right)$ is just $P_{\mathbf{r}}$ acting on $\Theta(\mathcal{M})$.

Now assume that $Q(\mathcal{M})=0$. If for some cofinal set $\left\{\mathbf{r}^{i^{\prime}} \in \mathcal{M}_{b}\right\}$ then $Q=j_{b}(\tilde{Q})$ with $\tilde{Q}$ the limit in $A\left(\Theta\left(\mathcal{M}_{b}\right)\right)$ of some subnet $\left\{P_{\mathbf{r}_{i^{\prime}}}\right\}$. Otherwise, eventually $\mathbf{r}^{i^{\prime}} \notin M_{b}$ for every $b$ and so $Q(\mathcal{N})=\emptyset$ for all $\mathcal{N} \in \Theta^{\prime}(\mathcal{M})$. Notice that this requires that the sequence $\left\{\mathcal{M}_{a}\right\}$ be infinite. This is $Q^{*} \in \mathcal{A}(\Theta(\mathcal{M})$ with $Q^{*}(\mathcal{M})=0$ and $Q^{*}(\mathcal{N})=\emptyset$ for $\mathcal{N} \in \Theta^{\prime}(\mathcal{M})$. When $\left\{\mathcal{M}_{a}\right\}$ is infinite and $\left\{\mathbf{r}^{i}>0\right\}$ is a sequence in $\mathcal{M}$ with $\mathbf{r}^{i} \in \mathcal{M}_{a(i)}$ and $a(i) \neq a(j)$ for $i \neq j$ then the sequence $\left\{P_{\mathbf{r}^{i}}\right\}$ in $\mathcal{A}(\Theta(\mathcal{M}))$ converges to $Q^{*}$.

It may happen that $Q^{*} \notin \bigcup_{a} j_{a}\left(\mathcal{A}\left(\Theta\left(\mathcal{M}_{a}\right)\right)\right)$, but if $\mathcal{M}_{a}$ is of finite type and $\mathbf{r} \in \max \mathcal{M}_{a}$ then $j_{a}\left(P_{\mathbf{r}}\right)=Q^{*}$ by $(4.2)$. 
Corollary 4.32. Let $\left\{\mathcal{M}_{a}\right\}$ be a finite or infinite pairwise disjoint collection of positive labels and let $\mathcal{M}=\bigcup\left\{\mathcal{M}_{a}\right\}$. If each $\mathcal{M}_{a}$ is semi-simple, finitary or WAP then $\mathcal{M}$ satisfies the corresponding property. If each $\mathcal{M}_{a}$ is simple and at least one $\mathcal{M}_{a}$ is of finite type then $\mathcal{M}$ is simple.

Proof: $F I N(\mathbb{N})$ is simple and WAP. By Proposition 4.29 if the $\mathcal{M}_{a}$ 's are of finite type then $\mathcal{M}$ is of finite type and so is not $F I N(\mathbb{N})$ and if the $\mathcal{M}_{a}$ 's are finitary then $\mathcal{M}$ is finitary. So we may assume $\mathcal{M} \neq F I N(\mathbb{N})$ and apply Theorem 4.31.

In any case, $Q^{*}$ commutes with every element of $\mathcal{E}(\Theta(\mathcal{M}))$ and for $a \neq b$ the elements of $j_{a}\left(\mathcal{A}\left(\Theta\left(\mathcal{M}_{a}\right)\right)\right.$ commute with the elements of $j_{b}\left(\mathcal{A}\left(\Theta\left(\mathcal{M}_{b}\right)\right)\right.$ with $a \neq b$. If each $\mathcal{M}_{a}$ is WAP then each of the $j_{a}\left(\mathcal{A}\left(\Theta\left(\mathcal{M}_{a}\right)\right)\right.$ 's is abelian and so $\mathcal{E}(\Theta(\mathcal{M})$ is abelian, i.e. $\mathcal{M}$ is WAP.

If each $\mathcal{M}_{a}$ is simple then each $Q \in \bigcup_{a} j_{a}\left(\mathcal{A}\left(\Theta\left(\mathcal{M}_{a}\right)\right)\right.$ is of the form $P_{\mathbf{r}}$. If at least one $\mathcal{M}_{a}$ is of finite type then this includes $Q^{*}$.

An external element of $\mathcal{E}(\Theta(\mathcal{M}))$ is either $Q^{*}$ or is $j_{a}(Q)$ for some external $Q \in \mathcal{A}\left(\Theta\left(\mathcal{M}_{a}\right)\right)$. So if all the $\mathcal{M}_{a}$ 's are semi-simple, these all map into the set of finite labels and so $\mathcal{M}$ is semi-simple.

Example 4.33. If $\mathcal{M}=\langle\{\chi(1)+2 \chi(k): k>1\}\rangle$ then $\mathcal{M}$ is simple and so $\emptyset$ is the only recurrent point of $\Theta(\mathcal{M})$. $\mathcal{M}_{k}=\langle\{\chi(1)+\chi(k)\}\rangle=P_{\chi(k)} \mathcal{M}$ and $\mathcal{M}_{1}=$ $\langle\{\chi(1)\}\rangle=P_{2 \chi(k)} \mathcal{M}=P_{\chi(k)} \mathcal{M}_{k}$ for each $k>1$. Since $\mathcal{M}_{1} \cap \mathcal{B}_{N}=\mathcal{M}_{k} \cap \mathcal{B}_{N}$ for $N<k$, it follows that $\mathcal{M}_{1}$ is a non-wandering point of $\Theta(\mathcal{M})$.

Example 4.34. (a) If $\mathcal{M}$ is defined by $\mathcal{M}=\langle\{\chi(1)+\chi(\ell): \ell>1\} \cup\{\chi(\ell)+$ $\chi(\ell+1): \ell>1\}\rangle$, then $\mathcal{M}$ is size bounded and finitary, but not simple.

$$
\begin{gathered}
\mathcal{M}-\chi(1)=\{0\} \cup\{\chi(\ell): \ell>1\}, \\
\mathcal{M}-\chi(\ell)=\{0\} \cup\{\chi(1), \chi(\ell-1), \chi(\ell+1)\}, \quad \text { for } \ell>1, \\
\mathcal{F}=\{0, \chi(1)\}=L I M_{\ell \rightarrow \infty}\{\mathcal{M}-\chi(\ell)\} .
\end{gathered}
$$

Notice that $\mathcal{F} \neq \mathcal{M}-\mathbf{r}$ for any $\mathbf{r} \in \mathcal{M}$.

(b) If $\mathcal{M}$ be defined by $\mathcal{M}=\langle\{\chi(2 a-1)+\chi(2 b): a, b \geq 1\}\rangle$, then $\mathcal{M}$ is size bounded and is simple but is not finitary. In general, if $\mathcal{M}=\mathcal{M}_{1} \oplus \mathcal{M}_{2}$ with $\mathcal{M}_{1}, \mathcal{M}_{2}$ infinite simple labels with $\mathcal{M}_{1} \cap \mathcal{M}_{2}=0$ then $\mathcal{M}$ is simple but not finitary. 
Example 4.35. It can happen that $\mathcal{M}$ is strong finite type but not WAP and so is not semi-simple.

Let $\mathcal{M}$ be defined by $\mathcal{M}=\langle\{\chi(3)+\chi(2 a+1)+\chi(2 b): a \geq b \geq 2\} \cup\{\chi(1)+$ $\chi(3)+\chi(2 b): b \geq 2\}\rangle$.

$$
\begin{gathered}
\mathcal{M}-\chi(1)=\langle\{\chi(3)+\chi(2 b): b \geq 2\}\rangle, \\
\mathcal{M}-\chi(3)=\langle\{\chi(2 a+1)+\chi(2 b): a \geq b \geq 2\} \cup\{\chi(1)+\chi(2 b): b \geq 2\}\rangle \\
\mathcal{M}-\chi(2 \ell+1)=\langle\{\chi(1)+\chi(2 b): \ell \geq b \geq 2\}\rangle \\
\mathcal{M}-\chi(2 \ell)=\langle\{\chi(1)+\chi(2 a+1): a \geq \ell \geq 2\} \cup\{\chi(1)+\chi(3)\}\rangle .
\end{gathered}
$$

It follows that

$$
\begin{aligned}
& L I M_{a \rightarrow \infty}\{\mathcal{M}-\chi(2 a+1)\}=\langle\{\chi(3)+\chi(2 b): b \geq 2\}\rangle, \\
& L I M_{b \rightarrow \infty}\{\mathcal{M}-\chi(2 b)\}=\langle\{\chi(1)+\chi(3)\}\rangle, \\
& L I M_{b \rightarrow \infty} L I M_{a \rightarrow \infty}\{\mathcal{M}-\chi(2 a+1)-\chi(2 b)\}=\{\chi(3), 0\}, \\
& \quad L I M_{a \rightarrow \infty} L I M_{b \rightarrow \infty}\{\mathcal{H}-\chi(2 a+1)-\chi(2 b)\}=\emptyset .
\end{aligned}
$$

The sequences $\left\{P_{\chi(2 a+1)}\right\}$ and $\left\{P_{\chi(2 b)}\right\}$ converge in $\mathcal{E}(\Theta(\mathcal{M}))$ to elements which we will denote by $Q_{o}, Q_{e}$, respectively. $Q_{e}(\mathcal{M})$ is the finite label $\langle\{\chi(1)+$ $\chi(3)\}\rangle \cdot Q_{o}(\mathcal{M})=P_{\chi(1)}(\mathcal{M})$, and so $Q_{e} Q_{o}(\mathcal{M})=P_{\chi(1)}\left(Q_{e}(\mathcal{M})\right)=\langle\{\chi(3)\}\rangle$. On the other hand, $Q_{o} Q_{e}(\mathcal{M})=\emptyset \neq P_{\chi(1)}\left(Q_{e}(\mathcal{M})\right)$. In particular, $Q_{o} \neq P_{\chi(1)}$ on $\Theta(\mathcal{M})$ and so is not continuous.

The enveloping semigroup $\mathcal{E}(\Theta(\mathcal{M}))$ is not abelian and so $\mathcal{M}$ is not WAP. Notice that if $\mathcal{N} \subset \mathcal{M}$ is infinite then $\mathcal{M}-\mathcal{N}$ is finite. Thus, this condition alone does not suffice to yield $\mathcal{M}$ finitary.

We conclude with an example which computes $\mathcal{E}(\Theta(\mathcal{M}))$ for a label $\mathcal{M}$ which is recurrent and so is not WAP.

Example 4.36. For $L \subset \mathbb{N}$ we define the label $\langle L\rangle$ to consist of the characteristic functions of finite subsets of $L$ :

$$
\langle L\rangle=\left\{\chi(F): F \in \mathcal{P}_{f} L\right\}
$$

Since $\chi_{\emptyset}=\mathbf{0},\langle\emptyset\rangle=0$. We compute the orbit space $\Theta(\mathcal{M})$ and the enveloping semigroup $\mathcal{E}(\Theta(\mathcal{M}))$ for the recurrent label $\mathcal{M}=\langle\mathbb{N}\rangle$. Since $\mathcal{M}$ is recurrent, it is not WAP and so the semigroup cannot be abelian.

We will show that

$$
\Theta(\mathcal{M})=\{\emptyset\} \cup\{\langle L\rangle: L \subset \mathbb{N}\} .
$$

Let $2^{\beta \mathbb{N}} \cup\{*\}$ be the compact space of all compact subsets of $\beta \mathbb{N}$ together with an additional isolated point, labeled $*$. We will apply the results about 
$2^{\beta \mathbb{N}}$ which are reviewed in Appendix C. In particular, for $A \in 2^{\beta \mathbb{N}}, \mathcal{F}_{A}=\{L \subset$ $\mathbb{N}: A \subset \bar{L}\}$ and $A_{0}=A \cap \mathbb{N}$. Thus, $\mathcal{F}_{\emptyset}$ is the power set of $\mathbb{N}$. If $A$ is nonempty then $\mathcal{F}_{A}$ is a filter of subsets of $\mathbb{N}$ by Proposition C.3 (a).

For $A \in 2^{\beta \mathbb{N}} \cup\{*\}$ we define $Q_{A}: \Theta(\mathcal{M}) \rightarrow \Theta(\mathcal{M})$ by $Q_{A}(\emptyset)=\emptyset$ for all $A$ and $Q_{*}(\langle L\rangle)=\emptyset$ for all $L \subset \mathbb{N}$ and for $A \in 2^{\beta \mathbb{N}}, L \subset \mathbb{N}$ :

$$
Q_{A}(\langle L\rangle)=\left\{\begin{array}{l}
\left\langle L \backslash A_{0}\right\rangle \text { for } L \in \mathcal{F}_{A}, \\
\emptyset \quad \text { otherwise. }
\end{array}\right.
$$

Thus, $Q_{*}=U$, the retraction to the fixed point $\emptyset$.

We will show that $A \mapsto Q_{A}$ defines a homeomorphism from $2^{\beta \mathbb{N}} \cup\{*\}$ onto $\mathcal{E}(\Theta(\mathcal{M}))$. Composition is described by

$$
Q_{B} Q_{A}= \begin{cases}Q_{A \cup B} & \text { if } \mathbb{N} \backslash A_{0} \in \mathcal{F}_{B}, \\ U & \text { otherwise. }\end{cases}
$$

for $A, B \in 2^{\beta \mathbb{N}}$. Notice that $\mathbb{N} \backslash A_{0} \in \mathcal{F}_{B}$ requires $A_{0} \cap B_{0}=\emptyset$. If a finite set $F \subset \mathbb{N}$ is disjoint from $B_{0}$ then $\mathbb{N} \backslash F \in \mathcal{F}_{B}$. So if $A_{0}$ is finite and $A_{0} \cap B_{0}=\emptyset$ then $\mathbb{N} \backslash A_{0} \in \mathcal{F}_{B}$. In general, $\mathbb{N} \backslash A_{0} \in \mathcal{F}_{B}$ iff the clopen set $\overline{A_{0}}$ is disjoint from $B$.

From this it is easy to check that for $A, B \in 2^{\beta \mathbb{N}}$

$$
\begin{gathered}
Q_{A} Q_{A}= \begin{cases}Q_{A} & \text { if } A_{0}=\emptyset, \\
U & \text { otherwise. }\end{cases} \\
Q_{B} Q_{A}=Q_{A \cup B}=Q_{A} Q_{B} \text { if } \mathbb{N} \backslash A_{0} \in \mathcal{F}_{B}, \text { and } \mathbb{N} \backslash B_{0} \in \mathcal{F}_{A} . \\
Q_{B} Q_{A}=U=Q_{A} Q_{B} \text { if } \mathbb{N} \backslash A_{0} \notin \mathcal{F}_{B}, \text { and } \mathbb{N} \backslash B_{0} \notin \mathcal{F}_{A} .
\end{gathered}
$$

Thus, if $A_{0}=\emptyset, Q_{A}$ is an idempotent which fixes $\langle L\rangle$ for all $L \in \mathcal{F}_{A}$. In particular, $Q_{A}(\mathcal{M})=\mathcal{M}$ and thus $\mathcal{M}$ is recurrent. The failure of commutativity occurs only when $\overline{A_{0}}$ is disjoint from $B$, and so $B_{0}$ is disjoint from $A$, but $\overline{B_{0}}$ meets $A$. In that case, $Q_{B} Q_{A}=Q_{A \cup B}$, but $Q_{A} Q_{B}=U$.

Now we prove all this.

Proof: Let $\Theta=\{\emptyset\} \cup\{\langle L\rangle: L \subset \mathbb{N}\}$. Notice that each $\langle L\rangle$ is a lattice and $\Theta$ is the collection of all sublattices of $\langle\mathbb{N}\rangle$, see Proposition 2.22 (e). The set $\left\{\mathcal{N}: \mathbf{r}_{1}, \mathbf{r}_{2} \in \mathcal{N}\right.$, and $\left.\mathbf{r}_{1} \vee \mathbf{r}_{2} \notin \mathcal{N}\right\}$ is clopen for every pair $\mathbf{r}_{1}, \mathbf{r}_{2} \in F \operatorname{IN}(\mathbb{N})$. The union over all pairs is the -open- complement of the set of sublattices of $F I N(\mathbb{N})$.

If $F \subset \mathbb{N}$ is finite then $P_{\chi(F)}(\mathcal{M})=\langle\mathbb{N} \backslash F\rangle$ which is a sublattice. Since the set of sublattices is closed, it follows that $\Theta(\mathcal{M}) \subset \Theta$. 
Next we show that if $\left\{A^{i}\right\}$ is a net in $2^{\beta \mathbb{N}} \cup\{*\}$ converging to $A$ then $\left\{Q_{A^{i}}\right\}$ converges pointwise to $Q_{A}$ as a function on $\Theta$. Since $*$ and $\emptyset$ are isolated points we may assume that $A$ and all the $A^{i}$ 's are nonempty elements of $2^{\beta \mathbb{N}}$.

If $L \notin \mathcal{F}_{A}$ then by Proposition C.6 eventually $L \notin \mathcal{F}_{A^{i}}$ and so eventually $Q_{A^{i}}(\langle L\rangle)=\emptyset=Q_{A}(\langle L\rangle)$. If $L \in \mathcal{F}_{A}$ then by Proposition C.6 again eventually $L \notin \mathcal{F}_{A^{i}}$ and so eventually $Q_{A^{i}}(\langle L\rangle)=\left\langle L \backslash\left(A^{i}\right)_{0}\right\rangle$ and $Q_{A}(\langle L\rangle)=\left\langle L \backslash A_{0}\right\rangle$. Furthermore, for the finite set $[1, \ell]$ eventually $[1, \ell] \cap\left(A^{i}\right)_{0}=[1, \ell] \cap A_{0}$. This implies that $\left\{\left\langle L \backslash\left(A^{i}\right)_{0}\right\rangle\right\}$ converges to $\left\langle L \backslash A_{0}\right\rangle$ in $\mathcal{L} \mathcal{A} \mathcal{B}$.

For any $A \in 2^{\beta \mathbb{N}}$ there is a net $\left\{F^{i}\right\}$ of finite subsets of $\mathbb{N}$ with limit $A$, see Corollary C.5 (b). So $\left\{Q_{F^{i}}\right\}$ converges to $Q_{A}$ pointwise. In particular, $P_{\chi\left(F^{i}\right)}(\mathcal{M})=Q_{F^{i}}(\mathcal{M}) \rightarrow Q_{A}(\langle\mathbb{N}\rangle)=\left\langle\mathbb{N} \backslash A_{0}\right\rangle$. If $L \subset \mathbb{N}$ and $A=\overline{\mathbb{N} \backslash L}$ with $A_{0}=\mathbb{N} \backslash L$ it follows that $Q_{A}(\langle\mathbb{N}\rangle)=\langle L\rangle \in \Theta(\mathbb{N})$. Hence, $\Theta(\mathcal{M})=\Theta$ and every $Q_{A} \in \mathcal{E}(\Theta(\mathcal{M}))$. If $F \subset \mathbb{N}$ is finite then clearly $Q_{F}=P_{\chi(F)}$ on $\Theta(\mathcal{M})$. In particular, $Q_{\emptyset}=P_{\mathbf{0}}=i d_{\Theta(\mathcal{M})}$. If $\mathbf{r} \in F I N(\mathbb{N}) \backslash\langle\mathbb{N}\rangle$ then $P_{\mathbf{r}}=U=Q_{*}$.

Now suppose that $\left\{P_{\mathbf{r}^{i}}\right\}$ converges to $Q \in \mathcal{E}(\Theta(\mathcal{M}))$. If $\mathbf{r}^{i} \notin\langle\mathbb{N}\rangle$ for some cofinal set then $Q=U$. So we may assume that $\mathbf{r}^{i} \in\langle\mathbb{N}\rangle$ for all $i$. Then for each $i$ there is a finite set $F^{i}$ such that $\mathbf{r}^{i}=\chi\left(F^{i}\right)$. By going to a subnet, we may assume that $\left\{F^{i}\right\}$ converges to $A \in \beta \mathbb{N}$. Then $\left\{Q_{F^{i}}=P_{\mathbf{r}^{i}}\right\}$ converges to $Q_{A}$ and so $Q=Q_{A}$. Thus, the continuous map $A \rightarrow Q_{A}$ from $2^{\beta \mathbb{N}} \cup\{*\}$ to $\mathcal{E}(\Theta(\mathcal{M}))$ is surjective. If $A, B \in 2^{\beta \mathbb{N}}$ are unequal then we may assume that $B \backslash A \neq \emptyset$. Then there exists $L \subset A$ such that the clopen set $\bar{L}$ contains $A$ but $B$ meets its complement. Hence, $L \in \mathcal{F}_{A}$ which $L \notin \mathcal{F}_{B}$. So $Q_{B}(\langle L\rangle)=$ $\emptyset \neq\left\langle L \backslash A_{0}\right\rangle=Q_{A}(\langle L\rangle)$. It follows that the map $A \mapsto Q_{A}$ is injective and so is a homeomorphism from the compact space $2^{\beta \mathbb{N}} \cup\{*\}$ onto $\mathcal{E}(\Theta(\mathcal{M}))$.

Now let $A, B \in 2^{\beta \mathbb{N}}$. If $L \in \mathcal{F}_{A}$ then $Q_{A}(\langle L\rangle)=\left\langle L \backslash A_{0}\right\rangle$. So if $L \backslash A_{0} \in \mathcal{F}_{B}$, which requires $A_{0} \cap B_{0}=\emptyset$, then we have $Q_{B} Q_{A}(\langle L\rangle)=\left\langle\left(L \backslash A_{0}\right) \backslash B_{0}\right\rangle=\langle L \backslash$ $\left.(A \cup B)_{0}\right\rangle$. Since $\mathcal{F}_{B}$ is a filter $L \backslash A_{0} \in \mathcal{F}_{B}$ iff $L, \mathbb{N} \backslash A_{0} \in \mathcal{F}_{B}$. On the other hand, $L \in \mathcal{F}_{A}$ and $L \in \mathcal{F}_{B}$ iff $L \in \mathcal{F}_{A \cup B}$ in which case $Q_{A \cup B}(\langle L\rangle)=\left\langle L \backslash(A \cup B)_{0}\right\rangle$. If $L \notin \mathcal{F}_{A \cup B}$ then either $L \notin \mathcal{F}_{A}$ and so $Q_{B} Q_{A}(\langle L\rangle)=Q_{B}(\emptyset)=\emptyset=Q_{A \cup B}(\langle L\rangle)$, or $L \in \mathcal{F}_{A}$ but $L \notin \mathcal{F}_{B}$ so the $Q_{B} Q_{A}(\langle L\rangle)=Q_{B}\left(\left\langle L \backslash A_{0}\right\rangle\right)=\emptyset$. It follows that if $\mathbb{N} \backslash A_{0} \in \mathcal{F}_{B}$ then $Q_{B} Q_{A}=Q_{A \cup B}$. On the other hand, if $\mathbb{N} \backslash A_{0} \notin \mathcal{F}_{B}$ then $Q_{A}(\langle L\rangle)=\emptyset$ and so $Q_{B} Q_{A}(\langle L\rangle)=\emptyset$ or $Q_{A}(\langle L\rangle)=\left\langle L \backslash A_{0}\right\rangle$ and so $Q_{B} Q_{A}(\langle L\rangle)=\emptyset$ since $L \backslash A_{0} \notin \mathcal{F}_{B}$. Thus, if $\mathbb{N} \backslash A_{0} \notin \mathcal{F}_{B}$ then $Q_{B} Q_{A}=U$.

The (4.21) results follow easily from (4.20).

\section{Dynamical Properties of $X(\mathcal{M})$}

\subsection{Translation finite subsets of $\mathbb{Z}$.}


We recall the following combinatorial characterization of WAP subsets of $\mathbb{Z}$ $([37])$.

Theorem 5.1 (Ruppert). For a subset $A \subset \mathbb{Z}$ the following conditions are equivalent:

(1) The subshift $\overline{\mathcal{O}}(\chi(A)) \subset\{0,1\}^{\mathbb{Z}}$ is WAP.

(2) For every infinite subset $B \subset \mathbb{Z}$ either:

(i) there exists $N \geq 1$ such that

$$
\bigcap_{b \in B \cap[-N, N]} A-b \text { is finite, }
$$

or:

(ii) there exists $N \geq 1$ and $n \in \mathbb{Z}$ such that

$$
A-n \supset B \cap(\mathbb{Z} \backslash[-N, N]) .
$$

Definition 5.2 (Ruppert). We say that a subset $A \subset \mathbb{Z}$ is translation finite (TF hereafter) if for every infinite subset $B \subset \mathbb{Z}$ there exists an $N \geq 1$ such that

$$
\bigcap_{b \in B \cap[-N, N]} A-b=\{n \in \mathbb{N}: A-n \supset B \cap[-N, N]\} \quad \text { is finite. }
$$

Example 5.3. It is easy to check that the set $A=2 \mathbb{Z}_{+} \cup-\left(2 \mathbb{Z}_{+} 1\right)$

(with $c=\chi(A)=(\ldots, 1,0,1,0,1, \dot{1}, 0,1,0, \ldots)$ ) does not satisfy Ruppert's condition (and a fortiori is not translation finite), hence $\overline{\mathcal{O}}(\chi(A))$ is not WAP.

(See Example 1.18.(b).)

Proposition 5.4. Let $A$ be a subset of $\mathbb{Z}$. The following conditions are equivalent.

(1) The subset $A$ is TF.

(2) Every point in $R_{S}(\chi(A))=\left(\omega_{S} \cup \alpha_{S}\right)(\chi(A))$ has finite support.

(3) The subshift $\overline{\mathcal{O}}(\chi(A))$ is $C$ T of height at most 2.

Proof: $(1) \Longrightarrow(2)$ : Suppose first that $A$ is TF and suppose that for some sequence $\left\{n_{i}\right\}_{i=1}^{\infty}$, with $\left|n_{i}\right| \rightarrow \infty$, we have $S^{n_{i}} \chi(A)=x$ with supp $x$ an infinite set. Let $B=\operatorname{supp} x$ and observe that for every $N \geq 1$, eventually,

$$
S^{n_{i}} \chi(A) \wedge[-N, N]=\left(x_{-N}, \ldots, x_{N}\right),
$$

whence $A-n_{i} \supset B \cap[-N, N]$. But this contradicts our assumption that $A$ is TF. 
$(2) \Rightarrow(1)$ : Conversely, suppose $A$ is not TF. Then there exists an infinite $B \subset \mathbb{Z}$ such that for every $N \geq 1$ the intersection

$$
\{n \in \mathbb{Z}: A-n \supset B \cap[-N, N]\} \quad \text { is infinite. }
$$

We can construct a strictly increasing sequence $\left\{n_{i}\right\}_{i=1}^{\infty}$ with $A-n_{i} \supset B \cap$ $[-i, i]$ and so for any limit point $x \in\{0,1\}^{\mathbb{Z}}$ of the sequence $\left\{S^{n_{i}} \chi(A)=\right.$ $\left.\chi\left(A-n_{i}\right)\right\}$ the support supp $x \supset B$ and so is infinite.

$(2) \Rightarrow(3)$ : is obvious.

$(3) \Rightarrow(2)$ : Suppose finally that that $\overline{\mathcal{O}}(\chi(A))$ is CT of height at most 2 . Suppose to the contrary that $x \in\left(\omega_{T} \cup \alpha_{T}\right)(\chi(A))$ has infinite support, say supp $x=B$. By compactness there exists a sequence $\left\{n_{i}\right\}_{i=1}^{\infty} \subset B$ such that the sequence $S^{n_{i}} x$ converges. Let $y=\lim _{i \rightarrow \infty} S^{n_{i}} x$. Then $y \in R_{T}(x)$ and $y_{0}=1$. Thus $y \neq \mathbf{0}$ and this contradicts our assumption that $\overline{\mathcal{O}}(\chi(A))$ is of height at most 2 .

We next address the question 'when is $A[\mathcal{M}]$ TF ?'. This turns out to be a rather restrictive condition, because $\emptyset$ and 0 are the only labels $\mathcal{N}$ such that $A[\mathcal{N}]$ has finite support. For $\mathcal{M}=\emptyset$ or $0, R_{S}(A[\mathcal{N}])=\{e\}$ where $e$ is the fixed point $\overline{0}=A[\emptyset]$. Thus, in these cases $x[\mathcal{M}]$ is TF.

Proposition 5.5. For a positive label $\mathcal{M}$ the following conditions are equivalent.

(i) $\Theta(\mathcal{M})=\{\mathcal{M}, 0, \emptyset\}$.

(ii) For all $\mathbf{r}>\mathbf{0} \in \mathcal{M}, \mathcal{M}-\mathbf{r}=0$.

(iii) There exists $L$ a nonempty subset of $\mathbb{N}$ such that $\mathcal{M}=\langle\{\chi(\ell): \ell \in$ $L\}\rangle$.

(iv) $A[\mathcal{M}]$ is $T F$.

(v) $(X(\mathcal{M}), S)$ has height 2.

(vi) $\left(X_{+}(\mathcal{M}), S\right)$ has height 2.

When these conditions hold, $\mathcal{M}$ is finitary and simple.

Proof: (iii) $\Rightarrow$ (ii) : Obvious.

(ii) $\Rightarrow$ (i) : From (ii) it is clear that $\Theta(\mathcal{M})$ consists of $P_{\mathbf{r}}(\mathcal{M})=\emptyset$ for $\mathbf{r} \notin \mathcal{M}$ and $P_{\mathbf{r}}(\mathcal{M})=0$ for $\mathbf{r}>\mathbf{0} \in \mathcal{M}$ and finally, $P_{\mathbf{0}}(\mathcal{M})=\mathcal{M}$. Hence, the only limit labels possible in $\Theta(\mathcal{M})$ are $\emptyset, 0$ and $\mathcal{M}$. In passing, we see that $\mathcal{A}(\Theta(\mathcal{M}))$ contains one nontrivial element which maps $\mathcal{M}$ to 0 and maps 0 and $\emptyset$ to $\emptyset$.

(i) $\Rightarrow$ (iii) : If $\mathbf{r} \in \mathcal{M}$ with $|\mathbf{r}| \geq 2$ then there exists $\ell \in \mathbb{N}$ such that $\mathbf{r}-\chi(\ell)>\mathbf{0}$. Hence, $\mathcal{M}-\chi(\ell) \in \Theta(\mathcal{M})$ is neither 0 nor $\emptyset$. Hence, $\mathcal{M}-\chi(\ell)=\mathcal{M}$. That is, $\mathbf{r}+\chi(\ell) \in \mathcal{M}$ for all $\mathbf{r} \in \mathcal{M}$. So $\mathbf{r} \in \mathcal{M}$ implies $\chi(\ell) \in \mathcal{M}-\mathbf{r}$ and thus, $\mathcal{M}-\mathbf{r}=\mathcal{M}$ for all $\mathbf{r} \in \mathcal{M}$. This implies that $0 \notin \Theta(\mathcal{M})$. Contrapositively, (i) implies that $|\mathbf{r}|=1$ for any nonzero $\mathbf{r}$ in $\mathcal{M}$. That is, each nonzero $\mathbf{r}$ in $\mathcal{M}$ is some $\chi(\ell)$. 
(iii) $\Rightarrow(\mathrm{v}): \mathcal{M}$ is bounded and size-bounded and so is of finite type. By Corollary 3.43 the limit points of $x[\mathcal{M}]$ lie on the orbits of $x[\mathcal{N}]$ for some $\mathcal{N} \in \Theta(\mathcal{M})$. Since, $\mathcal{M}$ is not recurrent, the set $R_{S}(x[\mathcal{M}])$ of limit points consists of the orbits of $x[0]$ and $x[\emptyset]$. These in turn map to the fixed point $x[\emptyset]$ and so $(X(\mathcal{M}), S)$ has height 2 .

(v) $\Rightarrow$ (iv) : This follows from Proposition 5.4.

(iv) $\Rightarrow$ (iii) : We prove the contrapositive, assuming, as above, that there exist $\mathbf{r} \in \mathcal{M}$ and $\ell \in \mathbb{N}$ such that $\mathbf{r}-\chi(\ell)>\mathbf{0}$. Choose an increasing sequence $\left\{t^{i} \in I P(k)\right\}$ with length vectors $\mathbf{r}\left(t^{i}\right)=\chi(\ell)$ and with $\left|j_{r}\left(t^{i}\right)\right| \rightarrow \infty$. By $\left\{S^{t^{2}}(x[\mathcal{M}])\right\}$ converges to $x[\mathcal{M}-\chi(\ell)]$ which does not have finite support since $\mathbf{r}-\chi(\ell) \in \mathcal{M}-\chi(\ell)$. By Proposition 5.4 again $A[\mathcal{M}]$ is not TF.

Finally, it is clear that the labels described in (iii) are finitary and simple.

\subsection{Non-null and non-tame labels.}

Definition 5.6. (a) For a subshift $(X, S)$ a subset $K \subset \mathbb{Z}$ is called an independent set if the restriction to $X$ of the projection $\pi_{K}:\{0,1\}^{\mathbb{Z}} \rightarrow\{0,1\}^{K}$ is surjective. The subshift is called null if there is a finite bound on the size of the independent sets for $(X, S)$. It is called tame if there is no infinite independent set for $(X, S)$.

(b) For a label $\mathcal{M}$ a subset $L \subset \mathcal{M}$ is called an independent set if for every $L_{1} \subset L$ there exists $\mathcal{N} \in \Theta(\mathcal{M})$ such that $L \cap \mathcal{N}=L_{1}$.

(c) A label $\mathcal{M}$ is called non-null if for every $n \in \mathbb{N}$ there is a finite independent subset $F \subset \mathcal{M}$ with $\# F \geq n$. It is non-tame if there is an infinite set $L \subset \mathcal{M}$ such that every finite $F \subset L$ is an independent set.

Notice that an independent set $L$ for a label $\mathcal{M}$ is certainly not a label. In fact, if $\mathbf{m}_{1}<\mathbf{m}$ and $\mathbf{m} \in L$ then $\mathbf{m}_{1} \notin L$ because if $\mathbf{m} \in \mathcal{N}$ for a label $\mathcal{N}$ then $\mathbf{m}_{1} \in \mathcal{N}$. Since there exists a label $\mathcal{N}$ such that $\mathcal{N} \cap L=\{\mathbf{m}\}$ it follows that $\mathbf{m}_{1} \notin L$.

Remark 5.7. The concepts 'null' and 'tame' are defined for any dynamical system. The first is defined in terms of sequential topological entropy (see e.g. [29] and the review [28]) and the latter in terms of the dynamical BourgainFremlin-Talagrand dichotomy for enveloping semigroups ([19]). The convenient criteria which we use here for subshifts to be non-null and non-tame, are due basically to Kerr and Li [35] (see [22, Theorem 6.1.(3)]). 
Proposition 5.8. $(X(F I N(\mathbb{N})), S)$ is not tame.

Proof: We have $X(F I N(\mathbb{N}))=X(I P(k))$. Consider the sequence $\left\{a_{n}=\right.$ $k(2 n)+k(2 n-1): n \geq 1\}$. Let $u=\left\{u_{j}\right\}_{j \geq 1}$ be a sequence of 0 's and 1's and let $s(u, n)=\sum\left\{k(2 i-1): u_{i}=1, i \leq n\right\}$. Then $s(u, n)+a_{j}$, for $1 \leq j \leq n$, is in $I P(k)$ if and only if $u_{j}=0$ and thus we can interpolate on any finite initial segment of the sequence $\left\{a_{n}\right\}_{n \geq 1}$. It follows that this infinite sequence is an independent set for $(X(F I N(\mathbb{N})), S)$.

Lemma 5.9. (a) If $\mathcal{M}$ is a label and $F$ is a finite subset of $\mathcal{M}$ then for any $\mathcal{N} \in \Theta(\mathcal{M})$ there exists an $\mathbb{N}$-vector $\mathbf{r}$ such that $\mathcal{N} \cap F=\mathcal{M}-\mathbf{r} \cap F$. In particular, if $F$ is a finite independent subset of $\mathcal{M}$ then for every $A \subset F$ there exists $\mathbf{r}$ such that $F \cap \mathcal{M}-\mathbf{r}=A$.

(b) If every finite subset $F \subset L$ is an independent set for a label $\mathcal{M}$ then $L$ is an independent set for $\mathcal{M}$.

(c) If $L$ is an independent set for a label $\mathcal{M}$, and if for every $\mathbf{m} \in L, t(\mathbf{m}) \in$ $I P_{+}(k)$ such that $\mathbf{r}(t(\mathbf{m}))=\mathbf{m}$ then $K=\{t(\mathbf{m}): \mathbf{m} \in L\}$ is an independent set for the subshifts $(X(\mathcal{M}), S)$ and $\left(X_{+}(\mathcal{M}), S\right)$.

Proof: (a) If $\mathcal{B}_{N}$ contains all the supports of elements of $F$ then $\mathbf{m} \in F$ is in $\mathcal{M}_{1} \in \mathcal{L} \mathcal{A B}$ iff it is in $\mathcal{M}_{1} \cap \mathcal{B}_{N}$. Hence, for any $A \subset F$ the set $\left\{\mathcal{M}_{1}\right.$ : $\left.\mathcal{M}_{1} \cap F=A\right\}$ is clopen in $\mathcal{L} \mathcal{A} \mathcal{B}$. Since $\{\mathcal{M}-\mathbf{r}\}$ is dense in $\Theta(\mathcal{M})$, the result follows.

(b) Let $L_{1} \subset L$. Let $\left\{F^{i}\right\}$ be an increasing sequence of finite subsets of $L$ with union $L$. Because $F^{i}$ is an independent set, part (a) implies there exists $\mathbf{r}^{i}$ such that $F^{i} \cap \mathcal{M}-\mathbf{r}^{i}=L_{1} \cap F^{i}$. It follows that if $\mathbf{m} \in L_{1}$ then eventually $\mathbf{m} \in \mathcal{M}-\mathbf{r}^{i}$. If $\mathbf{m} \in L \backslash L_{1}$ then eventually $\mathbf{m} \notin \mathcal{M}-\mathbf{r}^{i}$. By going to a subsequence, we can assume that $\left\{\mathcal{M}-\mathbf{r}^{i}\right\}$ converges to some $\mathcal{N} \in \Theta(\mathcal{M})$. Clearly, $L \cap \mathcal{N}=L_{1}$.

(c) For any label $\mathcal{N} \in \Theta(\mathcal{M}), \quad t \in A[\mathcal{N}]$ iff $t \in I P(k)$ with $\mathbf{r}(t) \in \mathcal{N}$ and so $x[\mathcal{N}]_{t}=1$ iff $\mathbf{r}(t) \in \mathcal{N}$ and similarly $t \in A_{+}[\mathcal{N}]$ iff $t \in I P_{+}(k)$ with $\mathbf{r}(t) \in \mathcal{N}$ and so $x_{+}[\mathcal{N}]_{t}=1$ iff $\mathbf{r}(t) \in \mathcal{N}$.

For $K_{1} \subset K$, let $L_{1}=\left\{\mathbf{r}(t): t \in K_{1}\right\}=\left\{\mathbf{m}: t(\mathbf{m}) \in K_{1}\right\} \subset L$. Since $L$ is an independent set for $\mathcal{M}$, there exists $\mathcal{N} \in \Theta(\mathcal{M})$ such that $L \cap \mathcal{N}=L_{1}$. Hence, $x[\mathcal{N}]_{t}=1$ for $t \in K_{1}$ and $=0$ for $t \in K \backslash K_{1}$. Thus, $K$ is an independent set for $X(\mathcal{M})$. Since $K \subset I P_{+}(k)$ the same argument works for $X_{+}(\mathcal{M})$.

From this we obviously have

Proposition 5.10. (a) A label $\mathcal{M}$ is non-null iff for every $n \in \mathbb{N}$ there is a finite subset $F \subset \mathcal{M}$ with $\# F \geq n$ such that for every $A \subset F$ there exists $\mathbf{r}$ such that $F \cap \mathcal{M}-\mathbf{r}=A$. 
(b) A label $\mathcal{M}$ is non-tame if there is an infinite set $L \subset \mathcal{M}$ such that for any finite $A \subset F \subset L$ there exists $\mathbf{r}$ such that $F \cap \mathcal{M}-\mathbf{r}=A$. In that case if $L_{1}$ is any subset of $L$ then there exists $\mathcal{N} \in \Theta(\mathcal{M})$ such that $L \cap \mathcal{N}=L_{1}$. In particular, $\Theta(\mathcal{M})$ is uncountable.

Recall that $\operatorname{Supp} \mathcal{M}$ f-contains $L$ when $\mathcal{P}_{f} L \subset S u p p \mathcal{M}$, i.e. every finite subset of $L$ is the support of an element of $\mathcal{M}$, or, equivalently, if $\langle\chi(L)\rangle \subset \mathcal{M}$. By Proposition 2.5 if a label f-contains an infinite set then it is not of finite type and the converse holds if the label is bounded and not of finite type then it f-contains an infinite set.

Remark 5.11. It follows that if $\mathcal{M}$ is a non-tame label then $X(\mathcal{M})$ and $X_{+}(\mathcal{M})$ are uncountable and so neither $(X(\mathcal{M}), S)$ nor $\left(X_{+}(\mathcal{M}), S\right)$ can be WAP.

Corollary 5.12. Given a label $\mathcal{M}$, if any label $\mathcal{N} \in \Theta(\mathcal{M})$ is non-null (or non-tame) then the subshifts $(X(\mathcal{M}), S),\left(X_{+}(\mathcal{M}), S\right)$ are not null (resp. not tame).

Proof: If $\mathcal{N} \in \Theta(\mathcal{M})$ then $X(\mathcal{N}) \subset X(\mathcal{M})$ and so if $X(\mathcal{N})$ projects onto $\{0,1\}^{L}$ then $X(\mathcal{M})$ does.

There are some simple conditions which allow us to find non-tame labels.

Definition 5.13. A bounded label $\mathcal{M}$ with roof $\rho(\mathcal{M})$ is called flat over a set $L \subset \mathbb{N}$ if for all $F \in S u p p \mathcal{M}$ with $F \subset L, \rho(\mathcal{M}) \mid F \in \mathcal{M}$. Equivalently, if $\mathbf{m} \in \mathcal{M}$ with supp $\mathbf{m} \subset L$ then $\rho(\mathcal{M}) \mid(\operatorname{supp} \mathbf{m}) \in \mathcal{M}$. The label is called flat when it is flat over $\mathbb{N}$. So a flat label is bounded.

Lemma 5.14. Let $L \subset \mathbb{N}$.

(a) If the label $\mathcal{M}$ is flat over $L$ and $\mathbf{r}$ is an $\mathbb{N}$-vector with supp $\mathbf{r} \subset L$ then $\mathcal{M}-\mathbf{r}$ is flat over $L$.

(b) If $\left\{\mathcal{M}^{i}\right\}$ is a collection of labels each flat over $L$ then $\bigcap\left\{\mathcal{M}^{i}\right\}$ is a label which is flat over L.

(c) If $\left\{\mathcal{M}^{i}\right\}$ is an increasing sequence of labels flat over $L$ and $\bigcup\left\{\mathcal{M}^{i}\right\}$ is bounded, then it is a label flat over $L$.

(d) If $\left\{\mathcal{M}^{i}\right\}$ is a sequence of labels flat over $L$ and $\bigcup\left\{\mathcal{M}^{i}\right\}$ is bounded, then LIMINF $\left\{\mathcal{M}^{i}\right\}$ is a label flat over $L$.

(e) The set of labels which are flat is a closed in the subset of bounded labels.

(f) If $\mathcal{M}$ is a flat label then the elements of $\Theta(\mathcal{M})$ are all flat labels. 
Proof: (a) If $\mathbf{m} \in \mathcal{M}-\mathbf{r}$ with supp $\mathbf{m} \subset L$ and $F=\operatorname{supp}(\mathbf{m}+\mathbf{r})$ then $F \subset L$ and so $\rho(\mathcal{M}) \mid F \in \mathcal{M}$. Hence, $\rho(\mathcal{M}) \mid F-\mathbf{r} \in \mathcal{M}-\mathbf{r}$ and $\rho(\mathcal{M}) \mid F-\mathbf{r}=$ $(\rho(\mathcal{M})-\mathbf{r}) \mid F$. Clearly, $\rho(\mathcal{M})-\mathbf{r} \geq \rho(\mathcal{M}-\mathbf{r})$.

(b) If $\mathcal{M}$ is the intersection then $\rho(\mathcal{M})=\min \left\{\rho\left(\mathcal{M}^{i}\right)\right\}$. If $\mathbf{m} \in \mathcal{M}$ with supp $\mathbf{m} \subset L$ then $\rho(\mathcal{M}) \mid(\operatorname{supp} \mathbf{m}) \in \mathcal{M}^{i}$ for all $i$ and so is in $\mathcal{M}$.

(c) If $\mathcal{M}$ is the union then $\rho(\mathcal{M})=\max \left\{\rho\left(\mathcal{M}^{i}\right)\right\}$ and this is a non-decreasing sequence of functions. On any finite set $F$, eventually $\rho(\mathcal{M})=\rho\left(\mathcal{M}^{i}\right)$. If $\mathbf{m} \in \mathcal{M}$ then eventually $\mathbf{m} \in \mathcal{M}^{i}$ and so eventually $\rho\left(\mathcal{N}^{i}\right) \mid(\operatorname{supp} \mathbf{m}) \in \mathcal{M}$ and eventually these equal $\rho(\mathcal{M}) \mid(\operatorname{supp} \mathbf{m})$.

(d) Obvious from (b) and (c).

(e) A convergent sequence of bounded labels with a bounded limit has a flat limit by $(\mathrm{d})$.

(f) If $\mathcal{M}$ is flat then it is bounded and contains every element of the set $\Theta(\mathcal{M})$. The result then follows from (a), with $L=\mathbb{N}$, together with (e).

Recall that Supp $\mathcal{M}$ f-contains $L$ when $\mathcal{P}_{f} L \subset S$ upp $\mathcal{M}$, i.e. every finite subset of $L$ is the support of an element of $\mathcal{M}$, or, equivalently, if $\langle\chi(L)\rangle \subset \mathcal{M}$. By Proposition 2.5 if a label f-contains an infinite set then it is not of finite type and the converse holds if the label is bounded and not of finite type then it f-contains an infinite set.

Lemma 5.15. Let $L \subset \mathbb{N}$. A label $\mathcal{M}$ is flat over $L$ and Supp $\mathcal{M} f$-contains $L$ exactly when for any finite subset $F$ of $L, \rho(\mathcal{M}) \mid F \in \mathcal{M}$. In that case, $\{\chi(\ell): \ell \in L\}$ is an independent set in $\mathcal{M}$.

Proof: The first sentence is clear from the definitions. If $F$ is a finite subset of $L$ and $A \subset F$, then $\rho(\mathcal{M})|F=\rho(\mathcal{M})| A+\rho(\mathcal{M}) \mid(F \backslash A) \in \mathcal{M}$. So $\rho(\mathcal{M})|A \in \mathcal{M}-\rho(\mathcal{M})|(F \backslash A)$ and so $\{\chi(\ell): \ell \in A\} \subset \mathcal{M}-\rho(\mathcal{M}) \mid(F \backslash A)$. But if $\ell \in F \backslash A$ then $\chi(\ell) \notin \mathcal{M}-\rho(\mathcal{M}) \mid(F \backslash A)$. This means that $\{\chi(\ell): \ell \in L\}$ is an independent set.

Remark 5.16. It clearly suffices that $\rho(\mathcal{M}) \mid F_{k} \in \mathcal{M}$ for some increasing sequence $\left\{F_{k}\right\}$ of finite subsets with union $L$.

Proposition 5.17. Let $\mathcal{M}$ be a bounded label with $L=\operatorname{supp} \rho(\mathcal{M})$.

(a) If $\mathcal{M}$ is flat and f-contains $L$, then $\mathcal{M}$ is a strongly recurrent label.

(b) If $\mathcal{M}$ is a strongly recurrent label, then there exists an infinite set $L_{1} \subset L$ such that $\mathcal{M}$ is flat over $L_{1}$ and Supp $\mathcal{M} f$-contains $L_{1}$.

Proof: (a) In this case, $\mathcal{M}$ is a sublattice of $F I N(\mathbb{N})$ and so it is a strongly recurrent label by Proposition 2.22 (e). 
(b) Assume that inductively that we have defined $F_{k}=\left\{\ell_{1}, \ldots, \ell_{k}\right\}$ of distinct points of supp $\rho(\mathcal{M})$ such that $\rho(\mathcal{M}) \mid F_{k} \in \mathcal{M}$. Because $\mathcal{M}$ is strongly recurrent we can add, in $\mathcal{M}$ any element with support outside of the finite set $F\left(\rho(\mathcal{M}) \mid F_{k}\right)$. So for sufficiently large $\ell_{k+1}$ we have that $\rho(\mathcal{M}) \mid F_{k+1}=$ $\rho(\mathcal{M}) \mid F_{k}+\mathbf{r}(\mathcal{M})_{\ell_{k+1}} \chi\left(\ell_{k+1}\right) \in \mathcal{M}$. Let $F_{k+1}=F_{k} \cup\left\{\ell_{k+1}\right\}$. Then let $L_{1}=\bigcup_{k}\left\{F_{k}\right\}$.

Corollary 5.18. Assume that $\mathcal{M}$ is a bounded label not of finite type. If $\mathcal{M}$ is flat or strongly recurrent then it is non-tame.

Proof: If $\mathcal{M}$ is strongly recurrent then by Proposition 5.17 there exists an infinite subset $L_{1}$ of supp $\rho(\mathcal{M})$ such that $\mathcal{M}$ is flat over $L_{1}$ and Supp $\mathcal{M}$ f-contains $L_{1}$. By Proposition 2.5(b) any label not of finite type f-contains some infinite set $L_{1}$ and if $\mathcal{M}$ is flat then it is flat over $L_{1}$. By Lemma 5.15 $\left\{\chi(\ell): \ell \in L_{1}\right\}$ is an independent set in $\mathcal{M}$.

If we define for a bounded label $\mathcal{M}$

$$
\mathcal{F}(\mathcal{M}, L)=\{F: F \quad \text { is a finite subset of } L \text { and } \rho(\mathcal{M}) \mid F \in \mathcal{M}\},
$$

then $\mathcal{M}$ is flat over $L$ and $S$ upp $\mathcal{M}$ f-contains $L$ exactly when $\mathcal{F}(\mathcal{M}, L)=\mathcal{P}_{f} L$.

We conjecture that for every bounded $\mathcal{M}$ not of finite type there exists $\mathcal{N} \in \Theta(\mathcal{M})$ which is non-tame and so that $(X(\mathcal{M}), S)$ is not tame. Beyond the above corollary the best we can do is the following.

Proposition 5.19. Let $\mathcal{M}$ be a label not of finite type. If there exists $N \in \mathbb{N}$ such that $\rho(\mathcal{M}) \leq N$ then there exists $\mathcal{N} \in \Theta(\mathcal{M})$ which is non-tame.

Proof: If $K \in \mathbb{N}$ and $\rho(\mathcal{M}) \leq K$ then $\rho(\mathcal{N}) \leq K$ for all $\mathcal{N} \in[[\mathcal{N}]]$. By Proposition $2.20(\mathrm{~d})$ there is a positive recurrent label in $\Theta(\mathcal{M})$ and so we can assume that $\mathcal{M}$ itself is recurrent. By Proposition $2.22(\mathrm{~d})$ we can choose an infinite set $L \subset \operatorname{supp} \rho(\mathcal{M})$ such that $\left\{\chi(F): F \in \mathcal{P}_{f}(L)\right\}$ is a strongly recurrent set for $\mathcal{M}$. Consider $\mathcal{F}(\mathcal{M}, L)$.

Case (i): If there exists $\left\{F^{i}\right\}$ a strictly increasing sequence of elements of $\mathcal{F}(\mathcal{M}, L)$ with $L_{1}=\bigcup\left\{F^{i}\right\}$ then $\mathcal{F}\left(\mathcal{M}, L_{1}\right)=\mathcal{P}_{f} L_{1}$ and so $\mathcal{M}$ itself is non-tame by Lemma 5.15 .

Case (ii): If $F$ is a maximal element of $\mathcal{F}(\mathcal{M}, L)$ then $\rho(\mathcal{M}-\rho(\mathcal{M}) \mid F)_{\ell}=0$ for $\ell \in F$ and for $\ell \in L$ with

$$
\rho(\mathcal{M})_{\ell}>0 \quad \Longrightarrow \quad \rho(\mathcal{M})_{\ell}>\rho(\mathcal{M}-\rho(\mathcal{M}) \mid F)_{\ell}
$$

because for $\ell \in L \backslash F, \quad \rho(\mathcal{M})_{\ell} \notin \mathcal{M}-\rho(\mathcal{M}) \mid F$ by maximality of $F$. Let $\mathcal{M}_{1}=\mathcal{M}-\rho(\mathcal{M}) \mid F$ and $L_{1}=L \backslash F(\rho(\mathcal{M}) \mid F)$. We see that $\mathcal{M}_{1}$ is a recurrent 
element of $\Theta(\mathcal{M})$ with $\left\{\chi(F): F \in \mathcal{P}_{f}\left(L_{1}\right)\right\}$ a strongly recurrent set for $\mathcal{M}_{1}$. Furthermore, $\rho\left(\mathcal{M}_{1}\right) \leq K-1$ by (5.7). In particular, this cannot happen if $K=1$.

If Case (ii) occurs then we repeat the procedure with $\mathcal{M}$ and $L$ replaced by $\mathcal{M}_{1}$ and $L_{1}$. Eventually, we must terminate in a Case i situation and so at some $\mathcal{M}_{k} \in \Theta(\mathcal{M})$ which is non-tame.

Remark 5.20. Notice that Case (i) did not require that $\rho(\mathcal{M})$ is bounded by a constant. Furthermore, once we have the set $L$ associated with the strongly recurrent subset of $\mathcal{M}$ we can replace it by any infinite subset. In particular, if there is any infinite subset of $L$ on which $\rho(\mathcal{N})$ is bounded by a constant then the above argument will apply. Thus, the obstruction to proving the conjecture in general arises when $\operatorname{Lim} \rho(\mathcal{M})_{\ell}=\infty$ as $\ell \rightarrow \infty$ in $L$ and every element of $\mathcal{F}(\mathcal{M}, L)$ is contained in a maximal element of $\mathcal{F}(\mathcal{M}, L)$ and these conditions continue to hold as we replace $\mathcal{M}$ and $L$ by $\mathcal{M}-\rho(\mathcal{M}) \mid F, L_{1}$ for $F$ any maximal element of $\mathcal{F}(\mathcal{M}, L)$. Finally, we notice that if $\mathcal{F}(\mathcal{M}, L)$ contains sets of arbitrarily large cardinality then $\mathcal{M}$ is at least non-null by Lemma 5.15.

Corollary 5.21. Let $\mathcal{M}$ be a bounded label not of finite type.

(1) There is a label $\mathcal{N} \in[[\mathcal{M}]]$ (i.e. $\mathcal{N} \subset \mathcal{M}$ ) which is not of finite type and with $\rho(\mathcal{N})$ bounded by a constant. In particular, $\mathcal{N}$ is not tame.

(2) There is a label $\mathcal{N} \supset \mathcal{M}$ and with Supp $\mathcal{N}=$ Supp $\mathcal{M}$ which is flat, hence not tame.

Proof: (1). As $\mathcal{M}$ is not of finite type there is a strictly increasing sequence $\left\{\mathbf{m}_{i}\right\}_{i=1}^{\infty}$ of elements of $\mathcal{M}$. Let $\mathcal{N}=\left\langle\left\{\chi\left(\operatorname{supp} \mathbf{m}_{i}\right): i=1,2, \ldots\right\}\right\rangle$. Then clearly $\mathcal{N}$ is not of finite type and $\rho(\mathcal{N}) \leq 1$. The non-tameness follows from Proposition 5.19.

(2). Let $\mathcal{N}=\langle\{\rho(\mathcal{M}):(\operatorname{supp} \mathbf{m}): \mathbf{m} \in \mathcal{M}\}\rangle$. Clearly $\mathcal{N} \supset \mathcal{M}$ and is flat, hence not tame by Corollary 5.18 .

\section{Questions 5.22.}

(1) Is it true that for every label $\mathcal{M}$ not of finite type there exists $\mathcal{N} \in \Theta(\mathcal{M})$ which is non-tame (hence also so that $(X(\mathcal{M}), S)$ is not tame) ?

(2) Is there a label $\mathcal{M}$ not of finite type such that $X(\mathcal{M})$ is tame or even null?

Since a subsystem of a tame (or null) system is tame (resp. null), it follows that we could choose $\mathcal{M}$ in the latter case to be a recurrent label. A positive answer 
to this second question (in the null case) would thus yield an example of a null dynamical system with a recurrent transitive point which is not minimal. The question whether such a system exists is a long standing open question.

In a private conversation Tomasz Downarowicz asked us whether it is the case that every WAP system is null. Our next example shows that there are (a) non-null simple labels, hence topologically transitive WAP subshifts which are non-null; (b) non-tame labels of finite type, hence subshifts arising from finite type labels which are not tame.

Example 5.23. There are simple, finitary labels which are non-null. Accordingly, by Corollary 5.12, the corresponding subshifts are topologically transitive WAP subshifts which are non-null. Also, there are labels of finite type which are non-tame. Again the corresponding subshifts are topologically transitive and non-tame. Note that by Remark 5.11 these latter subshifts are not WAP.

(a) Partition $\mathbb{N}$ into disjoint sets $\left\{A_{n}: n \in \mathbb{N}\right\}$ with $\# A_{n}=n$. Define $\mathcal{M}_{n}$ by $\mathcal{M}_{n}=\left\langle\chi\left(A_{n}\right)\right\rangle$. By Lemma $5.15\left\{\chi(i): i \in A_{n}\right\}$ is an independent set for $\mathcal{M}_{n}$. Since $\left\{\mathcal{M}_{n}\right\}$ is a pairwise disjoint sequence of finite labels, $\mathcal{M}=\bigcup_{n}\left\{\mathcal{M}_{n}\right\}$ is a simple, finitary label which is clearly non-null.

(b) Partition $\mathbb{N}$ into two disjoint infinite sets $L, B$ and define a bijection $A \mapsto \ell_{A}$ from the set of finite subsets of $L$ onto $B$. Define $\mathcal{M}=\left\langle\left\{\chi\left(\ell_{A}\right)+\chi(i)\right.\right.$ : $i \in A, A$ a finite subset of $L\}\rangle$. Because it is size bounded, the label $\mathcal{M}$ is of finite type. Since $\mathcal{M}-\chi\left(\ell_{A}\right)=\langle\{\chi(i): i \in A\}\rangle$ it follows that $\{\chi(i): i \in L\}$ is an independent set for $\mathcal{M}$.

Remark 5.24. By Proposition 5.10 the label $\mathcal{M}$ of example (b) has $\Theta(\mathcal{M})$ uncountable. So this and Example 2.29 are labels $\mathcal{M}$ of finite type with $\Theta(\mathcal{M})$ uncountable. It follows that the corresponding subshifts $(X(\mathcal{M}), S)$ are LE but not HAE (see Remark 1.25).

\subsection{Gamow transformations.}

For $L \subset \mathbb{N}$ we let $F I N(L)=\{\mathbf{m} \in F I N(\mathbb{N}):$ supp $\mathbf{m} \subset L\}$ and $\mathcal{L} \mathcal{A B}(L)=\{\mathcal{M} \in \mathcal{L} \mathcal{A B}: \bigcup$ Supp $\mathcal{M} \subset L\}$. Clearly, $\mathcal{M} \in \mathcal{L} \mathcal{A B}(L)$ implies $[[\mathcal{M}]] \subset \mathcal{L} \mathcal{A B}(L)$. If $\mathcal{M} \notin \mathcal{L} \mathcal{A B}(L)$ then for some $N \in \mathbb{N} \mathcal{M} \cap \mathcal{B}_{N} \notin \mathcal{L A B}(L)$ 
and so $d\left(\mathcal{M}, \mathcal{M}_{1}\right)<2^{-N}$ implies $\mathcal{M}_{1} \notin \mathcal{L} \mathcal{A B}(L)$. Thus, $\mathcal{L} \mathcal{A B}(L)$ is a closed subset of $\mathcal{L} \mathcal{A B}$. For example, $\mathcal{L} \mathcal{A B}(\emptyset)=\{0, \emptyset\}$.

$F I N(L)$ is a submonoid of $F I N(\mathbb{N})$ and it acts on $\mathcal{L} \mathcal{A B}(L)$. Furthermore, if $\mathbf{r} \notin F I N(L)$ then $P_{\mathbf{r}}(\mathcal{M})=\emptyset$ for all $\mathcal{M} \in \mathcal{L} \mathcal{A B}(L)$. Hence, we can restrict attention to this action and for $\Phi$ any closed, invariant subset of $\mathcal{L} \mathcal{A B}(L)$, the enveloping semigroup $\mathcal{E}(\Phi)$ is the closure of $F I N(L)$ in $\Phi^{\Phi}$.

Let $\tau: L_{1} \rightarrow L_{2}$ be a bijection with $L_{1}, L_{2} \subset \mathbb{N}$. In honor of the book One, Two, Three,... Infinity we will refer to the following as the Gamow transformation induced by $\tau$. For an $\mathbb{N}$-vector $\mathbf{m}$ with supp $\mathbf{m} \subset L_{2}$ we let $\tau^{*} \mathbf{m}=\mathbf{m} \circ \tau$ so that supp $\tau^{*} \mathbf{m}=\tau^{-1}(\operatorname{supp} \mathbf{m}) \subset L_{1}$. Thus, $\tau^{*}: F I N\left(L_{2}\right) \rightarrow F I N\left(L_{1}\right)$ is a monoid isomorphism which also preserves the lattice properties.

For $\mathcal{M} \in \mathcal{L} \mathcal{A B}\left(L_{2}\right)$ we let $\tau^{*} \mathcal{M}=\left\{\tau^{*} \mathbf{m}: \mathbf{m} \in \mathcal{M}\right\}$ and for $\Phi \subset \mathcal{L} \mathcal{A B}\left(L_{2}\right)$ we will let $\tau^{*} \Phi=\left\{\tau^{*} \mathcal{N}: \mathcal{N} \in \Phi\right\}$. Thus, $\tau^{*}$ is a bijection from $\mathcal{L} \mathcal{A B}\left(L_{2}\right)$ to $\mathcal{L} \mathcal{A B}\left(L_{1}\right)$ with inverse $\left(\tau^{-1}\right)^{*}$.

Given $\ell \in \mathbb{N}$, let $\ell^{\prime}=\max \tau\left([1, \ell] \cap L_{1}\right)$. It follows from the definition (2.6) of the metric on $\mathcal{L} \mathcal{A B}$ that $d\left(\mathcal{M}_{1}, \mathcal{M}_{2}\right) \leq 2^{-\ell^{\prime}}$ implies $d\left(\tau^{*}\left(\mathcal{M}_{1}\right), \tau^{*}\left(\mathcal{M}_{2}\right)\right) \leq 2^{-\ell}$. Thus, the $\tau^{*}$ is uniformly continuous on $\mathcal{L} \mathcal{A B}\left(L_{2}\right)$ and so is a homeomorphism from $\mathcal{L} \mathcal{A B}\left(L_{2}\right)$ onto $\mathcal{L} \mathcal{A} \mathcal{B}\left(L_{1}\right)$.

Clearly, $\tau^{*}$ preserves all label operations, e.g. $\tau^{*}(\mathcal{M}-\mathbf{r})=\tau^{*} \mathcal{M}-\tau^{*} \mathbf{r}$ for $\mathcal{M} \in \mathcal{L} \mathcal{A} \mathcal{B}\left(L_{2}\right)$ and $\mathbf{r} \in F I N\left(L_{2}\right)$. Thus, $\tau^{*}$ is an action isomorphism relating the $F I N\left(L_{2}\right)$ action on $\mathcal{L} \mathcal{A B}\left(L_{2}\right)$ to the $F I N\left(L_{1}\right)$ action on $\mathcal{L} \mathcal{A} \mathcal{B}\left(L_{1}\right)$. Hence, it induces an Ellis semigroup isomorphism from $\mathcal{E}(\Phi)$ to $\mathcal{E}\left(\tau^{*}(\Phi)\right)$ where $\Phi$ is a compact, invariant subset of $\mathcal{L} \mathcal{A B}\left(L_{2}\right)$. Also $\Theta\left(\tau^{*} \mathcal{M}\right)=\tau^{*} \Theta(\mathcal{M})$ for $\mathcal{M} \in \mathcal{L} \mathcal{A B}\left(L_{2}\right)$.

Thus, all of the label properties are preserved by $\tau^{*}$. For example, $\tau^{*} \mathcal{M}$ is bounded, of finite type, finitary, simple, semi-simple, WAP, recurrent or strongly recurrent iff $\mathcal{M}$ satisfies the corresponding property. A label $\mathcal{F}$ is an external element for $\mathcal{E}(\Theta(\mathcal{M}))$ iff $\tau^{*} \mathcal{F}$ is an external element for $\mathcal{E}\left(\Theta\left(\tau^{*} \mathcal{M}\right)\right.$.

On the other hand, the subshifts $\left(X\left(\tau^{*} \mathcal{M}\right), S\right)$ and $(X(\mathcal{M}), S)$ are only analogous. That is, they have similar properties but are not usually isomorphic. For the finite type case, see Corollary 3.37.

Let $S_{\infty}$ denote the group of all permutations on $\mathbb{N}$. On $S_{\infty}$ we define an ultrametric by

$$
d\left(\tau_{1}, \tau_{2}\right)=\inf \left\{2^{-\ell}: \ell \in \mathbb{Z}_{+} \text {and } \tau_{1}\left|[1, \ell]=\tau_{2}\right|[1, \ell]\right\} .
$$

Clearly, for any $\gamma \in S_{\infty}, d\left(\gamma \circ \tau_{1}, \gamma \circ \tau_{2}\right)=d\left(\tau_{1}, \tau_{2}\right)$. If $\gamma([1, \ell]) \subset\left[1, \ell_{\gamma}\right]$ then $\tau_{1}\left|\left[1, \ell_{\gamma}\right]=\tau_{2}\right|\left[1, \ell_{\gamma}\right]$ implies $\tau_{1} \circ \gamma\left|[1, \ell]=\tau_{2} \circ \gamma\right|[1, \ell]$ and $\gamma_{1}\left|\left[1, \ell_{\gamma}\right]=\gamma\right|\left[1, \ell_{\gamma}\right]$ then $\gamma_{1}^{-1}\left|[1, \ell]=\gamma^{-1}\right|[1, \ell]$. It follows that $S_{\infty}$ is a topological group with left invariant ultrametric $d$. Furthermore, the equivalent metric $\bar{d}$ given by $\bar{d}\left(\tau_{1}, \tau_{2}\right)=\max \left(d\left(\tau_{1}, \tau_{2}\right), d\left(\tau_{1}^{-1}, \tau_{2}^{-1}\right)\right)$ is complete. Finally, the set of permutations $S_{\text {fin }}$ consisting of permutations are the identity on the complement of a 
finite set, is a countable dense subgroup of $S_{\infty}$. Thus, $S_{\infty}$ is a Polish group, which is clearly perfect.

Furthermore, if $\mathcal{M}$ and $\mathcal{M}_{1}$ are labels with $\mathcal{M} \cap \mathcal{B}_{\ell_{\gamma}}=\mathcal{M}_{1} \cap \mathcal{B}_{\ell_{\gamma}}$ and $\gamma_{1} \mid\left[1, \ell_{\gamma}\right]=$ $\gamma \mid\left[1, \ell_{\gamma}\right]$ then $\gamma^{*} \mathcal{M} \cap \mathcal{N}_{\ell}=\gamma_{1}^{*} \mathcal{M}_{1} \cap \mathcal{N}_{\ell}$. This implies that the action $S_{\infty} \times \mathcal{L} \mathcal{A B} \rightarrow$ $\mathcal{L} \mathcal{A B}$ given by $(\tau, \mathcal{M}) \rightarrow\left(\tau^{-1}\right)^{*} \mathcal{M}$ is a continuous action. The empty label $\emptyset$ is an isolated fixed point for the action. Let $\mathcal{L} \mathcal{A} \mathcal{B}_{+}$denote the perfect set of nonempty labels. We show that this action is topologically transitive on $\mathcal{L} \mathcal{A B}+$ by constructing explicitly a transitive point.

Example 5.25. Let $\Xi$ be the countable set of all pairs $\left(\mathcal{N}_{\xi}, \ell_{\xi}\right)$ with $\mathcal{N}_{\xi}$ a finite label such that $\bigcup \operatorname{Supp} \mathcal{N}_{\xi} \subset\left[1, \ell_{\xi}\right]$. Partition $\mathbb{N}$ by disjoint intervals indexed by $\Xi$ such that $I_{\xi}$ has length $\ell_{\xi}$. Let $\tau_{\xi}: I_{\xi} \rightarrow\left[1, \ell_{\xi}\right]$ be the increasing linear bijection and let $\mathcal{M}_{\xi}=\tau_{\xi}^{*} \mathcal{N}_{\xi}$ so that $\bigcup$ Supp $\mathcal{M}_{\xi} \subset I_{\xi}$. Let $\mathcal{M}_{\text {trans }}=\bigcup_{\xi} \mathcal{M}_{\xi}$. By Corollary $4.32 \mathcal{M}_{\text {trans }}$ is finitary and simple and so is of finite type. On the other hand, given any nonempty label $\mathcal{M}$ and any $N \in \mathbb{N}$ there exists $\xi \in \Xi$ such that $\left(\mathcal{N}_{\xi}, \ell_{\xi}\right)=\left(\mathcal{M} \cap B_{N}, N\right)$. It follows that if $\gamma \in S_{\text {fin }}$ with $\gamma=\tau_{\xi}$ on $I_{\xi}$ then $\left(\gamma^{-1}\right)^{*} \mathcal{M}_{\text {trans }} \cap B_{N}=\mathcal{M} \cap B_{N}$. Thus, $\mathcal{M}_{\text {trans }}$ is a transitive point for the action of $S_{\infty}$ on $\mathcal{L} \mathcal{A B} \mathcal{B}_{+}$.

Because $\mathcal{L} \mathcal{A B} \mathcal{B}_{+}$is a Cantor set and $S_{\infty}$ is a group, the set TRANS of $S_{\infty}$ transitive points is a dense $G_{\delta}$ subset of $\mathcal{L} \mathcal{A B} \mathcal{B}_{+}$. By Proposition 2.35 the set $R E C U R$ of recurrent labels is a dense $G_{\delta}$ subset of $\mathcal{L A B}$. Hence,

$T R A N S \cap R E C U R$ is a dense $G_{\delta}$ subset of $\mathcal{L} \mathcal{A} \mathcal{B}_{+}$. The transitive point $\mathcal{M}_{\text {trans }}$ is of finite type and so is not recurrent. On the other hand, the set of flat labels is a proper, closed $S_{\infty}$ invariant subset which contains recurrent labels (see Proposition 5.17 (a)) which are thus not transitive with respect to the $S_{\infty}$ action.

For background regarding our next question we refer the reader to the works [33] and [26].

Question 5.26. Does there exist a label $\mathcal{M}$ such that its $S_{\infty}$ orbit is residual, or are all the orbits meager? If such a residual orbit exists then it would be unique and would meet and so be contained in TRANS $\cap R E C U R$. It is well known that the adjoint action of $S_{\infty}$ on itself does have a dense $G_{\delta}$ orbit (see e.g. [26]).

\subsection{Ordinal constructions.}

Assume that $(X, T)$ is a cascade with $X$ metrizable. For $A \subset X$ we defined $z_{L I M}(A)=\overline{R_{T}(A)}$, the closure of the set of all positive or negative limit points 
of orbits of points of $A$, and $R_{T}^{*}(A)=\left\{x: R_{T}(x) \subset A\right\}$. We defined in (1.3) the LIM descending transfinite sequence of closed sets by

$$
z_{0}(X)=X, \quad z_{\alpha+1}(X)=z\left(z_{\alpha}(X)\right), \quad z_{\beta}=\bigcap_{\alpha<\beta} z_{\alpha}(X)
$$

for $\beta$ a limit ordinal. This sequence stabilizes at the height being the first ordinal $\beta$ such that $z_{\beta}$ is the Birkhoff center, i.e. the closure of the set of recurrent points.

Assume that $(X, T)$ is a cascade with $Y$ an orbit-closed subset of $X$. We defined in (1.6) the increasing transfinite sequence of orbit-closed sets, by

$$
z_{0}^{*}(Y)=Y, \quad z_{\alpha+1}^{*}(Y)=R_{T}^{*}\left(z_{\alpha}^{*}(Y)\right), \quad z_{\beta}^{*}(Y)=\bigcup_{\alpha<\beta} z_{\alpha}^{*}(Y)
$$

for $\beta$ a limit ordinal. This sequence stabilizes at the minimum L-determined set in $X$ which contains $Y$. If $(X, T)$ is a metrizable, CT-WAP system with the fixed point an isolated invariant set, then $X$ is the only L-determined set by Corollary 1.42 and the height* is the first ordinal $\beta$ such that with $Y=\{e\}$ $z_{\beta}^{*}(e)=X$. If the system is topologically transitive with a transitive point $x^{*}$ then the height* is the first ordinal $\beta$ such that $x^{*} \in z_{\beta}^{*}(e)$.

Now we define the label versions of these constructions.

For a label $\mathcal{M}$ define the label

$$
z_{L A B}(\mathcal{M})=\mathcal{M} \backslash \max \mathcal{M}=\{\mathbf{m}: \mathbf{m}+\mathbf{r} \in \mathcal{M} \text { for some } \mathbf{r}>\mathbf{0}\}
$$

Thus $\mathbf{m} \in z_{L A B}(\mathcal{M})$ iff there exists $\mathbf{m}_{1} \in \mathcal{M}$ with $\mathbf{m}<\mathbf{m}_{1}$.

Lemma 5.27. Let $\mathcal{M}, \mathcal{M}_{1}$ be labels.

(a) $z_{L A B}(\mathcal{M}) \subset \mathcal{M}$ and the inclusion is proper if $\mathcal{M}$ is nonempty and of finite type.

(b) $\mathcal{M}_{1} \subset \mathcal{M}$ implies $z_{L A B}\left(\mathcal{M}_{1}\right) \subset z_{L A B}(\mathcal{M})$.

(c) If $\left\{\mathcal{M}_{i}\right\}$ is a collection of labels then $\bigcup_{i} z_{L A B}\left(\mathcal{M}_{i}\right)=z_{L A B}\left(\bigcup_{i} \mathcal{M}_{i}\right)$.

(d) $z_{L A B}(\mathcal{M})=\emptyset$ iff $\mathcal{M}=0$ or $\emptyset$.

Proof: (a) and (b) are obvious and so $\bigcup_{i} z_{L A B}\left(\mathcal{M}_{i}\right) \subset z_{L A B}\left(\bigcup_{i} \mathcal{M}_{i}\right)$. If $\mathbf{m} \in z_{L A B}\left(\bigcup_{i} \mathcal{M}_{i}\right)$ then there exists $\mathbf{m}_{1}>\mathbf{m}$ with $\mathbf{m}_{1} \in \bigcup_{i} \mathcal{M}_{i}$. So $\mathbf{m}_{1} \in \mathcal{M}_{i}$ for some $i$ and this implies $\mathbf{m} \in z_{L A B}\left(\mathcal{M}_{i}\right)$, proving (c).

(d): If $\mathcal{M}$ is positive then $\mathbf{0} \in z_{L A B}(\mathcal{M})$. On the other hand, if $\mathcal{M}=0$ or $\emptyset$ then $z_{L A B}(\mathcal{M})=\emptyset$. 
Define a descending transfinite sequence of labels by

$$
\begin{gathered}
z_{L A B, 0}(\mathcal{M})=\mathcal{M} \\
z_{L A B, \alpha+1}(\mathcal{M})=z_{L A B}\left(z_{L A B, \alpha}(\mathcal{M})\right), \\
z_{L A B, \beta}(\mathcal{M})=\bigcap_{\alpha<\beta}\left\{z_{L A B, \alpha}(X)\right\} \quad \text { for } \beta \text { a limit ordinal. }
\end{gathered}
$$

The sequence stabilizes at $\beta$ when $z_{L A B, \beta}(\mathcal{M})=z_{L A B, \beta+1}(\mathcal{M})$ in which case $z_{L A B, \alpha}(\mathcal{M})=z_{L A B, \beta}(\mathcal{M})$ for all $\alpha \geq \beta$. So $\emptyset$ stabilizes at 0 and if $\mathcal{M}$ is nonempty and of finite type then the sequence stabilizes at $\beta+1$ where $\beta$ is the first ordinal for which $z_{L A B, \beta}=0$.

If $\Phi$ is a closed, bounded, invariant set of labels define $z_{L A B}(\Phi)$ to be the closure of $\bigcup\left\{P_{\mathbf{r}}(\Phi): \mathbf{r}>\mathbf{0}\right\}$, a closed, invariant subset of $\Phi$. Thus, $z_{L A B}(\Theta(\mathcal{M}))=\Theta^{\prime}(\mathcal{M})$.

Define the nonincreasing transfinite sequence of closed, bounded subsets of $\mathcal{L A B}$ by

$$
\begin{gathered}
z_{L A B, 0}(\Phi)=\Phi \\
z_{L A B, \alpha+1}(\Phi)=z_{L A B}\left(z_{L A B, \alpha}(\Phi)\right) \\
z_{L A B, \beta}(\Phi)=\bigcap_{\alpha<\beta}\left\{z_{L A B, \alpha}(\Phi)\right\} \quad \text { for } \beta \text { a limit ordinal. }
\end{gathered}
$$

Theorem 5.28. Let $\Phi$ be a closed, $F I N(\mathbb{N})$ invariant subset of $\mathcal{L} \mathcal{A B}$ and $\mathcal{M}=\bigcup \Phi$, e.g. $\Phi=\Theta(\mathcal{N})$ for any label $\mathcal{N}$. Then $z_{L A B, \alpha}(\mathcal{M})=\bigcup z_{L A B, \alpha}(\Phi)$ for every countable ordinal $\alpha$. That is, $\mathbf{m} \in z_{L A B, \alpha}(\mathcal{M})$ iff there exists $\mathcal{N} \in$ $z_{L A B, \alpha}(\Phi)$ such that $\mathbf{m} \in \mathcal{N}$.

Proof: We use transfinite induction. Both procedures stabilize at a countable ordinal and so we need only consider countable ordinals.

Since $\mathcal{M}=\bigcup \Phi$ the result is true for $\alpha=0$. Notice that for any label $\mathcal{M}=\bigcup \Theta(\mathcal{M})$.

If $\mathbf{m} \in z_{L A B, \alpha+1}(\mathcal{M})=z_{L A B}\left(z_{L A B, \alpha}(\mathcal{M})\right)$, then there exists $\mathbf{r}>\mathbf{0}$ such that $\mathbf{m}+\mathbf{r} \in z_{L A B, \alpha}(\mathcal{M})$. By induction hypothesis, there exists $\mathcal{N} \in z_{L A B, \alpha}(\Phi)$ such that $\mathbf{m}+\mathbf{r} \in \mathcal{N}$ and so $\mathbf{m} \in \mathcal{N}-\mathbf{r} \in z_{L A B, \alpha+1}(\Phi)$.

Conversely, if $\mathcal{N} \in z_{L A B, \alpha+1}(\Theta(\mathcal{M}))=z_{L A B}\left(z_{L A B, \alpha}(\Phi)\right)$ then there exists sequences $\mathcal{N}^{i} \in z_{L A B, \alpha}(\Phi)$ and $\mathbf{r}^{i}>0$ such that $\mathcal{N}=L I M\left\{\mathcal{N}^{i}-\mathbf{r}^{i}\right\}$. By induction hypothesis $\bigcup\left\{\mathcal{N}^{i}\right\} \subset z_{L A B, \alpha}(\mathcal{M})$. If $\mathbf{m} \in \mathcal{N}$ then eventually $\mathbf{m} \in$ $\mathcal{N}^{i}-\mathbf{r}^{i}$ and so $\mathbf{m} \in z_{L A B, \alpha+1}(\mathcal{M})$.

Now let $\beta$ be a limit ordinal. If $\mathbf{m} \in \mathcal{N} \in z_{L A B, \beta}(\Phi)$ then by definition $\mathcal{N} \in z_{L A B, \alpha}(\Phi)$ for all $\alpha<\beta$. So by induction hypothesis, $\mathbf{m} \in z_{L A B, \alpha}(\mathcal{M})$ for all $\alpha<\beta$ and hence $\mathbf{m} \in z_{L A B, \beta}(\mathcal{M})$.

Conversely, if $\mathbf{m} \in z_{L A B, \beta}(\mathcal{M})$ and so in $z_{L A B, \alpha}(\mathcal{M})$ for all $\alpha<\beta$ we let $\left\{\alpha^{i}\right\}$ be an increasing sequence of ordinals converging to $\beta$. By induction hypothesis 
there exists $\mathcal{N}^{i} \in z_{L A B, \alpha^{i}}(\Phi)$ such that $\mathbf{m} \in \mathcal{N}^{i}$. Since $\left\{\alpha^{i}\right\}$ is increasing $\mathcal{N}^{i} \in z_{L A B, \alpha^{j}}(\mathcal{N})$ for all $j<i$. Let $\left\{\mathcal{N}^{i^{\prime}}\right\}$ be a convergent subsequence with limit $\mathcal{N}$. Since $\mathbf{m} \in \mathcal{N}^{i}$ for all $i, \mathbf{m} \in \mathcal{N}$. For every $\alpha<\beta$ there exists $\alpha^{j}>\alpha$. For $i \geq j$ the sequence $\mathcal{N}^{i} \in z_{L A B, \alpha^{j}}(\Phi) \subset z_{L A B, \alpha}(\Phi)$ and so the limit of the subsequence $\mathcal{N}$ is in the closed set $z_{L A B, \alpha}(\Phi)$. Since this is true for all $\alpha<\beta$, $\mathcal{N} \in z_{L A B, \beta}(\Phi)$.

It follows that the sequences stabilize at the same countable ordinal. If $\mathcal{M}=\emptyset$ then $\Theta(\mathcal{M})=\{\emptyset\}$ and the sequence stabilizes at 0 . If $\mathcal{M}$ is nonempty and of finite type then the sequence stabilizes at $\beta+1$ where $\beta$ is the ordinal with $z_{L A B, \beta}(\mathcal{M})=0$ and $z_{L A B, \beta}(\Theta(\mathcal{M}))=\{0, \emptyset\}$. In this nonempty finite type case, we call $\beta+1$ the height of $\Theta(\mathcal{M})$.

Corollary 5.29. Assume that $\mathcal{M}$ is a label of finite type and that $\Phi$ is a nonempty, closed, FIN $(\mathbb{N})$ subset of $[[\mathcal{M}]]$. If $\Phi=z_{L A B}(\Phi)$ then $\Phi=\{\emptyset\}$.

Proof: If $\Phi=z_{L A B}(\Phi)$ then the transfinite sequence $\left\{z_{L A B, \alpha}(\Phi)\right\}$ stabilizes at $\alpha=0$. If $\mathcal{N}=\bigcup \Phi$ then since $\mathcal{N} \subset \mathcal{M}$ it is of finite type and so the sequence $\left\{z_{L A B \alpha}(\mathcal{N})\right\}$ stabilizes at $\alpha=0$ only when $\mathcal{N}=\emptyset$. But by Theorem 5.28 these two sequences stabilize at the same level.

For $Y$ a subset of $\{0,1\}^{\mathbb{Z}}$ Definition 3.31 says

$$
\Phi(Y)=\{\mathcal{N}: x[\mathcal{N}] \in Y\} \quad \text { and } \quad \Phi_{+}(Y)=\left\{\mathcal{N}: x_{+}[\mathcal{N}] \in Y\right\} .
$$

Theorem 5.30. Assume that $\mathcal{M}$ is a label of finite type. For every countable ordinal $\alpha$, every closed, invariant $Y \subset X(\mathcal{M})$ and every closed, invariant $Y_{+} \subset X_{+}(\mathcal{M})$

$$
\begin{array}{ccc}
\Phi\left(z_{L I M, \alpha}(Y)\right) & = & z_{L A B, \alpha}(\Phi(Y)), \\
\Phi_{+}\left(z_{L I M, \alpha}\left(Y_{+}\right)\right) & = & z_{L A B, \alpha}\left(\Phi_{+}\left(Y_{+}\right)\right) .
\end{array}
$$

Proof: The equation is clear for $\alpha=0$.

Since $\Phi(Y)$ is the preimage of $Y$ with respect to the continuous map $x[\cdot]$ it follows that $\Phi\left(z_{L I M}(Y)\right)$ is a closed invariant set containing $P_{\mathbf{r}} \mathcal{N}$ whenever $x[\mathcal{N}] \in Y$ and $\mathbf{r}>\mathbf{0}$ by Theorem 3.32(a) and the Remark after Theorem 4.15 which implies that $p_{\mathbf{r}}=q\left(P_{\mathbf{r}}\right) \in A(Y, S)$. Hence, $z_{L A B}(\Phi(Y)) \subset \Phi\left(z_{L I M}(Y)\right)$.

On the other hand, Theorem 3.34 (b) implies that $z_{L A B}(\Phi(Y))=\Phi(\tilde{Y})$ for a closed, invariant subspace $\tilde{Y}$ of $X(\mathcal{M})$. If $\mathbf{r}>0$ and $y \in Y$ then $y=S^{k}(x[\mathcal{N}])$ with $k \in \mathbb{Z}$ and $\mathcal{N} \in \Phi(Y)$. So $p_{\mathbf{r}} y=S^{k}\left(x\left[P_{\mathbf{r}} \mathcal{N}\right]\right)$. Since $P_{\mathbf{r}} \mathcal{N} \in z_{L A B}(\Phi(Y))$, it follows that $p_{\mathbf{r}} y \in \tilde{Y}$. Hence, $z_{L I M}(Y) \subset \tilde{Y}$ and so $\Phi\left(z_{L I M}(Y)\right) \subset \Phi(\tilde{Y})=$ $z_{L A B}(\Phi(Y))$. 
This proves equation (5.14) with $\alpha=1$ and so assuming the result for an ordinal $\alpha$ it follows for $\alpha+1$.

For a limit ordinal, $\beta$ we use the fact that $\Phi(\cdot)$ commutes with intersection and so by the induction hypothesis

$$
\begin{aligned}
\Phi\left(z_{L I M, \beta}(Y)\right) & =\Phi\left(\bigcap_{\alpha<\beta} z_{L I M, \alpha}(Y)\right)=\bigcap_{\alpha<\beta} \Phi\left(z_{L I M, \alpha}(Y)\right) \\
& =\bigcap_{\alpha<\beta} z_{L A B, \alpha}(\Phi(Y))=z_{L A B, \beta}(\Phi(Y)) .
\end{aligned}
$$

This completes the induction.

We will say that $\Phi \subset \mathcal{L} \mathcal{A B}$ is $\Theta$ invariant when it is nonempty orbit-closed with respect to the $F I N(\mathbb{N})$ action. That is, $\mathcal{M} \in \Phi$ implies $\Theta(\mathcal{M}) \subset \Phi$. If $\Phi$ is closed then it is invariant iff it is $\Theta$ invariant. $\Theta$ invariance always implies invariance but is usually a stronger condition since $\left\{P_{\mathbf{r}}(\mathcal{M})\right\}$ is usually a proper subset of $\Theta(\mathcal{M})$.

Let $\mathcal{M}$ be a label of finite type. For a $\Theta$ invariant $\Phi \subset \Theta(\mathcal{M})$, we define $z_{\mathcal{M}}^{*}(\Phi)=\left\{\mathcal{N} \in \Theta(\mathcal{M}): \Theta^{\prime}(\mathcal{N}) \subset \Phi\right\}$. Equivalently, $\mathcal{N} \in z_{\mathcal{N}}^{*}(\Phi)$ iff $Q(\mathcal{N}) \in \Phi$ for all $Q \in \mathcal{A}(\Theta(\mathcal{M}))=\mathcal{E}(\Theta(\mathcal{M})) \backslash\left\{i d_{\Theta(\mathcal{N})}\right\}$. For example, $z_{\mathcal{M}}^{*}(\{\emptyset\})=\{\emptyset, 0\}=$ $[[0]]=\Theta(0)$.

Starting with a $\Theta$ invariant $\Phi \subset \Theta(\mathcal{M})$, define the nondecreasing transfinite sequence of $\Theta$ invariant subsets of $\Theta(\mathcal{M})$ by

$$
\begin{gathered}
z_{\mathcal{M}, 0}^{*}(\Phi)=\Phi \\
z_{\mathcal{M}, \alpha+1}^{*}(\Phi)=z_{\mathcal{M}}^{*}\left(z_{\mathcal{M}, \alpha}^{*}(\Phi)\right) \\
z_{\mathcal{M}, \beta}^{*}(\Phi)=\bigcup_{\alpha<\beta}\left\{z_{\mathcal{M}, \alpha}^{*}(\Phi)\right\} \quad \text { for } \beta \text { a limit ordinal. }
\end{gathered}
$$

Recall that for a dynamical system $(X, T), Y \subset X$ is called orbit-closed when $x \in Y$ implies $\overline{O_{T}(x)} \subset Y$. For $\mathcal{M}$ of finite type it is easy to adjust the proof of Corollary 3.43 to show that $Y \subset X(\mathcal{M})$ is orbit-closed iff $\Phi(Y)$ is $\Theta$ closed and $Y_{+} \subset X_{+}(\mathcal{M})$ is orbit-closed iff $\Phi_{+}\left(Y_{+}\right)$is $\Theta$ closed.

Theorem 5.31. Assume that $\mathcal{M}$ is a label of finite type, that $Y$ is an orbit closed subset of $X(\mathcal{M})$ and that $Y_{+}$is an orbit closed subset of $X_{+}(\mathcal{M})$. For every countable ordinal $\alpha$,

$$
\begin{aligned}
\Phi\left(z_{\alpha}^{*}(Y)\right) & =z_{\mathcal{M}, \alpha}^{*}(\Phi(Y)), \\
\Phi_{+}\left(z^{*} \alpha\left(Y_{+}\right)\right) & =z_{\mathcal{M}, \alpha}^{*}\left(\Phi_{+}\left(Y_{+}\right)\right),
\end{aligned}
$$

Proof: This is obvious for $\alpha=0$.

A point $x \in R_{S}^{*}(Y)$ iff $q x \in Y$ for all $q \in A(X(\mathcal{M}), S)$. Since $Y$ is $S$ invariant, Theorem 4.15, and the remark thereafter, imply that this is true 
iff $\bar{D}(Q) x \in Y$ for all $Q \in \mathcal{A}(\Theta(\mathcal{M}))$. Hence, $x[\mathcal{N}] \in R_{S}^{*}(Y)$ iff $x[Q(\mathcal{N})] \in Y$ for all $Q \in \mathcal{A}(\Theta(\mathcal{M}))$, i.e. iff $Q(\mathcal{N}) \in \Phi(Y)$ for all $Q \in \mathcal{A}(\Theta(\mathcal{M}))$ and so iff $\mathcal{N} \in z_{\mathcal{M}}^{*}(\Phi(Y))$. This proves equation (5.17) for $\alpha=1$ and so inductively for any $\alpha+1$.

Since $\Phi$ is the preimage operator with respect to the map $x[\cdot]$, it commutes with union. So the equation for a limit ordinal $\beta$ follows because it is assumed, inductively, to hold for all $\alpha<\beta$.

The proof for $Y_{+}$is completely similar.

The constructions of (5.12), (5.13) and (5.16) are label constructions and so they commute with Gamow transformations. We can use Gamow transformations to assure that a countable number of labels all occur with supports on disjoint sets. Let $\tau_{0}: \mathbb{N} \rightarrow \mathbb{N} \times \mathbb{N}$ be a bijection and let $L_{i}=\tau_{0}^{-1}(\mathbb{N} \times\{i\})$ for $i \in \mathbb{N}$. Define $\tau_{i}: L_{i} \rightarrow \mathbb{N}$ to be the bijection $\tau_{i}=\pi_{1} \circ \tau_{0}$ where $\pi_{1}: \mathbb{N} \times \mathbb{N} \rightarrow \mathbb{N}$ is the first coordinate projection. Given a sequence $\left\{\mathcal{M}^{i}\right\}$ of labels, the label $\left\{\tau_{i}^{*} \mathcal{M}^{i}\right\}$ is Gamow equivalent to $\mathcal{M}^{i}$ and $\bigcup$ Supp $\mathcal{M}^{i} \subset L_{i}$.

For the following two propositions we apply Theorems 4.25 and 4.31 together with their proofs. Note that $[[0]]=\{\emptyset, 0\}$.

Proposition 5.32. Assume $\mathcal{N}$ and $\mathcal{M}$ are positive disjoint labels with $\mathcal{N}$ finite and $\mathcal{M}$ of finite type.

(a) If $\Psi \subset[[\mathcal{N}]]$ and $\Phi \subset[[\mathcal{M}]]$ are closed and invariant then

$$
z_{L A B}(\Psi \oplus \Phi)=z_{L A B}(\Psi) \oplus \Phi \cup \Psi \oplus z_{L A B}(\Phi) .
$$

And for every limit ordinal $\alpha$

$$
z_{L A B, \alpha}(\Theta(\mathcal{N} \oplus \mathcal{M}))=\{\mathcal{N}\} \oplus z_{L A B, \alpha}(\Theta(\mathcal{M})) .
$$

For $\alpha=0$ or a limit ordinal and $k \in \mathbb{N}$

$$
z_{L A B, \alpha+k}\left(\Theta\left(\mathcal{M}_{1} \oplus \mathcal{M}_{2}\right)\right)=\bigcup_{r=0}^{k} z_{L A B, \alpha+r}(\Theta(\mathcal{N})) \oplus z_{L A B, \alpha+k-r}(\Theta(\mathcal{M}))
$$

(b) For all $k \in \mathbb{N}$

$$
z_{\mathcal{N} \oplus \mathcal{M}, k}^{*}([[0]])=\bigcup_{r=0}^{k} z_{\mathcal{N}, r}^{*}([[0]]) \oplus z_{\mathcal{N}, k-r}^{*}([[0]])
$$

For every limit ordinal $\alpha$ and $k \in \mathbb{Z}_{+}$

$$
\left.z_{\mathcal{N} \oplus \mathcal{M}, \alpha+k}^{*}([[0]])=\Theta(\mathcal{N}) \oplus z_{\mathcal{M}, \alpha+r}^{*}([[0]])\right) .
$$

Proof: If $\mathbf{r}>0$ with $\mathbf{r} \in \mathcal{M}_{1} \oplus \mathcal{M}_{2}$ then $\mathbf{r}=\mathbf{r}_{1}+\mathbf{r}_{2}$ with either $\mathbf{r}_{1}>0$ or $\mathbf{r}_{2}>0$. 
It therefore follows that for $\Phi_{1} \subset \Theta\left(\mathcal{M}_{1}\right)$ and $\Phi_{2} \subset \Theta\left(\mathcal{M}_{2}\right)$ closed invariant subspaces,

$$
z_{L A B}\left(\Phi_{1} \oplus \Phi_{2}\right)=z_{L A B}(\Psi) \oplus \Phi \cup \Psi \oplus z_{L A B}(\Phi) .
$$

Then (5.20) follows by induction on $k$ since the operator $z_{L A B}$ commutes with union. For (5.19) we use induction on the limit ordinals together with 0 starting with $\alpha=0$. Assume the result for all $\beta<\alpha$. Since $\mathcal{N}$ is positive and finite, there exists a unique $r_{0} \in \mathbb{N}$ such that where $z_{L A B, r_{0}}(\Theta(\mathcal{N}))=[[0]]$. From (5.20) we have

$$
\begin{gathered}
\{\mathcal{N}\} \oplus z_{L A B, \beta+k}(\Theta(\mathcal{M})) \subset z_{L A B, \beta+k}(\Theta(\mathcal{N} \oplus \mathcal{M})) \\
\subset\{\mathcal{N}\} \oplus z_{L A B, \beta+k-r_{0}}(\Theta(\mathcal{M}))
\end{gathered}
$$

Intersecting we obtain (5.19) for $\alpha=\beta+\omega$. Otherwise, $\alpha$ is an increasing limit of limit ordinals and the result follow from the induction hypothesis by intersecting.

For (5.21) assume that $\Phi \subset \Theta(\mathcal{N})$ and $\Psi \subset \Theta(\mathcal{M})$ are $\Theta$ invariant. By Theorem 4.25 the labels $\Theta(\mathcal{N} \oplus \mathcal{M})$ are of the form $\mathcal{N}_{1} \oplus \mathcal{M}_{1}$ with $\mathcal{N}_{1} \in \Theta(\mathcal{N})$ and $\mathcal{M}_{1} \in \Theta(\mathcal{M})$, and the maps in $\mathcal{E}(\Theta(\mathcal{N} \oplus \mathcal{M}))$ are of the form $P \widehat{\oplus} Q$ with $P \in \mathcal{E}(\Theta(\mathcal{N}), Q \in \mathcal{E}(\Theta(\mathcal{M})$. So $P \widehat{\oplus} Q \in \mathcal{A}(\Theta(\mathcal{N} \oplus \mathcal{M}))$ if either $P \neq i d$ or $Q \neq i d$.

We show that for $k \in \mathbb{Z}_{+}$,

$$
z_{\mathcal{N} \oplus \mathcal{M}, k}^{*}(\Phi \oplus \Psi)=\bigcup_{r=0}^{k} z_{\mathcal{N}, r}^{*}(\Phi) \oplus z_{\mathcal{M}, k-r}^{*}(\Psi) .
$$

This is obvious for $k=0$ and the inclusion $\subset$ is clear for $k=1$ and then follows for all $k \in \mathbb{N}$ by induction.

For the reverse inclusion, proceed by induction, with $k \geq 1$. Suppose that $\mathcal{N}_{1} \oplus \mathcal{M}_{1} \in \Theta(\mathcal{N} \oplus \mathcal{M})$ and $r_{1}, s_{1} \geq 0$ are the smallest integers such that $\mathcal{N}_{1} \in z_{\mathcal{N}, r_{1}}^{*}(\Phi)$ and $\mathcal{M}_{1} \in z_{\mathcal{M}, s_{1}}^{*}(\Psi)$ with $r_{1}+s_{1}>k$. We use the inductive hypothesis to show that $\mathcal{N}_{1} \oplus \mathcal{M}_{1} \notin z_{\mathcal{N} \oplus \mathcal{M}, k}^{*}(\Phi \oplus \Psi)$. Either $r_{1}$ or $s_{1}$ is at least 1. Suppose $r_{1} \geq 1$. There exists $P \in \mathcal{A}(\Theta(\mathcal{N}))$ such that $r_{2}=r_{1}-1$ is the smallest value such that $P\left(\mathcal{N}_{1}\right) \in z_{\mathcal{N}, r_{2}}^{*}(\Phi)$. Since $r_{2}+s_{1}>k-1$ it follows from the induction hypothesis that $(P \widehat{\oplus} i d)\left(\mathcal{N}_{1} \oplus \mathcal{M}_{1}\right) \notin z_{\mathcal{N} \oplus \mathcal{M}, k-1}^{*}(\Phi \oplus \Psi)$ and so $\mathcal{N}_{1} \oplus \mathcal{M}_{1} \notin z_{\mathcal{N} \oplus \mathcal{M}, k}^{*}(\Phi \oplus \Psi)$.

With $\Phi=\Psi=[[0]]$ this implies (5.21). With $\Phi=\Theta(\mathcal{N})$ we obtain for $k \in \mathbb{Z}_{+}$,

$$
z_{\mathcal{N} \oplus \mathcal{M}, k}^{*}(\Theta(\mathcal{N}) \oplus \Psi)=\Theta(\mathcal{N}) \oplus z_{\mathcal{M}, k}^{*}(\Psi)
$$

Notice that these results did not require that $\mathcal{N}$ be finite.

Now we assume that $\mathcal{N}$ is finite and for (5.22) we proceed by transfinite induction. Since $\mathcal{N}$ is positive and finite there exists $r_{0}^{*}$ such that $z_{\mathcal{N}, r_{0}^{*}}^{*}([[0]])=$ $\Theta(\mathcal{N})$. That is, the $z^{*}$ sequence stabilizes at $r_{0}^{*}$. 
Taking the union in (5.21) over $k \in \mathbb{Z}$ we obtain (5.22) with $\alpha=\omega$. Assuming the result for a limit ordinal $\alpha$ and $k=0$ it then follows for $k \in \mathbb{N}$ by (5.25) with $\Psi=z_{\mathcal{M}, \alpha}^{*}([[0]])$. Again taking the union over $k \in \mathbb{N}$ we obtain the result for $\alpha+\omega$. Finally, if the ordinal $\bar{\alpha}$ is the limit of an increasing sequence of limit ordinals $\beta^{i}$ we take the union of the (5.22) with $\alpha=\beta^{i}$ and $k=0$ to obtain the result with $\alpha=\bar{\alpha}$.

Proposition 5.33. Assume that $\left\{\mathcal{M}_{a}\right\}$ is a finite or infinite pairwise disjoint collection of at least two nonempty labels of finite type and let $\mathcal{M}=\bigcup\left\{\mathcal{M}_{a}\right\}$.

(a) For every $\alpha \geq 1$

$$
z_{L A B, \alpha}(\Theta(\mathcal{M}))=\bigcup\left\{z_{L A B, \alpha}\left(\Theta\left(\mathcal{M}_{a}\right)\right)\right\} .
$$

(b) If for all $a, \quad \Phi_{a}$ is a $\Theta$ invariant subspace of $\Theta^{\prime}\left(\mathcal{M}_{a}\right)$ then $\bigcup\left\{\Phi_{a}\right\}$ is $a \Theta$ invariant subset of $\Theta^{\prime}(\mathcal{M})$. If $\bigcup\left\{\Phi_{a}\right\}$ is a proper subset of $\Theta^{\prime}(\mathcal{M})$ then

$$
z_{\mathcal{M}}^{*}\left(\bigcup\left\{\Phi_{a}\right\}\right)=\left(\bigcup\left\{z_{\mathcal{M}_{a}}^{*}\left(\Phi_{a}\right)\right\}\right) \backslash\left\{\mathcal{M}_{a}\right\}
$$

Proof: If $\Phi$ is a closed subset of $\Theta(\mathcal{M})$ then $z_{L A B}(\Phi)$ is the closure of $\mathcal{A}(\Theta(\mathcal{M})) \Phi$. Hence, (5.26) follows by transfinite induction starting with $z_{L A B}(\Theta(\mathcal{M}))=\Theta^{\prime}(\mathcal{M})$.

The $\Theta$ invariance of $\bigcup\left\{\Phi_{a}\right\}$ and (5.27) follow from (4.12) and (4.13).

Notice that, in general, for a label $\mathcal{N}$ and $\Psi$ a $\Theta$ invariant subset of $\Theta^{\prime}(\mathcal{N})$ we have $\mathcal{N} \in z_{\mathcal{N}}^{*}(\Psi)$ if and only iff $\Psi=\Theta^{\prime}(\mathcal{N})$. In particular, $\mathcal{M}_{a} \in z_{\mathcal{M}_{a}}^{*}\left(\Theta^{\prime}\left(\mathcal{M}_{a}\right)\right)$ but $\mathcal{M}_{a} \notin \Theta(\mathcal{M})$. The proper subset condition is needed because $\mathcal{M} \in$ $z_{\mathcal{M}}^{*}\left(\Theta^{\prime}(\mathcal{M})\right)$ while $\mathcal{M} \notin \bigcup z_{\mathcal{M}_{a}}^{*}\left(\Theta^{\prime}\left(\mathcal{M}_{a}\right)\right)$

Definition 5.34. For $\mathcal{M}$ a nonzero WAP label, the height of $\Theta(\mathcal{N})$ is $\alpha+1$ where $\alpha$ is the ordinal with $z_{L A B, \alpha}(\Theta(\mathcal{M}))=[[0]]$. The height $t^{*}$ is $\alpha+1$ where $\alpha$ is the ordinal where $z_{\mathcal{M}, \alpha}^{*}(\{\emptyset\})=\Theta^{\prime}(\mathcal{M})$. Notice that $z_{\mathcal{M}, 1+\alpha}^{*}(\{\emptyset\})=z_{\mathcal{M}, \alpha}^{*}([[0]])$ and so if $\alpha \geq \omega$ then $1+\alpha=\alpha$, hence $z_{\mathcal{M}, \alpha}^{*}(\{\emptyset\})=z_{\mathcal{M}, 1+\alpha}^{*}(\{\emptyset\})=z_{\mathcal{M}, \alpha}^{*}([[0]])$.

Theorem 5.35. For any countable limit ordinal $\alpha$ there exists a label $\mathcal{M}$ which is both simple and finitary with height $=$ height $* \alpha+1$. For any countable ordinal $\alpha$ there exists a label $\mathcal{M}$ which is both simple and finitary with height $=\alpha+1$. Hence, $(X(\mathcal{M}), S)$ and $\left(X_{+}(\mathcal{M}), S\right)$ are topologically transitive WAP subshifts with height $=\alpha+1$ and, if $\alpha$ is a limit ordinal, with height ${ }^{*}=\alpha+1$. 
Proof: First, let $\mathcal{N}_{n}=\left\{k \chi\left(\ell_{1}\right): 0 \leq k \leq n\right\}$. It is easy to see that $\Theta\left(\mathcal{N}_{n}\right)=\left\{\mathcal{N}_{k}: k \leq n\right\} \cup\{\emptyset\}$ has height and height* equal to $n+1$. These are finite labels and so are both simple and finitary.

Now suppose that $\alpha$ is a countable limit ordinal, the limit of an increasing sequence $\beta^{i}$. By inductive hypothesis, we can choose for each $i$ a finitary and simple label $\mathcal{M}^{i}$ so that $\Theta\left(M_{i}\right)$ has height $\beta^{i}+1$, and height* $\beta^{i}+1$. By using a Gamow transformation we can assume that $\left\{\mathcal{M}^{i}\right\}$ is a sequence with disjoint supports. By Proposition 5.33 $\mathcal{M}=\bigcup\left\{\mathcal{M}^{i}\right\}$ is finitary and simple and by $(5.26) \zeta_{L A B, \alpha}(\Theta(\mathcal{M}))=\{[[0]]\}$ and so $\Theta(\mathcal{M})$ has height $\alpha+1$. By (5.27) it follows that $\zeta_{\mathcal{M}, \alpha}^{*}(\{\emptyset\})=\zeta_{\mathcal{M}, \alpha}^{*}([[0]])=\Theta^{\prime}(\mathcal{M})$ and so $\Theta(\mathcal{M})$ has height* equal to $\alpha+1$.

Now for a countable limit ordinal $\alpha$ assume that $\mathcal{M}$ is a finitary and simple label with height and height* equal to $\alpha+1$. By using a Gamow transformation, we can assume that $\ell_{1}$ is not in the support of $\mathcal{M}$. For $n \geq 1, \mathcal{N}_{n}$ is a positive, finite label disjoint from $\mathcal{M}$. By Proposition $5.33 \mathcal{N}_{n} \oplus \mathcal{M}$ is finitary and simple and by $(5.19) z_{L A B, \alpha}\left(\Theta\left(\mathcal{N}_{n} \oplus \mathcal{M}\right)\right)=\Theta\left(\mathcal{N}_{n} \oplus 0\right)$. Hence, $\Theta\left(\mathcal{N}_{n} \oplus \mathcal{M}\right)$ has height $\alpha+n+1$.

The results for $(X(\mathcal{M}), S)$ follow from Theorem 5.30 and Theorem 5.31.

\section{SCRAMBLED SETS}

Following Li and Yorke [36] a subset $S \subset X$ is called scrambled for a dynamical system $(X, T)$ when every pair of distinct points of $S$ is proximal but not asymptotic.

Recall that the adherence semigroup $A(X, T)$ is the ideal of the enveloping semigroup $E(X, T)$ consisting of the limit points of $\left\{T^{n}\right\}$ as $|n| \rightarrow \infty$. Let $A_{+}(X, T)$ be the set of limit points of $\left\{T^{n}\right\}$ as $n \rightarrow \infty$, that is, we move only in the positive direction. Thus, $\omega T(x)=A_{+}(X, T) x$ for every $x \in X$.

Definition 6.1. For a metric dynamical system $(X, T)$ let $(x, y)$ be a pair in $X \times X$.

(a) We call the pair $(x, y)$ proximal when it satisfies the following equivalent conditions:

(i) $\liminf _{n>0} d\left(T^{n}(x), T^{n}(y)\right)=0$.

(ii) There exists a sequence $n_{i} \rightarrow \infty$ such that $\lim d\left(T^{n_{i}}(x), T^{n_{i}}(y)\right)=0$.

(iii) There exists $p \in A_{+}(X, T)$ such that $p x=p y$.

(iv) There exists $u$ a minimal idempotent in $A_{+}(X, T)$ such that $u x=u y$.

We denote by $\operatorname{PROX}(X, T)$ (or just $P R O X$ when the system is clear) the set of all proximal pairs. 
(b) We call the pair $(x, y)$ asymptotic when it satisfies the following equivalent conditions:

(i) $\lim \sup _{n>0} d\left(T^{n}(x), T^{n}(y)\right)=0$.

(ii) $\lim _{n>0} d\left(T^{n}(x), T^{n}(y)\right)=0$.

(iii) For all $p \in A_{+}(X, T) p x=p y$.

We denote by $A S Y M P(X, T)$ (or just $A S Y M P$ when the system is clear) the set of all asymptotic pairs.

(c) We call the pair $(x, y)$ a Li-Yorke pair when it is proximal but not asymptotic.

(d) The system $(X, T)$ is called proximal when all pairs are proximal, i.e. $P R O X=X \times X$. It is called completely scrambled when all non diagonal pairs are Li-Yorke. That is, the system is proximal, but $A S Y M P=\Delta_{X}$.

Observe that the set $\left\{p \in A_{+}(X, T): p x=p y\right\}$ is a closed left ideal if it is nonempty and so it then contains minimal idempotents. This shows that $(i i i) \Leftrightarrow(i v)$ in $(\mathrm{a})$. The remaining equivalences are obvious.

Remark 6.2. This notion of proximality actually refers to the action of the semigroup $\left\{T^{n}: n \in \mathbb{Z}_{+}\right\}$. The usual definition of proximality would be: $x$ and $y$ are proximal if there exists a sequence $n_{i} \in \mathbb{Z}$ with $\left|n_{i}\right| \rightarrow \infty$ such that $\lim d\left(T^{n_{i}}(x), T^{n_{i}}(y)\right)=0$.

Lemma 6.3. For $x \in X$ and $n \in \mathbb{N}$ the pair $\left(x, T^{n}(x)\right)$ is proximal iff $T^{n}(y)=$ $y$ for some $y \in \omega T(x)$. The pair $\left(x, T^{n}(x)\right)$ is asymptotic iff $T^{n}(y)=y$ for every $y \in \omega T(x)$.

Proof: $p T^{n}(x)=T^{n}(p x)$ and so $p x=p T^{n}(x)$ iff $p x=T^{n}(p x)$. The pair is proximal (or asymptotic) iff $p x=T^{n}(p x)$ for some $p \in A_{+}(X, T)$ (resp. for all $\left.p \in A_{+}(X, T)\right)$.

We recall the following, see, e.g. [8, Proposition 2.2].

Proposition 6.4. A metric dynamical system $(X, T)$ is proximal iff there exists a fixed point $e \in X$ which is the unique minimal subset of $X$, i.e. $(X, T)$ is a minCT system. Consequently, $\left(X, T^{-1}\right)$ is proximal if $(X, T)$ is.

Proof: If $u$ is a minimal idempotent then $u x$ is a minimal point for every $x \in X$. So if $e$ is the unique minimal point of $X$, then $u x=e$ for every $x \in X$ and every minimal idempotent $u$. Hence, every pair is proximal.

Assume now that $(X, T)$ is proximal. For any $x \in X$, the pair $(x, T(x))$ is proximal and so there exists $p \in A_{+}(X, T)$ such that $p x=p T(x)=T(p x)$ and so $e=p x$ is a fixed point. A pair of distinct fixed points is not proximal and 
so $e$ is the unique fixed point. Hence, $e$ is in the orbit closure of every point and so $\{e\}$ is the only minimal set.

Since the minimal subsets for $T$ and $T^{-1}$ are the same, it follows that $\left(X, T^{-1}\right)$ is proximal.

Thus, we obtain the following obvious corollary. Compare Proposition 1.20

Corollary 6.5. A metric dynamical system $(X, T)$ is completely scrambled iff it is a minCT system and $A_{+}(X, T)$ distinguishes points of $X$.

Completely scrambled systems were introduced by Huang and Ye [31] who provided a rich supply of examples, but all appear to be of height the first countable ordinal.

In contrast with proximal systems there exist completely scrambled systems $(X, T)$ whose inverse $\left(X, T^{-1}\right)$ is not completely scrambled.

Example 6.6. Begin with $(Y, F)$ a completely scrambled system with fixed point $e$. Let $(X, T)$ be the quotient space of the product system $(Y \times\{0,1\}, F \times$ $\left.i d_{\{0,1\}}\right)$ obtained by identifying $(e, 0)$ with $(e, 1)$ to obtain the fixed point denoted $e$ in $X$. Let $X_{0}$ and $X_{1}$ be the images of $Y \times\{0\}$ and $Y \times\{1\}$ in $X$. Since $(X, T)$ has a unique fixed point $e$ we can construct a sequence $\left\{x^{n}: n \in \mathbb{Z}_{+}\right\}$ so that

- $x^{0}=e$.

- $\left\{d\left(x^{n}, T\left(x^{n}\right)\right)\right\} \rightarrow 0$ as $n \rightarrow \infty$.

- For every $N \in \mathbb{N}$ the set $\left\{x^{i}: i \geq N\right\}$ is dense in $X$.

Now for $n \in \mathbb{Z}$ let $z^{n} \in X \times[0,1]$ be defined by

$$
z^{n}=\left\{\begin{array}{lc}
\left(x^{n}, 1 /(n+1)\right) & \text { for } n \geq 0 \\
(e, 1 /(-n+1)) & \text { for } n<0
\end{array}\right.
$$

Let

$$
\hat{X}=X \times\{0\} \cup\left\{z^{n}: n \in \mathbb{Z}\right\} .
$$

Let $\hat{T}(x, 0)=(T(x), 0)$ and $\hat{T}\left(z^{n}\right)=z^{n+1}$.

The system $(\hat{X}, \hat{T})$ is topologically transitive with fixed point $(e, 0)$ the unique minimal set. Hence, the system is proximal. Since every orbit in $X$ is confined to either $X_{0}$ or $X_{1}$ it follows that no point $z^{n}$ is asymptotic to a point in $X \times\{0\}$. By Lemma 6.3 no two distinct points on the $z^{n}$ orbit are asymptotic. Hence, $(\hat{X}, \hat{T})$ is completely scrambled. However, the inverse $\left(\hat{X}, \hat{T}^{-1}\right)$ is not since $\left\{z^{n}\right\} \rightarrow(e, 0)$ as $n \rightarrow-\infty$. 
By a result of Schwartzman (see Gottschalk and Hedlund [30, Theorem 10.36]) a nontrivial, expansive system admits non-diagonal asymptotic pairs. It follows that no nontrivial subshift can be completely scrambled. However, we note that the subshifts which arise from labels of finite type are pretty close.

Proposition 6.7. If $\mathcal{M}_{1}, \mathcal{M}_{2}$ are two different labels then the following are equivalent.

(i) For some $n_{1}, n_{2} \in \mathbb{Z}$ the pair $\left(S^{n_{1}} x\left[\mathcal{M}_{1}\right], S^{n_{2}} x\left[\mathcal{M}_{2}\right]\right)$ is asymptotic for $S$ or $S^{-1}$.

$(\mathrm{i}+)$ For some $n_{1}, n_{2} \in \mathbb{Z}$ the pair $\left(S^{n_{1}} x_{+}\left[\mathcal{M}_{1}\right], S^{n_{2}} x_{+}\left[\mathcal{M}_{2}\right]\right)$ is asymptotic for $S$ or $S^{-1}$.

(ii) For all $n_{1}, n_{2} \in \mathbb{Z}$ the pairs $\left(S^{n_{1}} x\left[\mathcal{M}_{1}\right], S^{n_{2}} x\left[\mathcal{M}_{2}\right]\right)$ are asymptotic for both $S$ and $S^{-1}$.

(ii+) For all $n_{1}, n_{2} \in \mathbb{Z}$ the pairs $\left(S^{n_{1}} x_{+}\left[\mathcal{M}_{1}\right], S^{n_{2}} x_{+}\left[\mathcal{M}_{2}\right]\right)$ are asymptotic for both $S$ and $S^{-1}$.

(iii) $\left\{\mathcal{M}_{1}, \mathcal{M}_{2}\right\}=\{\emptyset, 0\}$.

Proof: Since $R_{S}(x[0])=e=x[\emptyset]$, it is clear that (iii) implies (ii), (ii+). That (ii) implies (i) and (ii+) implies (i+) are obvious.

Now assume that $\left\{\mathcal{M}_{1}, \mathcal{M}_{2}\right\} \neq\{\emptyset, 0\}$. By renumbering we can assume that there exists $\mathbf{r}>\mathbf{0}$ such that $\mathbf{r} \in \mathcal{M}_{1} \backslash \mathcal{M}_{2}$. Let $\left\{t^{i} \in I P_{+}(k)\right\}$ be a sequence with length vector $\mathbf{r}$ and such that $\left\{j_{r}\left(t^{i}\right) \rightarrow \infty\right.$. Then by Theorem 3.25 $\operatorname{Lim} S^{t^{i}}\left(x\left[\mathcal{M}_{1}\right]\right)=x\left[\mathcal{M}_{1}-\mathbf{r}\right]$ and $\operatorname{Lim} S^{t^{i}}\left(x_{+}\left[\mathcal{M}_{1}\right]\right)=x_{+}\left[\mathcal{M}_{1}-\mathbf{r}\right]$. Neither limit point is the fixed point $e=x[\emptyset]$ since $\mathbf{r} \in \mathcal{M}_{1}$. Hence, for any $n_{1} \in \mathbb{Z}$, $\operatorname{Lim} S^{t^{i}}\left(S^{n_{1}}\left(x\left[\mathcal{M}_{1}\right]\right)\right)=S^{n_{1}}\left(x\left[\mathcal{M}_{1}-\mathbf{r}\right]\right) \neq e$. On the other hand, since, $\mathbf{r} \notin \mathcal{M}_{2}$

$$
\operatorname{Lim} S^{t^{i}}\left(S^{n_{2}}\left(x\left[\mathcal{M}_{2}\right]\right)\right)=S^{n_{2}}\left(x\left[\mathcal{M}_{2}-\mathbf{r}\right]\right)=e
$$

and

$$
\operatorname{Lim} S^{t^{i}}\left(S^{n_{2}}\left(x_{+}\left[\mathcal{M}_{2}\right]\right)\right)=S^{n_{2}}\left(x_{+}\left[\mathcal{M}_{2}-\mathbf{r}\right]\right)=e .
$$

Thus, the pairs $\left(S^{n_{1}} x\left[\mathcal{M}_{1}\right], S^{n_{2}} x\left[\mathcal{M}_{2}\right]\right)\left(S^{n_{1}} x_{+}\left[\mathcal{M}_{1}\right], S^{n_{2}} x_{+}\left[\mathcal{M}_{2}\right]\right)$ are not asymptotic for $S$ or for $S^{-1}$. This prove the contrapositive of $(i),(i+) \Rightarrow(i i i)$.

Corollary 6.8. For any positive label $\mathcal{M}$ the set $\left\{S^{n}(x[\mathcal{N}]): 0 \neq \mathcal{N} \in\right.$ $\Theta(\mathcal{M}), n \in \mathbb{Z}\}$ is a scrambled subset for $(X(\mathcal{M}), S)$ and for $\left(X(\mathcal{M}), S^{-1}\right)$. The set $\left\{S^{n}\left(x_{+}[\mathcal{N}]\right): 0 \neq \mathcal{N} \in \Theta(\mathcal{N}), n \in \mathbb{Z}\right\}$ is a scrambled subset for $\left(X_{+}(\mathcal{M}), S\right)$ and for $\left(X_{+}(\mathcal{M}), S^{-1}\right)$. If $\mathcal{M}$ is a label of finite type then these sets are the complement of the orbit of $x[0]$ in $X(\mathcal{M})$ and $X_{+}(\mathcal{M})$, respectively. 
Proof: That the set is scrambled is clear from Proposition 6.7. In the finite type case, Corollary 3.43 (a) implies that we are excluding only the orbit of $x[0]$ from the set.

Definition 6.9. An inverse sequence in $\mathcal{L A B}$ is a sequence $\left\{\mathcal{M}^{i}, \mathbf{r}^{i}: i \in \mathbb{Z}_{+}\right\}$ with $\mathbf{r}^{i}>\mathbf{0}$ in $\mathcal{M}^{i}$ and such that $\mathcal{M}^{i}=\mathcal{M}^{i+1}-\mathbf{r}^{i+1}$ for $i>0$. For the associated inverse sequences $p_{\mathbf{r}^{i+1}}:\left(X\left(\mathcal{N}^{i+1}\right), S\right) \rightarrow\left(X\left(\mathcal{M}^{i}\right), S\right)$ and $p_{\mathbf{r}^{i+1}}$ : $\left(X_{+}\left(\mathcal{M}^{i+1}\right), S\right) \rightarrow\left(X_{+}\left(\mathcal{M}^{i}\right), S\right)$ we let $\left(X\left(\left\{\mathcal{M}^{i}, \mathbf{r}^{i}\right\}\right), S\right)$ and $\left(X_{+}\left(\left\{\mathcal{M}^{i}, \mathbf{r}^{i}\right\}\right), S\right)$ denote the respective inverse limits.

Theorem 6.10. Let $\left\{\mathcal{M}^{i}, \mathbf{r}^{i}\right\}$ be an inverse sequence in $\mathcal{L} \mathcal{A B}$. The inverse limit system $\left(X\left(\left\{\mathcal{M}^{i}, \mathbf{r}^{i}\right\}\right), S\right)$ and $\left(X_{+}\left(\left\{\mathcal{N}^{i}, \mathbf{r}^{i}\right\}\right), S\right)$ are topologically transitive, compact metrizable systems. If each $\mathcal{M}^{i}$ is of finite type then the limit systems and their inverses are completely scrambled. If each $\mathcal{M}^{i}$ is either finitary or simple then the limit systems are WAP.

Proof: Topologically transitive and WAP systems are closed under inverse limits. For the latter, notice that the inverse limit is a subsystem of the product which is clearly WAP. In this case, each map $p_{\mathbf{r}^{i+1}}$ is surjective as required because it maps the transitive point $x\left[\mathcal{M}^{i+1}\right]$ of $X\left(\mathcal{M}^{i+1}\right)$ onto the transitive point $x\left[\mathcal{M}^{i}\right]$ of $X\left(\mathcal{M}^{i}\right)$ since $\mathcal{M}^{i}=\mathcal{M}^{i+1}-\mathbf{r}^{i+1}$. In particular, the sequence $x^{*}=\left\{x\left[\mathcal{M}^{i}\right]\right\}$ is a transitive point for the inverse limit.

The point $e$ associated with the sequence $\{x[\emptyset]\}$ is a fixed point in $X\left(\left\{\mathcal{N}^{i}, \mathbf{r}^{i}\right\}\right)$. A minimal subset of the limit space projects to a minimal subset of $X\left(\mathcal{M}^{i}\right)$ for each $i$. If $\mathcal{M}^{i}$ is of finite type then this minimal subset is $\{e\} \subset X\left(\mathcal{M}^{i}\right)$. Thus, if all are of finite type, the fixed point is the only minimal subset of the limit and so $\left(X\left(\left\{\mathcal{N}^{i}, \mathbf{r}^{i}\right\}\right), S\right)$ is proximal by Proposition 6.4 .

Notice that if $x \in X\left(\mathcal{M}^{i}\right)$ is not equal to the fixed point $e$ then $x[0] \notin$ $p_{\mathbf{r}^{i+1}}^{-1}(x)$. If $x, y$ are distinct points of $X\left(\left\{\mathcal{M}^{i}, \mathbf{r}^{i}\right\}\right)$ then for sufficiently large $i$ they project to distinct points of $X\left(\mathcal{N}^{i}\right)$ with neither projecting to $x[0]$ in $X\left(\mathcal{M}^{i}\right)$. In the finite type case it then follows from Corollary 6.8 that for sufficiently large $i, x$ and $y$ project to a non-asymptotic pair. Consequently, the pair $(x, y)$ is not asymptotic in $X\left(\left\{\mathcal{M}^{i}, \mathbf{r}^{i}\right\}\right)$.

Remark 6.11. Since a transitive point for $X\left(\left\{\mathcal{N}^{i}, \mathbf{r}^{i}\right\}\right)$ projects to a transitive point on each $X\left(\mathcal{M}^{i}\right)$ it follows that the transitive points for $X\left(\left\{\mathcal{M}^{i}, \mathbf{r}^{i}\right\}\right)$ are all on the orbit of $x^{*}$ described above and so $x^{*}$ is isolated when the labels $\mathcal{M}^{i}$ are of finite type. Similarly, for $X_{+}\left(\left\{\mathcal{M}^{i}, \mathbf{r}^{i}\right\}\right)$. 
For the construction of our examples, we need the following. Recall that 5.32 implies that if $\mathcal{M}_{1}$ is a finite label and $\mathcal{M}_{2}$ is a label with supports disjoint from those of $\mathcal{M}_{1}$, then

$$
\Theta^{\prime}\left(\mathcal{M}_{1} \oplus \mathcal{M}_{2}\right)=\Theta^{\prime}\left(\mathcal{M}_{1}\right) \oplus \Theta\left(\mathcal{M}_{2}\right) \cup \Theta\left(\mathcal{M}_{1}\right) \oplus \Theta^{\prime}\left(\mathcal{M}_{2}\right)
$$

Lemma 6.12. Let $\mathbf{r}$ be a positive finite vector with support disjoint from those in Supp $\mathcal{M}$ for some nonempty label $\mathcal{M}$. Then $P_{\mathbf{r}}\left(\Theta^{\prime}(\langle\mathbf{r}\rangle) \oplus \Theta(\mathcal{M})\right)=\{\emptyset\}$ and on $\{\langle\mathbf{r}\rangle\} \oplus \Theta(\mathcal{M}) \quad P_{\mathbf{r}}$ is a bijection onto $\Theta(\mathcal{M})$.

Proof: Since $\mathbf{r}$ is not an element of any label in $\Theta^{\prime}(\langle\mathbf{r}\rangle) \oplus \Theta(\mathcal{M})$ it follows that all of these labels are mapped to $\emptyset$.

By 5.32 every label of $\Theta(\langle\mathbf{r}\rangle \oplus \mathcal{M})$ is of the form $\mathcal{N}_{1} \oplus \mathcal{N}_{2}$ with $\mathcal{N}_{1} \in \Theta(\langle\mathbf{r}\rangle)$ and $\mathcal{N}_{2} \in \Theta(\mathcal{M})$. If $\mathcal{N}_{1} \neq\langle\mathbf{r}\rangle$ then $P_{\mathbf{r}}$ maps $\mathcal{N}_{1} \oplus \mathcal{N}_{2}$ to $\emptyset$. If $\mathcal{N}_{1}=\langle\mathbf{r}\rangle$ then $P_{\mathbf{r}}$ maps $\mathcal{N}_{1} \oplus \mathcal{N}_{2}$ to $\mathcal{N}_{2}$. Hence, for any $\mathcal{N}_{2} \in \Theta(\mathcal{M})$ the unique label of the form $\langle\mathbf{r}\rangle \oplus \mathcal{N}$ which is mapped to $\mathcal{N}_{2}$ has $\mathcal{N}=\mathcal{N}_{2}$.

Example 6.13. Let $\left\{\mathbf{r}^{i}\right\}$ be a sequence of positive $\mathbb{N}$-vectors all with disjoint supports and let $\mathcal{M}$ be a finitary label with the sets in Supp $\mathcal{M}$ disjoint from the supports of the sequence.

Let $\mathcal{N}^{0}=\{0\}$ and $\mathcal{N}^{i+1}=\left\langle\mathbf{r}^{i+1}\right\rangle \oplus \mathcal{N}^{i}$ define an increasing sequence of finite labels. Define $\left\{\mathcal{N}^{i}=\mathcal{N}^{i} \oplus \mathcal{M}, \mathbf{r}^{i}\right\}$, an inverse sequence of finitary labels. For each $i$ Lemma 6.12 implies that the preimage of $\emptyset$ by $P_{\mathbf{r}^{i+1}}: \Theta\left(\mathcal{M}^{i+1}\right) \rightarrow \Theta\left(\mathcal{M}^{i}\right)$ is countable and the preimage of every other point is a singleton. It follows that the limit system $\left(X\left(\left\{\mathcal{M}^{i}, \mathbf{r}^{i}\right\}\right), S\right)$ and its inverse $\left(X\left(\left\{\mathcal{M}^{i}, \mathbf{r}^{i}\right\}\right), S^{-1}\right)$ are completely scrambled, topologically transitive, countable WAPs.

Notice that $X(\mathcal{M})$ and $X_{+}(\mathcal{M})$ are factors of $X\left(\left\{\mathcal{M}^{i}, \mathbf{r}^{i}\right\}\right)$ and $X_{+}\left(\left\{\mathcal{M}^{i}, \mathbf{r}^{i}\right\}\right)$ respectively. Hence, if we choose $\mathcal{M}$ with height greater than some countable ordinal $\alpha$ then $X\left(\left\{\mathcal{M}^{i}, \mathbf{r}^{i}\right\}\right)$ and $X_{+}\left(\left\{\mathcal{M}^{i}, \mathbf{r}^{i}\right\}\right)$ have height greater than $\alpha$.

Corollary 6.14. For every countable ordinal $\alpha$ there exist a topologically transitive completely scrambled, countable WAP system of height greater than $\alpha$.

Following Huang and Ye we can take countable products of copies of these examples to get completely scrambled WAP systems on the Cantor set with arbitrarily large heights. However, these examples will not be topologically transitive. 


\section{Appendix A. Directed Sets And nets}

We review the theory of nets, following [34, Chapter 2].

A directed set is a set $I$ equipped with a reflexive, transitive relation $\prec$ such that if $i_{1}, i_{2} \in I$ then there exists $j \in I$ such that $i_{1}, i_{2} \prec j$.

For $i \in I$ let $\prec_{i}=\{j: i \prec j\}$. A set $F \subset I$ is called terminal if $F \supset \prec_{i}$ for some $i \in I$. F is called cofinal if $F \cap \prec_{i} \neq \emptyset$ for all $i \in I$. In the family language of [2] these are dual families of subsets of $I$. Because the set $I$ is directed by $\prec$ it follows that the family of terminal sets is a filter. That is, a finite intersection of terminal sets is terminal. The cofinal sets satisfy the dual, Ramsey Property: If a finite union of subsets of $I$ is cofinal then at least one of them is cofinal.

For example, if $x$ is a point of a space $X$ then the set $\mathcal{N}_{x}$ of neighborhoods of $x$ is directed by $\supset$ and a subset of $\mathcal{N}_{x}$ is cofinal iff it is a neighborhood base. The sets $\mathbb{Z}_{+}$and $\mathbb{N}$ are directed by $\leq$ and a subset is terminal iff it is cofinite. A subset is cofinal iff it is infinite.

A net in a set $Q$ is a function from a directed set $I$ to $Q$, denoted $\left\{x^{i}: i \in I\right\}$. If $A \subset Q$ we say that the net is eventually (or frequently) in $A$ if $\left\{i: x^{i} \in A\right\}$ is terminal (resp. is cofinal). If $x$ is a point of a space $X$ then a net in $X$ converges to $x$ (or has $x$ is a limit point) if for every $U \in \mathcal{N}_{x}$ the net is eventually in $U$ (resp. is frequently in $U$ ). Thus, if a net in $A$ has $x$ as a limit point then $x$ is in the closure of $A$. Conversely, if $x \in \bar{A}$ then we can use $I=\mathcal{N}_{x}$ and choose $x^{U} \in A \cap U$. We thus obtain a net in $A$ converging to $x$.

If $x$ does not have a countable neighborhood base there may be no sequence in $A$ which converges to $x$. For example, if $X=\beta Q$ for some infinite set $Q$ then no infinite subset $Q_{0}$ of $Q$ has a unique limit point because, by Lemma C.1 the inclusion of $Q_{0}$ into $Q$ extends to a homeomorphism of $\beta Q_{0}$ onto the closure of $Q_{0}$.

A map $k: I^{\prime} \rightarrow I$ between directed sets is a directed set morphism if $k^{-1}(F)$ is terminal in $I^{\prime}$ whenever $F$ is terminal in $I$. If $i_{1}^{\prime} \prec i_{2}^{\prime}$ implies $k\left(i_{1}^{\prime}\right) \prec k\left(i_{2}^{\prime}\right)$ and the image, $k\left(I^{\prime}\right)$, is cofinal in $I$ then $k$ is a morphism.

A map $k: I^{\prime} \rightarrow I$ is a morphism iff whenever $F$ is cofinal in $I^{\prime}$, then $k(F)$ is cofinal in $I$. This follows because

$$
k(F) \cap A \neq \emptyset \quad \Longleftrightarrow F \cap k^{-1}(A) \neq \emptyset
$$

and a set is cofinal iff it meets every terminal set and vice-versa.

With this definition of morphism, the class of directed sets becomes a category.

If $i \mapsto x^{i}$ is a net, then the composite $i^{\prime} \mapsto x^{k\left(i^{\prime}\right)}$ is the subnet induced by $k$. We will usually suppress the mention of $k$ and just write $\left\{x^{i^{\prime}}: i^{\prime} \in I^{\prime}\right\}$ for the subnet. Their use is illustrated by the following result. 
Lemma A.1. (The Smith Lemma) Let $\mathcal{A}$ be a collection of nonempty subsets of of a set $Q$ which is closed under finite intersection and let $\left\{x^{i}: i \in I\right\}$ be a net in $Q$. If $x^{i} \in A$ frequently for every $A \in \mathcal{A}$, then there is a subnet $\left\{x^{i^{\prime}}: i^{\prime} \in I^{\prime}\right\}$ such that $x^{i^{\prime}} \in A$ eventually for every $A \in \mathcal{A}$. Conversely, if such a subnet exists then $x^{i} \in A$ frequently for every $A \in \mathcal{A}$.

Proof: By assumption, $\mathcal{A}$ is directed by $\supset$. On the product $\mathcal{A} \times I$ we use the product ordering $\left(A_{1}, i_{i}\right) \supset \times \prec\left(A_{2}, i_{2}\right)$ if $A_{1} \supset A_{2}$ and $i_{1} \prec i_{2}$. Let

$$
I^{\prime}=\left\{(A, i) \in \mathcal{A} \times I: x^{i} \in A\right\} .
$$

From the assumptions it is easy to check that

- $I^{\prime}$ is directed by $\supset \times \prec$.

- The coordinate projection $(A, i) \mapsto i$ is a morphism from $I^{\prime}$ to $I$.

- For the induced subnet $\left\{x^{i^{\prime}}: i^{\prime} \in I^{\prime}\right\} \quad x^{i^{\prime}} \in A$ eventually for every $A \in \mathcal{A}$.

For details, see [34, Lemma 2.5].

The converse follows because a terminal subset of $I^{\prime}$ maps to a cofinal subset of $I$.

Corollary A.2. If $\left\{t^{i}\right\}$ is a net in $\mathbb{Z}_{+}$then exactly one of the following is true.

(i) There is a subnet $\left\{t^{i^{\prime}}\right\}$ with limit $\infty$.

(ii) There is a finite $F \subset \mathbb{Z}_{+}$such that eventually $t^{i} \in F$ and for each $s \in F, t^{i}=s$ frequently.

Proof: Let $\mathcal{A}$ be the collection of cofinite subsets of $\mathbb{Z}_{+}$. By Lemma A.1, case (i) occurs when eventually $t^{i} \in A$ for every $A \in \mathcal{A}$. Otherwise, there exists $A \in \mathcal{A}$ such that $\left\{i: t^{i} \in A\right\}$ is not cofinite and so $\left\{i: t^{i} \in F_{0}\right\}$ is terminal for $F_{0}$ the complement of $A$. Let $F_{1}=\left\{s \in F_{1}:\left\{i: t^{i}=s\right\}\right.$ is not cofinal $\}$. So for each $s$ in the finite set $F_{1}$, eventually $t^{i} \neq s$. Let $F=F_{0} \backslash F_{1}$. By definition $\left\{i: t^{i}=s\right\}$ is cofinal for each $s \in F$. Finally, $\left\{i: t^{i} \in F\right\}$ is the intersection of a finite collection of terminal sets and so is terminal.

\section{Appendix B. Ellis semigroups And Ellis aCtions}

We will follow the notation of [2]. 
We write for a map $\phi: S \times X \rightarrow X$

$$
\begin{aligned}
& p x= \phi(p, x)=\phi^{p}(x)=\phi_{x}(p) \quad \text { for }(p, x) \in S \times X . \\
& A B=\{p x: p \in A \text { and } x \in B\} \quad \text { for } A \times B \subset S \times X . \\
& \phi^{\#}: S \rightarrow X^{X} \quad \text { is defined by } p \mapsto \phi^{p} . \\
& \phi_{\#}: X \rightarrow X^{S} \quad \text { is defined by } x \mapsto \phi_{x} .
\end{aligned}
$$

A semigroup $S$ is a nonempty set equipped with $M: S \times S \rightarrow S$ which is an associative multiplication, i.e.

$$
M^{p} \circ M^{q}=M^{p q} \quad \text { for all } p, q \in S .
$$

An action of a semigroup $S$ on a nonempty set $X$ is a map $\phi: S \times X \rightarrow X$ which is an action, i.e.

$$
\phi^{p} \circ \phi^{q}=\phi^{p q} \quad \text { for all } p, q \in S .
$$

If $S$ is a semigroup then the multiplication map $M$ is an action of $S$ on itself, called the translation action. If $X$ is a singleton set then the unique map from $S \times X$ to $X$ is an action called the trivial action.

A semigroup is called a monoid when it contains a (necessarily unique) twosided identity element, $u$, i.e. $u p=p=p u$ for all $p \in S$. A monoid action of a monoid $S$ on a set $X$ is a semi-group action such that the identity element acts as the identity map on $X$, i.e. $\phi^{u}=i d_{X}$. The translation action of a monoid is a monoid action as is the trivial action of any monoid.

If $S, T$ are semigroups then $g: S \rightarrow T$ is a semigroup homomorphism when $g(p q)=g(p) g(q)$ for all $p, q \in S$. A nonempty subset $H \subset S$ is a subsemigroup when it is closed under multiplication, i.e. $H H \subset H$, in which case the inclusion of $H$ into $S$ is a homomorphism. $H$ is an ideal when it satisfies the stronger condition $S H \subset H$. The image $g(H) \subset T$ is a subsemigroup and the preimage of a subsemigroup of $T$ is a subsemigroup of $S$ when it is nonempty. A singleton $\{u\}$ is a subsemigroup iff $u$ is an idempotent, i.e. $u u=u$. If $S, T$ are monoids then $g: S \rightarrow T$ is a monoid homomorphism when it is a semigroup homomorphism which maps the identity of $S$ to that of $T$. If $S$ is a monoid with identity $u$ and $g: S \rightarrow T$ is a surjective semigroup homomorphism then $g(u)$ is an identity in $T$. That is, $T$ is a monoid and $g$ is a monoid homomorphism. A subsemigroup of a monoid $S$ is a submonoid when it contains the identity element of $S$.

If $\phi: S \times X \rightarrow X$ and $\psi: S \times Y \rightarrow Y$ are semigroup actions, then $\pi: X \rightarrow Y$ is an action map when $\pi(p x)=p \pi(x)$ for all $p \in S, x \in X$. A subset $K \subset X$ is called invariant if $K \neq \emptyset$ and $S K \subset K$. In that case, the restricted map $\phi \mid K: S \times K \rightarrow K$ is a semigroup action and the inclusion of $K$ into $X$ is an action map. We call $\phi \mid K$ or just $K$ a subsystem of $X$. The image $\pi(K) \subset Y$ is then invariant and the preimage of an invariant subset of $Y$ is an invariant 
subset of $X$ if it is nonempty. The orbit of a point $x \in X$ is the invariant set $S x=\phi_{x}(S)$. Any union of invariant sets is invariant and any nonempty intersection of invariant sets is invariant. A subset $H \subset S$ is invariant under the translation action exactly when it is an ideal.

For any nonempty set $X$ map composition gives $X^{X}$ the structure of a monoid and the evaluation map $E v: X^{X} \times X \rightarrow X$ is a monoid action. If $\phi: S \times X \rightarrow X$ is a semigroup action then $\phi^{\#}: S \rightarrow X^{X}$ is a homomorphism and for each $x \in X, \phi_{x}=E v_{x} \circ \phi^{\#}: S \rightarrow X$ is an action map (with the translation action on $S$ ) whose image is the orbit of $x$.

If a semigroup $S$ acts on each member of an indexed family $\left\{X_{i}: i \in I\right\}$ then the product action on the product $\Pi\left\{X_{i}: i \in I\right\}$ is uniquely defined by the condition that for every $j \in I$ the projection map $\pi_{j}: \Pi\left\{X_{i}: i \in I\right\} \rightarrow X_{j}$ is an action map. In particular, if $\phi: S \times X \rightarrow X$ is an action and $I$ is any set then we obtain the product action $\phi^{I}: S \times X^{I} \rightarrow X^{I}$. We denote by $\phi^{2}$ the product action on $X \times X$, i.e. the special case with $I=\{0,1\}$. When $I=X$ we have $\left(\phi^{X}\right)_{i d_{X}}=\phi^{\#}$.

Recall that our spaces are all assumed to be nonempty and Hausdorff. For a compact space $X$ and a set $S, X^{S}$ denotes the set of all maps from $S$ to $X$, equipped with the compact, product topology.

An Ellis semigroup is a compact space equipped with a semigroup multiplication $M$ such that the adjoint map $M^{\#}: S \rightarrow S^{S}$ is continuous, or, equivalently, $M_{q}: S \rightarrow S$ is continuous for each $q \in S$. For any compact space $X$ the space of maps $X^{X}$ is an Ellis semigroup.

An Ellis action is a semigroup action $\phi$ of an Ellis semigroup on a compact space $X$ such that the semigroup homomorphism $\phi^{\#}: S \rightarrow X^{X}$ is continuous, i.e. for each $x \in X$, the action map $\phi_{x}: S \rightarrow X$ is continuous. The translation action and trivial actions of an Ellis semigroup are Ellis actions as is the evaluation action of $X^{X}$ on a compact space $X$.

Thus, $\phi$ is an Ellis action exactly when $\phi^{\#}: S \rightarrow X^{X}$ is continuous homomorphism of Ellis semigroups. The image of $\phi^{\#}$ is called the enveloping semigroup of $\phi$ and is denoted $E(\phi)$.

The idempotents play a special role in the theory because of the following crucial property of an Ellis semigroup (see [13, Lemma 2.9] and [9, Lemma $6.6])$.

Lemma B.1. Ellis-Numakura A closed subsemigroup A of an Ellis semigroup $S$ contains idempotents.

Proof: We recall the quick proof. By Zorn's Lemma we may assume that $A$ is a minimal nonempty, closed subsemigroup. Let $p \in A$. $A p \subset A$ is a closed subsemigroup and so by minimality $A p=A$. Hence, the closed subsemigroup $\{q \in A: q p=p\}$ is nonempty and so equals $A$. Hence, $p p=p$ (and $A=\{p\}$ by minimality). 


\section{Appendix C. The Stone-Čech compactification}

If $Q$ is an infinite set, which we regard as a space with the discrete topology, then the Stone-Cech Compactification is a compact space $\beta Q$ and a bijection $j$ from $Q$ onto a discrete, dense subset of $\beta Q$ which satisfies the following:

- Extension Condition If $h: Q \rightarrow X$ is a map with $X$ a compact space, then there exists a -necessarily unique- continuous map $\beta h: \beta Q \rightarrow X$ such that $h=\beta h \circ j$.

We will usually regard $j$ as an identification and so regard $Q$ as a discrete, dense subset of the compact space $\beta Q$.

The construction is a functor from the category of sets to that of compact spaces. If $q: Q_{1} \rightarrow Q_{2}$ is an map then composing with the inclusion $j_{2}$ and extending, we obtain $\beta q: \beta Q_{1} \rightarrow \beta Q_{2}$ such that $\beta q \circ j_{1}=j_{2} \circ q$. The functor properties: $\beta i d_{Q}=i d_{\beta Q}$ and $\beta(q \circ \tilde{q})=\beta q \circ \beta \tilde{q}$ follow from the uniqueness of the extension of a continuous map from a dense subset when the spaces are Hausdorff.

Lemma C.1. If $q: Q_{1} \rightarrow Q_{2}$ is injective, then $\beta q: \beta Q_{1} \rightarrow \beta Q_{2}$ restricts to a homeomorphism onto its image, the closure $\overline{q\left(Q_{1}\right)} \subset \beta Q_{2}$.

Proof: First extend the map $q^{-1}: q\left(Q_{1}\right) \rightarrow \beta Q_{1}$ arbitrarily to a map on $Q_{2}$. Then apply the Extension Condition to obtain a continuous map from $\beta Q_{2} \rightarrow \beta Q_{1}$. If $r$ is the restriction of this continuous map to $\overline{q\left(Q_{1}\right)}$, then $r \circ q$ is the identity on $Q_{1}$ and $\beta q \circ r$ is the identity on $q\left(Q_{1}\right)$. Hence, $r: \overline{q\left(Q_{1}\right)} \subset \beta Q_{2}$ is the inverse of $\beta q$.

Corollary C.2. A sequence $\left\{q^{n}: n \in \mathbb{N}\right\}$ in $Q$ is convergent in $\beta Q$ iff it is eventually constant and so converges to an element of $Q$.

Proof: If a sequence in a Hausdorff space takes on only finitely many values then it is convergent iff it is eventually constant. If $Q_{0}$ is a countably infinite subset of $Q$ then the inclusion of $Q_{0}$ into $Q$ extends to a homeomorphism of $\beta Q_{0}$ into $\beta Q$. Since $\beta Q_{0}$ is uncountable, it follows that a sequence in $Q$ which takes on infinitely many distinct values has uncountably many limit points in $\beta Q$ and so is not convergent. 
If $A$ is a subset of $\beta Q$ we let $A_{0}=A \cap Q$. We denote by $\bar{A}$ the closure of $A$ in $\beta Q$. Since $Q$ is discrete and dense in $\beta Q$, it is the set of isolated points of $Q$.

Proposition C.3. (a) If $L_{1}, L_{2} \subset Q$ then $\overline{L_{1}} \cap \overline{L_{2}}=\overline{\left(L_{1} \cap L_{2}\right)}$.

(b) If $A \subset \beta Q$ the following are equivalent:

(i) $A$ is a clopen subset of $\beta Q$.

(ii) There exists an open set $U$ such that $A=\bar{U}$.

(iii) There exists $L \subset Q$ such that $A=\bar{L}$.

(iv) $A=\overline{A_{0}}$.

If $L \subset Q$ then $L=(\bar{L})_{0}$.

(c) If $A \subset \beta Q$ is a closed subset, we let $\mathcal{F}_{A}=\{L \subset Q: A \subset \bar{L}\}$. If $A$ is nonempty then $\mathcal{F}_{A}$ is a filter of subsets of $Q$. The collection $\left\{\bar{L}: L \in \mathcal{F}_{A}\right\}$ is the set of clopen neighborhoods of $A$ and so is a base for the neighborhoods of $A$ in $\beta Q . \mathcal{F}_{\emptyset}$ is the power set of $Q$ and the collection $\left\{\bar{L}: L \in \mathcal{F}_{\emptyset}\right\}$ is the set of all clopen subsets of $\beta Q$.

(d) If $E \subset \beta Q \times \beta Q$ is an open equivalence relation then it is a clopen neighborhood of the diagonal $i d_{\beta Q}$. The set of equivalence classes $\{E(p): p \in \beta Q\}$ is a finite clopen partition of $\beta Q$ and $\left\{(E(p))_{0}: p \in \beta Q\right\}$ is a finite partition of $Q$. Conversely, if $\left\{L_{1}, \ldots, L_{k}\right\}$ is a partition of $Q$ then $\left\{\overline{L_{1}}, \ldots, \overline{L_{k}}\right\}$ is a clopen partition of $\beta Q$ with associated clopen equivalence relation $\bigcup_{i} \overline{L_{i}} \times \overline{L_{i}}$. The set of such open equivalence relations forms a neighborhood basis for the diagonal.

Proof: : (a): If $L \subset Q$ then the characteristic function $\chi_{L}: Q \rightarrow\{0,1\}$ extends to a continuous function $\beta \chi_{L}$ on $\beta Q$. Hence, $\left(\beta \chi_{L}\right)^{-1}(1)$ and $\left(\beta \chi_{L}\right)^{-1}(0)$ are disjoint clopen sets containing $L$ and $Q \backslash L$ respectively. Hence, $\bar{L}$ is disjoint from $\overline{Q \backslash L}$, with union $\beta Q$. It follows that $\bar{L}$ is clopen and disjoint subsets of $Q$ have disjoint closures.

For $L_{1}, L_{2}$ the trio $\overline{L_{1} \backslash\left(L_{1} \cap L_{2}\right)}, \overline{\left(L_{1} \cap L_{2}\right)}, \overline{L_{2} \backslash\left(L_{1} \cap L_{2}\right)}$ are pairwise disjoint. Since $\overline{L_{i}}=\overline{L_{i} \backslash\left(L_{1} \cap L_{2}\right)} \cup \overline{\left(L_{1} \cap L_{2}\right)}$ for $i=1,2$ it follows that $\overline{L_{1}} \cap \overline{L_{2}}=\overline{\left(L_{1} \cap L_{2}\right)}$.

(b): (iv) $\Rightarrow$ (iii): Obvious.

(iii) $\Rightarrow$ (i): It was shown above that $L \subset Q$ implies $\bar{L}$ is clopen.

(i) $\Rightarrow$ (ii): Let $U=A$.

(ii) $\Rightarrow$ (iv): If $U$ is open and $\bar{U}=A$ then, since $U \subset A$, we have $U_{0} \subset A_{0} \subset$ $A$. Since $Q$ is dense in $\beta Q$ and $U$ is open, $U_{0}=U \cap Q$ is dense in $U$. Hence, $\overline{U_{0}}=\bar{U}=A$ and so $\overline{A_{0}}=A$.

In general, $L \subset Q$ implies $L \subset \bar{L} \cap Q=(\bar{L})_{0}$. If $L_{1}=(\bar{L})_{0} \backslash L$ then $L_{1} \subset \bar{L}$ and so $\overline{L_{1}} \subset \bar{L}$. But $L_{1}$ is disjoint from $L$ and so $\overline{L_{1}}$ is disjoint from $\bar{L}$. That is, $L_{1}=\emptyset$. 
(c): The family $\mathcal{F}_{A}$ is hereditary upwards and by (a) it is closed under intersection. If $U$ is any closed neighborhood of $A$ and $\hat{U}$ is the closure of the interior of $U$, then by (b) $\hat{U} \subset U$ is a clopen neighborhood of $A$ and equals $\bar{L}$ with $L=(\hat{U})_{0} \in \mathcal{F}_{A}$. The results for $\mathcal{F}_{\emptyset}$ are obvious from (b).

(d): If $E$ is an open equivalence relation on any compact space $X$, then the collection of equivalence classes forms a pairwise disjoint cover of $X$ by open, nonempty subsets. Hence, there are only finitely many equivalence classes $\left\{E_{1}, \ldots, E_{k}\right\}$ and so each is clopen. So $E=\bigcup_{i} E_{i} \times E_{i}$ is clopen in $X \times X$. Then $\left\{\left(E_{1}\right)_{0}, \ldots,\left(E_{k}\right)_{0}\right\}$ is a finite partition of $Q$. Conversely, if $\left\{L_{1}, \ldots, L_{k}\right\}$ is a partition of $Q$ then $\left\{\overline{L_{1}}, \ldots, \overline{L_{k}}\right\}$ is a clopen partition of $\beta Q$ by (a) and (b).

For a compact space, $X$, the neighborhoods of the diagonal form a uniformity and so for any neighborhood $V$ of the diagonal, there exists a symmetric open neighborhood $W$ of the diagonal such that $\bar{W} \circ \bar{W} \subset V$. When $X=\beta Q$ we can choose a finite list $x_{1}, \ldots, x_{k} \in \beta Q$ so that $\left\{W\left(x_{1}\right), \ldots, W\left(x_{k}\right)\right\}$ is a finite open cover. Let $E_{1}=\overline{W\left(x_{1}\right)}$ and $E_{i}=\overline{W\left(x_{i}\right)} \backslash \bigcup_{j<i} \overline{W\left(x_{j}\right)}$. Each of these is clopen by (b). Thus, $\left\{E_{1}, \ldots, E_{k}\right\}$ is a finite clopen partition of $\beta Q$ and $E=\bigcup_{i} E_{i} \times E_{i} \subset V$ because $\bar{W} \circ \bar{W} \subset V$.

We will denote by $\mathcal{E}$ the set of clopen equivalence relations on $\beta Q$. $\mathcal{E}$ is closed under intersection and is directed by (decreasing) inclusion.

If $X$ is a compact space we let $2^{X}$ denote the set of closed subsets of $X$ (including $\emptyset$ ). For a compact Hausdorff space the set of neighborhoods of the diagonal is a uniformity on $X$, in fact, the unique uniformity with the topology of $X$, [34, Corollary 6.30]. For $V$ a neighborhood of the diagonal in $X \times X$ we define $\hat{V} \subset 2^{X} \times 2^{X}$ by $\hat{V}=\{(A, B): B \subset V(A)$, and $A \subset V(B)\}$. The collection of $\hat{V}^{\prime}$ 's generate a uniformity on $2^{X}$ with a compact Hausdorff topology, see e.g. [2, pp. 92-93]. We will restrict our discussion to the case of $X=\beta Q$ so that we need only consider the equivalence relations $E \in \mathcal{E}$ as these generate the neighborhoods of the diagonal. Observe that $B \subset E(A)$ iff $E(B) \subset E(A)$ since $E$ is an equivalence relation. Hence, $\hat{E}=\{(A, B) \in$ $\left.2^{\beta Q} \times 2^{\beta Q}: E(A)=E(B)\right\}$. Since each equivalence class of $E$ is clopen we can choose from it a point of $Q$ and thus obtain a finite set $F^{E} \subset Q$ such that $F^{E}$ meets each equivalence class in a single point. If $A \in 2^{\beta Q}$ then $E(A) \cap F^{E}$ meets the same $E$ equivalence classes as does $A$. Hence, $\left(E(A) \cap F^{E}, A\right) \in \hat{E}$. Since the power set of $F^{E}$ is finite, it follows that the uniformity on $2^{\beta Q}$ is totally bounded. To show that $2^{\beta Q}$ is compact, it suffices to show that the uniformity is complete, see [34, Theorem 6.32]. 
For $\left\{A^{i}\right\}$ a net in $2^{\beta Q}$ we define

$$
\begin{aligned}
\overline{L I M}_{i} A^{i} & =\bigcap \overline{\bigcup_{i} A^{j}}, \\
\underline{L I M}_{i} A^{i} & =\bigcap_{E \in \mathcal{E}} \bigcup_{i} \bigcap_{j>i} E\left(A^{j}\right) .
\end{aligned}
$$

Lemma C.4. Let $\left\{A^{i}: i \in I\right\}$ be a net in $2^{\beta Q}$ and $A \in 2^{\beta Q}$.

(a) $\underline{L I M}_{i} A^{i} \subset \overline{L I M}_{i} A^{i}$.

(b) $A \subset \underline{L I M_{i}} A^{i}$ iff for all $E \in \mathcal{E}$, eventually, $A \subset E\left(A^{i}\right)$, or, equivalently $E(A) \subset E\left(A^{i}\right)$.

(c) $\overline{L I M}_{i} A^{i} \subset A$ iff for all $E \in \mathcal{E}$, eventually, $A^{i} \subset E(A)$, or, equivalently, $E\left(A^{i}\right) \subset E(A)$.

(d) The following are equivalent

(i) $\underline{L I M}_{i} A^{i}=\overline{L I M}_{i} A^{i}$.

(ii) There exists $A \in 2^{\beta Q}$ such that for all $E \in \mathcal{E}$, eventually, $E\left(A^{i}\right)=$ $E(A)$.

(iii) With $A=\overline{L I M}$ for all $E \in \mathcal{E}$, eventually, $E\left(A^{i}\right)=E(A)$.

(iv) For all $E \in \mathcal{E}$, there exists $i \in I$ such that $j_{1}, j_{2}>i$ imply $E\left(A^{j_{1}}\right)=E\left(A^{j_{2}}\right)$.

Proof: (a): Fix $E \in \mathcal{E}$. Clearly,

$$
\bigcup_{i} \bigcap_{j>i} E\left(A^{j}\right) \subset \bigcap_{i} \bigcup_{j>i} E\left(A^{j}\right)=\bigcap_{i} E\left(\bigcup_{j>i} A^{j}\right) \text {. }
$$

Now intersect over $E \in \mathcal{E}$.

$$
\begin{array}{r}
\underline{L I M}_{i} A^{i} \subset \bigcap_{E} \bigcap_{i} E\left(\bigcup_{j>i} A^{j}\right)= \\
\bigcap _ { i } E ( \bigcup _ { j > i } A ^ { j } ) = \bigcap \longdiv { \bigcup _ { j > i } A ^ { j } } = \overline { L I M _ { i } } A ^ { i } .
\end{array}
$$

(b): It is clear that if for every $E \in \mathcal{E}$ there exists $i$ such that $A \subset E\left(A^{j}\right)$ for all $j>i$ then $A \subset \underline{L I M}$. On the other hand, if $A \subset \underline{L I M}$ then for every $E$ $A \subset \bigcup_{i} \bigcap_{j>i} E\left(A^{j}\right)$. Since there are only finitely many $E$ equivalence classes and each is open, this is an increasing union of open sets. It follows that for some $i, A \subset \bigcap_{j>i} E\left(A^{j}\right)$. Thus, $A \subset E\left(A^{j}\right)$ for all $j>i$.

(c): If for each $E \in \mathcal{E}$, there exists $j$ such that $A^{j} \subset E(A)$ for all $j>i$ then since $E(A)$ is closed, $\overline{L I M} \subset E(A)$. Intersecting over $E$ we obtain $\overline{L I M} \subset A$. On the other hand, if $\overline{L I M} \subset A$ and $E \in \mathcal{E}$ then since $E(A)$ is open and $\bigcap_{i} \overline{\bigcup_{j>i} A^{j}}$ is a decreasing intersection of compact sets, it follows that for some $i, \overline{\bigcup_{j>i} A^{j}} \subset E(A)$ and so for $j>i, A^{j} \subset E(A)$. 
The terminal equivalences in (b) and (c) follow from the fact that $B \subset E(A)$ iff $E(B) \subset E(A)$ since $E$ is an equivalence relation.

(d): (i) $\Rightarrow$ (iii): If $A=\overline{L I M}=\underline{L I M}$ then by (b) and (c), for every $E \in \mathcal{E}$, eventually $E\left(A^{i}\right) \subset E(A)$ and eventually $E(A) \subset E\left(A^{i}\right)$.

(iii) $\Rightarrow$ (ii): Obvious.

(ii) $\Rightarrow$ (i): This follows from (a), (b) and (c).

(ii) $\Rightarrow$ (iv): Obvious.

(iv) $\Rightarrow$ (i): If $E \in \mathcal{E}$ and $E\left(A^{j}\right)$ is constant for $j>i$ then

$$
\begin{array}{r}
\overline{L I M} \subset \overline{\bigcup_{j>i} A^{j}} \subset E\left(\bigcup_{j>i} A^{j}\right) \\
=\bigcup_{j>i} E\left(A^{j}\right)=\bigcap_{j>i} E\left(A^{j}\right) \subset \bigcup_{i} E\left(A^{j}\right) .
\end{array}
$$

Intersecting over $E \in \mathcal{E}$ we obtain $\overline{L I M} \subset \underline{L I M}$. The reverse inclusion is always true by (a).

Corollary C.5. (a) A net $\left\{A^{i}: i \in I\right\}$ in $2^{\beta Q}$ is Cauchy iff $\overline{L I M}_{i} A^{i}=$ $\underline{\operatorname{LIM}}_{i} A^{i}$ in which case this common value is the limit. In particular, $2^{\beta Q}$ is complete and so is compact.

(b) If $A \in 2^{\beta Q}$ then $\left\{E(A) \cap F^{E}: E \in \mathcal{E}\right\}$ is a net, indexed by $\mathcal{E}$, consisting of finite subsets of $Q$, which converges to $A$.

Proof: (a): The net is Cauchy iff for every $E \in \mathcal{E}$, there exists $i \in I$ such that $j_{1}, j_{2}>i$ imply $\left(A^{j_{1}}, A^{j_{2}}\right) \in \hat{E}$, i.e. $E\left(A^{j_{1}}\right)=E\left(A^{j_{2}}\right)$. By Lemma C.4 (d) this holds iff $\overline{L I M}=\underline{L I M}$ and in that case with $A$ this common value we have eventually $E\left(A^{i}\right)=E(A)$ for all $E \in \mathcal{E}$. That is, eventually $\left(A^{i}, A\right) \in \hat{E}$. This means that the net converges to $A$.

(b): We observed above that $\left(E(A) \cap F^{E}, A\right) \in \hat{E}$. Thus, the net converges to $A$.

Proposition C.6. Let $\left\{A^{i}: i \in I\right\}$ be a net in $2^{\beta Q}$ and $A \in 2^{\beta Q}$.

(a) If $A=\emptyset$ then $\left\{A^{i}: i \in I\right\}$ converges to $A$ iff eventually $A^{i}=\emptyset$. That is, $\emptyset$ is an isolated point of $2^{\beta Q}$.

(b) Let $F$ be a finite subset of $Q$. If $\left\{A^{i}: i \in I\right\}$ converges to $A$ then eventually $A^{i} \cap F=A \cap F$. If $A=F$ then eventually $A^{i}=F$. That is, $F$ is an isolated point of $2^{\beta Q}$. In general,

$$
A_{0}=\bigcup_{i} \bigcap_{j>i}\left(A^{i}\right)_{0}=\bigcap_{i} \bigcup_{j>i}\left(A^{i}\right)_{0}
$$


(c) $\left\{A^{i}: i \in I\right\}$ converges to $A$ iff for every $L \in \mathcal{F}_{A}$ eventually $L \in \mathcal{F}_{A^{i}}$ and for every $L \notin \mathcal{F}_{A}$ eventually $L \notin \mathcal{F}_{A}^{i}$.

(d) If for every $i \in I, A^{i}=\overline{S^{i}}$ with $S^{i} \subset Q$ then $\left\{A^{i}: i \in I\right\}$ converges to $A$ iff for every $L \in \mathcal{F}_{A}$ eventually $S^{i} \subset L$ and for every $L \notin \mathcal{F}_{A}$ eventually $S^{i} \cap(Q \backslash L) \neq \emptyset$.

Proof: (a): For any $E \in \mathcal{E} E(\emptyset)=\emptyset$. So convergence implies that eventually $E\left(A^{i}\right)=\emptyset$ and this requires $A^{i}=\emptyset$. Conversely, if $A^{i}=\emptyset$ eventually then the net converges to $\emptyset$.

(b): We may assume that $F$ is nonempty and we let $E_{F}=\{\overline{Q \backslash F} \times \overline{Q \backslash F}\} \cup$ $\{(k, k): k \in F\} \in \mathcal{E}$. Convergence implies that eventually $E_{F}\left(A^{i}\right)=E_{F}(A)$. This implies that $A^{i}$ contains the same points of $F$ as does $A$, i.e. $A^{i} \cap F=$ $A \cap F$. Furthermore, if $A=F$ and $E_{F}\left(A^{i}\right)=E_{F}(A)$, then $A^{i} \cap \overline{Q \backslash F}=\emptyset$ and so $A^{i}=F$. In particular, if $k \in A_{0}$ then eventually $k \in A^{i}$ and so $k \in\left(A^{i}\right)_{0}$. If $k \in Q \backslash A_{0}$ then eventually $k \notin\left(A^{i}\right)_{0}$.

(c): For $L \subset Q$ let $E_{L}=\bar{L} \times \bar{L} \cup \overline{Q \backslash L} \times \overline{Q \backslash L}$. Convergence implies that eventually $E_{L}\left(A^{i}\right)=E_{L}(A)$. If $L \in \mathcal{F}_{A}$ then $E_{L}(A)=\bar{L}$ and so $A^{i} \subset \bar{L}$, i.e. $L \in \mathcal{F}_{A^{i}}$. If $L \notin \mathcal{F}_{A}$ then $\overline{Q \backslash L} \subset E_{L}(A)$ and so $A^{i}$ meets $\overline{Q \backslash L}$.

If $A=\emptyset$ then $\emptyset \in \mathcal{F}_{A}$ and the condition implies that eventually $\emptyset \in \mathcal{F}_{A^{i}}$, i.e. eventually $A^{i}=\emptyset$. We now assume that $A$ is nonempty.

Now assume that the condition holds and that the equivalence classes of $E$ are $\overline{L_{1}}, \ldots, \overline{L_{k}}$ with $\left\{L_{1}, \ldots L_{k}\right\}$ a partition of $Q$. Assume that they have been numbered so that for some $1 \leq k_{1} \leq k, E(A)=\bigcup_{n=1}^{k_{1}} \overline{L_{n}}$. Then $\bigcup_{n=1}^{k_{1}} L_{n} \in \mathcal{F}_{A}$ and so there exists $i_{0} \in I$ so that $j>i_{0}$ implies $\bigcup_{n=1}^{k_{1}} L_{n} \in \mathcal{F}_{A^{j}}$. For each $m=1, \ldots, k_{1}, Q \backslash L_{m} \notin \mathcal{F}_{A}$ and so there exists $i_{m}$ such that $j>i_{m}$ implies that $A^{j}$ meets $\overline{L_{m}}$. Choose $i>i_{0}, \ldots, i_{k_{1}}$. If $j>i$ then $E\left(A^{i}\right)=E(A)$. It follows that $\left\{A^{i}: i \in I\right\}$ converges to $A$.

(d): If $A^{i} \subset \bar{L}$ then, intersecting with $Q$ we have $S^{i}=\left(A^{i}\right)_{0} \subset(\bar{L})_{0}=L$. If $A^{i}$ meets $\overline{Q \backslash L}$ then $S^{i}$ meets $Q \backslash L$ since disjoint subsets of $Q$ have disjoint closures in $\beta Q$. In each case, the converse is obvious.

Assume $\phi: \Gamma \times Q \rightarrow Q$ is a monoid action of a countable, discrete, abelian monoid $\Gamma$ on a set $Q$. For each $\gamma \in \Gamma$, we obtain the continuous map $\beta \phi^{\gamma}$ : $\beta Q \rightarrow \beta Q$. Since $\beta \phi^{\gamma_{1}} \circ \beta \phi^{\gamma_{2}}=\beta\left(\phi^{\gamma_{1}} \circ \phi^{\gamma_{2}}\right)=\beta \phi^{\gamma_{1} \gamma_{2}}$, we obtain a continuous monoid action which we denote $\bar{\phi}: \Gamma \times \beta Q \rightarrow \beta Q$.

Apply this to the translation action of $\Gamma$ on itself, i.e. with $\phi=M: \Gamma \times \Gamma \rightarrow$ $\Gamma$ and we obtain the action $\bar{M}: \Gamma \times \beta \Gamma \rightarrow \beta \Gamma$ of $\Gamma$ on the compact space $\beta \Gamma$.

In general, if $\Phi: \Gamma \times X \rightarrow X$ is a continuous monoid action of $\Gamma$ on a compact space $X$ then for each $x \in X$, we obtain the continuous extension $\beta \Phi_{x}: \beta \Gamma \rightarrow X$. This defines a map $\beta \Phi: \beta \Gamma \times X \rightarrow X$. In general, it is 
not continuous, but for each $x \in X,(\beta \Phi)_{x}=\beta \Phi_{x}: \beta \Gamma \rightarrow X$ is continuous and for each $\gamma \in \Gamma,(\beta \Phi)^{\gamma}=\Phi^{\gamma}: X \rightarrow X$ is continuous. That is, $p \rightarrow p x$ is continuous for each $x \in X$ and $x \rightarrow \gamma x$ is continuous for each $\gamma \in \Gamma$.

For $\gamma, \tau \in \Gamma$ and $x \in X$, the equation $(\gamma \tau) x=\gamma(\tau x)$ implies that

$$
\beta \Phi_{x} \circ \beta M^{\gamma}=\Phi^{\gamma} \circ \beta \Phi_{x}
$$

holds on $\Gamma$ and so by continuity on $\beta S$.

Apply this to $\bar{M}: \Gamma \times \beta \Gamma \rightarrow \beta \Gamma$ and we obtain $\beta M: \beta \Gamma \times \beta \Gamma \rightarrow \beta \Gamma$ and the above equation implies that $\beta M$ is an associative multiplication and so gives $\beta \Gamma$ the structure of an Ellis semigroup. Furthermore, if $\Phi: \Gamma \times X \rightarrow X$ is a continuous action of $S$ on a compact space $X$ then $\beta \Phi: \beta \Gamma \times X \rightarrow X$ is an Ellis action.

Observe that if 1 is the identity in $\Gamma$ then it is the identity in $\beta \Gamma$. That is, $p 1=p=1 p$ because the equations are true for $p \in S$ and since $1 \in$ $S$, both $p \mapsto p 1$ and $p \mapsto 1 p$ are continuous. Although $\beta \Gamma$ is usually not abelian, it is true that $p \gamma=\gamma p$ is true for all $p \in \beta \Gamma$ and $\gamma \in \Gamma$. If $h$ : $\Gamma \rightarrow S$ is a homomorphism with $S$ an Ellis semigroup, then $\beta h: \beta \Gamma \rightarrow S$ is a homomorphism. Observe that $\beta h(p q)=\beta h(p) \beta h(q)$ is true for $p, q \in \Gamma$ because $\beta h$ extends the homomorphism $h$. For $p$ fixed in $\Gamma$ continuity implies the equation holds for $q \in \beta \Gamma$ and so with $q$ fixed in $\beta \Gamma$ continuity implies the result for all $p \in \beta \Gamma$. In particular, a homomorphism $h: \Gamma_{1} \rightarrow \Gamma_{2}$ of discrete monoids extends to a continuous homomorphism $\beta h: \beta \Gamma_{1} \rightarrow \beta \Gamma_{2}$.

If $\phi: \Gamma \times X \rightarrow X$ is a continuous action of $\Gamma$ on a compact space $X$, then the homomorphism $\phi^{\#}: \Gamma \rightarrow X^{X}$ extends to a continuous homomorphism of Ellis semigroups $\beta \phi^{\#}: \beta \Gamma \rightarrow X^{X}$. The image is the closure of $\phi^{\#}(\Gamma) \subset X^{X}$, i.e. the enveloping semigroup $E(X, \Gamma)$.

Assume $\phi: \Gamma \times X \rightarrow X$ and $\psi: \Gamma \times Y \rightarrow Y$ are continuous actions of $\Gamma$ on the compact spaces $X$ and $Y$ and that $\pi: X \rightarrow Y$ is a continuous action map. The map is an action map between the Ellis actions because the equation $\pi(p x)=p \pi(x)$ extends from $p \in \Gamma$ to $p \in \beta \Gamma$ by continuity for each fixed $x \in X$.

Recall that $\Gamma_{u}$ is the group of units in $\Gamma$, i.e. the set of $\gamma$ such that $\gamma \tau=1$ for some $\tau \in \Gamma$.

Let $\beta^{*} \Gamma=\beta \Gamma \backslash \Gamma$ and let $\beta^{\prime} \Gamma=\beta \Gamma \backslash \Gamma_{u}$. We call $\Gamma$ a cancelation monoid if for each $\gamma \in S$ the translation maps $M^{\gamma}$ and $M_{\gamma}$ are injective. Of course, if $\Gamma$ is a group then it is a cancelation monoid.

Lemma C.7. Assume that $\Gamma$ is a cancelation monoid.

(a) If $p, q \in \beta \Gamma$, then $p q \in \Gamma$ iff $p, q \in \Gamma$ and $p q \in \Gamma_{u}$ iff $p, q \in \Gamma_{u}$.

$$
\beta \Gamma \cdot \beta^{*} \Gamma \cup \beta^{*} \Gamma \cdot \beta \Gamma \subset \beta^{*} \Gamma, \beta \Gamma \cdot \beta^{\prime} \Gamma \cup \beta^{\prime} \Gamma \cdot \beta \Gamma \subset \beta^{\prime} \Gamma .
$$

(b) The identity 1 is the only idempotent element of $\Gamma$. 
Proof: (a): If $p, q \in \Gamma$ then of course $p q \in \Gamma$. Assume $t=p q \in \Gamma$. Since $z \mapsto z q$ is continuous and $\Gamma$ is discrete the set $A=\{z \in \beta \Gamma: z q=t\}$ is a clopen set and so if it meets $\beta^{*} \Gamma$ then it meets $\Gamma$ in an infinite set. Let $\gamma \in A \cap \Gamma$. If $B=\{w \in \beta \Gamma: \gamma w=t\}$ meets $\beta^{*} S$ then it meets $\Gamma$ in an infinite set. This is impossible since $M^{\gamma}$ is injective on $\Gamma$. It follows that $B \subset \Gamma$ and so $q \in B$ is in $\Gamma$. Hence, $M_{q}$ is injective implies that $A \cap \Gamma$ is not infinite and so $p \in A$ is also in $S$.

Thus, if either $p$ or $q$ is in $\beta^{*} S$ then $p q \in \beta^{*} S$.

If $p q \in \Gamma_{u}$ then, as above, $p, q \in \Gamma$. There exists $\tau$ which is an inverse for $p q$ and so $q \tau$ is an inverse for $p$ and $p \tau$ is an inverse for $q$, because $\Gamma$ is abelian. Hence, $p, q \in \Gamma_{u}$.

(b) If $\gamma \gamma=\gamma=\gamma 1$ then $\gamma=1$ by cancelation.

If $\Gamma$ is a cancelation monoid such that $\gamma \tau=1$ only when $\gamma=\tau=1$, i.e. if $\Gamma_{u}=\{1\}$, then we say that $\Gamma$ is a cancelation monoid without inverses.

Lemma C.8. If $\Gamma$ is a group then $\beta^{*} \Gamma=\beta^{\prime} \Gamma$. If $\Gamma$ is a cancelation monoid without inverses, then $\beta^{\prime} \Gamma$ is the closure in $\beta \Gamma$ of $\Gamma^{\prime}=\Gamma \backslash\{1\}$.

Proof: $\Gamma$ is a group exactly when $\Gamma=\Gamma_{u}$.

Since 1 is an isolated point in $\beta \Gamma, \Gamma^{\prime}$ is dense in $\beta^{\prime} \Gamma=\beta \Gamma \backslash\{1\}$.

Of course, $\mathbb{Z}$ is a group, while $\mathbb{Z}_{+}$and $F I N(\mathbb{N})$ are cancelation monoids without inverses.

Definition C.9. If $\phi: \Gamma \times X \rightarrow X$ is a continuous action of $\Gamma$ on $X$ with $\Gamma$ a cancelation monoid then we define the adherence semigroup

$$
A(X, \Gamma)=\beta \phi^{\#}\left(\beta^{\prime} \Gamma\right) \subset E(X, \Gamma) .
$$

From (C.2) it follows that $A(X, \Gamma)$ is an ideal in $E(X, \Gamma)$.

For a cascade, $(X, T)$ with $\Gamma$ the group $\mathbb{Z}, A(X, T)$ is the set of limit points as $|n| \rightarrow \infty$ of the bi-infinite sequence $\left\{T^{n}\right\}$ in $E(X, T)$. If $\Gamma$ is a cancelation monoid without inverses then $A(X, \Gamma)$ is the closure in $E(X, \Gamma)$ of $\left\{\phi^{\gamma}: \gamma \neq 1\right\}$.

Recall that for a system $(X, \Gamma)$ a point $x \in X$ is called recurrent when $x \in \overline{\Gamma^{\prime} \cdot x}$.

Proposition C.10. Assume that $\Gamma$ is a cancelation monoid and that $\Gamma_{u}$ contains no element of finite order other than 1, e.g. a cancelation monoid without inverses. For a system $(X, \Gamma)$ and a point $x \in X$ the following are equivalent.

(i) The point $x$ is recurrent.

(ii) There exists $p \in \beta^{\prime} \Gamma$ such that $p x=x$.

(iii) There exists an idempotent $p \in \beta^{*} \Gamma$ such that $p x=x$. 
(iv) There exists $p \in A(X, \Gamma)$ such that $p x=x$.

(v) There exists an idempotent $p \in A(X, \Gamma)$ such that $p x=x$.

Proof: Let $\phi: \Gamma \times X \rightarrow X$ be the action.

(ii) $\Leftrightarrow$ (iv): For $p \in \beta \Gamma, p x=\phi^{\#}(p) x$.

(ii) $\Leftrightarrow$ (iii), (iv) $\Leftrightarrow(\mathrm{v})$ : The sets $\left\{p \in \beta^{\prime} \Gamma: p x=x\right\}$ and $\{p \in A(X, \Gamma)$ : $p x=x\}$ are closed sub-semigroups. If either is non-empty then it contains idempotents by Lemma B.1. Also, there is no idempotent in $\beta^{\prime} \Gamma \backslash \beta^{*} \Gamma \subset \Gamma^{\prime}$.

(ii) $\Rightarrow$ (i): $\beta^{\prime} \Gamma \cdot x \subset \overline{\Gamma^{\prime}} \cdot x$.

(i) $\Rightarrow$ (ii): If $x$ is recurrent then there exists a net $\left\{\gamma^{i} \in \Gamma^{\prime}\right\}$ such that $\gamma^{i} x \rightarrow x$. If $q$ is a limit point of $\left\{g^{i}\right\}$ in $\beta \Gamma$ then $q x=x$. If $q \in \beta^{\prime} \Gamma$ then let $p=q$ to obtain (ii). Otherwise, $q \in \Gamma_{u}$. Since 1 is an isolated point, $q \neq 1$. The sequence of powers $\left\{q^{n}: n \in \mathbb{N}\right\}$ are all distinct since no element of $\Gamma_{u} \backslash\{1\}$ has finite order. If $p$ is a limit point of the sequence in $\beta \Gamma$ then $p \in \beta^{*} \Gamma \subset \beta^{\prime} \Gamma$ since $\Gamma$ is discrete. Since $q^{n} x=x$ for all $n, p x=x$.

\section{REFERENCES}

1. Ethan Akin, The general topology of dynamical systems, Graduate Studies in Mathematics, 1, American Mathematical Society, Providence, RI, 1993.

2. Ethan Akin, Recurrence in topological dynamical systems: Furstenberg families and Ellis actions, Plenum Press, New York, 1997.

3. Ethan Akin, Lectures on Cantor and Mycielski sets for dynamical systems, Contemporary Mathematics, 356, (2004), 21-79.

4. Ethan Akin, Joseph Auslander and Kenneth Berg, When is a transitive map chaotic, Convergence in Ergodic Theory and Probability, Walter de Gruyter \& Co. 1996, 25-40.

5. Ethan Akin, Joseph Auslander and Kenneth Berg, Almost equicontinuity and the enveloping semigroup, Topological dynamics and applications, Contemporary Mathematics 215, a volume in honor of R. Ellis, 1998, 75-81.

6. Ethan Akin, Joseph Auslander and Eli Glasner, The topological dynamics of Ellis actions, Mem. Amer. Math. Soc. 195 (2008), no. 913.

7. Akin, Ethan Akin and Eli Glasner, Is there a symmetric version of Hindman's theorem?, J. Combin. Theory Ser. A 142 (2016), 29-43.

8. Ethan Akin and Sergii Kolyada, Li-Yorke sensitivity, Nonlinearity, 16, (2003), 14211433.

9. J. Auslander Minimal flows and their extensions North Holland, Amsterdam, 1988.

10. Joseph Auslander and James A. Yorke, Interval maps, factors of maps and chaos, Tohoku Math. Journal 32, (1980), 177-188.

11. Tomasz Downarowicz, Weakly almost periodic flows and hidden eigenvalues, Contemp. Math., 215 (1998), 101-120.

12. W. F. Eberlein, Abstract ergodic theorems and weak almost periodic functions, Trans. Amer. Math. Soc. 67, (1949), 217-240.

13. R. Ellis Lectures on topological dynamics W. A. Benjamin, New York, 1969. 
14. Robert Ellis and Mahesh Nerurkar, Weakly almost periodic flows, Trans. Amer. Math. Soc. 313, (1989), 103-119.

15. H. Furstenberg, Recurrence in ergodic theory and combinatorial number theory, Princeton U. Press, Princeton, 1981.

16. Hillel Furstenberg, and Yitzhak Katznelson, An ergodic Szemerédi theorem for IPsystems and combinatorial theory, J. Analyse Math. 45 (1985), 117-168.

17. Hillel Furstenberg and Benjamin Weiss, Topological dynamics and combinatorial number theory, J. Analyse Math. 34 (1978), 61-85 (1979).

18. Eli Glasner, Ergodic theory via joinings, Math. Surveys and Monographs, AMS, 101, 2003.

19. Eli Glasner, On tame dynamical systems, Colloq. Math. 105 (2006), 283-295.

20. Eli Glasner and David Maon, Rigidity in topological dynamics, Ergod. Th. Dynam. Sys. 9, (1989), 309-320.

21. Eli Glasner and Michael Megrelishvili, Linear representations of hereditarily nonsensitive dynamical systems, Colloq. Math., 104 (2006), no. 2, 223-283.

22. Eli Glasner and Michael Megrelishvili, Eventual nonsensitivity and tame dynamical systems, arXiv:1405.2588.

23. E. Glasner, M. Megrelishvili and V. V. Uspenskij, On metrizable enveloping semigroups, Isr. J. of Math., 164 (2008), 317-332.

24. Eli Glasner and Benjamin Weiss, Sensitive dependence on initial conditions, Nonlinearity 6 (1993), 1067-1075.

25. Eli Glasner and Benjamin Weiss, Locally equicontinuous dynamical systems, Colloquium Mathematicum, part 2, 84/85 (2000), 345-361.

26. Eli Glasner and Benjamin Weiss, Topological groups with Rokhlin properties, Colloq. Math., 110, (2008), no. 1, 51-80.

27. Eli Glasner and Benjamin Weiss, On the interplay between measurable and topological dynamics, Handbook of dynamical systems. Vol. 1B, pp. 597-648, Elsevier B. V., Amsterdam, 2006.

28. Eli Glasner and Xiangdong Ye, Local entropy theory, Ergodic Theory Dynam. Systems, 29, (2009), no. 2, 321-356.

29. T. N. T. Goodman, Topological sequence entropy, Proc. London Math. Soc., 29 (1974), $331-350$.

30. W. H. Gottschalk and G. A. Hedlund, Topological Dynamics, AMS Colloquium Publications, Vol. 36, 1955.

31. Wen Huang and Xiangdong Ye, Homeomorphisms with the whole compacta being scrambled sets, Ergodic Theory Dynam. Systems, 21, (2001), no. 1, 77-91.

32. Yitzhak Katznelson and Benjamin Weiss, When all points are recurrent/generic, Ergodic theory and dynamical systems, I (College Park, Md., 1979-80), 195-210, Progr. Math., 10, Birkhäuser, Boston, 1981.

33. Alexander Kechris and Christian Rosendal, Turbulence, amalgamation, and generic automorphisms of homogeneous structures, Proc. Lond. Math. Soc., (3), 94, (2007), no. 2, 302-350.

34. John Kelley, General Topology, D.Van Nostrand Company, 1955.

35. David Kerr and Hanfeng Li, Independence in topological and $C^{*}$-dynamics, Math. Ann. 338 (2007), 869-926.

36. T. Li and J. Yorke, Period 3 implies chaos, Amer. Math. Monthly, 82 (1975), 985-992.

37. Wolfgang Ruppert, On weakly almost periodic sets, Semigroup Forum, 32 (1985) 267281. 
38. S. A. Shapovalov, A new solution of one Birkhoff problem, J. Dynam. Control Systems, 6 (2000), no. 3, 331-339. 


\section{INDEX}

\section{Index of terms}

action map (homomorphism) 11

adherence semigroup 15, 133

almost periodic (AP) 2

asymptotic pair 116

Banach density (upper) 58

Bernoulli shift, $S 3$

Birkhoff center 15

center periodic $(\mathrm{CP}) 17$

center trivial (CT) 17

coalescence 22

E-coalescence 23

cofinal constant 80

completely scrambled 116

cross-section 77

Ellis semigroup 123

enveloping semigroup 11

equicontinuous 21

equicontinuity point 21

almost equicontinuous (AE) 21

hereditarily almost equicontinuous

(HAE) 21

locally equicontinuous (LE) 22

uniformly equicontinuous 21

expansive 21

expansion of integers in $I P(k) 56$

expansion of length $r 56$

length vector 59

truncation $(\tilde{t}) 56$

extension 56

residual $(t-\tilde{t}) 56$

external element 78

f-contains 37

Gamow transformations 106

height (for dynamical systems) 16

height* (for CT-WAP dynamical systems) 30

height (for labels) 111, 115

height* (for labels) 115

hereditary collection (of subsets of $\mathbb{N}$ ) 88

idempotent 11

independent set (for a dynamical system) 100

independent set (for a label) 100

labels 36 bounded 37

disjoint 89

finitary 81

finite type 37

flat 102

null 100

positive 38

recurrent 46

semi-simple 79

simple 79

size bounded 37

strong finite type 79

strongly recurrent 47

strongly recurrent set 47

tame 100

WAP 78

$L$-determined 27

Li-Yorke pair 116

minimal trivial $(\operatorname{minCT}) 31$

non-wandering 7,15

nucleus (of a labeled subshift) 71

null (dynamical system) 100

orbit closed 27

proximal (pair) 116

proximal (system) 116

recurrent point 12

rigid 23

weakly rigid 23,20

uniformly rigid 23

roof (of a label) 37

scrambled set 116

size of $\mathbf{m} 36$

strongly recurrent set 47

Stone-Cech compactification 126

subshift 3

tame 100

topological transitivity 6

chain transitivity 6

transitive point 6

translation finite (TF) 98

wandering (open set) 7,15

weakly almost periodic (WAP) 2, 12

weak mixing 6,17 


\section{Index of symbols}

$[a \pm b],[ \pm b] 55$

$A(e) 28$

$A[\mathcal{M}], A_{+}[\mathcal{M}] 59$

$\mathcal{A}(\Phi)(F I N(\mathbb{N})$ adherence semigroup) 51

$A(X, T)$ (adherence semigroup) 6

$A_{+}(X, T) 116$

ASYMP 116

$\mathcal{B}_{N}$ (basic labels) 37

$\beta_{b} \mathbb{Z} 68$

CT-WAP 28

$D, D_{+} 69,71$

$\bar{D}, \bar{D}_{+} 85$

$\hat{D}, \hat{D}_{+} 86$

$d\left(\mathcal{M}_{1}, \mathcal{M}_{2}\right)$ (label ultrametric) 39

$e=\overline{0} 18$

$\mathcal{E}$ (set of clopen equivalence relations) 128

$\mathcal{E}_{0}(\Theta(\mathcal{M})) 78$

$\varepsilon_{*}(\Theta(\mathcal{M})) 78$

$\mathcal{E}(\Phi)(F I N(\mathbb{N})$ enveloping semigroup) 51

$\mathcal{E}(\mathcal{L} \mathcal{A B}) 51$

$\hat{\mathcal{E}}(\mathcal{L} \mathcal{A B}) 69$

$E(X, T)$ (enveloping semigroup) 11

$E_{b}(X(\mathcal{M}), S) 70$

$F(\mathbf{m}) 47$

$F I N(L) 106$

$F I N(\mathbb{N}) 36$

$\beta F I N(\mathbb{N}) 44$

$\beta^{*} F I N(\mathbb{N}) 44$

$\beta^{\prime} F I N(\mathbb{N}) 44$

$\mathcal{F}(\mathcal{M}, L) 104$

$\Phi(Y), \Phi_{+}(Y)$ (the preimage operation)

$$
71
$$

$\Gamma^{\prime} 7$

$\Gamma_{u}$ (group of units) 8

INC 40

$I P(k), I P_{+}(k) 56$

$I S O(\mathcal{N}) 46$

$k(n)$ (expanding function) 55

$\ell(n)$ (support map) 59

$\mathcal{L A \mathcal { B }}$ (space of labels) 39

$\mathcal{L} \mathcal{A} \mathcal{B}_{+}$(space of labels) 108

$\mathcal{L A B}(L)$ (space of labels) 106

LIMINF 40

LIMSUP 40

$L I M 41$ $m_{b} 68$

$\mathcal{M}, \mathcal{N}$ labels 36

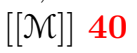

$\max \mathcal{M} 38$

$\mathcal{M} \oplus \mathcal{N} 53$

$\mathbf{m}, \mathbf{r} \mathbb{N}$-vectors 36

$|\mathbf{m}|$ (norm of a label) 36

$n_{*}(t), n^{*}(t) 55,56$

$\operatorname{Nuc}(X(\mathcal{M}))$ (nucleus) 71

$O_{T}$ (relations) 6

$R_{T} 6$

$R_{T}^{*} 6$

$\alpha_{T} 6$

$\omega_{T} 6$

$\mathcal{O}_{T}(x)$ or $\mathcal{O}(x), \overline{\mathcal{O}}_{T}(x)$ (orbit and orbit closure of $x) 12$

$p_{\mathbf{r}} 85$,

$P_{\mathrm{r}} 43$

$\mathcal{P}_{f}(L) 37$

PROX 116

RECUR 54

$\mathbf{r}(t)$ (length vector) 59

$\rho(\mathcal{M})$ (roof function) 37

$S$ (shift) 3

$\langle S\rangle$ (label generated by $S$ ) 37

supp $\mathbf{m} 36$

supp $x 36$

Supp M 38

SYM 75

$\Theta(\mathcal{M})(F I N(\mathbb{N})$ orbit closure $) 45$

$\Theta^{\prime}(\mathcal{N}) 45$

$\Theta(\Psi) 71$

$U$ (minimal idempotent) 52

$\hat{U} 69$

$V_{d, \epsilon} 32$

$x[\mathcal{M}], x_{+}[\mathcal{M}] 63$

$X(A) 62$

$X(\mathcal{M}), X_{+}(\mathcal{M}) 63$

$(X, T)$ (cascade dynamical system) 3

$(X, \Gamma)$ (semigroup dynamical system) 7

$X(\Psi), X_{+}(\Psi) 71$

$(X, S)$ (subshift) 3

$\chi(A) 36$

$\xi_{A}, \xi_{\mathcal{M}} 65$

$X(\mathcal{P} A) 62$

$\mathbb{Z}, \mathbb{Z}_{+}, \mathbb{Z}_{+\infty} 36$

ZER 75

set operators: 16, 108 
$z_{C A N} 16$

$z_{L A B} 108,110$

$z_{L I M} 16$

$z_{N W} 16$

$z_{\mathcal{M}}^{*} 112$ 
Mathematics Department, The City College, 137 Street and Convent Avenue, New York City, NY 10031, USA

E-mail address: ethanakin@earthlink.net

Department of Mathematics, Tel-Aviv University, Ramat Aviv, Israel

E-mail address: glasner@math.tau.ac.il 